

\section{Nucleation and dynamics of bubbles/droplets in multicomponent liquids}

Binglin Zeng 
Thesis committee members:

Prof. dr. Jennifer L. Herek (chairman)

Prof. dr. ner. nat. Detlef Lohse (promoter)

Prof. dr. ir. Harold J. W. Zandvliet (promoter)

Prof. dr. Yuliang Wang (co-promoter)

Dr. Stefan Kooij

Prof. dr. Devaraj van der Meer

Prof. dr. Sebastien Michelin

Prof. dr. Michel Orrit

Prof. dr. Albert Polman
University of Twente University of Twente University of Twente Beihang University University of Twente University of Twente LadHyX-Ecole Polytechnique Leiden University University of Amsterdam

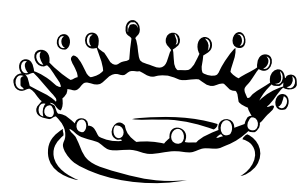

Physics of Fluids
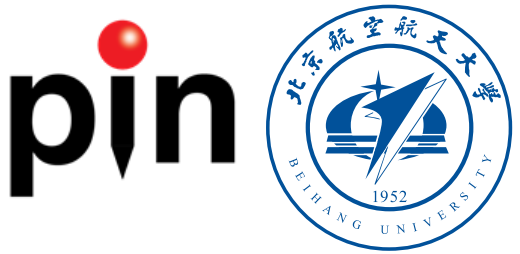

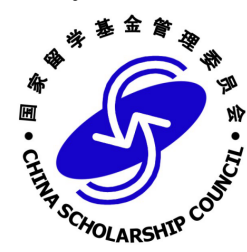

The work in this thesis was carried out at the Physics of Fluids group \& Physics of Interfaces and Nanomaterials group of the Faculty of Science and Technology of the University of Twente, and School of Mechanical Engineering and Automation of Beihang University. This thesis was financially supported by the China Scholarship Council (CSC).

Front cover: Optical image of a bouncing bubble near the gold nanoparticle decorated substrate in acetone water binary liquid under plasmonic resonance.

Publisher:

Binglin Zeng, Physics of Fluids, University of Twente, P.O. Box 217, 7500 AE Enschede, The Netherlands https://pof.tnw.utwente.nl/

Copyright (C) 2021. All rights reserved. No part of this work may be reproduced or transmitted for commercial purposes, in any form or by any means, electronic or mechanical, including photocopying and recording, or by any information storage or retrieval system, except as expressly permitted by the publisher.

ISBN: 978-90-365-5237-0

DOI: $10.3990 / 1.9789036552370$ 


\section{NuCleation AND DYNAMiCS OF BUbBLES/DROPLETS IN MULTICOMPONENT LIQUIDS}

\section{DISSERTATION}

to obtain

the degree of doctor at the University of Twente, on the authority of the rector magnificus,

Prof. dr. ir. A. Veldkamp,

on account of the decision of the graduation committee,

to be publicly defended

on Friday the $24^{\text {th }}$ of September 2021 at 16:45

by

Binglin Zeng

Born on the day of birth $30^{\text {th }}$ of September 1992

in Hunan, China 
This dissertation has been approved by the promoters:

Prof. dr. ner. nat. Detlef Lohse

Prof. dr. ir. Harold J.W.Zandvliet

and the co-promotor:

Prof. dr. Yuliang Wang 


\section{Contents}

1 Introduction 1

1.1 General . . . . . . . . . . . . . . . . . . . . . 1

1.2 Plasmonic bubbles ........................ . . 2

1.3 Micro/nanodroplets from the Ouzo effect . . . . . . . . . . 4

1.4 A guide through the thesis . . . . . . . . . . . . . 5

2 Giant plasmonic bubbles nucleation under different ambient pressures 9

$2.1 \quad$ Introduction $\ldots \ldots \ldots \ldots \ldots \ldots \ldots \ldots \ldots$

2.2 Experimental system $\ldots \ldots \ldots \ldots \ldots$

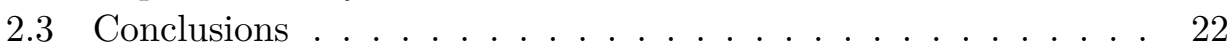

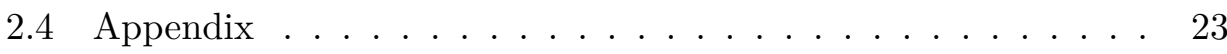

3 Plasmonic bubble nucleation in binary liquids 25

3.1 Introduction . . . . . . . . . . . . . . . . . . . . 27

3.2 Methods and Materials . . . . . . . . . . . . . . . . . . . 28

3.3 Results and discussion $\ldots \ldots \ldots \ldots$

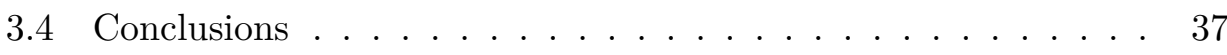

3.5 Appendix $\ldots \ldots \ldots \ldots \ldots \ldots$

4 Periodic bouncing of a plasmonic bubble in a binary liquid by competing solutal and thermal Marangoni forces 41

4.1 Introduction . . . . . . . . . . . . . . . . . . . . . . 43

4.2 Experimental setup and findings $\ldots \ldots \ldots \ldots . \ldots . \ldots . \ldots 44$

4.3 Interpretation of the findings $\ldots \ldots \ldots \ldots . \ldots . \ldots$

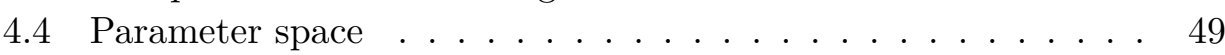

4.5 Theoretical model $\ldots \ldots \ldots \ldots$. . . . . . . . . . . . 50

4.6 Conclusions $\ldots \ldots \ldots \ldots \ldots \ldots \ldots$ 
$4.7 \quad$ Appendix $\ldots \ldots \ldots \ldots \ldots \ldots \ldots$

$5 \quad$ Plasmonic microbubble dynamics in binary liquids 63

5.1 Introduction . . . . . . . . . . . . . . . . . . 65

5.2 Methods and Materials $\ldots \ldots \ldots \ldots \ldots 6$

5.3 Results and discussion $\ldots \ldots \ldots$. . . . . . . . . . 66

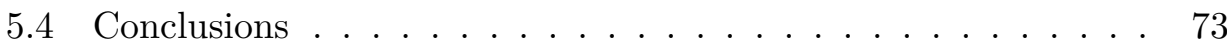

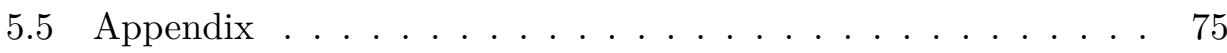

6 Solvent exchange in a Hele-Shaw cell: universality of surface $\begin{array}{ll}\text { nanodroplet nucleation } & 81\end{array}$

$6.1 \quad$ Introduction $\ldots \ldots \ldots \ldots \ldots \ldots \ldots$. . . . . . . . . . . . . . . 83

6.2 Experiment $\ldots \ldots \ldots \ldots \ldots \ldots \ldots$

6.3 Results and Discussion . . . . . . . . . . . . . . . . . . . . . 87

6.4 Conclusions $\ldots \ldots \ldots \ldots \ldots$. . . . . . . . . . . . . . . 92

7 Formation of polystyrene microlenses via transient droplets $\begin{array}{ll}\text { from the ouzo effect for enhanced optical imaging } & 95\end{array}$ 7.1 Introduction $\ldots \ldots \ldots \ldots \ldots \ldots \ldots \ldots$

7.2 Experimental section $\quad \ldots \ldots \ldots \ldots \ldots$

7.3 Results and discussion $\ldots \ldots \ldots \ldots 10 \ldots$

7.4 Conclusions . . . . . . . . . . . . . . . . . 115

8 Sequential evaporation induced formation of polymeric sur$\begin{array}{ll}\text { face microdents via ouzo effect } & 117\end{array}$

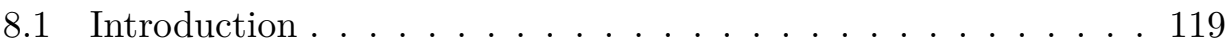

$8.2 \quad$ Experimental section

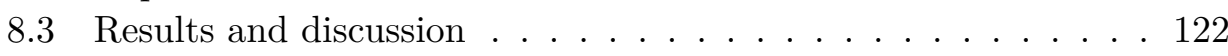

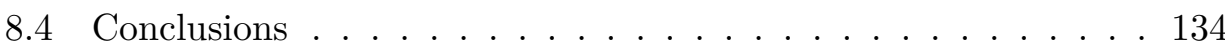

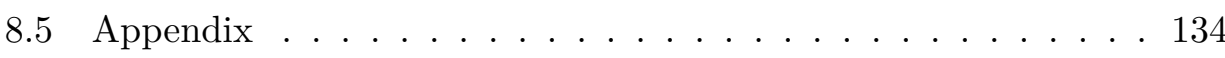

$\begin{array}{lll}9 & \text { Conclusions and outlook } & 137\end{array}$

$\begin{array}{ll}\text { References } & 141\end{array}$

$\begin{array}{ll}\text { Summary } & 165\end{array}$

\begin{tabular}{ll}
\hline Summary (Dutch) & 167
\end{tabular}

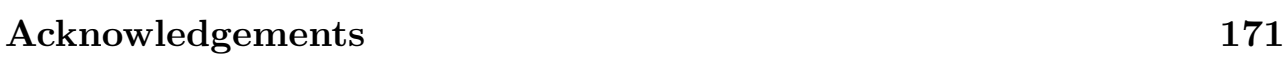


Scientific output 


\section{Chapter 1}

\section{Introduction}

\subsection{General}

Bubbles and droplets are ubiquitous on our planet. They are involved in many natural phenomena. For instance, water vapour in the air turns into tiny droplets at low temperatures, leading to the formation of clouds, fog and rain [1]. Gas is transferred into water and forms microbubbles during wave breaking caused by the strong wind [2]. In addition, bubbles/droplets are present in rhyolites [3], volcanic explosion [4], microorganism fermentation [5], and among others (see examples in Figs. 1.1(a)-(c)). Bubbles/droplets are essential in industrial processes as well: Rocket propulsion occurs due to the combustion of fuel droplets at the microscale; Spray disinfection via microdroplets; Pores in bread caused by carbon dioxide bubbles; ink-jet printing; carbonated beverages with bubbles, and many others [6-10] (see examples in Figs. 1.1(d)-(f)). Nevertheless, the formation of bubbles and droplets can be detrimental as well. During pandemic spreading, microdroplets work as the medium to transmit viruses or bacteria [11]. When manufacturing integrated circuits by immersion lithography, tiny bubbles or droplets in the liquid film can scatter the light and result in defects [12]. Moreover, bubbles and droplets can promote the corrosion of metals, causing damage of turbine blade, bearing, propellers, etc [13].

In general, in practical situations, the formation of bubbles and droplets not only occurs in pure liquids, but also in binary or ternary liquids. In multicomponent liquids, diverse forces and effects play a role [14], such as thermophoresis [15], body forces originated from a density gradient. For the bubbles/droplets at the micro scale, solutal Marangoni forces become relevant 

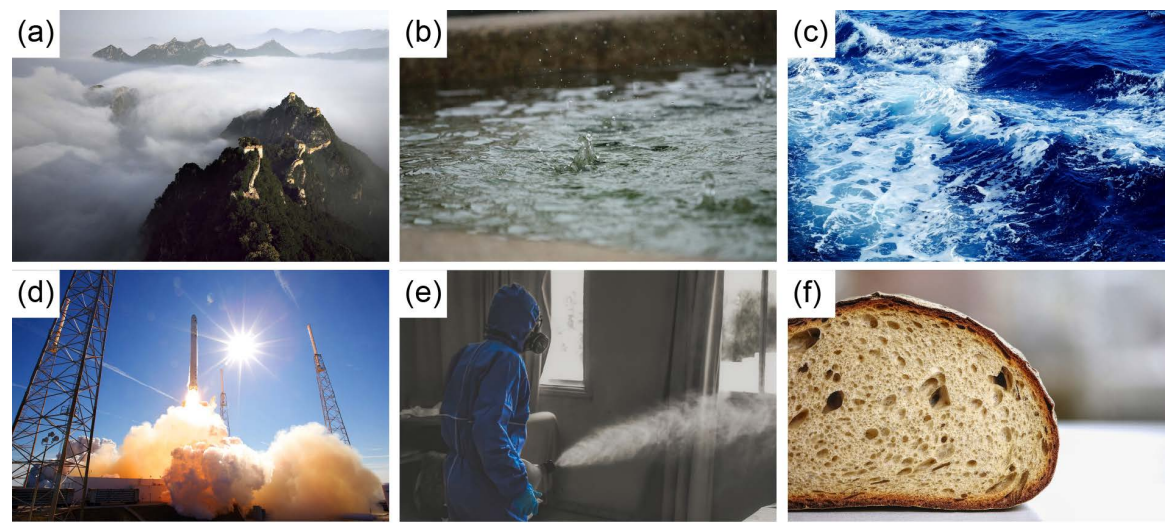

Figure 1.1: Examples of bubbles/droplets in nature and industries. (a) Clouds and fog; (b) Rain; (c) Waves in the sea; (d) Fuel combustion in a rocket engine; (e) Spray disinfection; (f) Pores in the bread. (Source for images: Pixabay website)

as well [16]. Moreover, the behaviour of bubbles and droplets becomes even more complex if phase transitions occur in such systems, e.g., evaporation [17, 18] or the nucleation of a new phase, such as in the Ouzo effect [19, 20]. Over the past several years, bubbles/droplets in multicomponent systems received increasing attention due to their relevance for various applications and the occurrence of numerous counterintuitive phenomena. This thesis is focused on bubbles induced by plasmonic excitation and droplets generated through the Ouzo effect. We will present a detailed study of the dynamics of their nucleation and growth in multicomponent systems.

\subsection{Plasmonic bubbles}

When illuminated by a laser with a specific resonance wavelength, metal nanoparticles can exhibit so-called surface plasmon. In this process, the energy carried by the photons is transferred to collective excitations of free electrons resulting in the production of vast amounts of heat. This enormous heat can vaporize the surrounding liquid when metal particles are immersed in a liquid, leading to the nucleation and growth of plasmonic bubbles. Figs. 1.2 (a) and (b) show the schematic diagrams of a bulk vapor nanobubble generated around a gold nanoparticle (GNP) and a surface plasmonic microbubble on a GNP decorated surface, respectively.

These plasmonic bubbles are of great importance for numerous applications, 

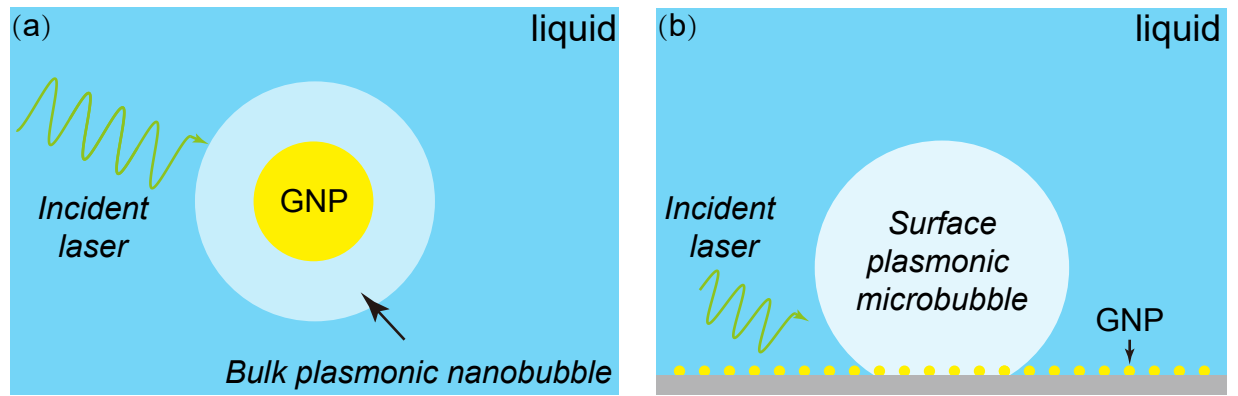

Figure 1.2: Schematic diagrams of a bulk vapor nanobubble generated around a gold nanoparticle (GNP) (a) and a surface plasmonic microbubble on a GNP decorated surface (b) under laser irradiation in liquid.

including catalytic reactions [21], solvothermal chemistry [22], solar harvesting [23 26], cancer therapy and diagnosis [27]. For instance, in a recent study plasmonic particles were injected into target positions of animals with tumors. Under laser irradiation of the particles, tumors in the animals can be killed due to the explosive growth of plasmonic bubbles [27]. In order to properly utilize these bubbles in relevant technologies, it is essential to obtain control over the formation and dynamics of bubbles.

Owing to fundamental interest and technological relevance, these bubbles have been widely studied both theoretically and experimentally. These studies were mainly in water, including modeling of heat transfer from nanoparticles to the surrounding liquid [28] and corresponding phase transition processes [29], characterization of the life phase of the plasmonic microbubble [30], investigation of the control parameters on bubble nucleation, growth and shrinkage [31-33], and many others [34-36]. Results revealed that the life of a surface plasmonic microbubble in water includes 4 phases (Fig. 1.3). Many factors such as laser power, nanoparticle size and arrangements, liquid type and gas saturation play a role in governing the growth and nucleation dynamics of these bubbles.

The plasmonic bubble formation and dynamics have not yet been extensively studied in complex liquids, which are more frequently encountered in practice. Complex liquids, such as binary or ternary liquids can introduce more physicochemical properties. It is essential to understand the nucleation mechanism and growth dynamics of plasmonic bubbles in these multiple component liquids. 


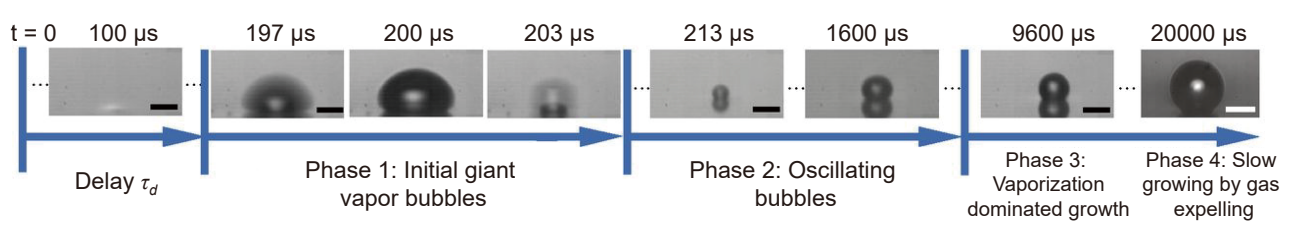

Figure 1.3: The evolution of the plasmonic bubbles in water under a continuous laser irradiation. (Scale bar: $25 \mu \mathrm{m})[30]$.

\subsection{Micro/nanodroplets from the Ouzo effect}

Droplets at the micro/nanoscale on solid substrates are essential for many applications, like analysis for single biomolecular, miniaturized reactors for drug production, super focusing, and templates for micro-manufacture, and among others [37 41].
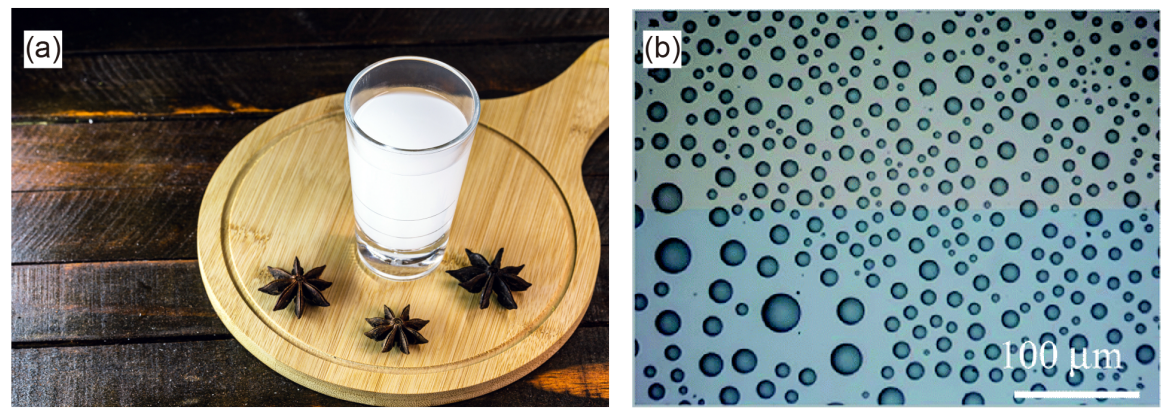

Figure 1.4: (a) Ouzo drink; (Source for this image: Shutterstock website) (b) microdroplets nucleation on substrate based on the Ouzo effect [42].

Over the past decades, many techniques have been developed for the mass production of micro/nanodroplets in controlled manners, like micro-printing, emulsion direct adsorption, trapping by microcavities, and others 10]. Recently, the Ouzo effect has been considered as a simple and generic way to produce droplets in bulk or on substrates. It originates from an anise-flavoured Greek drink, Ouzo, a clear liquid consisting of ethanol, water, and anise oil. When water is added, ouzo turns into a milky colour (Fig. 1.4 (a)). This is because that the anise oil is soluble in ethanol, but not in water. The added water causes the nucleation of anise oil as micro/nanodroplets, which scatter the light. This phenomenon is called "Ouzo effect". It is an attractive approach to form massive micro/nanodroplets without using any surfactant or 
dispersing agents $[43]$.

For the approaches of droplet production through the Ouzo effect, not only the composition in the initial oil-rich solution is important, but also the mixing dynamics of water plays a critical role [20,44,45]. Therefore, compared to the way of directly adding water into an oil-rich solution, the process of solvent exchange is more controllable to trigger the Ouzo effect. In the solvent exchange process, a good solvent is replaced by a poor solvent of oil in a microchannel, resulting in oil micro/nanodroplet nucleation and subsequent growth on a substrate [46] (see Fig. 1.4 (b)).

In recent years, the effects of flow conditions [43], wettability of structures [47], and gravity [48] on the droplet nucleation from the solvent exchange have been studied. Results reveal that the gravity effect can induce substantial convection during the solvent exchange process, leading to considerable inhomogeneities in droplet size [49]. To better apply the Ouzo effect in relevant applications, it is essential to investigate how the droplet size depends on control parameters and to improve the homogeneities of the nucleated droplets.

\subsection{A guide through the thesis}

As shown in Fig. 1.5, this thesis covers two subjects. In the first part, we study the nucleation and growth dynamics of the plasmonic microbubbles under different ambient pressures and in binary liquids. In the second part, we study the microdroplet nucleation through the Ouzo effect in a Hele-Shaw cell, and propose the fabrication methods of microlenses and microindents based on Ouzo effect. Here we give a brief introduction to the topics covered in this thesis. 


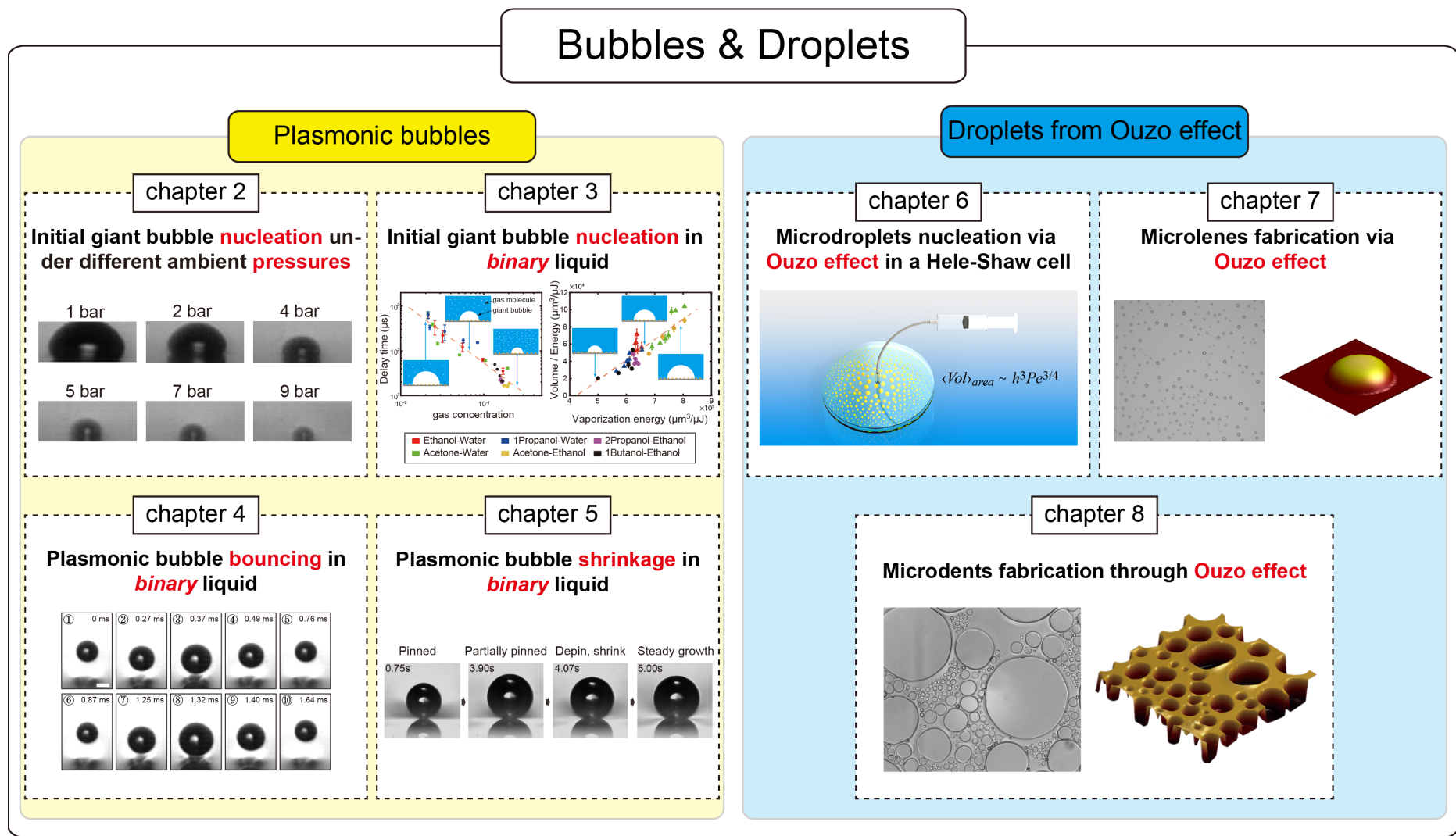

Figure 1.5: The diagram illustrating the outline of this thesis. 


\subsubsection{Part I: Plasmonic bubbles}

In Chapter 2, we investigate the nucleation of the initial giant plasmonic bubbles in water under different ambient pressures on a short time scale of $\mu \mathrm{s}$. The ambient pressure is varied from 1 to 9 bar. We will show how the delay time $\tau_{d}$ of bubble nucleation after turning on the laser and the light-vapour conversion efficiency depend on ambient pressures and the other relevant parameters.

In Chapter 3, we further discuss the nucleation of the initial giant plasmonic bubbles for six different binary liquids (timescale of $\mu \mathrm{s}$ ). The key liquid parameters, which dominate the nucleation of the initial giant bubbles and bubble volume will be investigated.

In Chapter 4 , we study plasmonic bubble behaviour in ethanol-water binary liquids in the ms range. In this study, an interesting bouncing behaviour of the bubble is observed for ethanol concentration $66 \mathrm{wt} \% \leq f_{e} \leq 82 \mathrm{wt} \%$. We reveal the mechanism of this bouncing behaviour, and study the dependence of the bouncing frequency on the control parameters, namely the ethanol fraction and the laser power for the plasmonic heating. Besides, a bullet model is put forward to investigate the bouncing frequencies.

In Chapter 5, we study the dynamics of plasmonic bubbles in ethanol water binary liquid in the seconds timescale. We find that plasmonic bubbles exhibit a sudden shrinkage for ethanol concentrations of $67.5 \mathrm{wt} \% \lesssim f_{e} \lesssim 80 \mathrm{wt} \%$. The mechanism of this sudden shrinkage will be revealed. In addition, we will show how the delay time before bubble shrinkage depends on the laser spot size, laser power and ethanol concentration.

\subsubsection{Part II: Microdroplets and Ouzo effect}

In Chapter 6, we study the Ouzo effect (solvent exchange) process in a wellcontrolled Hele-Shaw geometry with negligible gravitational effects. The nucleated droplets in this study are more homogenous, and the mean droplet volume per area $\langle V o l\rangle_{\text {area }}$ strongly depends on the local Peclet number, Pe, following a universal scaling law. Moreover, the probability distribution function of the droplet volume strongly depends on the local $P e$ as well, regardless of the flow rates.

In Chapter 7, we report a fabrication method of polymeric microlenses with a tunable contact angle based on the Ouzo effect. In addition, it is demonstrated that the obtained polymeric microlenses can improve the spatial imaging resolution for a standard optical microscope.

In Chapter 8, we present a method to generate micro indents in batches 
based on the Ouzo effect. The experimental results show that the size and depth of microdents can be tuned by the water concentration and PS concentration. Moreover, we confirm that the mass-produced microdents on a solid substrate can be used to obtain a lubricating surface.

Finally, we summarize the work presented in this thesis and give an outlook for future work. 


\section{Chapter 2}

\section{Giant plasmonic bubbles nucleation under different ambient pressures 1}

Water-immersed gold nanoparticles irradiated by a laser can trigger the nucleation of plasmonic bubbles after a delay time of a few microseconds [Wang et al., Proc. Natl. Acad. Sci. USA 122, 9253 (2018)]. Here we systematically investigated the light-vapor conversion efficiency, $\eta$, of these plasmonic bubbles as a function of the ambient pressure. The efficiency of the formation of these initial-phase and mainly water-vapor containing bubbles, which is defined as the ratio of the energy that is required to form the vapor bubbles and the total energy dumped in the gold nanoparticles before nucleation of the bubble by the laser, can be as high as $25 \%$. The amount of vaporized water first scales linearly with the total laser energy dumped in the gold nanoparticles before nucleation, but for larger energies the amount of vaporized water levels off. The efficiency $\eta$ decreases with increasing ambient pressure. The experimental observations can be quantitatively understood within a theoretical framework based on the thermal diffusion equation and the thermal dynamics of the phase transition.

\footnotetext{
${ }^{1}$ Published as: Binglin Zeng, Yuliang Wang, ${ }^{*}$ Mikhail E. Zaytsev, Chenliang Xia, Harold J. W. Zandvliet, ${ }^{*}$ and Detlef Lohse, ${ }^{*}$ Giant plasmonic bubbles nucleation under different ambient pressures, Phys. Rev. E 102, 063109 (2020).
} 


\subsection{Introduction}

Water-immersed noble-metal nanoparticles under irradiation of continuouswave lasers can rapidly produce large amounts of heat when the plasmon resonance frequency of the nanoparticle matches with the laser frequency, resulting in the explosive boiling of water surrounding the nanoparticles. This explosive boiling results in the nucleation and growth of so-called plasmonic bubbles [22, 30, 50-53]. These plasmonic bubbles are of great importance in numerous plasmonic-enhanced applications, ranging from cancer therapeutics [27, 54, 55], catalytic reactions [21], micromanipulation of nano-objects [56 58], and solvothermal chemistry [22]. They also have been proposed for the conversion of solar energy [23 26, 59 61]. In all these applications, light-induced vapor formation plays a key role. How efficiently the light can be converted into vapor during this process remains, however, unclear. The light-vapor conversion efficiency is related to the growth dynamics of the plasmonic bubbles as well as the physicochemical properties of the surrounding liquid [31].

Previous studies on plasmonic bubble formation and growth dynamics have mainly focused on the milliseconds to seconds timescale [24, 50, 52, 53]. Plasmonic bubbles formed on these timescales are hereafter referred to as ordinary plasmonic bubbles. In one of our previous studies we have shown that the growth of these ordinary plasmonic bubbles in water can be divided into two phases, a vaporization-dominated phase and a gas-diffusion dominated phase [62]. Plasmonic bubbles in the former phase have a smaller size. Water in the vicinity of the three-phase contact line is in direct contact with the laser spot. A relatively large fraction of the energy dumped in the nanoparticles is used to vaporize the surrounding water. As a result, these bubbles mainly contain vapor and exhibit a relatively high light-vapor conversion efficiency. In contrast, later the ordinary plasmonic bubbles contain both vapor and gas and are substantially larger. Therefore, the laser spots are then completely isolated from the water by the growing plasmonic bubbles [63]. Consequently, the heat at the laser spots cannot be directly transferred into the surrounding liquid. This significantly reduces the light-vapor conversion efficiency. As a result, the diffusion of dissolved gas expelled from the surrounding liquid dominates the growth of the plasmonic bubbles; consequently, they mainly contain gas and the light vapor conversion efficiency of this phase is substantially lower than in the vapor-dominated phase.

We recently analyzed the very initial plasmonic bubble phase on a time scale of microseconds [30]. In this very initial phase a giant plasmonic bubble 
forms after a short delay time after switching on the laser, with a growth rate that is about three orders of magnitude larger than the ordinary plasmonic bubbles [30]. The lifetime of these initial phase plasmonic bubbles is, however, very short. Shortly after their formation they collapse due to the condensation of vapor [30].

The relatively large light-vapor conversion efficiency and the explosive growth rate of the giant initial plasmonic bubbles makes them very interesting for numerous applications. However, the underlying mechanism for their formation, as well as the light-vapor conversion process during bubble nucleation, are not quantitatively understood yet. Among the various physicochemical properties of the liquid such as the latent heat, obviously also the boiling point is very relevant for the nucleation and formation of plasmonic bubbles. However, it is very challenging to tune the boiling point of a liquid without changing the other physicochemical properties. Here we have varied the boiling point of water from 100 to $175{ }^{\circ} \mathrm{C}$ by changing the ambient pressure from 1 to 9 bar. We have studied the nucleation and growth of the initial giant plasmonic bubbles under different ambient pressures and laser powers in order to obtain a thorough and solid understanding of the bubble nucleation as well as the light-vapor conversion processes.

\subsection{Experimental system}

\subsubsection{Sample preparation}

A gold layer of $\sim 45 \mathrm{~nm}$ was deposited on an amorphous fused-silica wafer by using an ion-beam sputtering system (home-built $\mathrm{T}^{\prime}$ COathy machine, MESA ${ }^{+}$, Twente University). The wafer was coated with a bottom antireflection coating (BARC) layer $(\sim 186 \mathrm{~nm})$ and a photoresist $(\mathrm{PR})$ layer $(\sim 200 \mathrm{~nm})$. Periodic nanocolumns with diameters of $\sim 110 \mathrm{~nm}$ were patterned in the PR layer by using displacement Talbot lithography (PhableR 100C, EULITHA) [64]. Subsequently, these periodic PR nanocolumns were transferred to the underlying BARC layer, forming 110-nm BARC nanocolumns by using nitrogen plasma etching (home-built TEtske machine, NanoLab) at 10 mTorr and $25 \mathrm{~W}$ for 8 min. Taking these BARC nanocolumns as a mask, the Au layer was then etched by ion-beam etching (Oxford i300, Oxford Instruments, United Kingdom) with 5 -sccm Ar and 50-55 mA at an inclined angle of $5^{\circ}$. The etching for 9 min resulted in periodic Au nanodots supported on cone-shaped fused-silica features. The remaining BARC was stripped using oxygen plasma for $10 \mathrm{~min}$ 
(TePla 300E, PVA TePla AG, Germany). The fabricated array of Au nanodots was annealed to $1100{ }^{\circ} \mathrm{C}$ in $90 \mathrm{~min}$. and subsequently cooled passively to room temperature. During the annealing process, these Au nanodots reformed into spherical-shaped Au nanoparticles, as shown in Figs. 2.1(a) and (b).

(a)
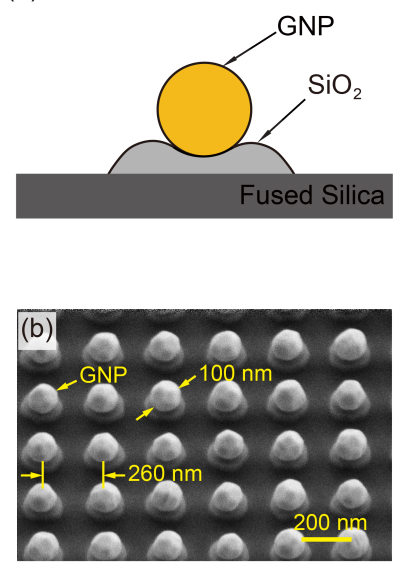

(c)

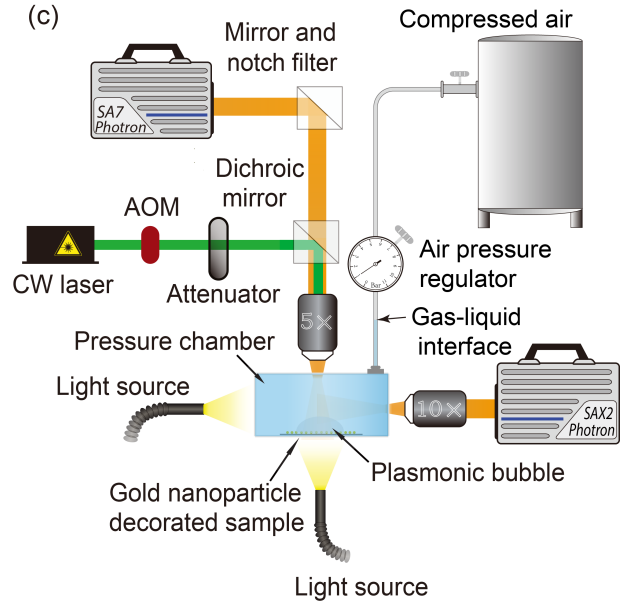

Figure 2.1: (a) Schematic of a gold nanoparticle sitting on a $\mathrm{SiO}_{2}$ island. (b) A scanning electron microscopy image of the gold nanoparticle decorated substrate. (c) Schematic of the optical-imaging facilities for giant initial bubble observation under different ambient pressures. A pressure chamber is used to tune the pressure from 1 to 9 bar. A narrow tube together with an elevated gas-liquid interface significantly slows down gas diffusion from the compressed air to the water in the pressure chamber. As a result, the gas concentration of the water in the pressure chamber remains almost constant throughout the experiments.

\subsubsection{Setup description}

Figure 2.1(c) shows a schematic diagram of the experimental setup used for the study of initial giant bubbles under different ambient pressures $p_{0}$. In the setup, the gold nanoparticle decorated substrate was placed in a homebuilt pressure chamber. The chamber was completely filled with deionized (DI) water (Milli-Q Advantage A10 System, Germany) and connected to the compressed air via a narrow tube. Before the experiments, the DI water was exposed to air for $24 \mathrm{~h}$ to obtain fully air-saturated water. The gas concentration in the DI water was measured by an oxygen meter (Fibox 3 
Trace, PreSens). The measured relative air concentration level was 0.99. The pressure of the chamber was tuned by an air-pressure regulator. Here we have used seven different ambient pressures of 1, 2, 3, 4, 5, 7, and 9 bar, respectively. A continuous-wave laser (Cobolt Samba) with a wavelength of $532 \mathrm{~nm}$ was used for irradiation of our samples. The radius $R_{\ell}$ of the laser spot was about $12.5 \mu \mathrm{m}$. The laser power $P_{\ell}$ projected on the sample surface was tuned via two polarization filters and measured by a photodiode power sensor (S130C, ThorLabs). Laser pulses of $10 \mathrm{~ms}$ were generated by a pulse-delay generator (BNC model 565).

Two high-speed cameras were used for top view and side-view imaging, respectively. The top-view camera (SA7, Photron) was used to focus the laser on the sample surface, while the side-view camera (SAX2, Photron) was used for the observation of the formation of bubbles. The top-view camera and the side-view camera were equipped with a $5 \times$ (LMPLFLN, Olympus) and a $10 \times$ (SLMPLN, Olympus) long working distance objectives, respectively. A frame rate of $540 \mathrm{kfps}$ was used for side-view imaging. A home-designed algorithm was applied to segment the acquired bubble images [65 67]. With this algorithm, the bubble volume can be extracted automatically.

\subsubsection{Results and discussion}

Several images of initial giant bubbles at their maximum size at the same laser power $P_{f}$ of $32.7 \mathrm{~mW}$, but under different ambient pressures, $p_{0}$, are shown in Fig. 2.2(a). These results show that the maximum size of the giant bubble rapidly decreases with increasing $p_{0}$, reflecting that with increasing ambient pressure $p_{0}$ the expanding has to do more work against the ambient pressure. As we have previously reported, the volume of the initial phase giant bubbles is directly related to the delay time, $\tau_{d}$, which is defined as the time interval between switching on the laser and the nucleation of the bubble [30]. In Fig. 2.2(b) a semilogarithmic plot of $\tau_{d}$ as a function of laser power $P_{\ell}$ is shown. As already seen in Ref. [30], the delay time, $\tau_{d}$, decreases with increasing laser power $P_{\ell}$, but here we find that $\tau_{d}$ is independent of $p_{0}$ for a fixed $P_{\ell}$ in the range of 1 to 9 bar; see Fig. 2.2(c). We noticed that the measured delay time for all three laser powers under 5 bar is relatively higher than for the other pressure values. We speculate that this is a systematic error, presumably originating from the laser spot under 5 bar being slightly out of focus, leading to a slightly lower laser power density and hence increased delay time.

Before bubble nucleation, the water has to be heated up to the nucleation temperature $T_{n}$, which usually substantially exceeds the boiling temperature 

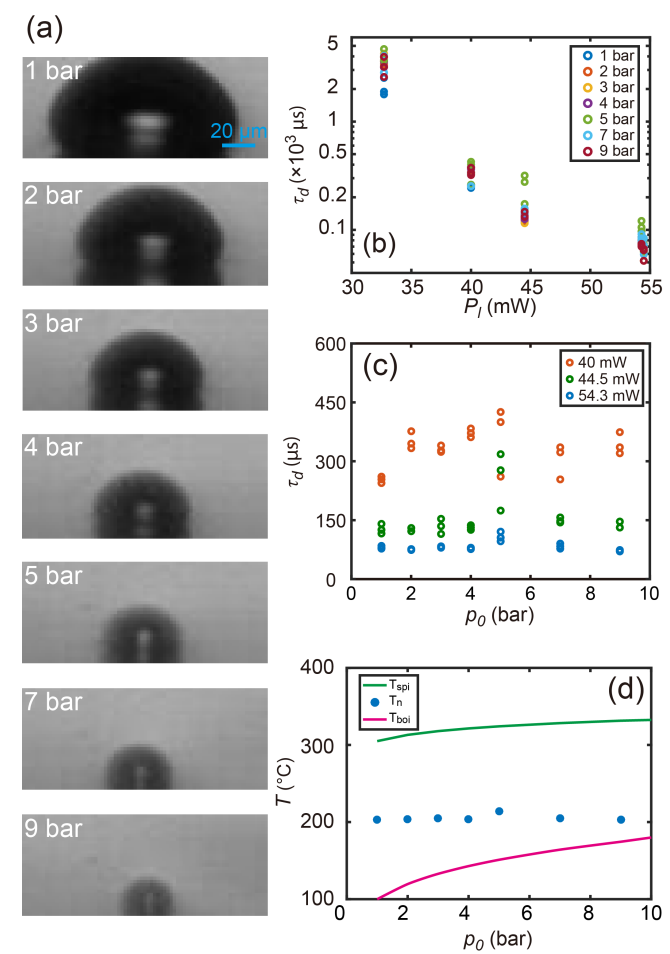

Figure 2.2: (a) Examples of side-view images of initial giant bubbles at the same laser power $P_{\ell}=32.7 \mathrm{~mW}$, but at different ambient pressures $p_{0}$ (see legend). The respective snapshots were taken at the maximum of the bubble expansion. (b) Delay time $\tau_{d}$ vs $P_{\ell}$ at different ambient pressures. (c) Delay time $\tau_{d}$ vs $p_{0}$ at different values of $P_{\ell}$ (see legend). (d) Bubble nucleation temperature $T_{n}$ vs the ambient pressure $p_{0}$. The nucleation temperature $T_{n}$ is obtained by fitting $\tau_{d}$ with the numerical model. It is found to be independent of $p_{0}$. We also show the spinodal temperature $T_{s p i}$, i.e., the theoretical maximal attainable temperature of the liquid without vapor bubble nucleation.

$T_{\text {boil }}[68-70]$. The higher the laser power $P_{\ell}$, the faster the surrounding water heats up and the shorter the delay time $\tau_{d}$. The nucleation temperature $T_{n}$ can be numerically determined; for details, see Refs. [30, 31]. The spatialtemporal evolution of the temperature of water, $T(r, t)$, surrounding a gold nanoparticle that is heated by a laser can be numerically calculated by solving 
the heat diffusion equation,

$$
\partial_{t}(T(r, t))=\frac{P_{l}(r, t)}{\rho c_{p}}+\kappa \frac{1}{r^{2}} \partial_{r}\left(r^{2} \partial_{r} T(r, t)\right),
$$

where $\kappa, \rho$, and $c_{p}$ are thermal diffusivity, density, and heat capacity of water, $r$ is the distance to the nanoparticle, $t$ is the time, and $P_{\ell}(r, t)$ the laser power density (in $\mathrm{W} / \mathrm{m}^{3}$ ). For the numerical solution of the partial differential Eq. 2.1, as spatial boundary condition we took the specific configuration of the gold nanoparticle decorated sample surface used in the experiment. The heat conductivity of water and fused silica are 0.61 and $1.38 \mathrm{~W} /(\mathrm{mK})$, respectively. This simple thermal diffusion model does not include the interfacial thermal resistance term (Kapitza), which does not play a role here because our timescale exceeds the timescale of the study reported in Ref. [71] (where it is considered) by several orders of magnitude. The temperature field $T(r, t)$ generated by an array of nanoparticles can be considered as the linear superposition of the temperature distribution fields of the individual gold nanoparticles within a Gaussian laser beam profile,

$$
T(x, y, z, t)=\sum_{i=1}^{N_{n p}}\left[T_{i}\left(d_{i,(x, y, z)}, t\right)\right],
$$

where $N_{n p}$ is the number of gold nanoparticles under laser irradiation, $T_{i}$ is the temperature field produced by the $i_{t h}$ nanoparticle, and $d_{i}$ is the distance to the center of the $i_{t h}$ nanoparticle. Note that the delay time before the initial plasmonic bubble nucleation is more than $50 \mu \mathrm{s}$, which is much longer than the thermal relaxation time of $10 \sim 100 \mathrm{ps}$ for the electrons in the metal nanoparticles mentioned in Ref. [72]. Therefore, the thermal relaxation effect of the GNPs close to the border of the laser beam can be neglected in this system.

As shown in Ref. [30], by numerically solving Eqs. 2.1 and 2.2, one can directly obtain the time required to reach the nucleation temperature of water at a given laser power. This approach was employed for all the experimental data using a root-mean-square minimization method. In this way, $T_{n}$ was obtained for different ambient pressures and is shown in Fig. 2.2(d). Interestingly, $T_{n}$ is independent of the ambient pressure and has values around 200 ${ }^{\circ} \mathrm{C}$.

In addition, the results shown in Figs. 2.2(b)-(d) also provide insight into the dependence of $T_{n}$ on the amount of dissolved gas in water. Previous studies have shown that $\tau_{d}$ strongly depends on the gas concentration in water [30, 
32]. In the experiments, the absolute gas concentration is independent of the ambient pressure, $p_{0}$, as we do not give the water the time to be equilibrated after changing the ambient pressure. We, therefore, arrive at the conclusion that the nucleation temperature mainly depends on the absolute amount of dissolved gas in the water, which here does not depend on $p_{0}$.
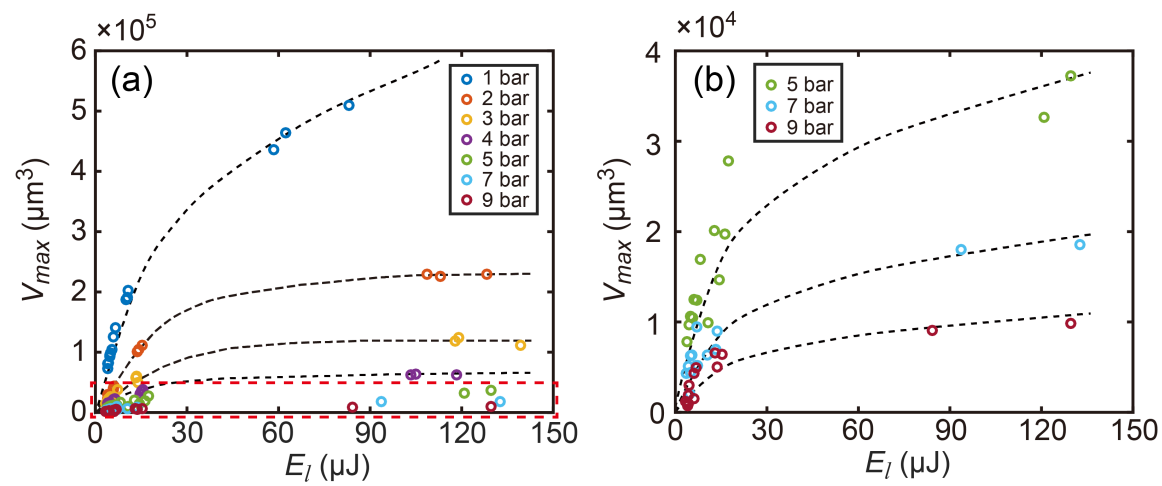

Figure 2.3: (a) Maximum volume $V_{\max }$ of the bubbles as a function of the total deposited laser energy $E_{\ell}$ under different ambient pressures $p_{0}$ (see legend) ranging from 1 to 9 bar. (b) Zoomed-in version of the same plot curves for $p_{0}=5,7$, and 9 bar [red dashed box in (a)]. All curves exhibit two regimes, namely a linear regime and a nonlinear regime, irrespective of the value of $p_{0}$. The dashed lines are drawn to guide the eye.

The maximum volume $V_{\max }$ of the bubbles as a function of the total deposited energy $E_{\ell}=P_{\ell} \tau_{d}$ for different values of $p_{0}$ is shown in Fig. 2.3(a). Figure 2.3.(b) shows three curves [enclosed by the red dashed box in Fig. 2.3. (a)] for ambient pressures of 5, 7, and 9 bar, respectively. One can see that, regardless of the exact value of $p_{0}$, all curves exhibit a qualitatively $V_{\max }\left(E_{\ell}\right)$ dependence. When $E_{\ell}$ is smaller than $20 \mu \mathrm{J}, V_{\max }$ linearly increases with $E_{\ell}$, which is consistent with our previous study [30,32]. However, when $E_{\ell}$ is larger than $20 \mu \mathrm{J}, V_{\max }\left(E_{\ell}\right)$ dependence becomes nonlinear.

In the linear regime, the amount of water vapor in the bubbles is proportional to $E_{\ell}$. The proportionality factor $k$ between energy and maximum bubble volume can be used to estimate the light-vapor conversion efficiency $\eta$. The linear regime of the $V_{\max }\left(E_{\ell}\right)$ curves for different values of $p_{0}$ are shown in Fig. 2.4(a). The extracted proportionality factor $k$ as a function of $p_{0}$ is shown in Fig. 2.4(b). It can be seen that $k$ rapidly decreases from $1.9 \times 10^{4}$ $\mu \mathrm{m}^{3} / \mu \mathrm{J}$ to $440 \mu \mathrm{m}^{3} / \mu \mathrm{J}$ when $p_{0}$ is increased from 1 to 9 bar. 

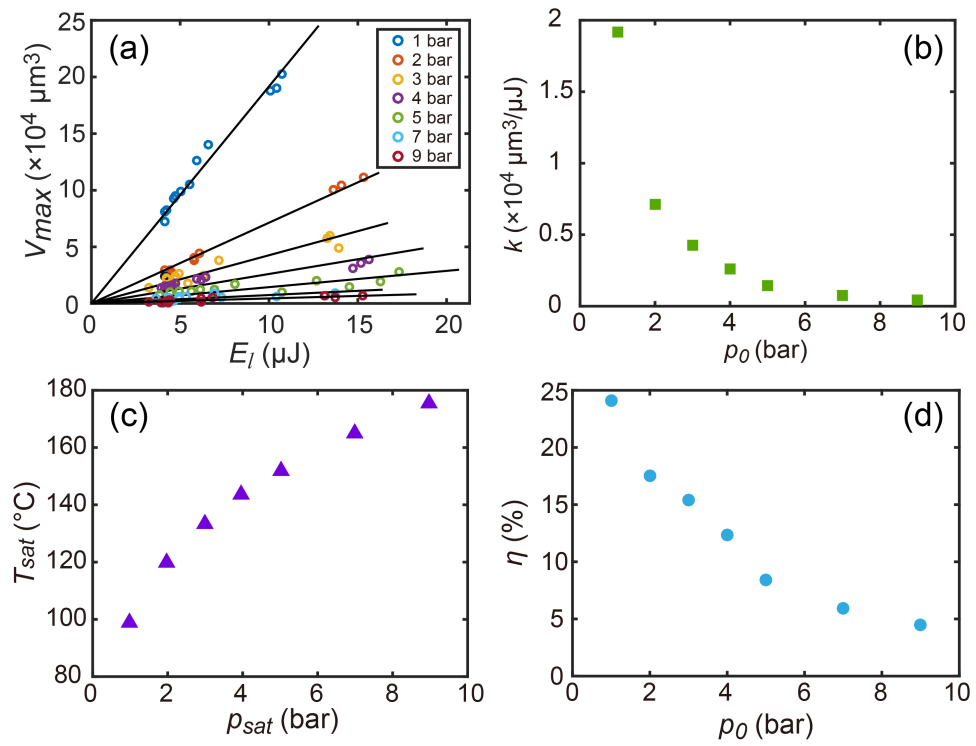

Figure 2.4: (a) The maximum volume $V_{\max }$ of the giant bubbles as a function of $E_{\ell}$ in the linear regime under different ambient pressures $p_{0}$ (see legend). (b) The prefactor $k=V_{\max } / E_{\ell}$ of the linear relation $V_{\max }$ vs $E_{\ell}$ as a function of $p_{0}$. (c) Saturation temperature $T_{\text {sat }}$ vs saturation pressure, $p_{\text {sat }}$, at the moment of maximum giant bubble volume under different ambient pressure $p_{0}$. (d) Experimentally obtained light-vapor conversion efficiencies $\eta$ vs the ambient pressure $p_{0}$.

We now define the efficiency $\eta$ as the ratio of the energy $E_{b}$ used for water vaporization during vapor bubble formation to the energy $E_{d}$ deposited in the gold nanoparticles before nucleation of the bubble, i.e., $\eta=E_{b} / E_{d}$. Considering a gold nanoparticle coverage of $\xi=11.6 \%$, we have $E_{d}=\xi E_{\ell}$, where $E_{\ell}$ is the total deposited laser energy on the sample surface. The value $\eta$ can then be written as

$$
\eta=\frac{E_{b}}{\xi E_{\ell}}
$$

The energy $E_{b}$ required to vaporize the water is composed of two components. One component is the energy needed to heat the water to vaporization temperature and the other component deals with the phase transition of the water from liquid to vapor, i.e., the latent heat $H_{v a p}$. Consequently, $E_{b}$ for a vapor bubble is given by 


$$
E_{b}=\left(\int_{T_{0}}^{T_{\mathrm{sat}}} c_{p} d T+H_{\mathrm{vap}}\right) \frac{M p_{\mathrm{sat}} V_{\mathrm{max}}}{R_{g} T_{\mathrm{sat}}},
$$

where $T_{0}$ and $T_{\text {sat }}$ are the ambient temperature and saturation temperature of water, respectively. $M$ is the molar mass of water $(18 \mathrm{~g} / \mathrm{mol})$ and $p_{\text {sat }}$ is the saturation pressure of water vapor at the moment that the bubble reaches its maximum volume. $V_{\max }$ is the maximum volume of the bubble and $R_{g}=$ $8.314 \mathrm{~J} /(\mathrm{mol} \mathrm{K})$ the universal gas constant. By combining Eqs. 2.3 and 2.4, we find

$$
\eta=\left(\int_{T_{0}}^{T_{\mathrm{sat}}} c_{p} d T+H_{\mathrm{vap}}\right) \frac{M p_{\mathrm{sat}} V_{\mathrm{max}}}{R_{g} T_{\mathrm{sat}} \xi E_{l}} .
$$

To calculate $\eta$ from Eq. 2.5, we note that the saturation pressure $p_{\text {sat }}$ is close to the ambient pressure $p_{0}$ and can be estimated to be $p_{0}-0.04$ bar [30]. Once $p_{\text {sat }}$ is determined, $T_{\text {sat }}$ can be obtained [Fig. 2.4(c)] [73]. The ratio $p_{\text {sat }} / T_{\text {sat }}$ is dependent on $p_{0}$. The efficiency can be obtained using the prefactor $k$ for the linear regime in the $V_{\max }\left(E_{\ell}\right)$ dependence at a given $p_{0}$. The obtained efficiency as a function of $p_{0}$ is shown in Fig. 2.4(d). The efficiency decreases from $25 \%$ to $5 \%$ when $p_{0}$ is increased from 1 to 9 bar.

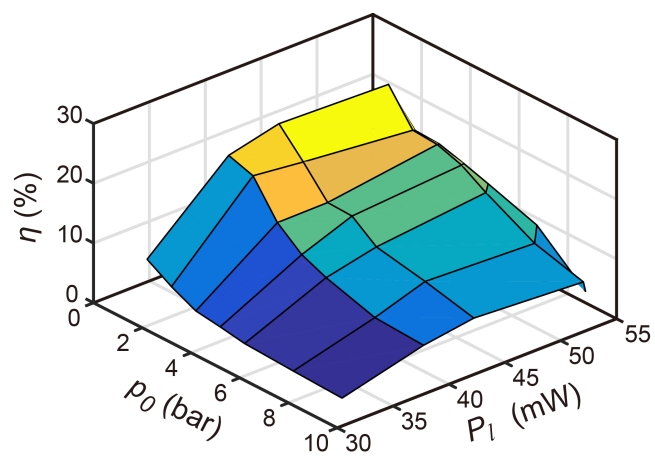

Figure 2.5: The experimentally measured light-vapor conversion efficiency, $\eta$, as a function of laser power $P_{\ell}$ and ambient pressure $p_{0}$.

In the nonlinear regime of the $V_{\max }\left(E_{\ell}\right)$ dependencies, $k$ is still defined as $V_{\max } / E_{\ell}$ and obviously depends on $E_{\ell}$ and also $p_{0}$. Following Eq. 2.5, the light-vapor conversion efficiency changes accordingly. For the experimental results shown in Fig. 2.3, the corresponding efficiency as a function of laser 
power $P_{\ell}$ and ambient pressure $p_{0}$ is presented in Fig. 2.5, revealing that the efficiency decreases with increasing $p_{0}$ and decreasing $P_{\ell}$.

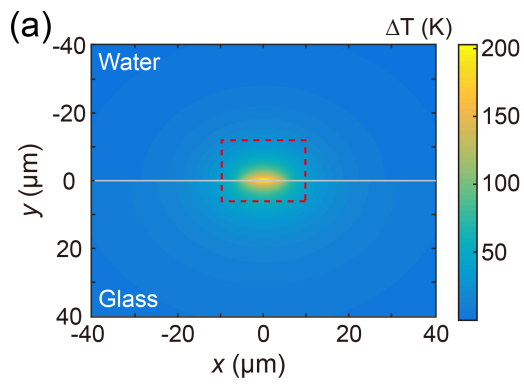

(b)

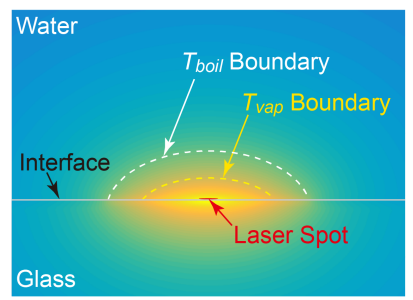

(c)

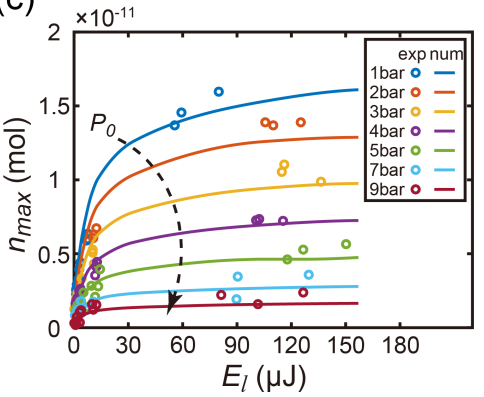

(d)

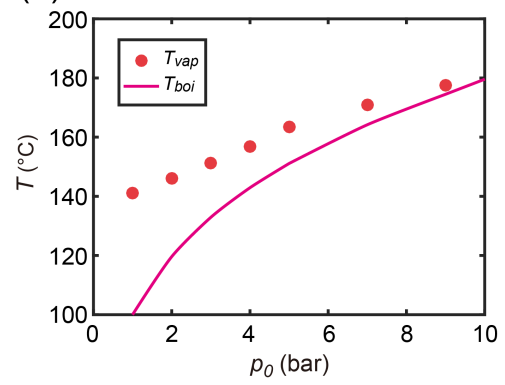

Figure 2.6: (a) Water temperature field in the vicinity of a laser spot constructed by numerically solving the model discussed in the text. (b) Enlarged view of the water temperature field [dashed red box in panel (a)]. Once the water temperature at the impact point of the laser reaches $T_{n}$, water within the regime with boundary of $T_{\text {vap }}$ rapidly vaporizes and a giant bubble is nucleated. (c) Experimentally measured (data points) and numerically calculated (curve) amount of vaporized water in moles, $n_{\max }$, as a function of the total deposited laser energy $E_{\ell}$ for various ambient pressures $p_{0}$ (see legend). (d) Vaporization temperature $T_{\text {vap }}$ and boiling point $T_{\text {boil }}$ vs ambient pressure $p_{0}$.

To better understand how $P_{\ell}$ and $p_{0}$ affect $\eta$ during the nucleation of the initial phase giant bubbles, we numerically solve Eqs. 2.1 and 2.2 for a whole range of $P_{\ell}$ and $p_{0}$. An example of the constructed temperature distribution field is shown in Fig. 2.6.(a). From this figure, one can see that the temperature of the water rapidly decreases with increasing distance away from the center of the laser spot. In our model, we assume that the following two conditions are valid during the nucleation of the bubble: (1) the bubble starts to nucleate when the highest temperature of the surrounding water has 
reached the nucleation temperature $T_{n},(2)$ the volume of the bubble is determined by the amount of water that has a temperature higher than an ambient pressure-dependent threshold temperature, which is defined as vaporization temperature $T_{\text {vap }}$. In Fig. 2.6.(b), a zoom-in plot of the temperature distribution of the red dashed box in Fig. 2.6(a) is shown. The key question is of course how to determine $T_{\text {vap }}$.

Given a certain water temperature distribution, the value $T_{\text {vap }}$ determines the amount of water that can be vaporized in case a bubble nucleates. A higher value of $T_{\text {vap }}$ implies a smaller volume of water and thus a smaller bubble. Therefore, the maximum size of the bubble allows us to determine $T_{\text {vap }}$. The amount of moles of vaporized water molecules $n_{\max , \exp }$ in a giant bubble is given by

$$
n_{\text {max }, \exp }=\frac{p_{\text {sat }} V_{\text {max }}}{R_{g} T_{\text {sat }}},
$$

$n_{\text {max }, \text { exp }}$ as a function of $E_{\ell}$ for different pressures $p_{0}$ is plotted in Fig. 2.6(c) (circles refer to the experimental data). It clearly shows that for a given laser energy $E_{\ell}$ the amount of vaporized water decreases with increasing $p_{0}$. For higher values of the laser energy $E_{\ell}, n_{\max , \exp }$ levels off. The above two observations are consistent with the obtained $\eta\left(P_{\ell}\right)$ and $\eta\left(p_{0}\right)$ dependencies.

The thermal diffusivity $\kappa$ and specific heat capacity $c_{p}$ of water only very weakly depend on the ambient pressure (Table 3.1 in the Appendix). We have shown that the nucleation temperature $T_{n}$ is independent of the ambient pressure. Based on these dependencies the water temperature distribution is independent of the ambient pressure $p_{0}$ for a fixed laser power $P_{\ell}$. The temperature distribution in the water depends, however, on the laser power. Upon selecting a value for $T_{\text {vap }}$ the amount of water molecules can be calculated, which implies that we can extract $n\left(T_{v a p}\right)$ for each laser power. The total deposited laser energy in the numerical calculation is given by $E_{\ell}=P_{\ell} \tau_{d}$. Subsequently, the $n\left(E_{\ell}\right)$ dependence can be obtained by gradually tuning $T_{\text {vap }}$ from room temperature to $T_{n}$ for each value of $P_{\ell}$. Using the $n\left(E_{\ell}\right)$ dependence, $T_{v a p}\left(p_{0}\right)$ can be determined by minimizing $\Sigma\left[n\left(E_{\ell}\right)-n_{\max , \exp }\left(E_{\ell}\right)\right]^{2}$. The results are displayed in Fig. 2.6.(c). The numerically determined $n_{\max }\left(E_{\ell}\right)$ dependence agrees well with the experimentally obtained results.

The numerically determined $T_{\text {vap }}$ for different ambient pressures is shown in Fig. 2.6(d). The solid circles refer to the numerically determined $T_{\text {vap }}$, while the solid curve represents the water boiling point $T_{b o i l}$ as a function of $p_{0}$. It is clear that $T_{\text {vap }}$ is in between $T_{\text {boil }}$ and $T_{n}$ [around $200{ }^{\circ} \mathrm{C}$, as shown in Fig. 2.2(d)]. With increasing $p_{0}$, the vaporization temperature $T_{\text {vap }}$ gets 
closer to $T_{n}$.

We now return to the observed dependencies of $\eta$ on the laser power $P_{\ell}$ and the ambient pressure $p_{0}$. A higher laser power $P_{\ell}$ leads to a faster increase of the water temperature and to a short delay time $\tau_{d}$. If $\tau_{d}$ is small compared to the thermal diffusion timescale $\tau_{\text {diff }} \approx R_{\ell}^{2} /(\pi \kappa)$, only a small amount of energy can diffuse into the nonvaporizable zone, resulting in a high efficiency. On the contrary, a lower laser power $P_{\ell}$ leads to a longer delay time $\tau_{d}$. Since the thermal diffusivity $\kappa$ is almost independent of the ambient pressure, an increased delay time $\tau_{d}$ results in an increased amount of energy diffusion into the nonvaporization zone and hence a lower efficiency. Regarding the ambient pressures $p_{0}$, a higher value will lead to an increased vaporization temperature $T_{\text {vap }}$. As a result, a reduced portion of heated water will be vaporized. Although the delay time of bubble nucleation remains constant for different ambient pressures, the portion of laser energy used for water vaporization decreases, resulting in a decreased light-vapor conversion efficiency.

\subsection{Conclusions}

We have systematically investigated the nucleation of initial giant plasmonic bubbles in water with boiling points ranging from 100 to $175{ }^{\circ} \mathrm{C}$ by tuning ambient pressure from 1 to 9 bar. The experimental observations can be quantitatively understood within a theoretical framework based on the thermal diffusion equation and the thermodynamics of the phase transition. It has shown that water in the vicinity of laser-irradiated gold nanoparticles can be divided into a vaporization zone and a nonvaporization zone. The two zones are divided by vaporization temperatures, above which water will be vaporized during the giant bubble nucleation. Water in the vaporization zone vaporizes when the bubble nucleation temperature is reached. This bubble nucleation temperature only depends on the absolute amount of gas dissolved in the water, while the vaporization temperature increases with water boiling points. As a result, the light-vapor conversion efficiency decreases with increasing boiling points.

This study of the light-vapor conversion efficiency of laser-irradiated $\mathrm{Au}$ nanoparticles in water is also relevant for applications. For example, noblemetal nanoparticles are one of the most commonly used solar energy absorbers. Our study demonstrates that the interfacial (localized) heating can significantly increase the solar-vapor conversion efficiency [74-76]. 


\subsection{Appendix}

\subsubsection{Physicochemical properties of water under different am- bient pressures}

The physicochemical properties of pure water under ambient pressures of 1 bar and 10 bar are listed in Table 2.1. The results show that density $\rho$, thermal conductivity $\lambda$, thermal diffusivity $\kappa$, latent heat of vaporization $H_{\text {vap }}$, and specific heat capacity $c_{p}$ of pure water at 10 bar are very close to that at 1 bar. Therefore, we can assume that the above 4 parameters of water basically remain constant when ambient pressure changes from 1 to 9 bar.

Table 2.1: Physicochemical properties of water under ambient pressure of 1 and 10 bar [73]. All values are taken at $25^{\circ} \mathrm{C}$.

\begin{tabular}{lcc}
\hline Parameter & 1 bar & 10 bar \\
\hline Density $\rho\left(\mathrm{kg} / \mathrm{m}^{3}\right)$ & 997.05 & 1000.3 \\
Thermal conductivity $\lambda[\mathrm{W} /(\mathrm{m} \mathrm{K})]$ & 606.52 & 610.0 \\
Thermal diffusivity $\kappa\left(\mathrm{m}^{2} / \mathrm{s}\right)$ & 0.146 & 0.145 \\
Latent heat of vaporization $H_{\text {vap }}(\mathrm{kJ} / \mathrm{kg})$ & 104.92 & 113.48 \\
Specific-heat capacity $c_{p}[\mathrm{~kJ} /(\mathrm{kg} \mathrm{K})]$ & 4.1813 & 4.1973 \\
\hline
\end{tabular}




\section{Chapter 3}

\section{Plasmonic bubble nucleation in binary liquids 1}

Metal nanoparticles under laser irradiation can produce enormous heat due to surface plasmon resonance. When submerged in a liquid this can lead to the nucleation of plasmonic bubbles. In the very early stage, the nucleation of a giant vapor bubble was observed with an ultrahigh-speed camera. In this study, the formation of this giant bubble on gold nanoparticles (GNPs) in six binary liquid combinations has been investigated. We find that the time delay between the beginning of the laser heating and the bubble nucleation is determined by the absolute amount of dissolved gas in the liquid. Moreover, the bubble volume mainly depends on the vaporization energy of the liquid, consisting of the latent heat of vaporization and the energy needed to reach the boiling temperature. Our results contribute to controlling the initial giant bubble nucleation and have strong bearings on applications of such bubbles.

\footnotetext{
${ }^{1}$ Published as: Marvin Detert, Binglin Zeng, Yuliang Wang, ${ }^{*}$ Hai Le The, Harold J. W. Zandvliet, and Detlef Lohse, ${ }^{*}$ Plasmonic bubble nucleation in binary liquids, J. Phys. Chem. C 124, 2591-2597 (2020). Detert and Zeng contributed equally to this work.
} 


\subsection{Introduction}

When irradiated by a continuous-wave laser, gold nanoparticles (GNPs) immersed in a liquid can produce huge amounts of heat due to the surface plasmon resonance. The enormous heat can vaporize the surrounding liquid, leading to the nucleation of so-called plasmonic vapor bubbles [23, 51, 58, 62, 77, 78]. These bubbles have shown potential for various future applications. They range from medical ones, such as drug delivery to a single cell [55] and cancer therapy [79-83], to devices on microfluidic chips like micro-pumps and -valves [84]. Another interesting approach is plasmon-assisted catalysis for steam reforming ethanol [21]. It has been demonstrated that a microfluidic channel decorated with GNPs and irradiated by a laser can form the catalytic reaction products $\mathrm{H}_{2}$, $\mathrm{CO}$ and $\mathrm{CO}_{2}$, using GNPs as a catalyst and heat source simultaneously $[21,22,85]$. Understanding the nucleation and growth dynamics of the plasmonic bubbles in multicomponent liquids is thus essential to develop and further improve this multitude of applications.

In our recent study, the nucleation of an initial giant bubble in pure liquids was found in the very early stage of the plasmonic bubble generation via ultrahigh-speed imaging. [30, 31]. This bubble shows extraordinary characteristics, such as a short lifetime and an explosive growth rate. In particular, the rapid growth is essential for applications, e.g. for the aforementioned cell therapy, and might also be used in other future applications. However, the nucleation process is not well understood yet. This is partially due to the complexity of the system and partially due to the short time scales on which the nucleation takes place. A preliminary study has shown that the delay between the beginning of the laser heating and the nucleation in water is affected by both the laser power and the relative gas concentration [30]. Additionally, these bubbles have also been studied in n-alkanes [31]. There, a decrease of the bubble volume has been observed for an increase in the carbon chain length of the n-alkane. Moreover, the nucleation takes place faster in all investigated n-alkanes than in water. Although water and six different pure n-alkanes have been investigated, a detailed understanding of the nucleation mechanism is still lacking.

In order to better understand the bubble nucleation, it is necessary to experimentally explore the effect of multiple parameters, such as the gas concentration, boiling point, thermal conductivity, heat capacity of the liquid and the latent heat of vaporization. However, these parameters are hard to tune for pure liquids. This difficulty led us to binary liquids, as their parameters can 
easily be tuned via the mixing ratio and hence allow to explore a wider range of parameters. Therefore, we have systematically investigated six binary liquids, namely to disentangle the relevance and influence of various parameters on the nucleation of the initial giant bubble. We are particularly interested in finding the determining parameters for the nucleation time and volume of the bubble, because those will have strong bearings on the applications mentioned above.

\subsection{Methods and Materials}

\subsubsection{Binary liquids preparation}

In order to study a wide variety of parameters, multiple binary liquids with varying composition were investigated. In our experiment, ethanol, acetone, 1-propanol, 2-propanol, 1-butanol from Sigma-Aldrich and MiliQ water were employed to prepare the binary liquids. Before mixing, the pure liquids were exposed to air for more than one day to saturate them. Water was mixed with acetone, ethanol and 1-propanol. Additionally, ethanol mixed with acetone, 2propanol and 1-butanol. Each combination was measured for multiple mixing ratios including the pure liquids. The parameters of the pure liquids are listed in Table 3.1. The parameters of the binary liquids were estimated by weighting the pure liquid parameters with their mole fraction in the mixture. Since the pure liquids were saturated in air, the amount of dissolved gas in the binary liquids can be estimated by $c_{g}=0.79 S\left(\mathrm{~N}_{2}\right)+0.21 S\left(\mathrm{O}_{2}\right)$, where $S\left(\mathrm{~N}_{2}\right)$ and $S\left(\mathrm{O}_{2}\right)$ are the solubility of $\mathrm{N}_{2}$ and $\mathrm{O}_{2}$ in the liquid, respectively.

Table 3.1: Parameters of the pure liquids. All values are given at ambient pressure. The surface tensions and solubilities are those at room temperature. All other parameters are those at the respective boiling points of the liquid [86-90].

\begin{tabular}{lcccccc}
\hline Parameter & Water & Ethanol & Acetone & 1-Propanol & 2-Propanol & 1-Butanol \\
\hline Boiling point $\left({ }^{\circ} \mathrm{C}\right)$ & 100 & 78 & 56 & 97 & 82 & 118 \\
Heat capacity $(\mathrm{J} /(\mathrm{mol} \mathrm{K}))$ & 75.9 & 146.6 & 129.4 & 192.7 & 205.2 & 249.8 \\
Thermal cond. $(\mathrm{W} /(\mathrm{m} \mathrm{K}))$ & 0.679 & 0.153 & 0.143 & 0.138 & 0.125 & 0.135 \\
Latent heat of vap. $(\mathrm{kJ} / \mathrm{mol})$ & 40.7 & 39.2 & 29.1 & 41.3 & 39.9 & 43.3 \\
Surface tension $(\mathrm{mN} / \mathrm{m})$ & 72.8 & 22.4 & 23.7 & 23.7 & 21.4 & 24.5 \\
Nitrogen solubility $\left(\mathrm{V}_{g} / \mathrm{V}_{l}\right)$ & 0.018 & 0.149 & 0.184 & 0.133 & 0.147 & 0.122 \\
Oxygen solubility $\left(\mathrm{V}_{g} / \mathrm{V}_{l}\right)$ & 0.035 & 0.244 & 0.267 & 0.221 & 0.246 & 0.190 \\
\hline
\end{tabular}




\subsubsection{Setup description}

The preparation of sample with gold nanoparticle is shown shown in Figs. 2.1 (a) and (b) in Chapter 2. Figure 3.1 shows the experimental setup for the plasmonic microbubble imaging. The gold nanoparticle decorated sample is placed in a glass cuvette, submerged in a liquid and irradiated by a $300 \mathrm{~mW}$, $532 \mathrm{~nm}$ continuous wave laser (Cobolt Samba). The light intensity at the substrate is controlled via a halfwave plate and a polarizer and measured by a photodiode power sensor (S130C, ThorLabs). An acoustic-optic modulator acts as a triggerable shutter. The laser spot diameter is $10 \mu \mathrm{m}$ and the intensity can be varied between 0 and $200 \mathrm{~mW}$. Laser pulses of $400 \mu$ s were generated and controlled by a pulse/delay generator (BNC model 565) in order to study the short-term dynamics of the microbubbles. Two high-speed cameras were installed in the setup, one for the top view and another for the side view. The top view camera (SA7) is equipped with a $5 \times$ long working distance objective (LMPLFLN, Olympus). The side view camera (Photron SAZ) can be equipped with two long working distance objectives, $10 \times$ (LMPLFLN, Olympus) or $20 \times$ (SLMPLN, Olympus), and can be operated at frame rates up to $1200 \mathrm{kfps}$. Two light sources, a Sumita LS-M350 and a Schott ACE I, provided back light illumination for the high-speed cameras.

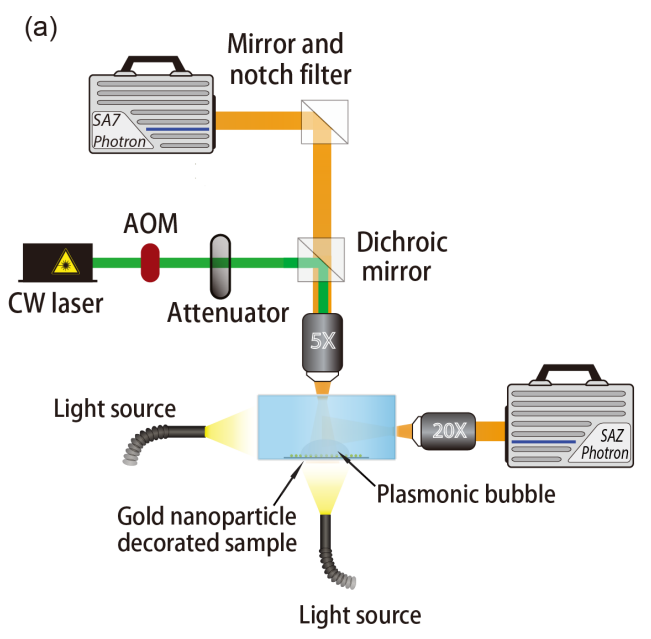

Figure 3.1: (a) Schematic of the optical imaging facilities for plasmonic microbubble observation; 


\subsection{Results and discussion}

The nucleation and explosive growth of a giant bubble were captured in six binary liquids with a high-speed camera. We call the time between turning on the laser and bubble nucleation the delay time $\tau_{d}$. In Fig. 3.2 this delay time $\tau_{d}$ is shown as function of the mixing ratio for the six investigated binary liquids. As seen in Fig. 3.2 (a)-(c), $\tau_{d}$ decreases with increasing amount of the organic component in the three aqueous binary liquids (water/ethanol, water/acetone and water/1-propanol). Surprisingly, $\tau_{d}$ is roughly an order of magnitude smaller for purely organic binaries, see Fig. 3.2 (d)-(f). Moreover, for these it exhibits a different behavior, namely, $\tau_{d}$ varies only slightly with the mixing ratio for the ethanol/acetone and ethanol/2-propanol binary liquids, as shown in Fig. $3.2(\mathrm{~d}),(\mathrm{e})$. In contrast, $\tau_{d}$ increases with the amount of 1-butanol in the ethanol/1-butanol binary. Besides that, the laser power $P_{\ell}$ affects $\tau_{d}$. For all binary liquids $\tau_{d}$ decreases with increasing $P_{\ell}$, which has also been observed for pure water [30] and pure n-alkanes [31].

(a)

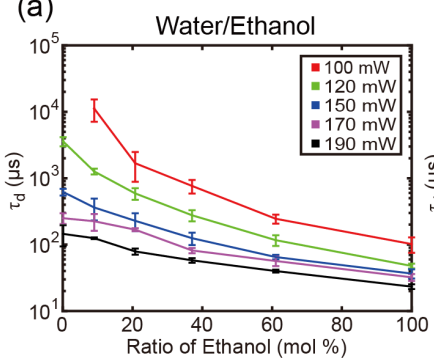

(d)

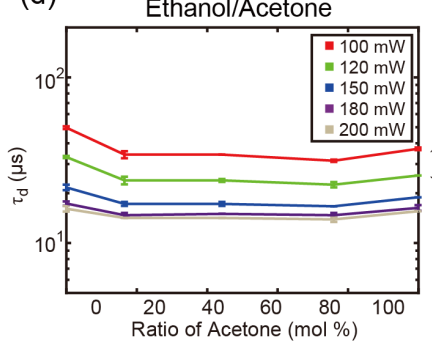

(b)

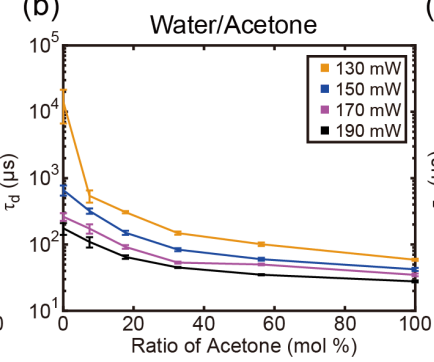

(e)

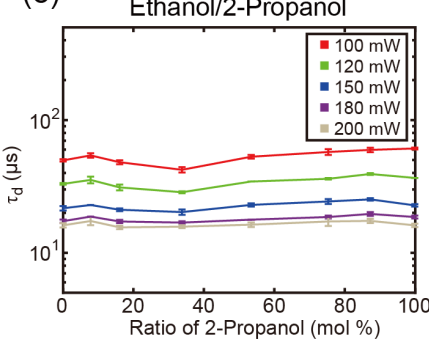

(c)

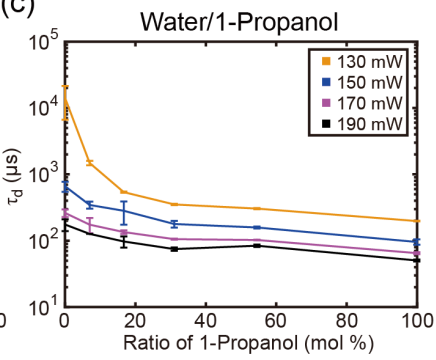

(f)

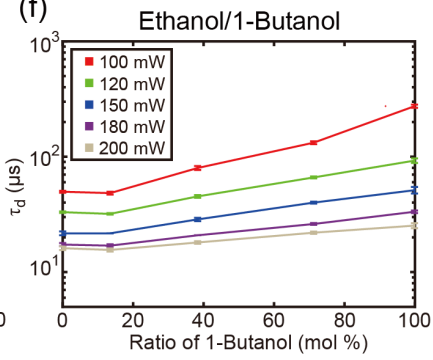

Figure 3.2: Delay time $\tau_{d}$ of the initial giant bubble as a function of the component ratio in the binary liquids water/ethanol (a), water/acetone(b), water/1propanol (c), ethanol/acetone (d), ethanol/2-propanol (e) and ethanol/1butanol (f). 
In order to understand how the mixing ratio affects $\tau_{d}$, the change in parameters must be taken into account. In Table 3.1 the parameters of the various pure liquids are listed. Only the surface tension, thermal conductivity and the amount of dissolved gas (implied by the combination of $\mathrm{N}_{2}$ and $\mathrm{O}_{2}$ solubilities) exhibit a large difference between water and the organic liquids. However, the thermal conductivity can be ruled out, because it would predict the same behavior in all organic binaries, which is not the case. While the surface tension varies drastically for small deviations from the pure liquids, the amount of dissolved gas changes almost linearly over the whole range of mixing ratios. This allows to distinguish between the effects of the two and we find the surface tension insufficient to explain the behavior of $\tau_{d}$. Our reasoning is elaborated in the Appendix. Consequently, the amount of dissolved gas should play a major role and we show $\tau_{d}$ as function of it in Fig. 3.3 (a). $\tau_{d}$ decreases with an increase in the dissolved gas for all shown liquids individually. Moreover, the global behavior also shows the decrease of $\tau_{d}$ with increasing amount of dissolved gas, which can be fitted with a power law $\tau_{d} \propto\left(V_{g} / V_{l}\right)^{-\gamma}$. We observe the same global behavior for all laser powers investigated, though the power law exponent $\gamma$ decreases with increasing $P_{\ell}$, see Fig. 3.3 (b). Hence, the amount of dissolved gas is the most important parameter for $\tau_{d}$. This is supported further by prior investigations of Wang et al. for pure water that showed an increased $\tau_{d}$ for degassed water [30] and also fits to the results on n-alkanes [31]. One might wonder whether it is the total amount of dissolved gas or the saturation level that causes this effect [30]. But our results demonstrate that it is the total amount of dissolved gas, because all binary liquids are saturated and the effect appears nonetheless.

In order to understand the mechanism by which the dissolved gas affects $\tau_{d}$, we study the nucleation temperature $T_{n}$. To determine $T_{n}$, we first study the temperature evolution around a single nanoparticle. We assume a spherical geometry and constant thermal properties and solve the spherical linear Fourier equation for heat conduction,

$$
\partial_{t}(T(r, t))=\frac{p_{\ell}(r, t)}{\rho c_{p}}+\kappa \frac{1}{r^{2}} \partial_{r}\left(r^{2} \partial_{r}(T(r, t))\right),
$$

where $\kappa, \rho$, and $c_{p}$ are thermal diffusivity, density, and heat capacity of the liquid, respectively, $r$ is the distance to the GNP, and $p_{\ell}(r, t)$ is the deposited power density (unit in $\mathrm{W} / \mathrm{m}^{3}$ ). Then, $T_{n}$ and the corresponding $\tau_{d}$ can be calculated by the superposition of GNPs. Further information on this calculation can be found in Wang et al. (2018) [30]. We fit the calculated $\tau_{d}$ with 

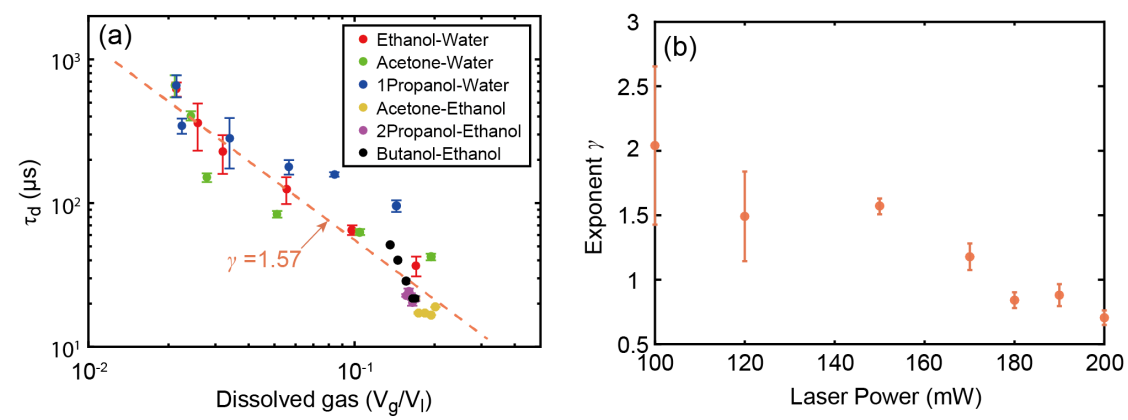

Figure 3.3: (a) Exemplary case of the delay time $\tau_{d}$ as a function of the dissolved gas $\left(\mathrm{N}_{2}+\mathrm{O}_{2}\right)$ in different binary liquids at $150 \mathrm{~mW}$. The dashed line represents a power law fit $\tau_{d} \propto\left(V_{g} / V_{l}\right)^{-\gamma}$. (b) Exponent $\gamma$ of the power law fit for the dissolved gas at various laser powers.

the experimental data in Fig. 3.2 to determine the corresponding $T_{n}$. This is exemplarily shown for pure water in Figs. 3.4 (a),(b), where the blue curve is the fit. It is located between two restrictions - namely, the boiling temperature ( $T_{\text {boil }}$, orange curve) and the spinodal temperature ( $T_{\text {spin }}$, green curve). The boiling, nucleation, and spinodal temperatures for the binary liquids at different component ratios are shown in Figs. 3.4 (d)-(i).

For aqueous binary liquids $T_{n}$ continuously decreases from $285^{\circ} \mathrm{C}$ to $180{ }^{\circ} \mathrm{C}$, $150^{\circ} \mathrm{C}$ and $185^{\circ} \mathrm{C}$ with an increasing amount of ethanol, acetone and 1propanol, respectively, see Figs. $3.4(\mathrm{~d})$-(f). In the ethanol/acetone and ethanol/2-propanol binary liquids, $T_{n}$ remains constant at approx. $170^{\circ} \mathrm{C}$ while varying the mixing ratio, as shown in Figs. $3.4(\mathrm{~g}),(\mathrm{h})$. Contrary, Fig. 3.4 (i) shows that $T_{n}$ slightly increases with an increase of the amount of 1butanol in the ethanol/1-butanol binary.

Now we can explain how the dissolved gas affects $\tau_{d}$. The nucleation temperature $T_{n}$ changes with the amount of dissolved gas, because dissolved gas molecules can act as nucleation sites [70, 91, 92]. Hence, an increase in the amount of dissolved gas reduces $T_{n}$. Therefore, the decrease in $T_{n}$ in the aqueous binaries with an increase of the organic component (see Figs. 3.4 (d)(f)) is a result of the increase in dissolved gas in the binaries. Moreover, this decrease in $T_{n}$ leads to faster nucleation and thereby explains the reduction in $\tau_{d}$ with increasing amount of dissolved gas (see Fig. 3.3(a)). The same holds true for the organic binaries. For the ethanol/1-butanol the amount of dissolved gas is slightly decreasing with increasing amount of 1-butanol and 


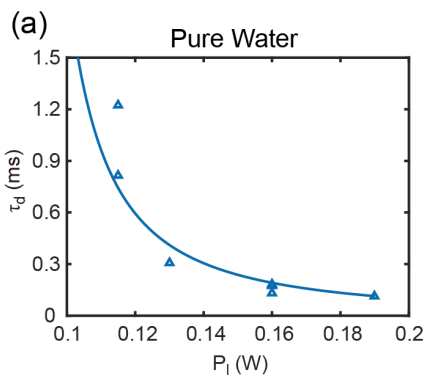

(d)

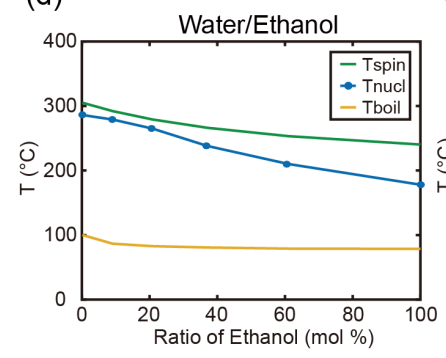

(g)

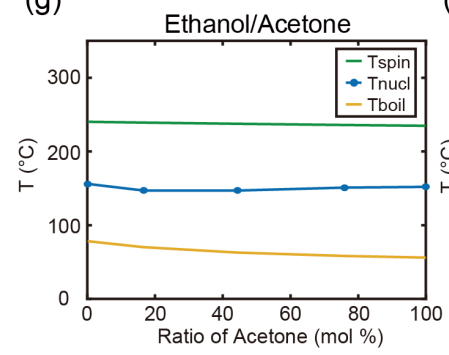

(b)

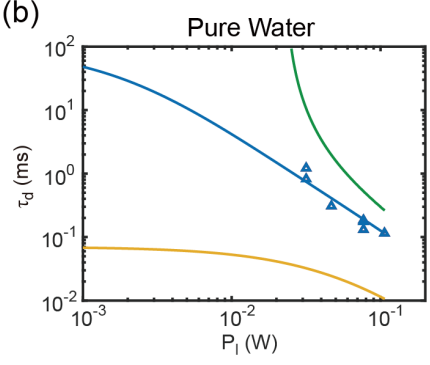

(e)

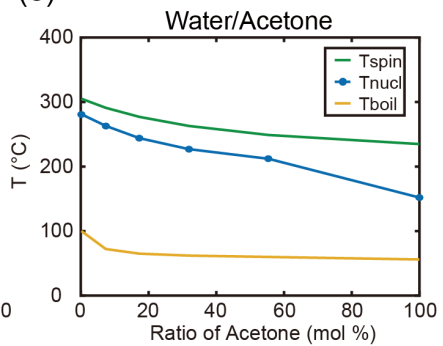

(h)

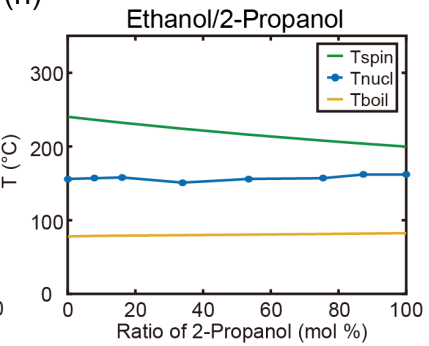

(c)

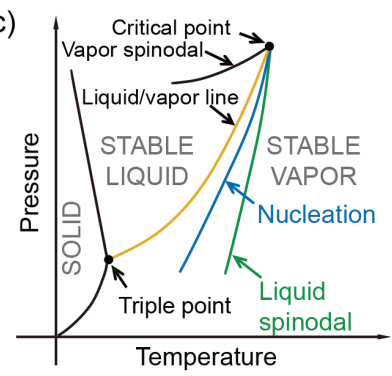

(f)

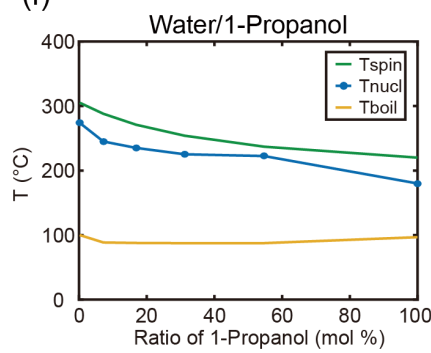

(i)

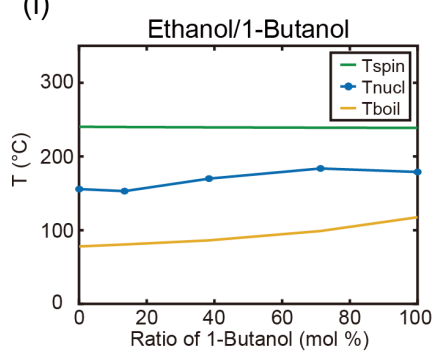

Figure 3.4: (a),(b) Delay time $\tau_{d}$ as a function of the laser power $P_{\ell}$ for pure water and the fit for the nucleation temperature (blue line) in a linear and double logarithmic plot. The green curves represent the spinodal temperature and the orange curves the boiling temperature. (c) Schematic phase diagram. The nucleation temperature of the initial giant bubble (blue) as a function of the mixing ratio in the binary liquids (d) water/ethanol, (e) water/acetone, (f) water/1-propanol, (g) ethanol/acetone, (h) ethanol/2propanol and (i) ethanol/butanol.

consequently a rise in $T_{n}$ and thereby also in $\tau_{d}$ is observed. The changes are not as large as for the aqueous binaries because the change in the amount of dissolved gas is smaller. The change is even smaller for the ethanol/acetone and ethanol/2-propanol binaries and therefore $T_{n}$ and $\tau_{d}$ remain almost con- 
stant.

What remains to be explained is how the laser power $P_{\ell}$ affects the behavior of $\tau_{d}$. The nucleation temperature $T_{n}$ is independent of $P_{\ell}$ [30]. Therefore, a higher $P_{\ell}$ only increases the heating rate and thereby reduces the time to reach $T_{n}$. Consequently, the nucleation happens earlier and $\tau_{d}$ decreases for higher laser powers as seen in Fig. 3.2. Moreover, this is also a possible explanation for the reduction of the exponent $\gamma$ for higher laser powers. If the temperature rises faster, the time difference between different $T_{n}$ 's decreases. Hence, the effect of the change in $T_{n}$ due to the dissolved gas on $\tau_{d}$ decreases and thereby also the exponent $\gamma$.

Besides the delay time, it is also important to understand how the volume of the bubble is determined. To compare the different binary liquids, we define the accumulated energy before nucleation as the deposited energy $E_{d}=\tau_{d} \cdot P_{\ell}$. In Fig. 3.5 the first column shows the bubble volume versus $E_{d}$ for various mixing ratios and exemplarily for the binaries water/ethanol (a), ethanol/acetone (d) and ethanol/1-butanol (g). For all cases we can identify a linear behavior between the volume and $E_{d}$ at low energies. However, the slope of this linear behavior varies with the mixing ratio, as shown in the second column of Fig. 3.5. While for the water/ethanol binary the slope remains constant at approx. $6 \mu \mathrm{m}^{3} / \mu \mathrm{J}$, for the ethanol/acetone binary it increases with increasing amount of acetone from $5.3 \mu \mathrm{m}^{3} / \mu \mathrm{J}$ to $8.7 \mu \mathrm{m}^{3} / \mu \mathrm{J}$. Moreover, for the ethanol/1-butanol binary the slope decreases from $5.3 \mu \mathrm{m}^{3} / \mu \mathrm{J}$ to $2.0 \mu \mathrm{m}^{3} / \mu \mathrm{J}$ with increasing amount of 1-butanol in the binary.

In order to explain this difference in behavior, we have to look at the energy balance. We assume that the vaporization energy per mole $E_{l-v}(\mathrm{~kJ} / \mathrm{mol})$ can be approximated by the energy needed to heat the liquid to the boiling temperature $T_{\text {boil }}$ and the latent heat of vaporization $H_{v a p}$.

$$
E_{l-v}=\int_{T_{0}}^{T_{b o i l}} c_{P, m} d T+H_{v a p}\left(T_{b o i l}\right) .
$$

The first term is the energy needed for the heating described as the integral over the molar heat capacity at constant pressure $c_{P, m}$ from ambient temperature $T_{0}$ to the boiling temperature $T_{\text {boil }}$. While this is easily calculated for pure liquids, mixtures are more complicated. Therefore, we estimate $E_{l-v}$ for the mixtures by the pure liquid values weighted by their mole fraction in the vapor. This is similar to how we estimated the liquid mixture parameters, however, the gas phase mole fractions are used instead to take into account that preferential evaporation of one species can occur. The mole fraction in the 
(a)

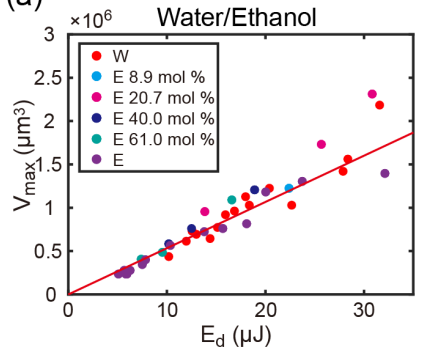

(d)

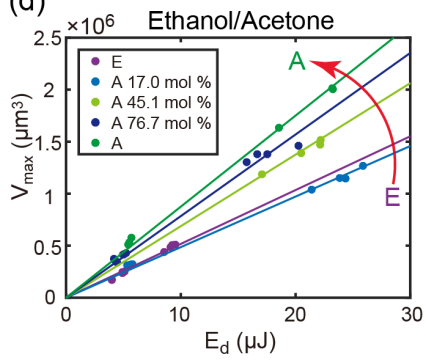

(g)

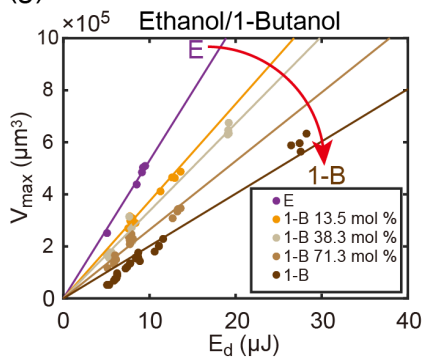

(b)

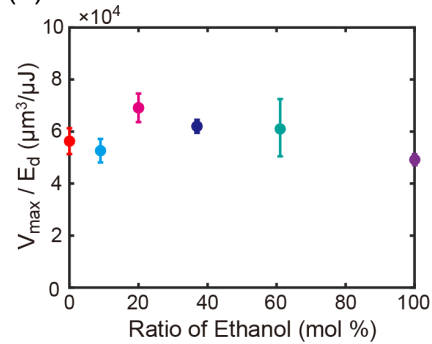

(e)

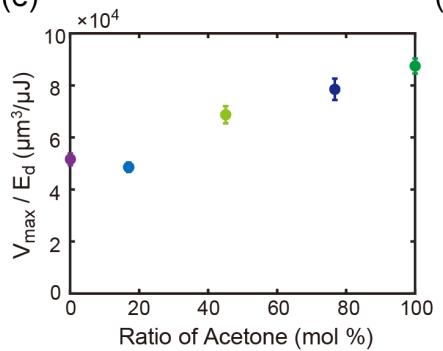

(h)

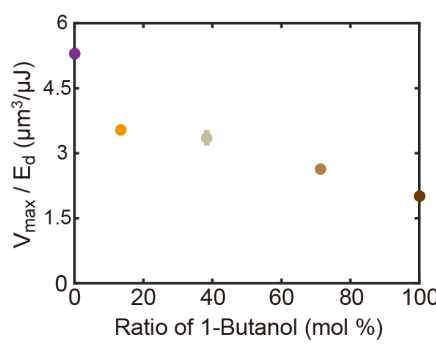

(c)

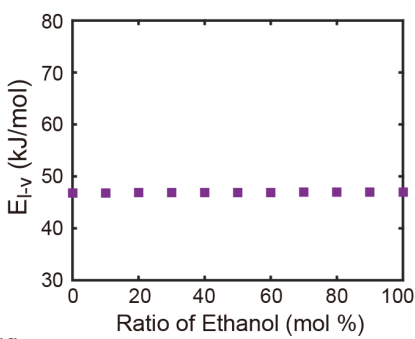

(f)

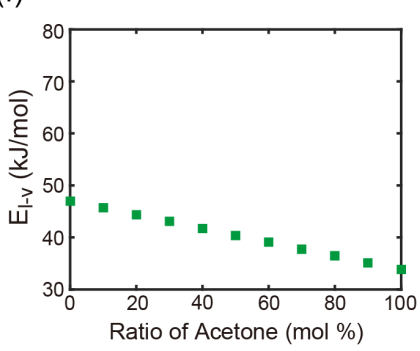

(i)

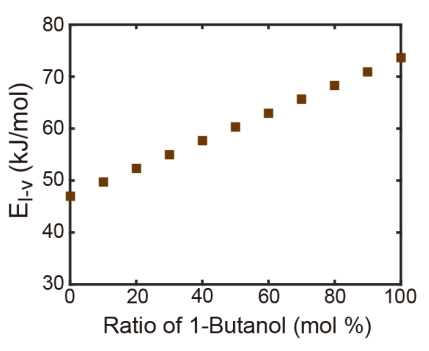

Figure 3.5: First column: Maximal bubble volume as a function of the deposited energy in the binaries ethanol/water (a), ethanol/acetone (d) and ethanol/1-butanol (g). Second column: Slope of the volume growth as a function of the mixing ratio for the binaries ethanol/water (b), ethanol/acetone (e) and ethanol/1-butanol (h). Third column: $E_{l-v}$ of the liquid as a function of its mixing ratio for the binaries ethanol/water (c), ethanol/acetone (f) and ethanol/1-butanol (i).

vapor can be determined by the mole fraction in the liquid using vapor-liquid equilibrium data [93]. Hence, we use for the mixtures

$$
E_{l-v}=\alpha \cdot E_{l-v, 1}+(1-\alpha) \cdot E_{l-v, 2}
$$

where $\alpha$ is the mole fraction in the vapor of the first binary component and 
$E_{l-v, 1}$ and $E_{l-v, 2}$ are the pure liquid values of component 1 and 2 respectively. If we assume that all the deposited energy is used to create vapor and the ideal gas law can be applied, we find the following relation between the deposited energy and the volume

$$
E_{d}=n \cdot E_{l-v}=\frac{P_{s a t} V}{R T} \cdot E_{l-v} .
$$

Here $R$ is the universal gas constant, $T$ the temperature, $P_{\text {sat }}$ the saturation pressure, $n$ the amount of vapor in mole and $V$ the bubble volume at maximal expansion. Consequently, we expect $V / E_{d}$ to be inversely proportional to $E_{l-v}$, see Eq. 3.5

$$
\frac{V}{E_{d}}=\frac{R T}{P_{s a t}} \cdot \frac{1}{E_{l-v}} .
$$

In the last column of Fig. 3.5 the behavior of $E_{l-v}$ as function of the mixing ratio is shown. While the slope remains constant for the ethanol/water binary, the same holds true for $E_{l-v}$ that stays at approx. $46.75 \mathrm{~kJ} / \mathrm{mol}$. For the increasing slope of the acetone/ethanol binary, $E_{l-v}$ decreases from $47 \mathrm{~kJ} / \mathrm{mol}$ to $33 \mathrm{~kJ} / \mathrm{mol}$. Finally, for the decreasing slope of the 1-butanol/ethanol binary, $E_{l-v}$ increases from $47 \mathrm{~kJ} / \mathrm{mol}$ to $64 \mathrm{~kJ} / \mathrm{mol}$. In comparison to the slope in the second column, the behavior is indeed inversely proportional to $E_{l-v}$, as Eq. 3.5 suggests.

To show the global behavior, the slopes are extracted from all liquids and plotted against the right term of Eq. 3.5 in Fig. 3.6. As expected from Eq. 3.5, we observe a linear increase for all liquids individually as well as globally, indicated by the dashed orange line. The only exception is the binary ethanol/2-propanol, which will be explained later. If we further analyze the global behavior, we find that the increase by an order of magnitude in $V / E_{d}$ cannot be explained by the change in $R T / P_{\text {sat }}$, since it only varies within $16 \%$ of the maximal value. Hence, the volume per energy is dominated by $E_{l-v}$.

The reason why the global behavior and the ethanol/2-propanol case are not perfectly linear is probably related to the efficiency (slope of Fig. 3.6). We assumed that the efficiency of the energy conversion from the absorbed light to the vapor bubble is the same for all liquids. In fact, this is not the case and the efficiency varies between $5 \%$ and $12 \%$. Therefore, the slope in Fig. 3.6 varies slightly for each liquid, which leads to the deviation from the ideal case. In which way and why the efficiency changes will be subject of another study. 


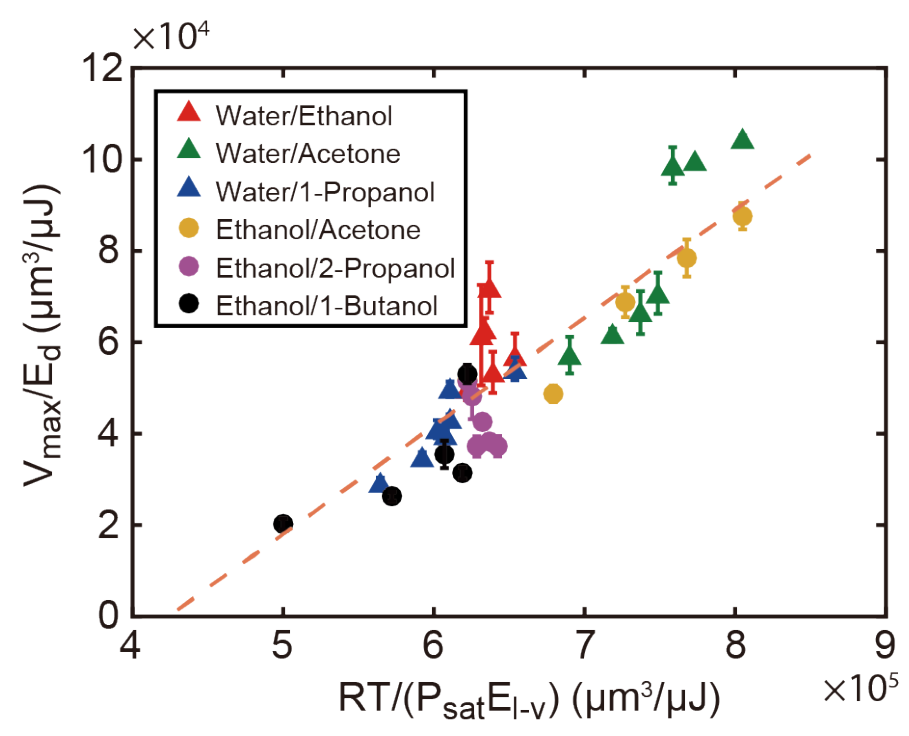

Figure 3.6: Maximal bubble volume per deposited energy as a function of $R T / P_{s a t} E_{l-v}$; the dashed line is a linear fit.

\subsection{Conclusions}

In summary, we have shown how the volume and delay time of the initial giant bubble are controlled by the liquid parameters. This delay time between the start of the laser heating and the bubble nucleation $\tau_{d}$ is determined by the absolute amount of dissolved gas. The gas molecules act as nucleation sites reducing the necessary nucleation temperature and thereby facilitate faster nucleation. The delay time can be tuned further by changing the laser power and consequently the heating ratio. Furthermore, we revealed that the volume of the bubble at a given energy is determined by the liquids energy needed for vaporization $E_{l-v}$. These results allow to tune the delay time and size of a vapor bubble by varying the corresponding liquid parameters. We envision that our findings will allow finer bubble control in applications which is particularly promising for medical applications. 


\subsection{Appendix}

\subsubsection{The effect of surface tension on nucleation}

Although in our experiments a clear separation between the effect of surface tension and dissolved gas is not feasible, strong arguments for the dominating effect of the dissolved gas are available. The main difference between surface tension and the amount of dissolved gas is the way they change with the mixing ratio. We will consider the water/1-propanol binary, because in this case the difference is particularly pronounced as shown in Fig. 3.7 (a).

a)

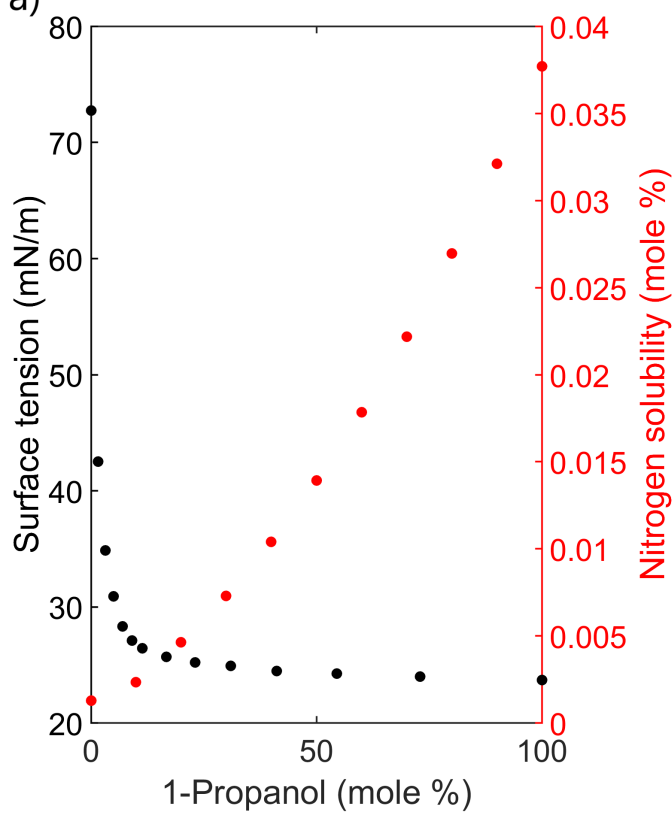

b)

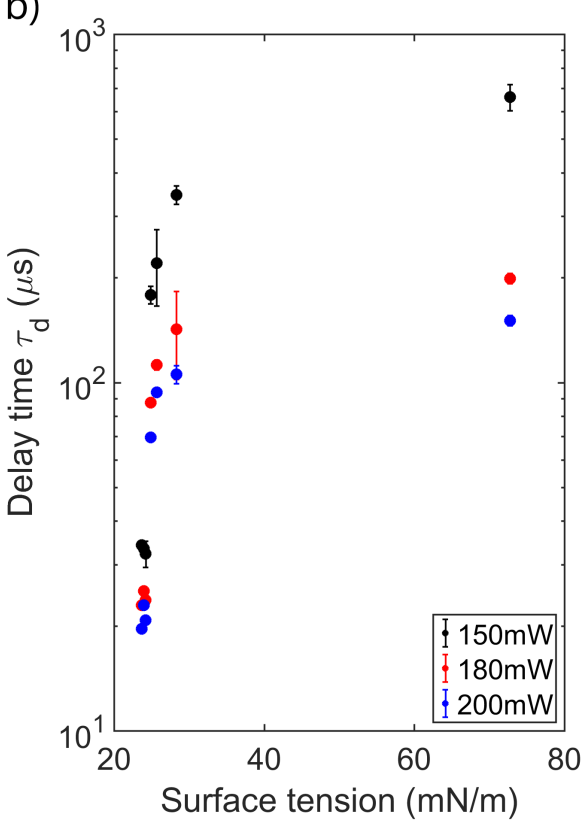

Figure 3.7: (a) Surface tension (black) and nitrogen solubility (red) as a function of the mixing ratio for the water/1-propanol binary liquid. Data taken from Vazquez et al. (1995) and Battino et al. (1984) [86,94]. (b) Delay time $\tau_{d}$ as a function of the surface tension of the same binary liquid for various lasers powers.

Initially, the surface tension drops sharply from $72.75 \mathrm{mN} / \mathrm{m}$ to 30.87 $\mathrm{mN} / \mathrm{m}$ by adding only $5.0 \%$ mole fraction of 1 -propanol. Afterwards, it decreases slowly to the pure 1-propanol value of $23.69 \mathrm{mN} / \mathrm{m}$ [94]. In contrast, the mole fraction of the dissolved gas increases almost linearly with the mix- 
ing ratio from pure water $\left(0.13 \cdot 10^{-4}\right)$ to pure 1-propanol $\left(3.77 \cdot 10^{-4}\right)[86]$. Now we can distinguish the effects of the two parameters, because the effect of surface tension will be confined to small deviations from the pure liquids and the dissolved gas will affect all mixing ratios. As shown in Fig. 3.7 (b) for the water/1-propanol binary liquid the delay time $\tau_{d}$ barely varies for the large change in surface tension from $72.75 \mathrm{mN} / \mathrm{m}$ to $28.31 \mathrm{mN} / \mathrm{m}$. In contrast, for the comparatively small change from $28.31 \mathrm{mN} / \mathrm{m}$ to $23.60 \mathrm{mN} / \mathrm{m}$ the delay time shortens by an order of magnitude. This indicates that the surface tension is not the dominant parameter for $\tau_{d}$. However, $\tau_{d}$ follows the trend of the dissolved gas well as shown in Fig. 3.3. Moreover, the experiments of Wang et al. (2018) with degassed water show that reducing only the amount of dissolved gas (without varying the surface tension) causes an increase in $\tau_{d}[30]$. In summary, the surface tension appears to be insufficient to explain the changes in $\tau_{d}$, whereas the dissolved gas allows to explain those even if no other parameter is varied. Hence, we conclude that the dissolved gas is the dominating parameter for $\tau_{d}$ and not the surface tension. 


\section{Chapter 4}

\section{Periodic bouncing of a plasmonic bubble in a binary liquid by competing solutal and thermal Marangoni forces 1}

The physicochemical hydrodynamics of bubbles and droplets out of equilibrium, in particular with phase transitions, displays surprisingly rich and often counterintuitive phenomena. Here we experimentally and theoretically study the nucleation and early evolution of plasmonic bubbles in a binary liquid consisting of water and ethanol. Remarkably, the submillimeter plasmonic bubble is found to be periodically attracted to and repelled from the nanoparticledecorated substrate, with frequencies of around a few $\mathrm{kHz}$. We identify the competition between solutal and thermal Marangoni forces as origin of the periodic bouncing. The former arises due to the selective vaporization of ethanol at the substrate's side of the bubble, leading to a solutal Marangoni flow towards the hot substrate, which pushes the bubble away. The latter arises due to the temperature gradient across the bubble, leading to a thermal Marangoni

\footnotetext{
${ }^{1}$ Published as: Binglin Zeng, Kai Leong Chong, Yuliang Wang, Christian Diddens, Xiaolai Lia, Marvin Detert, Harold J. W. Zandvliet, and Detlef Lohse, Periodic bouncing of a plasmonic bubble in a binary liquid by competing solutal and thermal Marangoni forces, Proc. Natl. Acad. Sci. U. S. A. 118, e2103215118 (2021). Experimental work is done by Zeng. Theoretical work is done by Lohse and Chong.
} 
flow away from the substrate which sucks the bubble towards it. We study the dependence of the frequency of the bouncing phenomenon from the control parameters of the system, namely the ethanol fraction and the laser power for the plasmonic heating. Our findings can be generalized to boiling and electrolytically or catalytically generated bubbles in multicomponent liquids. 


\subsection{Introduction}

Bubbles and bubble nucleation are ubiquitous in nature and technology, e.g. in boiling, electrolysis, and catalysis, where the phenomena connected with them have tremendous relevance for energy conversion, or in flotation, sonochemistry, cavitation, ultrasonic cleaning, and biomedical applications of ultrasound and bubbles. This also includes plasmonic bubbles, i.e., bubbles nucleating at liquid-immersed metal nanoparticles under laser irradiation, due to which an enormous amount of heat is produced thanks to a surface plasmon resonance [28, 30, 51, 77, 81]. For an overview on the fundamentals of bubbles and their applications we refer to our recent review article [95]. In general, in these applications the bubble nucleation does not occur in a pure liquid, but in multicomponent liquids. Because of that, various additional forces and effects come into play [14], which are not relevant in pure liquids. Examples are the Soret effect [15, 96, 97] or body forces arising due to density gradients. Once the multicomponent systems have interfaces, solutal Marangoni forces become relevant. The phenomena become even richer once phase transitions occur in such systems, e.g. solidification [98, 99, evaporation [17, 18, 100 105] or dissolution of a multicomponent droplets [106 109], or nucleation of a new phase such as in the so-called ouzo effect [19,20] or in boiling [110], electrolysis [111, 112], or catalysis [113,114]. Similarly, also chemical reactions occurring at the interface in a multicomponent liquid lead to spectacular effects, such as swimming droplets [115, 116], phoretic self-propulsion [117-120] or pattern formation in electroconvection [121]. The whole field could be summarized as physicochemical hydrodynamics, and though this is a classical subject [122], it got increasing attention in recent years due to its relevance for various applications, due to new experimental and numerical possibilities, and due to the beauty of the often surprising and counterintuitive phenomena. For recent reviews on physicochemical hydrodynamics we refer to references [123, 124.

To exactly analyse the various competing forces playing a role in physicochemical hydrodynamical systems, one has to strive to have simple and clean geometries, allowing for precise measurements and a theoretical and numerical approach. For example, in ref. [125] we analysed the competition between solutal Marangoni forces, gravity, and thermal diffusion by studying an oil droplet in a stably stratified liquid consisting of ethanol and water, imposing density and surface tension gradients on the droplet. Depending on the control parameters, the droplet was either stably levitating or jumping up and down, with a very low frequency of $\sim 0.02 \mathrm{~Hz}$. Similar droplet and bubble 
oscillations originating from the competition between solutal Marangoni forces and gravity were observed in ref. [126].

In this paper, we will report and analyse another controlled physicochemical hydrodynamic bouncing phenomenon, even involving phase transitions, namely that of a nucleating plasmonic bubble [30, 51, 77], but now in an initially homogeneous binary liquid, for which the delay of bubble nucleation after turning on the laser depends on the composition of the binary liquid and the amount of dissolved gas [127], (next, of course, to the power of the employed laser). As in ref. [125], we will again see a bouncing behavior, but this time on a much faster timescale, corresponding to frequencies of $\sim 10^{3}$ $\mathrm{Hz}$. We will use this controlled physicochemical hydrodynamic system out of equilibrium to probe the competition between solutal and thermal Marangoni forces. That, in the presence of concentration gradients, the latter can compete with the former ones, is only possible thanks to the very high temperature gradients in the system of a nucleating plasmonic bubble. Under more standard conditions, such as for the evaporation of a binary droplet, the solutal Marangoni forces tend to be much stronger than the thermal ones [100].

We note that plasmonic bubbles are in itself very interesting with potential applications in biomedical diagnosis and therapy, micro- and nanomanipulation, and catalysis [54, 81, 128, 129]. Also note that plasmonic bubbles directly after nucleation are pure vapor bubbles [130] originating from evaporation of the surrounding liquid, but during their expansion they are invaded by dissolved gas from the surrounding liquid [32, 33, 62, 127], which in the long term crucially determines their dynamics and lifetime.

The key idea of this study here will build on the selective heating of the liquid surrounding the plasmonic bubble, namely on the side of the plasmonic nanoparticles. This leads to very strong temperature gradients across the bubble and thus to thermal Marangoni forces and at the same time to strong concentration gradients, as the evaporation of the surrounding binary liquid is selective, favoring the liquid with the lower boiling point. Thus also solutal Marangoni forces along the bubble-liquid interface emerge. As we will see, which of these two different Marangoni forces is stronger depends on time and bubble position, leading to an oscillatory or bouncing bubble behavior.

\subsection{Experimental setup and findings}

The experimental setup is as follows: The gold nanoparticle decorated substrate was put in a quartz glass cuvette $(10 \times 10 \times 45 \mathrm{~mm})$ filled with an ethanol- 
water binary liquid of varying composition in Appendix for more details on sample preparation). A continuous laser (Cobolt Samba) of $532 \mathrm{~nm}$ wavelength was used for substrate irradiation from the bottom side. The laser power was controlled by using a half-wave plate and a polarizer and measured by a photodiode power sensor (S130C, ThorLabs). Two high speed cameras were installed in the setup to monitor the dynamics of the generated bubbles. One (Photron SA7) was equipped with a $5 \times$ long working distance objective (LMPLFLN, Olympus) for bottom view imaging, and the other one (Photron SAZ) with a $10 \times$ long working distance objectives and operated at $200 \mathrm{kfps}$ for fast imaging.

A series of typical snapshots of the bubble motion in an ethanol-water binary liquid (ethanol: $75 \mathrm{wt} \%$ ) for two early bouncing cycles (about $19 \mathrm{~ms}$ after nucleation of the plasmonic bubble) is shown in Fig. 4.1(a). The radius $R(t)$, the distance of the bubble center from the plasmonic-particle-decorated substrate $H_{C}(t)$, and the distance between the bubble bottom and the substrate $H_{B}(t)$ are plotted in Figs. 4.1(b)-(d), with two different temporal magnifications.

In the first three frames of Fig. 4.1(a), (1)-(3), the bubble is moving towards the substrate and expanding under laser irradiation. At $370 \mu \mathrm{s}$, the bubble bottom touches the substrate and the bubble radius reaches a maximum value of $24 \mu \mathrm{m}$. Subsequently, the bubble jumps up and at the same time shrinks ((3)-(5)). At $870 \mu \mathrm{s}$, the bubble radius has its minimum of $17 \mu \mathrm{m}$ and the distance between its bottom and substrate reaches $22 \mu \mathrm{m}((6))$. After that, it moves again towards the substrate for another cycle ((6)-(10)). In the entire process, the bubble continuously bounces towards and away from the substrate for 34 times. Finally, it remains sitting on the substrate and enters its stable and monotonous growth phase.

To understand the origin of the bouncing behavior, we investigate the flow dynamics by particle image velocimetry (PIV). Fluorescent particles with a diameter of $1.1 \mu \mathrm{m}$ and a concentration of $100 \mu \mathrm{g} / \mathrm{mL}$ were added to the ethanol-water binary liquid, and illuminated with a laser (see Appendix for more details and results). The results of the PIV measurements for one jumping cycle are shown in Fig. 4.2(a). In Fig. 4.2(b) we also show the measured time dependence of the tangential and the normal velocity components on the surface of the bubble on the side towards the heated substrate (see sketch in Fig. 4.2(a)-II), see also Fig. 4.4 in the Appendix. Flow illustrations during the repelling and attracting phase are shown in the left and right panel of Fig. 4.2. (c), respectively. 

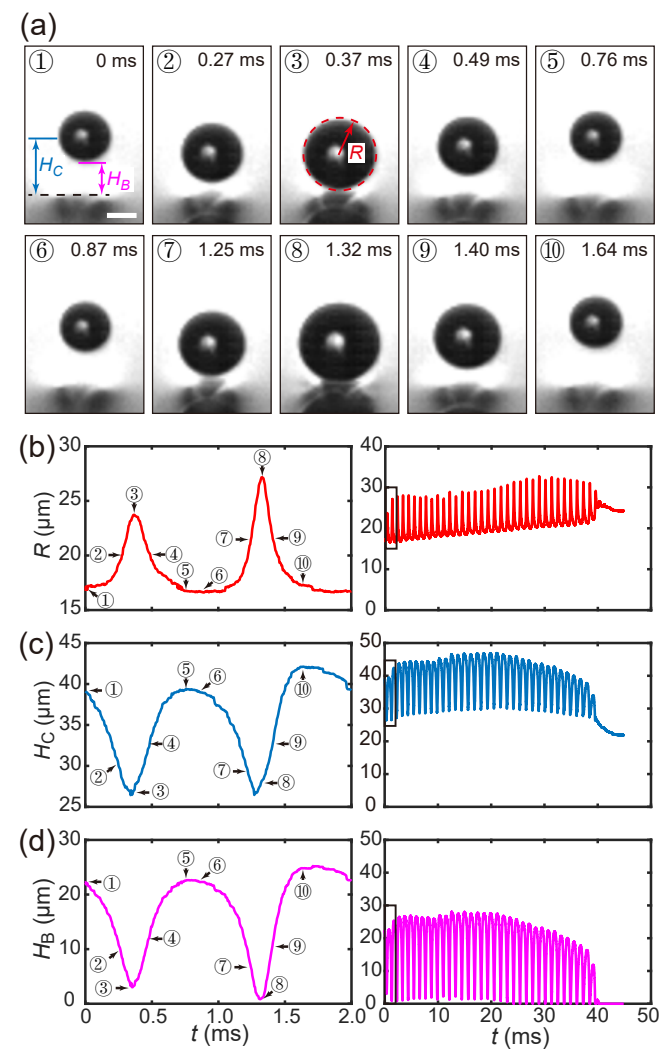

Figure 4.1: Successive bouncing of a plasmonic bubble in an ethanol-water binary liquid with ethanol concentration at $f_{e}=75 \mathrm{wt} \%$ and laser power $P_{\ell}=50 \mathrm{~mW}$ under continuous laser irradiation. (a) Experimental snapshots of the bubble bouncing for the first two cycles. The scale bar is $20 \mu \mathrm{m}$. The instantaneous bubble radius $R$, the center position $H_{C}$, and distance $H_{B}$ from the bottom of the bubble to the substrate with plasmonic particles are defined in the 3rd and 1st image, respectively. Obviously, $H_{C}=H_{B}+R$. (b-d) Left: The bubble radius $R$, the center position $H_{C}$, and the distance $H_{B}$ from the bottom versus time $t$ for the first two cycles of the bouncing process. Right: The same on a much longer time scale. The area selected by the rectangular boxes is the time interval shown in the left figures. This particular bubble bounces 34 times within $40 \mathrm{~ms}$, corresponding to a mean bouncing frequency of $f_{b}=0.85 \mathrm{kHz}$. 
(a)

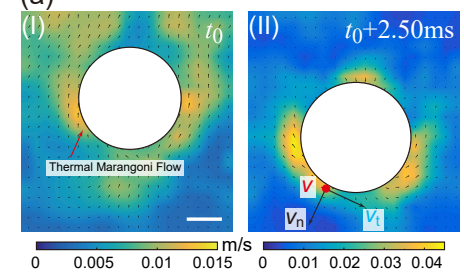

(b)
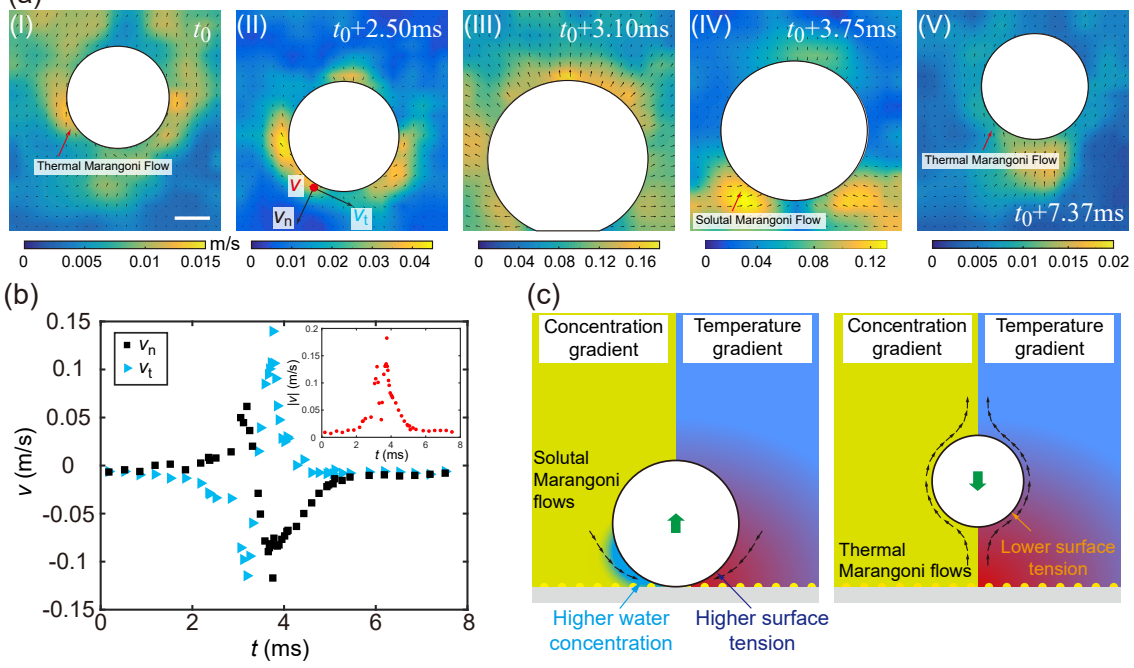

(c)

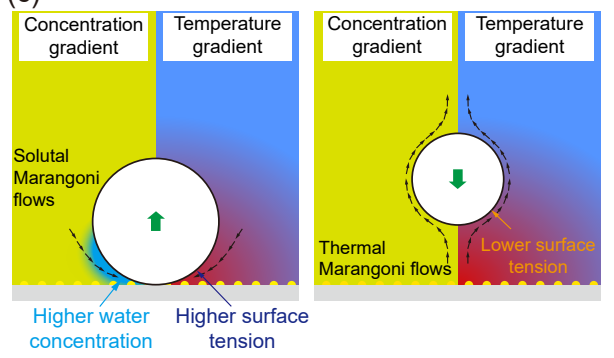

Figure 4.2: (a) PIV measurements of the plasmonic bubble for one bouncing cycle. The scale bar is $50 \mu \mathrm{m}$. In the experiment, the control parameters (initial concentrations in the binary mixture) are the same as in Fig. 4.1. (I) The temperature gradient generates thermal Marangoni flow, which drives the bubble downwards. (II) Shortly after the bubble starts to move downwards, the thermal induced Marangoni flow becomes stronger. (III) The bubble hits the superheated surface, leading to vaporization of (mainly) ethanol near the substrate into the bubble and corresponding bubble growth. The bubble expanding flow can be recognized from the velocity vectors pointing radially outwards from the bubble's center. (IV) The selective vaporization of ethanol from the binary liquid leads to a water rich area near the substrate, inducing a solutal Marangoni flows towards the bubble's bottom and correspondingly an upwards push of the bubble. (V) The Marangoni flow is dominated by the temperature gradient again when the bubble no longer feels a concentration gradient. In this cooler region it shrinks again. Note that there is a sideways motion of the bubble in subfigure $\mathrm{V}$ owing to the asymmetry in the solutal Marangoni flow (see subfigure IV). (b) Measured velocities as a function of time $t$ from the PIV measurement shown in (a). The given values refer to the positions shown in the subfigure in the lower right corner of Fig. 4.2(a)-II: $V_{n}$ (black) and $V_{t}$ (blue) are the normal and the tangential velocity component, respectively. The subfigure in the upper right corner displays the velocity magnitude $|V|=\sqrt{V_{n}^{2}+V_{t}^{2}}$ as function of time $t$. (c) Schematic diagrams of the bubble bouncing mechanism. The left figure sketches the repelling phase when the solutal Marangoni force dominates and the right one the attracting phase when the thermal Marangoni flow dominates. 


\subsection{Interpretation of the findings}

Our interpretation of the measurements is as follows: we start the cycle at moment (3) (Fig. 4.1(a)), when the bubble has its maximal extension and is (nearly) in touch (the minimum distance between the bubble and the wall is in range from 0 to $3 \mu \mathrm{m}$, depending on the values of the control parameters) with the plasmonic-particle-decorated and thus hot substrate. Correspondingly, on the bubble side towards the hot substrate there is selective evaporation of ethanol, due to the lower boiling point of ethanol as compared to water (Fig. 4.5 in the Appendix). This leads to a higher relative water concentration at that side of the bubble and thus to a solutal Marangoni flow along the bubble interface towards the substrate (Fig. 4.2(c) left), see Fig. 4.2(a). This flow pushes the bubble up, away from the substrate. As there the temperature is lower, the vapor inside the bubble partially condenses, preferentially at the side away from the substrate, and the bubble shrinks and experiences a decreasing concentration gradient along its interface and thus a weaker Marangoni force. At moment (6) the plasmonic bubble has reached its minimal size. However, it still experiences a strong temperature gradient as the thermal diffusivity is much faster than the solutal diffusivity. The ratio of the two diffusivities is the Lewis number $L e$, which is about 110 for ethanol at the temperatures we consider here. As a result, the thermal boundary layer is much thicker than the concentration boundary layer. The strong temperature gradient across the bubble with higher temperature (and thus lower surface tension) on the bubble side towards the substrate and lower temperature and thus higher surface tension on the opposite side leads to a thermal Marangoni flow along the bubble interface, away from the substrate. This flow pushes the bubble towards the substrate (moment (7) in Fig. 4.1(a) and right panel of Fig. 4.2 (c)) where it arrives at moment (8), which is equivalent to the starting moment (3) of the cycle.

We note here that in the bubble bouncing process gravity does not play any role, in contrast to the bubble bouncing experiment of ref. [125] that is done on much larger length scales. This can easily be seen from calculating the ratio $\Delta \rho g R^{2} / \Delta \sigma$ between gravity and the Marangoni force [124]. For a bubble with radius $R=2 \times 10^{-5} \mathrm{~m}$, a density of $\rho \approx 900 \mathrm{~kg} / \mathrm{m}^{3}$ for the water-ethanol mixture and $\Delta \sigma \approx 0.03 \mathrm{~N} / \mathrm{m}$ as approximate difference in surface tension between water and ethanol we obtain $\approx 10^{-4}$ for this ratio, i.e., negligible gravity. To further confirm that gravity does not play any role during this process, the sample substrate was placed upside down on the top window of the cuvette 
(a)

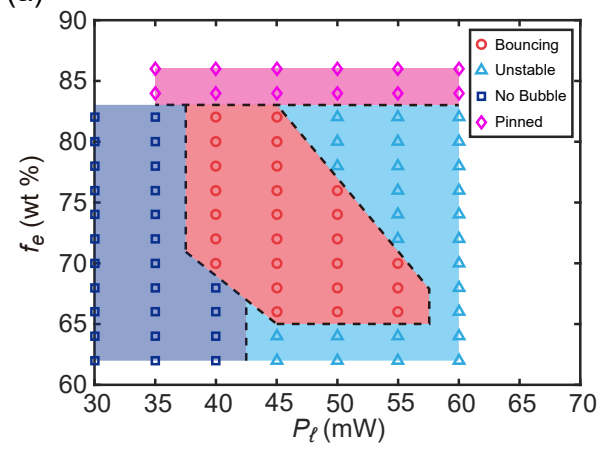

(b)

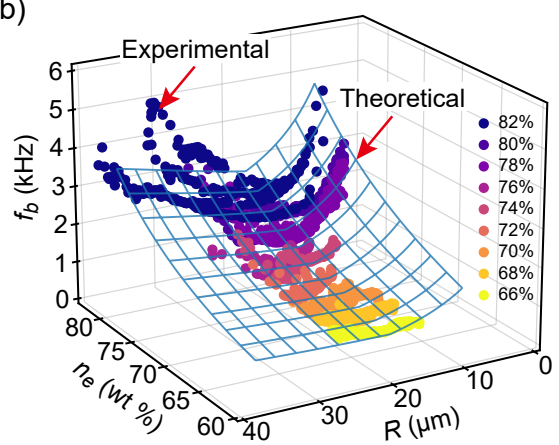

Figure 4.3: (a) Phase diagram for the bubble behaviors at the gold nanoparticle decorated sample surface upon continuous wave laser irradiation. The control parameters are the ethanol concentration $f_{e}$ and the laser power $P_{\ell}$. Bubble bouncing takes place in the red regime where $40 \mathrm{~mW}<P_{\ell}<55 \mathrm{~mW}$ and $66 \mathrm{wt} \%<f_{e}<82 \mathrm{wt} \%$. (b) Experimentally measured (data points, $P_{\ell}=45 \mathrm{~mW}$ ) and theoretically estimated (transparent curved surface) bubble bouncing frequency $f_{b}$ as function of the ethanol concentration $f_{e}$ and the bubble radius $R$. Both exhibit similar trends. For the theoretical curve the prefactor $k=23$ of the Prandtl-Blasius-Pohlhausen relation has been adapted to the experimental results.

and a bubble was produced below the sample substrate. The same bouncing phenomenon is observed.

\subsection{Parameter space}

To get more insight into the competition of the solutal and thermal Marangoni forces during the bouncing process, systematic experiments were conducted by tuning the laser power $P_{\ell}$ from $30 \mathrm{~mW}$ to $60 \mathrm{~mW}$ with ethanol concentrations $f_{e}$ from $62 \mathrm{wt} \%$ to $86 \mathrm{wt} \%$. In the phase diagram shown in Fig. 4.3(a), only in the region filled by red circles $\left(40 \mathrm{~mW}<P_{\ell}<55 \mathrm{~mW}\right.$ and 66 wt $\%<f_{e}<$ 82 wt \%) bubble bouncing takes place. Beyond this parameter range, there are three more phases in the phase diagram: unstable bubbles, no bubbles and pinned bubbles, respectively. For bouncing bubbles, we find that the bouncing frequency $f_{b}$ is strongly influenced by the ethanol concentration $f_{e}$ and the bubble radius $R$, as shown in Fig. 4.3 (b). It is found that $f_{b}$ increases 
monotonously with increasing $f_{e}$, while it depends non-monotonously on $R$. For $R \lesssim 15 \mu \mathrm{m}, f_{b}$ decreases with increasing $R$, while for $R \gtrsim 15 \mu \mathrm{m}, f_{b}$ increases with increasing $R$.

\subsection{Theoretical model}

To understand the observed dependence of $f_{b}$ on $f_{e}$ and $R$, we propose a simple theoretical model of the bouncing process. The essence of the competition between the solutal and the thermal forces acting on the bouncing plasmonic bubbles can be described by a simple effective point force ODE model for the bubble's center position $z=H_{c}(t)$ and its radius $R(t)$, with varying added mass of the bubble plus fluid acceleration, in the spirit of refs. [131 134], see also section IX of ref. [95]. Within such a model the normal (to the substrate) force on the moving bubble can in principle be described by [131]

$$
\begin{gathered}
F(t)=-4 \pi \rho \nu R(t) \dot{z}(t)+\frac{4}{3} \pi \rho R(t)^{3} \ddot{z}(t)+\frac{2}{3} \pi \rho\left(\frac{d\left[R(t)^{3} \dot{z}(t)\right]}{d t}+2 R(t)^{3} \ddot{z}(t)\right) \\
+8 \pi \rho \nu \int_{0}^{t} \exp \left[9 \nu \int_{\tau}^{t} R\left(t^{\prime}\right)^{-2} d t^{\prime}\right] \times \operatorname{erfc}\left[\sqrt{9 \nu \int_{\tau}^{t} R\left(t^{\prime}\right)^{-2} d t^{\prime}}\right] \frac{d[R(\tau) \dot{z}(\tau)]}{d \tau} d \tau \\
+F_{s-M}(R(t), z(t))+F_{t h-M}(R(t), z(t)) .
\end{gathered}
$$

Here it has been assumed that the bubble dynamics is not affected by wall effects, i.e., the prefactors of the various force contributions refer to the case of a bubble in the bulk.

The first term is the Stokes drag force on the bubble; taking the Stokes drag is justified as the bubble's translational Reynolds number $R e_{t}(t)=$ $\dot{H}_{c}(t) R(t) / \nu$ remains smaller than 1 . (In fact, an upper estimate with $\dot{H}_{c} \approx$ $0.06 \mathrm{~m} / \mathrm{s}, R \approx 2 \times 10^{-5} \mathrm{~m}$, and $\nu=2.2 \times 10^{-6} \mathrm{~m}^{2} / \mathrm{s}$ for the kinematic viscosity of the liquid gives $R e_{t}=0.54$ ). The other terms in the first row reflect added mass plus liquid acceleration; the term in second row reflects the Basset history force, and the terms in the last row are the solutal and thermal Marangoni forces, respectively. If the bubble is fully immersed in the boundary layers, the two Marangoni forces can be modeled as $F_{s-M} \sim+\gamma_{s} R^{2}$ and $F_{t h-M} \sim-\gamma_{t h} R^{2}$ [124, 135], where $\gamma_{s}$ and $\gamma_{t h}$ are the surface tension gradients caused by concentration difference and temperature difference along the vertical direction, respectively. Both are assumed to be constant within the respective boundary layers.

The concentration boundary layer thickness $\lambda_{s}$ is much smaller than the thermal boundary layer thickness $\lambda_{t h}$. It even holds $\lambda_{s}<R$ so that the 
solutal Marangoni force is relevant only near the substrate. This also becomes evident from the PIV experiments, see Fig. 4.2(a)-IV. Therefore, we consider the solutal Marangoni force on the bubble as a short impulse, i.e., like shooting a bullet. Correspondingly, we call our simple model the "bullet model". After the short impulse, the bubble's motion is dominated by the thermal Marangoni force $\sim-\gamma_{t h} R^{2}$ and the Stokes drag force $-4 \pi \rho \nu R \dot{z}(t)$. Accordingly, the dynamical equation for the bouncing bubble can be simplified to

$$
\frac{10}{3} \pi \rho R^{3} \ddot{z}(t)=-4 \pi \rho \nu R \dot{z}(t)-\gamma_{t h} R^{2} .
$$

Here we took the bubble radius as constant for simplicity, which is reasonable since the radius only considerably changes within a small fraction of time ( $15 \%$ ) during the bouncing cycle, see Fig. 4.7 of the Appendix. The prefactors of the first two terms in Eq. 4.2 again refer to the case of a bubble in the bulk and ignore possible modifications of the added mass coefficient and the drag coefficient due to wall effects, whereas for the third term we put the prefactor to 1 . Both is justified as later an unknown prefactor has to be introduced anyhow, in which such prefactors can be absorbed, see Eq. 4.5 below.

The initial condition for the position is $z(0)=0$ and the initial velocity $\dot{z}(0)$ follows from equating the work done by the solutal Marangoni force $W_{s-M}=$ $\int F_{s-M}(z) d z$ to the initial kinetic energy $E_{k}=\frac{1}{2}\left(\frac{10}{3} \pi \rho R^{3}\right) \dot{z}(0)^{2}$ for the moving liquid around the bubble. As $\lambda_{s}<R$, the solutal Marangoni force acts only in the concentration boundary layer and the work it performs can be estimated as $W_{s-M}=\gamma_{s} \lambda_{s}^{3}$. With these initial conditions for $z(0)$ and $\dot{z}(0)$, Eq. 4.2 can be solved analytically to finally arrive at an implicit equation for the duration time $t_{t u r}$ for the bubble to return to its initial position (which is the inverse bouncing frequency $f_{b}$ )

$$
t_{d u r}=f_{b}^{-1}=\frac{5 R^{2}}{6 \nu}\left(1+\sqrt{\frac{48 \pi}{5} \frac{\gamma_{s}}{\gamma_{t h}} \frac{\rho \nu^{2}}{\gamma_{t h} R^{2}} \frac{\lambda_{s}^{3}}{R^{3}}}\right)\left(1-e^{-\frac{6 \nu}{5 R^{2}} t_{d u r}}\right) .
$$

The thickness $\lambda_{s}$ of the concentration boundary layer is determined by the diffusion equation for a scalar field [136] as $\lambda_{s} \sim k \min \left(R, R_{\ell}\right) / \sqrt{S c}$, where $S c$ is the Schmidt number $S c=\nu / D$, with $D \approx 10^{-9} \mathrm{~m}^{2} / \mathrm{s}$ the mass diffusivity of water in ethanol. The expression for $\lambda_{s}$ takes into account that, if the bubble becomes larger than the radius $R_{\ell} \approx 20 \mu \mathrm{m}$ of the heated laser spot, the relevant length scale must be the concentration boundary layer thickness around that laser spot. The prefactor $k$ is a priori unknown and is adapted to the experimental data, see Fig. 4.3(b). With $k=23$ they can be well 
described. More details on the bullet model are given in the Appendix, see in particular Section 4.7.5 and Fig. 4.8.

It is instructive to identify the appropriate dimensionless control parameters of the bouncing plasmonic bubble. These are the solutal and thermal Marangoni numbers $M a_{s} \equiv R^{2} \gamma_{s} /(\rho \nu D)$ and $M a_{t h} \equiv R^{2} \gamma_{t h} /(\rho \nu D)$, respectively, the Schmidt number $S c$, and the ratio $R_{\ell} / R$ of the laser spot radius and the bubble radius. For the definition of the Marangoni numbers, we define the surface tension gradients $\gamma_{s}$ and $\gamma_{t h}$ separately for the concentration and the temperature differences within the respective boundary layers. This can reasonably be done as the solutal boundary layer thickness is much thinner than thermal one. With these definitions and using the viscous time scale $R^{2} / \nu$ and the bubble radius $R$ as reference scales, Eqs. 4.2 and 4.3 can be rewritten as

$$
\begin{gathered}
\frac{10}{3} \pi \tilde{z}^{\prime \prime}=-4 \pi \tilde{z}^{\prime}-\frac{M a_{t h}}{S c} \\
\tilde{t}_{d u r}=\tilde{f}_{b}^{-1}=\frac{5}{6}\left(1+\sqrt{k^{3} \frac{48 \pi}{5} \frac{M a_{s}}{M a_{t h}^{2} S c^{1 / 2}} \min \left(1, \frac{R_{\ell}}{R}\right)}\right)\left(1-e^{-6 \tilde{t}_{d u r} / 5}\right),
\end{gathered}
$$

reflecting that the bubble bouncing period indeed only depends on the four dimensionless groups $M a_{t h}, M a_{s}, S c$, and $R_{\ell} / R$, and that thermal and solutal Marangoni forces compete with each other. It also shows that there is only one free dimensionless parameter, $k$, in which possibly different prefactors in the terms of Eq. 4.2 can all be absorbed.

The bullet model allows us to understand the physics of the experimentally found parameter dependences of Fig. 4.3(b). First, the monotonous increase of the bouncing frequency $f_{b}$ on the ethanol concentration $f_{e}$ is due to the fact that, in the experimental parameter space of $62 \mathrm{wt} \%<f_{e}<86 \mathrm{wt} \%$, a higher $f_{e}$ leads to a smaller surface tension gradient $\gamma_{s}$. This results in a lower kinetic energy $E_{k}$ after the solutal Marangoni impulse, and thus to a higher bouncing frequency $f_{b}$ as the bubble is pulled back earlier by the thermal Marangoni force. Next, the nonmonotonous dependence of the bouncing frequency $f_{b}$ on the bubble radius $R$ is due to the transition of the concentration boundary layer thickness $\lambda_{s}$ from being determined by the bubble radius $R$ to being determined by the laser spot radius $R_{\ell}$. For a tiny bubble with $R<R_{\ell}$, an increase of $R$ results in an increase of the kinetic energy $\left(E_{k} \sim \gamma_{s} \lambda_{s}^{3}\right)$, leading to a decrease of $f_{b}$. In contrast, for $R \geq R_{\ell}$, the boundary layer thickness $\lambda_{s}$ is limited by $R_{\ell}$ and $E_{k} \sim \gamma_{s} \lambda_{s}^{3}$ remains nearly constant. However, the added mass of the bubble does increase with increasing $R$. This leads to a decrease 
of the initial velocity $\dot{z}(0)$ and an increase in the bouncing frequency $f_{b}$.

Finally, we put the experimentally found phase diagram of Fig. 4.3(a) showing that bubble bouncing can only take place if both the laser power $P_{\ell}$ and the ethanol concentration $f_{e}$ are in a moderate range - into the context of our model: For the no-bubble-regime at low laser powers $P_{\ell}$, the laser power is below the threshold for the nucleation of the plasmonic bubble at the given ethanol concentration [30]. Too high laser powers however lead to an unstable bubble behavior ("unstable regime"): For large ethanol concentration $f_{e}$, due to the massive vaporization of ethanol, new bubbles can already form before the thermal Marangoni forces have pulled back the former bubble to the substrate. This can lead to bubble collisions and thus instability. On the other hand, for ethanol concentrations $f_{e} \lesssim 66 \mathrm{wt} \%$, the strong solutal Marangoni force (reflected in a large $\gamma_{s}$ ) leads to a very high jumping height of the bubble, so that also here a new bubble can form before the return of the former bubble, see Eq. 4.3. Finally, in the pinned regime $f_{e} \gtrsim 82 \mathrm{wt} \%$, the ethanol concentration difference is so small that the solutal Marangoni force directing away from the substrate is too weak to depin the bubble from the substrate.

\subsection{Conclusions}

In conclusion, we have experimentally and theoretically studied the competition between thermal and solutal Marangoni forces acting on a nucleating plasmonic bubble in a binary liquid. This competition leads to a periodic bouncing of the bubble towards and away from the surface with a frequency of several $\mathrm{kHz}$. The phenomenon not only exemplifies the richness of phenomena which can occur in the physicochemical hydrodynamics of bubbles far from equilibrium and with phase transitions, but it is also very relevant way beyond the nucleation and early life of plasmonic bubbles in binary liquids: It can straightforwardly be generalized to the nucleation and early life of vapor bubbles in boiling phenomena in binary liquids or to electrolytically or catalytically generated bubbles in multicomponent liquids. For applications, the bouncing of these bubbles can presumably be a considerable advantage, leading to much better mixing in the liquid close to the substrate and therefore for higher efficiency in processes like electrolysis and catalysis. 


\subsection{Appendix}

\subsubsection{Methods and materials}

The preparation of sample with gold nanoparticle is shown shown in Figs. 2.1 (a) and (b) in Chapter 2. The experimental setup for plasmonic microbubbles imaging is shown in Fig. 3.1 in Chapter 3. The only difference is that the laser irradiation and vertical imaging are from the bottom side. Ethanol (SigmaAldrich; $\geq 99.8 \%$ ) and deionized (DI) water (Milli-Q Advantage A10 System, Germany) were mixed to prepare binary liquids. Before they were mixed, ethanol and water were kept in air for $8 \mathrm{hrs}$ to make them air saturated. Subsequently, they were mixed to obtained ethanol-water binary liquids with 15 different ethanol concentrations of $62 \%, 64 \%, 66 \%, 68 \%, 70 \%, 72 \%, 74 \%$, $75 \%, 76 \%, 78 \%, 80 \%, 82 \%, 84 \%, 86 \%, 88 \%$ (all weight). The mixed binary liquids were immediately transferred into a sealed cuvette for experiments.

\subsubsection{PIV measurement}

For the PIV measurements, fluorescent particles (Thermo Scientific, FluoroMax Orange, $1.1 \mu \mathrm{m}$ in diameter) were added into the ethanol-water binary liquid $\left(f_{e}=75 \mathrm{wt} \%\right.$ ), with particle concentration of $100 \mu \mathrm{g} / \mathrm{mL}$. These particles were activated by the same laser source as for plasmonic bubble nucleation and bouncing. The laser beam was tuned to a size larger than the bubble radius to obtain a large enough illuminated region, as shown in Fig. 4.4. The laser power applied in the measurement was $260 \mathrm{~mW}$. The SAZ high-speed camera equipped with the $10 \times$ objective was then used to record the images at a frame rate of $30 \mathrm{kfps}$. The images were processed by the PIVlab toolbox in MATLAB to obtain the flow field. Square interrogation windows of $32 \times 32$ pixels corresponding to grid cells of $53 \mu \mathrm{m} \times 53 \mu \mathrm{m}$ with an overlap of $50 \%$ were used to obtain the velocity vectors.

\subsubsection{Liquid vapor equilibrium and surface tension of binary liquids}

Figure 4.5 (a) shows the liquid vapor equilibrium curve of ethanol-water binary liquids as a function of ethanol concentration $f_{e}$, based on ref. [137]. The surface tension $\sigma$ as function of the ethanol concentration $f_{e}$ and temperature $T$ is shown in Fig. 4.5 (b), see reference [138]. The surface tension $\sigma$ gradually decreases with increasing temperature $T$. On the contrary, it rapidly decreases 


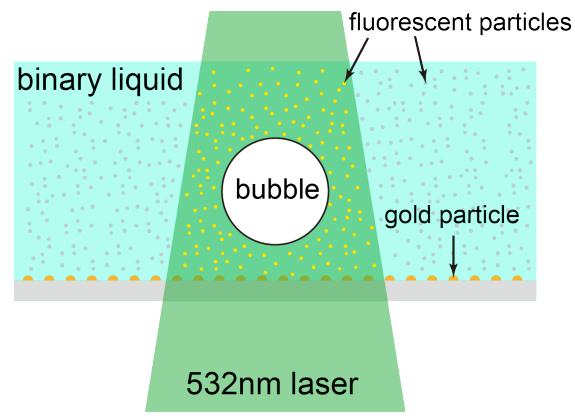

Figure 4.4: Schematic diagram of the PIV measurement of the bubble bouncing in the binary liquid.

with increasing $f_{e}$ for binary liquids at small ethanol fraction. This indicates that $\left|\partial \sigma / \partial f_{e}\right|$ is larger at small ethanol fractions than at high ethanol fractions.

(a)

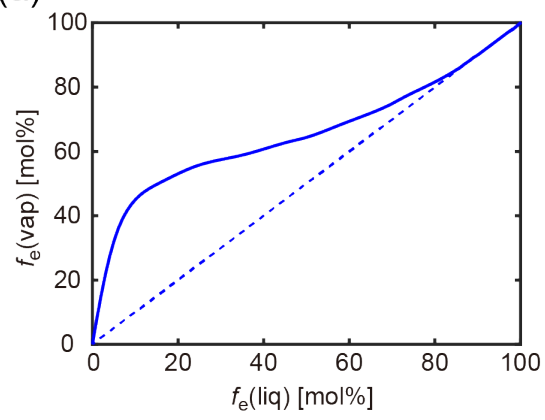

(b)

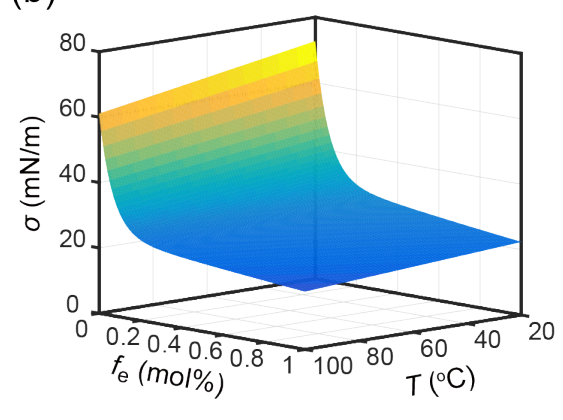

Figure 4.5: (a) Vapor-liquid equilibrium of ethanol water binary liquids. (b) Surface tension of the ethanol-water binary liquid as function of the ethanol concentration $f_{e}$ and the liquid temperature $T$.

\subsubsection{Bubble bouncing with inverted sample}

To confirm that the effect of gravity is negligible in the bubble bouncing, we performed one experiment in an inverted configuration with the gold nanoparticle decorated substrate upside down. This was achieved by attaching the sample on the top window of the cuvette. Because the substrate cannot be stably attached on the top window of the cuvette in ethanol-water binary liquids, here we used acetone-water binary liquids instead. The acetone concentration 
was $90 \mathrm{wt} \%$ and the laser power $P_{\ell}$ was $35 \mathrm{~mW}$. The bubble bouncing takes place in acetone-water binary liquids as well. The bouncing bubbles exhibit similar behaviors as in ethanol-water binary liquids.

The bubble radius $R$, the bubble center position $H_{C}$, and the bouncing frequency $f_{b}$ for the inverted configuration as well as two upright measurements are shown in Figs. 4.6 (a)-(c), respectively. One can see that $f_{b}$ has the similar distribution for both configurations, which implies that gravity does not play any role in the bubble bouncing process.

(a)

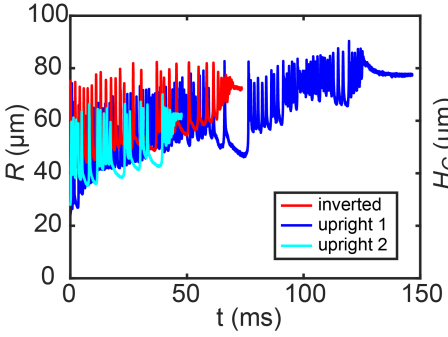

(b)

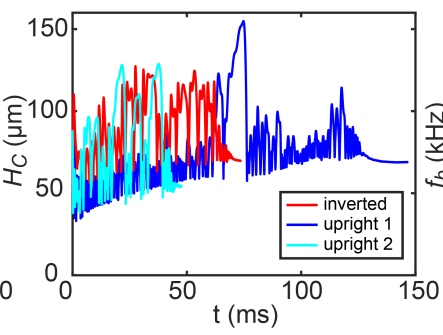

(c)

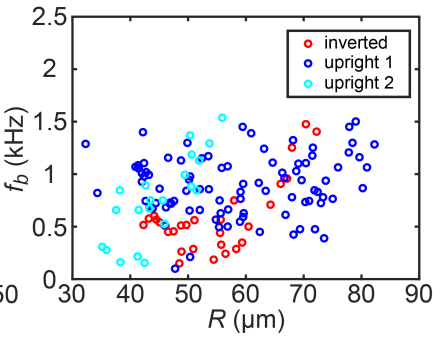

Figure 4.6: Comparison of bubble bouncing dynamics with the upright and inverted configurations. (a) and (b) The bubble radius $R$ and the center position $H_{C}$ versus time $t$ of the bouncing bubbles. (c) Bubble bouncing frequency $f_{b}$ versus bubble radius $R$. All bubbles exhibit similar dynamics, implying that the effect of the gravity in the bubble bouncing is negligible.

\subsubsection{Bullet model of the bouncing bubble}

\section{Bubble radius variations}

The variation of the bubble radius is quite large owing to the liquid vaporization and vapor condensation that occurs in every bouncing cycle. However, these two processes are very fast and occur when the bubble gets very close to the substrate. As shown in the Fig. 4.7, the strong bubble radius variations only involve a relatively small fraction (15\%) of a bouncing cycle. Neglecting the bubble size variation is therefore a reasonable assumption.

\section{Solution of the bullet model}

In the bullet model, the single bouncing process of the bubble is given by

$$
\frac{10}{3} \pi \rho R^{3} \ddot{z}(t)=-4 \pi \rho \nu R \dot{z}(t)-\gamma_{t h} R^{2},
$$




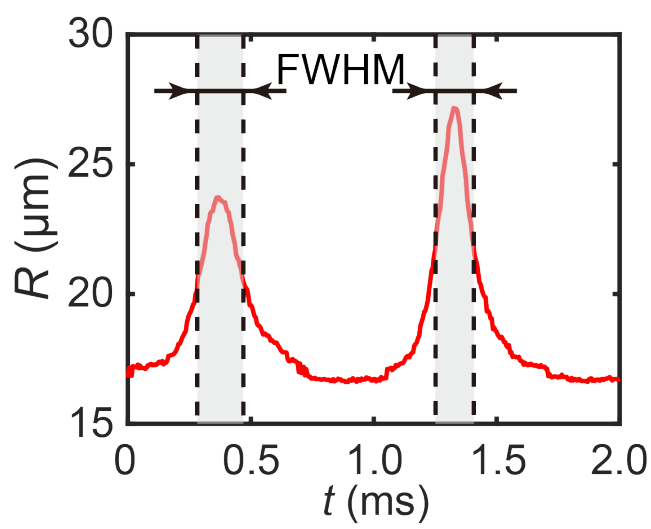

Figure 4.7: Bubble radius $R$ versus time $t$ for a bouncing bubble. The full width at half maximum (FWHM) of the bubble radius only involves a relatively small fraction of a bouncing cycle.

where $z(t)$ the center position of the bubble, $R$ the bubble radius, which is assumed to be constant, $\nu$ the kinematic viscosity of the liquid, $\rho$ its density, and $\gamma_{t h}$ the surface tension gradient caused by temperature gradient. This equation can be rewritten as

$$
\ddot{z}(t)=-\beta \dot{z}(t)-a_{t h},
$$

where $a_{t h}$ (the acceleration caused by thermal Marangoni force $F_{t h-M}$ ) is given by $a_{t h}=3 \gamma_{t h} /(10 \pi \rho R)$, and $\beta=6 \nu /\left(5 R^{2}\right)$ is the damping rate. The analytical solution of Eq. 4.7 with the initial conditions $z(0)=0, \dot{z}(0)=$ $\sqrt{3 \gamma_{s} \lambda_{s}^{3} /\left(5 \pi \rho R^{3}\right)}$ as given in the main text is given by

$$
z(t)=\frac{a_{t h}}{\beta^{2}}\left(1+\frac{\beta \dot{z}(0)}{a_{t h}}\right)\left(1-e^{-\beta t}\right)-\frac{a_{t h}}{\beta} t .
$$

A full bounce cycle is finished once $z\left(t_{d u r}\right)=0$ again. This gives the following implicit equation for the duration $t_{d u r}$ of one full cycle,

$$
\beta t_{d u r}=\left(1+\frac{\beta \dot{z}(0)}{a_{t h}}\right)\left(1-e^{-\beta t_{d u r}}\right) .
$$

Note that of course also $t_{d u r}=0$ is a solution of Eq. 4.9. By substituting $a_{t h}=3 \gamma_{t h} /(10 \pi \rho R)$ and $\beta=6 \nu /\left(5 R^{2}\right)$ into Eq. 4.9, we obtain the required 
travel time of the bubble for one full cycle,

$$
t_{d u r}=\frac{5 R^{2}}{6 \nu}\left(1+\sqrt{\frac{48 \pi}{5} \frac{\gamma_{s}}{\gamma_{t h}} \frac{\rho \nu^{2}}{\gamma_{t h} R^{2}} \frac{\lambda_{s}^{3}}{R^{3}}}\right)\left(1-e^{-\frac{6 \nu}{5 R^{2}} t_{d u r}}\right) .
$$

\section{Relationship between radius of minimum frequency and laser spot radius $R_{\ell}$}

Figure 4.8 depicts the schematics of the concentration boundary layer in the vicinity of the laser spot on the sample substrate. When the bubble radius $R$ is smaller than the laser spot radius $R_{\ell}$ [Fig. 4.8 (a)], $R$ itself is taken as length scale to estimate the thickness of the concentration boundary layer $\lambda_{s}$, given by $\lambda_{s}=k R / \sqrt{S c}[136]$, where $k$ is a prefactor by matching the theoretical bouncing frequencies of the bubble to the experimental ones. However, for $R \geq R_{\ell}$, the scale cannot reach $R$ due to the fact that the water rich area is limited by the laser spot size. Correspondingly, we take the laser spot radius $R_{\ell}$ as length scale to estimate $\lambda_{s}=k R_{\ell} / \sqrt{S c}$, as shown in Fig. 4.8 (b). Therefore, we arrive at $\lambda_{s}=k \min \left(R, R_{\ell}\right) / \sqrt{S c}$. With $k=23$, we obtain a qualitatively good agreement between theoretical and experimental bouncing frequencies, as shown in Fig. 4.3 (b) in the maintext.

(a) $R<R_{\ell}$

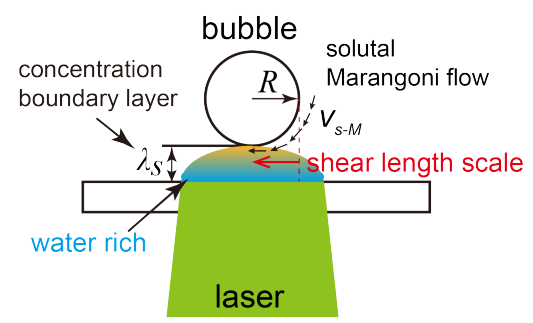

(b) $R \geq R_{\ell}$

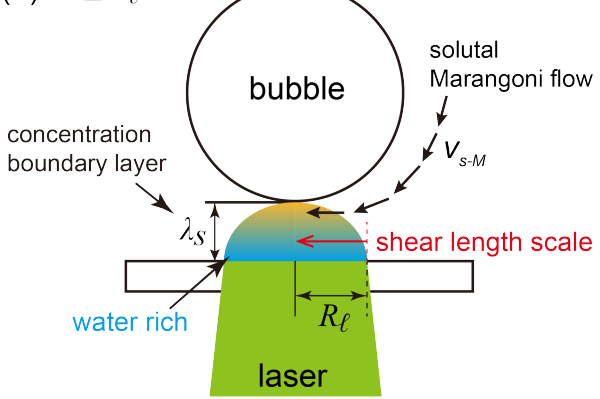

Figure 4.8: Schematics of the concentration boundary layers in the vicinity of the laser spot on the sample surface. (a) When $R<R_{\ell}$, the bubble radius $R$ is taken as the shear length scale for the estimation of concentration boundary layer thickness. (b) In contrast, when $R \geq R_{\ell}$, the laser spot radius $R_{\ell}$ is taken as the shear length scale.

To give further evidence that the diameter of the laser spot size plays an important role in the plasmonic bubble dynamics, we performed experiments 
with different laser spot sizes. The laser spot size was tuned to about 12 $\mu \mathrm{m}, 20 \mu \mathrm{m}$, and $30 \mu \mathrm{m}$ with a similar laser power density. The measured bubble bouncing frequency $f_{b}$ as function of $R$ for different $R_{\ell}$ is shown in Fig. 4.9. The results clearly show that the transition radius of the minimum bouncing frequency increases with increasing $R_{\ell}$, which complies with the physical picture proposed in the bullet model.

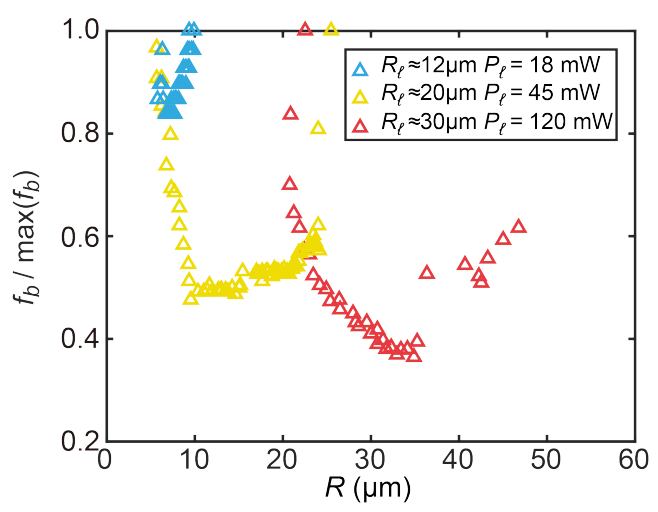

Figure 4.9: Normalized bouncing frequency $f_{b} / \max \left(f_{b}\right)$ as function of the bubble radius $R\left(f_{e}=80 \mathrm{wt} \%\right)$ for three different sets of control parameters $\left(R_{\ell}, P_{\ell}\right)$. In all cases a minimum in the bubble bouncing frequency can be identified.

\section{Estimation of surface tension gradients $\gamma_{s}$ and $\gamma_{t h}$}

Figure 4.10 (a) shows a schematic of a bouncing bubble with the concentration boundary layer and the thermal boundary layer. The thermal boundary layer thickness $\lambda_{t h}$ is much larger than the concentration boundary layer thickness $\lambda_{s}$. In that case the bubble is totally immersed in the thermal boundary layer but only partially immersed in the concentration boundary layer. Thus, the influence of the solutal Marangoni force can be regarded as a short impulse, as illustrated in Fig. 4.10 (b) (top). The impulse gives the bubble an initial velocity $\dot{z}(0)$. The thermal Marangoni force can then work as the restoring force and pull the bubble back. Figure 4.10 (b) (bottom) shows experimentally and theoretically obtained trajectories of bubble center position in a bouncing cycle at $f_{e}=75 \mathrm{wt} \%, R=20 \mu \mathrm{m}$, and $P_{\ell}=45 \mathrm{~mW}$. The theoretical one was obtained from Eq. 4.8. The two trajectories qualitatively agree with each other. 
In order to estimate the bouncing frequency $f_{b}$, the surface tension gradients $\gamma_{s}$ and $\gamma_{t h}$ caused by the ethanol concentration difference and the temperature difference, respectively, have to be estimated. We assume that both $\gamma_{s}$ and $\gamma_{t h}$ are constant at given radius $R$ and ethanol concentration $f_{e}$. The gradient $\gamma_{s}$ can be simplified by the ratio of the surface tension difference $\Delta \sigma$ between the bubble bottom and the bubble apex to the thickness $\lambda_{s}$ of the concentration boundary layer. Here, $\Delta \sigma$ depends on the difference between the ethanol concentration $f_{e-\text { bottom }}$ near the bubble bottom after selective vaporization and $f_{e}$ in the bulk. The value $f_{e-\text { bottom }}$ can be estimated by

$$
f_{e-b o t t o m}=\frac{f_{e}(l i q)-k_{\ell-v} f_{e}(v a p)}{1-k_{\ell-v}},
$$

where $f_{e}(l i q)$ is the original ethanol fraction near the bubble bottom, $f_{e}(v a p)$ the ethanol fraction in the vapor, and $k_{\ell-v}$ the mass ratio between the vaporized liquid and the total liquid near the bubble bottom during vaporization. The values $f_{e}(l i q)$ and $f_{e}(v a p)$ can be taken from Fig. 4.5(a). Note that $0<k_{\ell-v}<1$. By assuming an average $k_{\ell-v}$ of 0.5 , Eq. 4.11 reads $f_{e-\text { bottom }}=2 f_{e}(l i q)-f_{e}(v a p)$. Since $f_{e-\text { bottom }} \geq 0, f_{e-\text { bottom }}$ can be estimated by $f_{e-\text { bottom }}=\max \left[0,2 f_{e}(\right.$ liq $\left.)-f_{e}(v a p)\right]$. The obtained $f_{e-\text { bottom }}$ as function of $f_{e}$ is shown in Fig. 4.10 (c). It shows that when the original ethanol concentration $f_{e} \lesssim 0.5$, the liquid near the bubble bottom becomes pure water after the selective vaporization. When $f_{e} \gtrsim 0.5, f_{e-b o t t o m}$ increases with increasing $f_{e}$. With the obtained $f_{e-\text { bottom }}$, the surface tension gradient $\gamma_{s}$ can then be obtained from

$$
\gamma_{s} \approx \frac{\Delta \sigma}{\lambda_{s}}=\frac{\sigma\left(f_{e-b o t t o m}\right)-\sigma\left(f_{e}\right)}{\lambda_{s}},
$$

where $\sigma\left(f_{e-\text { bottom }}\right)$ and $\sigma\left(f_{e}\right)$ are the surface tensions of the liquid near bubble bottom and in bulk, respectively, which are taken from Fig. 4.5 (b) at room temperature $\left(20^{c}\right.$ irc $\left.\mathrm{C}\right)$. With $\lambda_{s} \approx 10 \mu \mathrm{m}, \gamma_{s}$ as function of $f_{e}$ is shown in Fig. 4.10 (d). It is found that for $f_{e} \lesssim 0.5, \gamma_{s}$ increases with increasing $f_{e}$, while for $f_{e} \gtrsim 0.5, \gamma_{s}$ decreases with increasing $f_{e}$. This is because the ethanol concentration difference $\left|f_{e}-f_{e-\text { bottom }}\right|$ increases with $f_{e}$ for $f_{e} \lesssim 0.5$, while decreases with $f_{e}$ for $f_{e} \gtrsim 0.5$. In our experiments, we focused on the range of $f_{e}>0.5$.

In order to obtain the surface tension gradient $\gamma_{t h}$ caused by the temperature gradient in the temperature boundary layer, we determined the liquid temperature $T_{s u b}$ near the substrate in a numerical approach, which is given in details in Eqs. 2.1 and 2.2 in Chapter 2. The temperature field after $1 \mathrm{~ms}$ 
(a)

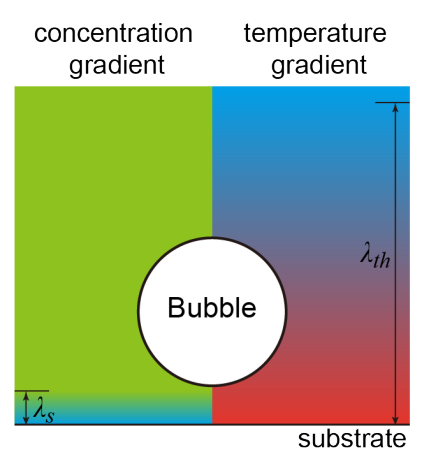

(c)

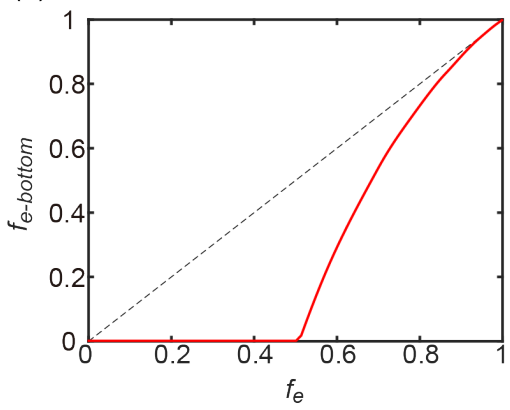

(b)

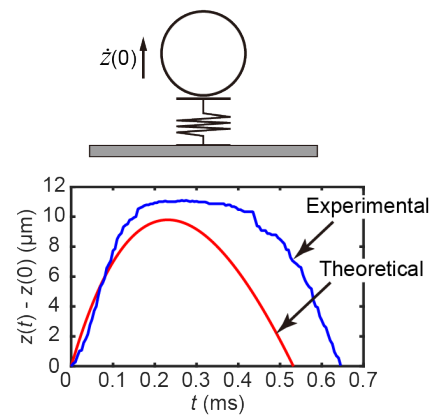

(d)

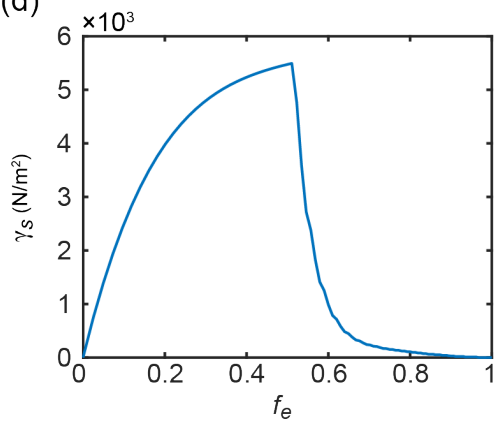

Figure 4.10: (a) Schematics of a bouncing bubble with the concentration boundary layer and the thermal boundary layer. (b) Schematics of the bullet model and the experimental and theoretical trajectories of the center position of a bouncing bubble in one cycle $\left(f_{e}=74 \mathrm{wt} \%, P_{\ell}=45 \mathrm{~mW}\right.$, and $R=20$ $\mu \mathrm{m})$. (c) Estimated ethanol concentration near the bubble bottom after selective vaporization of ethanol for different original ethanol concentrations $f_{e}$. (d) Estimated surface tension gradient $\gamma_{s}$ versus the original ethanol concentration $f_{e}$.

of laser irradiation with laser power $P_{\ell}=45 \mathrm{~mW}$ is shown in Fig. 4.11. From the simulation result we obtain a substrate temperature $T_{s u b} \approx 100{ }^{\circ} \mathrm{C}$, which is higher than the boiling point temperature of the ethanol-water binary liquid with $f_{e}=75 \mathrm{wt} \%$ (about $79^{\circ} \mathrm{C}$ ). Subsequently, the surface tension gradient $\gamma_{t h}$ in the thermal boundary layer can be obtained as

$$
\gamma_{t h} \approx \frac{\Delta \sigma}{\lambda_{t h}}=\frac{\sigma\left(T_{0}\right)-\sigma\left(T_{s u b}\right)}{\lambda_{t h}}
$$

where $T_{0}=20{ }^{\circ} \mathrm{C}$ is the room temperature. In the equation, $\sigma\left(T_{\text {sub }}\right)$ and 
$\sigma\left(T_{0}\right)$ can be obtained from Fig. 4.5 (b) at a typical ethanol concentration $f_{e}$ $=75 \mathrm{wt} \%$. Note again that the thermal boundary layer $\lambda_{t h}$ is much thicker than the concentration boundary layer $\lambda_{s}$ and the bubble radius $R$. Here, we roughly estimate $\lambda_{t h}$ as $100 \mu \mathrm{m}$ by using the relationship $\lambda_{t h}=\lambda_{s} \sqrt{L e}$, where we use $\lambda_{s}=10 \mu \mathrm{m}$ for the estimation, and $L e=110$ is the Lewis number. It leads to $\gamma_{t h} \approx 60 \mathrm{~N} / \mathrm{m}^{2}$. Here, we simply use $\lambda_{t h}=100 \mu \mathrm{m}$ for all cases. It is reasonable that the thermal boundary layer thickness $\lambda_{t h}$ is independent of $R$, since the thermal boundary layer is much thicker than the bubble and the relevant length scale is not set by the bubble radius $R$. We note that the qualitative trend of the bouncing frequency is not sensitive to the exact value of the chosen $\lambda_{t h}$.

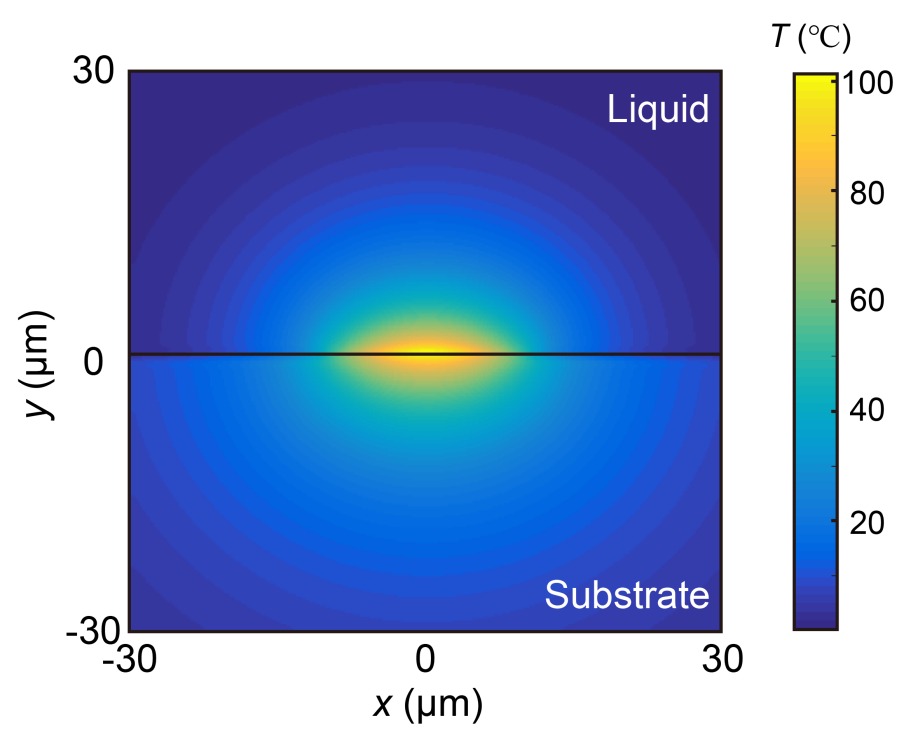

Figure 4.11: Temperature field of the ethanol-water binary liquid and the substrate in the vicinity of the laser spot. The temperature field was constructed by numerically solving the heat diffusion model (Eqs. 2.1 and 2.2 in Chapter 2) upon laser irradiation at $P_{\ell}=45 \mathrm{~mW}$ for $1 \mathrm{~ms}$ (see ref. [30] for more details). 


\section{Chapter 5}

\section{Plasmonic microbubble dynamics in binary liquids 1}

The growth of surface plasmonic microbubbles in binary water-ethanol solutions is experimentally studied. The microbubbles are generated by illuminating a gold nanoparticle array with a continuous wave laser. Plasmonic bubbles exhibit ethanol concentration dependent behaviors. For small ethanol concentration $f_{e} \lesssim 67.5 \%$, bubbles do not exist at the solid-liquid interface. For large $f_{e} \gtrsim 80 \%$, the bubbles behave as in pure ethanol. Only in an intermediate window $67.5 \% \lesssim f_{e} \lesssim 80 \%$, we find sessile plasmonic bubbles with a highly non-trivial temporal evolution, in which as function of time three phases can be discerned: (1) In the first phase the microbubbles grow, while wiggling, (2) as soon as the wiggling stops the microbubbles enter the second phase in which they suddenly shrink, followed by (3) a steady reentrant growth phase. Our experiments reveal that the sudden shrinkage of the microbubbles in the second regime is caused by a depinning event of the three-phase contact line. We systematically varied the ethanol concentration, the laser power, and the laser spot size to unravel water recondensation as the underlying mechanism of the sudden bubble shrinkage in phase (2).

\footnotetext{
${ }^{1}$ Published as: Xiaolai Li, Yuliang Wang, ${ }^{*}$ Binglin Zeng, Marvin Detert, Andrea Prosperetti, Harold J. W. Zandvliet, ${ }^{*}$ and Detlef Lohse, ${ }^{*}$, Plasmonic microbubble dynamics in binary liquids, J. Phys. Chem. Lett. 11, 8631-8637 (2020). Experiment (in part), Mechanism analysis (in part) and data processing (in part) preparation by Zeng.
} 


\subsection{Introduction}

Plasmonic microbubbles formed around metal nanoparticles immersed in liquid and irradiated by a resonant light source have numerous applications ranging from biomedical diagnosis and cancer therapy [27, 54, 82, 128], micromanipulation of micro/nano-objects [58, 139, 140], to locally enhanced chemical reactions [21, 77]. A proper understanding of the plasmonic microbubble dynamics is crucial for the optimal usage of these bubbles in the aforementioned applications.

In a previous study, we have shown that plasmonic microbubbles in pure (mono-component) liquids undergo four different phases: the initial giant vapor bubble (phase I), the oscillating bubble (phase II), the water vaporization dominated growth (phase III), and the air diffusion dominated growth (phase IV) [30]. The bubble dynamics in these different phases depends on several factors, such as particle arrangement [58], laser power, gas concentration [30,32,62], and types of liquid [31, 141]. The nucleation of a giant bubble is initiated by locally heating the liquid around the plasmonic particle up to the spinodal temperature [30]. The heating process is limited by thermal diffusion and can theoretically be well described [71, 142, 143]. Moreover, the dissolved gas plays a major role in bubble nucleation: an increase of the relative gas concentration leads to a lower bubble nucleation temperature [30, 32]. The radius of the bubble in the air diffusion dominated phase IV is $R(t) \propto t^{1 / 3}$ for air equilibrated or air-oversaturated water [32, 62]. Furthermore, several studies on the shrinkage of bubbles have revealed that the shrinkage depends on the illumination history of the bubble as well as the gas concentration level of the liquid [33, 77].

Most of these studies were conducted in water or other pure liquids [141], such as n-alkanes [31]. However, it is known that many plasmonic assisted processes also take place in binary liquids [21]. However, the dynamics of plasmonic bubbles in binary liquids has not yet been investigated in detail. Only in a recent study, we started to investigate the formation of the initial giant plasmonic bubbles in binary liquids [127]. It was shown that the dynamics of bubble nucleation depends on the exact composition of the binary liquid, which could be rationalized based on the constituents of the binary liquid exhibiting different physicochemical properties, such as boiling point and latent heat. In the aforementioned study the focus was only on phase I of the plasmonic bubble formation process, namely, bubble nucleation. A thorough and complete understanding of the full long-term growth dynamics 
of plasmonic bubbles in binary liquids is still lacking.

In this letter, we study the growth dynamics of plasmonic bubbles in ethanol/water mixtures over a large range of parameters and over the full bubble lifetime cycle. The bubble dynamics in various ethanol/water mixtures was monitored by two high speed cameras: one provides a side view and the other the bottom view, both at a frame rate of $1000 \mathrm{fps}$ (see Experimental Methods section in the Appendix for details).

\subsection{Methods and Materials}

The preparation of sample with gold nanoparticle is shown shown in Figs. 2.1 (a) and (b) in Chapter 2. The experimental setup for plasmonic microbubbles imaging is shown in Fig. 3.1 in Chapter 3. The difference is that the laser irradiation and vertical imaging are from the bottom side. During experiments, the nanoparticle decorated sample surface was immersed into binary liquids of ethanol (Sigma-Aldrich; $\geq 99.8 \%$ ) and deionized (DI) water (Milli-Q Advantage A10 System, Germany). The ethanol and water were kept open in air for 8 hours to make them air saturated. After that, they were mixed to prepare binary liquids with ethanol ratios of $20 \%, 40 \%, 60 \%, 65 \%, 67.5 \%$ $80 \%$ with a step of $2.5 \%, 85 \%$, and $90 \%$. The obtained binary liquids were then immediately transferred into a sealed cuvette for experiments. Since the air solubility in ethanol is much higher than that in water, the prepared binary liquids must be air saturated as well.

\subsection{Results and discussion}

We first report the plasmonic bubble behavior as a function of the ethanol concentration $f_{e}$, see Fig. 5.1. First, in binary liquids with ethanol concentrations $0<f_{e} \lesssim 67.5 \%$ (weight), the bubbles are unstable and not pinned at the substrate. As an example, in Fig. 5.1(a) (left column) we show the bubble dynamics for an ethanol concentration of $40 \%$. In this regime, after nucleation, the bubble detaches from the substrate. Subsequently, a new bubble is generated and then also detaches.

Next, at ethanol concentrations $67.5 \% \lesssim f_{e} \lesssim 80 \%$, we find that the plasmonic bubble does not detach from the substrate. As an example for this regime, the dynamics for $f_{e}=70 \%$ is shown in Fig. 5.1(a) (right column). Instead, after an initial growth phase, the plasmonic bubble starts to wiggle in this regime, with gradually increasing radius $R$ (Fig. 5.1(b) (I)), considerably 

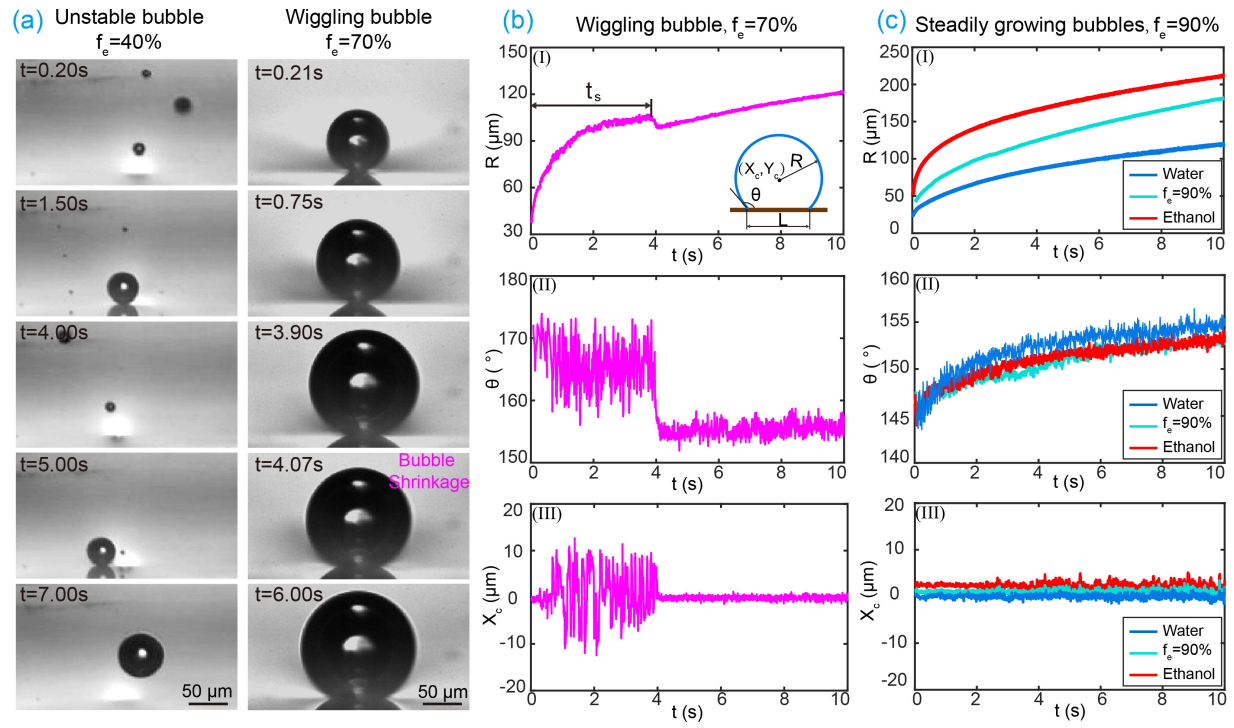

Figure 5.1: (a) Dynamics of plasmonic bubble growth in ethanol-water mixtures with ethanol concentrations of $f_{e}=40 \%$ (left) and $f_{e}=70 \%$ (right). In the binary liquid with $f_{e}=40 \%$, the bubble detaches from the substrate after nucleation, whereas in the binary liquid with $f_{e}=70 \%$, the bubble remains pinned on the substrate and gradually grows. A sudden shrinkage is observed at time $t_{s}=4.07 \mathrm{~s}$. (b) The radius $R(t)(\mathrm{I})$, contact angle $\theta(t)$ (II), and horizontal center position $X_{c}(t)$ (III) of the bubble in the binary liquid with $f_{e}=70 \%$. The inset in (I) shows a sketch, which defines the radius $R$, the contact angle $\theta$, the horizontal center position $X_{c}$, and the footprint diameter $L$ of the bubbles. (c) $R(t), \theta(t)$ and $X_{c}(t)$ for a bubble in a binary mixture with $f_{e}=90 \%$, compared to the cases for pure water and pure ethanol. The laser power is $P_{\ell}=100 \mathrm{~mW}$ in all cases of Figs. 1a, b, c.

varying contact angle $\theta$ (Fig. 5.1(b) (II)) and horizontal center position $X_{c}$ (Fig. 5.1(b) (III)). During the first few seconds after nucleation, the radius, contact angle and horizontal center position exhibit an erratic behavior. At $t=4.07 \mathrm{~s}$ after bubble nucleation at $t=0 \mathrm{~s}$, the bubble abruptly stops wiggling and simultaneously starts to shrink for a short time. Afterwards, the bubble continues to steadily grow without exhibiting any wiggling. The abrupt stop of wiggling and the simultaneous starting sudden shrinkage always occur in 
the regime $67.5 \% \lesssim f_{e} \lesssim 80 \%$. We define the moment when this occurs as time of shrinkage $t_{s}$.

Finally, when the ethanol concentration $f_{e}$ of the binary liquids is higher than $80 \%$, we observed stable bubble growth, like in pure water and pure ethanol. Fig. 5.1(c) shows the bubble dynamics in pure water, in pure ethanol and in a binary liquid with $f_{e}=90 \%$. The laser power $P_{\ell}$ was maintained at $100 \mathrm{~mW}$ for these three measurements. In these three cases, the bubbles grow monotonously, with gradually increasing $R$ (Fig. 5.1 (c) (I)). No visible intermediate shrinkage and wiggling (Figs. 5.1 (c) (II) and (III)) were observed. Moreover, the radii of the bubbles increase with increasing ethanol concentration. As known from ref. [62], for air saturated water, the observed long-term growth is dominated by gas-diffusion. However, for the binary liquids in the regime of $f_{e} \gtrsim 80 \%$, bubble growth is dominated by the vaporization. Since the gas solubility in ethanol increases with temperature, the gas expelled from the liquid evaporation can be dissolved into liquids again, which has been shown in a recent study in n-alkane [31].

The above results demonstrate that the ethanol concentration in waterethanol mixtures plays a pivotal role in the bubble growth. Our interpretation of the observed phenomenon is as follows: in binary liquids with small ethanol ratios $f_{e} \lesssim 67.5 \%$, the detachment of bubbles from the substrate is caused by solutal Marangoni flow [124]. Because ethanol has a lower boiling temperature than water, at the hot substrate it evaporates much faster than water, leading to an ethanol depleted region near the three-phase contact line (triple-line) of the bubbles. The liquid at the top of the bubble is ethanol rich and thus has a smaller surface tension than the ethanol depleted region at the bottom of the bubble, resulting in a downward Marangoni flow. This solutal Maragoni flow pushes the bubble upwards, similarly to what has been observed for a jumping droplet in an ethanol-water concentration gradient [144]. As a result, the bubble detaches when the Marangoni flow is strong enough to overcome the pinning of the bubble at the surface.

The proposed mechanism for bubble detachment can be quantitatively validated. Here we consider an unstable bubble in binary liquids with an ethanol concentration $f_{e} \approx 40 \%-50 \%$ as an example. The surface tension difference $\triangle \sigma$ between the bubble top $\left(f_{e} \approx 40 \%-50 \%\right)$ and bubble bottom (providing $f_{e}=0 \%-20 \%$ ) is $\triangle \sigma \approx 20-40 \mathrm{mN} / \mathrm{m}$. For a bubble with a radius $R \approx 50 \mu \mathrm{m}$, the magnitude of the upward Marangoni force is $F_{M} \approx \triangle \sigma R=1 \times 10^{-6}-2 \times 10^{-6} \mathrm{~N}$. The Marangoni force competes with the vertical capillary force $F_{p}$. Since the measured contact angle of water 
$\alpha=29.1^{\circ}$ (Fig. 5.9(a) in the Appendix) on our substrate is in the same ballpark as that on a smooth glass surface $\left(\alpha=24^{\circ}\right)$ [145], the influence of the gold nanoparticles on the pinning/capillary force of the substrate is very limited. As a result, it is reasonable to estimate the vertical capillary force by $F_{p} \approx \sigma \pi L \sin \alpha \approx 1.4 \times 10^{-6} \mathrm{~N}$, assuming that the substrate is homogeneous [146, 147]. In this expression, the surface tension $\sigma=50 \mathrm{mN} / \mathrm{m}$, the medium value of measured contact angle (gas side) $\theta=160^{\circ}$, and the perimeter of the three-phase contact line $\pi L=85 \mu \mathrm{m}$.

Notably, the surface tension of the ethanol-water mixtures changes rapidly for small ethanol fractions but remains almost constant for large ethanol fractions [94, 148] (see Fig. 5.6 in the Appendix). This feature explains the observed peculiar behavior: For pure water, the bubble remains pinned because there is no downward solutal Marangoni flow, but an upward thermal Marangoni flow. For low ethanol concentration $f_{e} \lesssim 67.5 \%$, due to the strong dependence of the surface tension on $f_{e}$ in this regime, a large gradient in surface tension can be created and the resulting Marangoni flow is strong enough to depin the bubbles. With increasing ethanol fraction, the surface tension gradient across the bubble and thereby the Marangoni flow become weaker until finally, at $f_{e} \approx 67.5 \%$, it is unable to overcome the pinning. Therefore, for that ethanol concentration a transition from detaching bubbles to pinned bubbles with stable growth takes place.

However, this mechanism still does not explain the observed wiggling and sudden shrinkage of the stable bubbles in the regime $67.5 \% \lesssim f_{e} \lesssim 80 \%$. In order to find out the physical mechanism for the sudden bubble shrinkage, from the bottom view we tracked the changes of the triple-line of a bubble during the growth. Figure 5.2(a) shows the sequential side view images (first row) and bottom view images (second row) of a bubble in a binary liquid with $f_{e}=70 \%$. The bubble shrinks in Fig. 5.2(a) (IV). In the bottom view images, a dry region (the light grey center area) can be observed within the tripleline. The dry area becomes circular and increases after the sudden shrinkage $(\mathrm{IV} \rightarrow \mathrm{V})$. Figure 5.2(b) shows the profile of the triple-line as extracted from the bottom view as well as the spot profile of the laser.

It is found that the triple-line is first pinned at the edge of the laser spot (the first column of Fig. 5.2(a) (I)), and then partially depins (Figs. 5.2(a) (II) and (III)). This explains the wiggling behavior of the bubble shown in Fig. 5.1(b). When the contact line depins completely from the laser spot region (Fig. 5.2(b) (IV)), the bubble shrinks. Afterwards, the triple-line remains circular and gradually expands, as shown in Fig. 5.2(b) (V). 

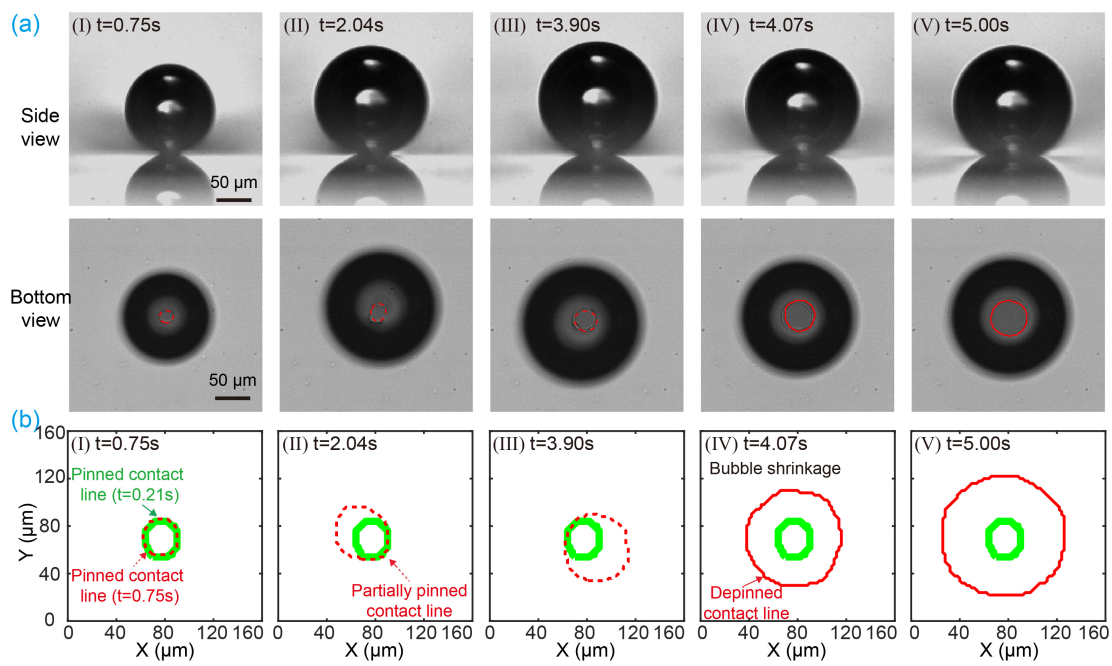

(c)
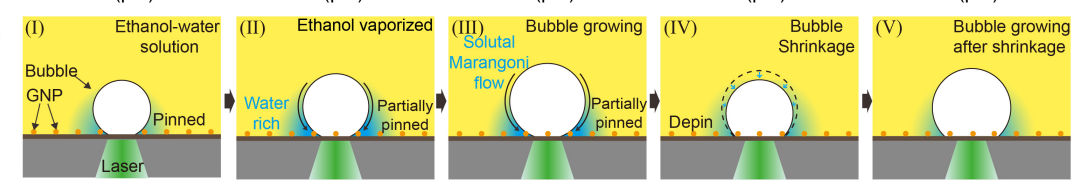

Figure 5.2: Dynamics of the bubble shrinkage. (a) Sequential side and bottom view images of a stable bubble in binary liquids with $f_{e}=70 \%$. The bubble shrinkage occurs in Fig. 5.2(a) (IV). (b) The three-phase contact line of bubbles extracted from the bottom view images of Fig. 5.2(a). The threephase contact line is initially pinned, and later partially pinned, at the edge of the laser spot (green line). At the moment $t_{s}$ of sudden shrinkage at IV, the bubble completely depins. After that, the contact area remains circular with increasing radius. (c) Schematics of bubble growth and shrinkage in a binary liquid.

The whole process of bubble growth and shrinkage is schematically shown in Fig. 5.2 (c). In the early stage of bubble growth, the evaporation of the more volatile ethanol in the binary liquids governs the bubble growth. As a result, the water concentration around the three-phase contact line, of which the size is defined by the laser spot, is relatively higher than the bulk binary liquid. At the triple line, the rapidly increasing bubble volume tends to expand the bubble and thus moves the triple line outwards. At the same time, the higher water concentration around the triple-line (due to the ethanol evaporation) leads to a locally increased surface tension, resulting in an increased receding 
contact angle [149, 150]. This helps to make the triple line stay pinned. The two effects compete. When the increased surface tension dominates, the tripleline remains pinned. Because of the continuous evaporation of the surrounding liquids, the bubble keeps growing. Finally, the contact angle (liquid side) reaches its receding value and the contact line begins to depin. During this process, the contact line is first partially depinned. Finally, once the water around the laser spot region is completely evaporated, complete depinning takes place, exhibiting a rapid decrease of the contact angle (gas side).

After depinning of the three-phase contact line, the region of the laser spot on the sample substrate is isolated by the bubble from the liquid. Owing to this effect, the light-vapor conversion efficiency significantly reduces and part of the vapor inside the bubble condenses, resulting in a sudden shrinkage of the bubble (Fig. 5.1(b) (I)). A numerical calculation (Fig. 5.5 in the Appendix) shows that the sample substrate remains non-isothermal in the vicinity of the triple-line when the rapid shrinkage takes place. This means that once the bubble depins, the temperature will drop at the triple-line, resulting in vapor condensation and hence rapid bubble shrinkage. The phenomenon resembles similar jumps in the contact angle occurring for evaporating [151-155] or dissolving binary droplets [107]. After the shrinkage, the bubble enters a steady growth phase again, but now with a lower optothermal conversion efficiency, during which there is no visible shrinkage happening again.

Note that the bubble shrinkage due to the pinning effect only appears in the binary liquids with intermediate ethanol ratios $67.5 \% \lesssim f_{e} \lesssim 80 \%$. In general, considering that the pinning force depends on the wettability and the roughness of the surface, the three-phase contact line motion must be expected to move in the 'stick-slip' mode [156 160], made up of one or 'more' stick phases, in which the contact line is pinned. However, in contrast to this expectation, the bubble growth and the contact angle in pure ethanol and pure water seem to exhibit monotonous growth in our experiments, as seen in Fig. 5.1(c). We interpret this finding that for the bubbles in the pure liquids, the pinning effect is only minor and not visible, and the 'stick' phase duration is so short that with our temporal resolution of $1000 \mathrm{fps}$, it is invisible in the measured dynamical process.

We now further verify the mechanism of bubble shrinkage by varying the laser power $P_{\ell}$ and the laser spot size. Figure 5.3(a) shows the bubble radius versus time at different laser powers for $5 \times$ and $10 \times$ objectives for laser focusing implying two different laser spot sizes (See Figs. 5.7 (a) and (b) for reproducible results of $R(t)$ in the Appendix). In Fig. 5.3(b) the delay time 
$t_{s}$ is plotted versus the laser power. For a fixed laser power, the delay time of the $5 \times$ objective is substantially longer than that of the $10 \times$ objective due to the weaker focusing of the laser beam, resulting in a larger laser spot and lower power density.
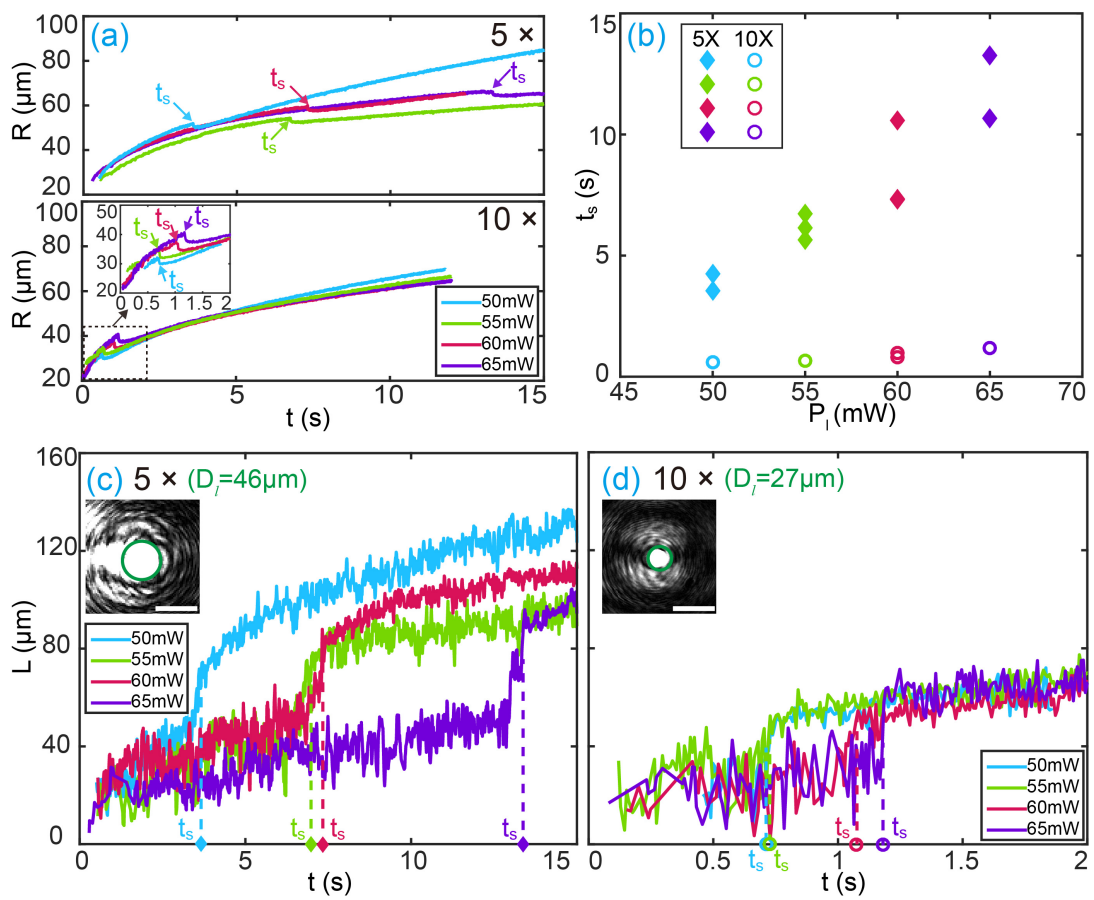

Figure 5.3: Dependence of the bubble shrinkage dynamics on laser power $P_{\ell}$ and laser spot size $D_{\ell}$ at $f_{e}=70 \%$. (a) Bubble dynamics for different laser powers $P_{\ell}$ at $5 \times$ and $10 \times$ objectives for laser focusing. (b) Delay time, $t_{s}$, as function of laser power. (c, d) Footprint diameter of the bubble versus time for $5 \times(\mathrm{c})$ and $10 \times(\mathrm{d})$ objectives. The insets show the laser spots. Scale bars: $50 \mu \mathrm{m}$.

The differences in the observed start moment of shrinkage $t_{s}$ can be understood by capturing the dynamics of the three-phase contact lines for the $5 \times$ and $10 \times$ objectives, see Figs. 5.3(c) and (d) (see also Fig. 5.8 for contact line $L$ as a function of radius $R$ in the Appendix). After a slight monotonous increase of the footprint diameter of the bubble, it exhibits a sudden jump and eventually gradually increases again. The sudden jump in the footprint diameter coincides with the depinning and shrinkage of the bubble. For the $5 \times$ 
and $10 \times$ objectives the jump in the footprint diameter occurs at about $50 \mu \mathrm{m}$ and $30 \mu \mathrm{m}$, respectively. These values agree well with the laser spot diameters $D_{\ell}=46 \mu \mathrm{m}$ and $27 \mu \mathrm{m}$ for the $5 \times$ and $10 \times$ objectives, respectively, as shown in the insets of Figs. 5.3(c) and (d). This also confirms that the triple-lines are initially pinned by the laser spot and shrinkage occurs because of complete depinning. The laser spot size increases with increasing laser power. As a result, the heated region and therefore the pinning radius increases. Thus, the delay period is longer for higher laser powers as more liquid needs to be evaporated to exceed the pinned region.

Finally, we have investigated the dependence of the bubble shrinkage dynamics on the ethanol concentration. As shown in Fig. 5.4(a), the bubble shrinkage is observed for ethanol concentrations varying from $70 \%$ to $80 \%$ (See Figs. 5.7 (c) and (d) of the Appendix for the reproducible results with different $f_{e}$ at laser powers of $60 \mathrm{~mW}$ and $100 \mathrm{~mW}$, demonstrating that this behavior is robust and reproducible.). Figure 5.4(b) shows the footprint diameter versus time. As we have kept the laser power and laser spot size constant, the footprint diameter at which the sudden shrinkage occurs is almost the same for all ethanol concentrations. Interestingly, the time $t_{s}$ of shrinkage steadily decreases with increasing ethanol concentrations (Fig. 5.4(c)).

The decrease of $t_{s}$ with increasing ethanol concentration is attributed to the fact that the dew point of the ethanol-water mixture decreases with increasing ethanol concentration, see Fig. 5.4.(d) [161, 162]. From the phase diagram it is immediately clear that at a higher ethanol concentration the ethanol can be evaporated at lower temperatures. Since the laser power and laser spot size are kept constant in this series of experiments, we can safely assume that the heating efficiency remains constant. Therefore, less energy and thus less time is required to evaporate ethanol for a binary liquid with a higher ethanol concentration as compared to a binary liquid with a lower ethanol concentration. The phase diagram also shows that the water/ethanol mixture becomes azeotropic at ethanol concentration higher than 90\%, implying that the binary liquid starts to behave as a pure liquid. This perfectly agrees with our experimental findings shown in Fig. 5.1(c), as beyond $f_{e} \approx 90 \%$ the plasmonic bubble behaves as in pure ethanol.

\subsection{Conclusions}

In summary, we have shown that the growth dynamics of plasmonic microbubbles in water-ethanol binary liquids strongly depends on the ethanol concen- 

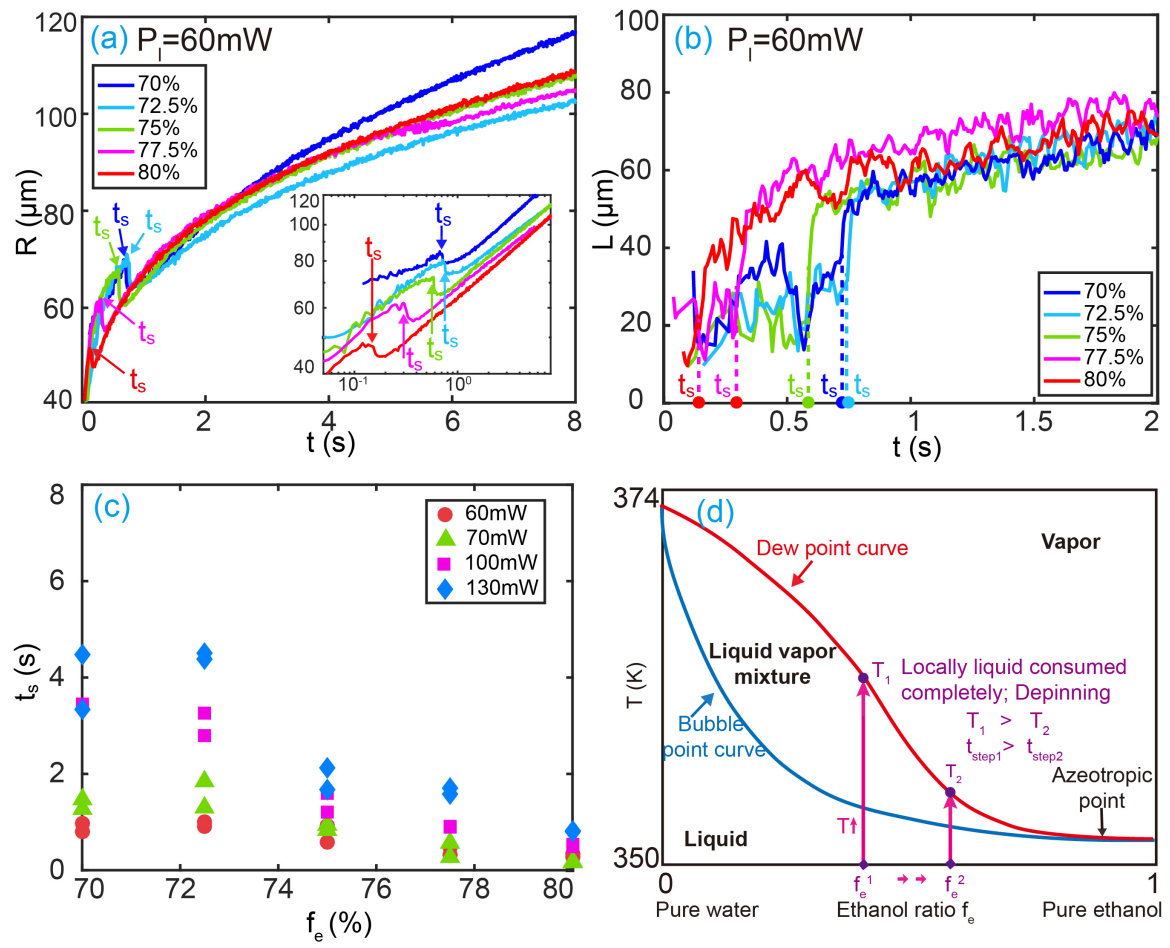

Figure 5.4: Bubble shrinkage dynamics for various ethanol concentrations in the stable bubble regime $67.5 \% \lesssim f_{e} \lesssim 80 \%$. (a) Radius $R(t)$ and (b) footprint width $L(t)$ at a laser power of $60 \mathrm{~mW}$. (c) The delay time $t_{s}$ of bubble shrinkage versus ethanol ratio $f_{e}$ for laser powers of $60 \mathrm{~mW}, 70 \mathrm{~mW}, 100 \mathrm{~mW}$, and 130 $\mathrm{mW}$. (d) Schematic diagram of the bubble point and dew point of ethanolwater mixtures, at ambient pressure.

tration. At ethanol concentrations below $67.5 \%$ bubbles nucleate and subsequently detach from the substrate due to the downward Marangoni flow. For ethanol concentrations in the range from $67.5 \%$ to $80 \%$, the bubbles first wiggle. A sudden bubble shrinkage takes place after a delay time $t_{s}$, originating from the complete depinning of the wiggling bubble from the laser spot area on the sample surfaces. Prior to that event the bubble was pinned or partially pinned on the laser spot. When the liquid around the pinned region is completely evaporated, the instantaneous expansion of the three-phase contact line as well as the sudden shrinkage takes place. Finally, when $f_{e} \gtrsim 80 \%$, the binary solutions become azeotropic, leading to a similar behavior as in pure 
liquids, and bubbles steadily grow. The knowledge of the sensitive dependence of the plasmonic bubble dynamics on the liquid compositions obtained in this paper may help in exploiting the relevant applications of these bubbles in multiple-component liquids. E.g., when a good local mixing of the binary liquids is preferable, operating in the first regime $f_{e} \lesssim 67.5 \%$, in which the bubble is wiggling, may be preferable. Having understood features is also a prerequisite to exploit them in optimizing the energy conversion efficiency of plasmonic bubble generation.

\subsection{Appendix}

\subsubsection{Temperature field of a silica substrate with a gold nanopar- ticle array}

A numerical calculation was performed to study the effect of the heat conductivity of the substrate (fused silica: $1.4-1.5 \mathrm{~W} / \mathrm{mK}$ for $293 \mathrm{~K}-373 \mathrm{~K}$ ) on the temperature field in the vicinity of the three-phase contact line (triple-line) of the bubbles. Details of the method can be found in our recent publication (PNAS 2018(115), 7676). Using this method, the spatial-temporal evolution of the substrate temperature can be obtained. Since the surrounding fluid (either vapor or water) has an influence on the temperature field of the substrate, we have considered two critical cases. In case 1 the surrounding fluid is vapor, whereas in case 2 the surrounding fluid is water. The temperature fields after 10s are shown in Figs. 5.5(a) and (b), respectively. In both cases there is a

(a)

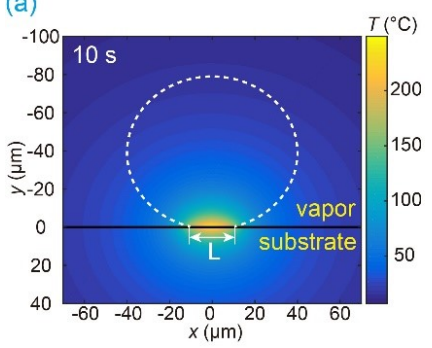

(b)

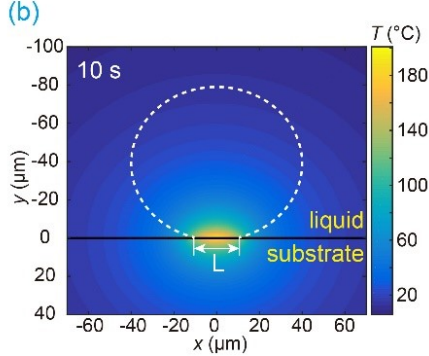

(c)

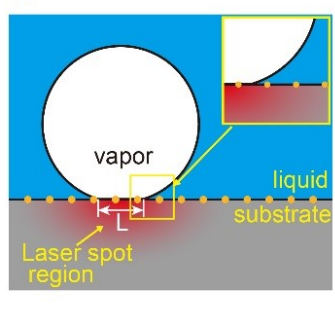

Figure 5.5: Numerical calculated temperature field of the substrate exposed to vapor (a) and immersed in liquid (b) under continuous laser irradiation for 10s. (c) Schematics of the temperature field. 
substantial temperature gradient at the location of the laser spot. This means that the substrate is not isothermal during the first $10 \mathrm{~s}$. Consequently, once the bubble depins, the temperature will drop at its triple-line, resulting in vapor condensation and hence shrinkage of the bubble.

In the actual experiments, obviously the substrate was neither completely immersed in water, nor fully exposed to vapor. As shown in Fig. 5.5(c), the substrate within the triple-line region is in contact with the vapor, whereas the rest of the substrate is in contact with water. Due to the large difference in the heat conductivity of vapor and water, the temperature gradient of the substrate around the triple-line is even more pronounced than shown in Figs. 5.5(a) and 5.5(b).

\subsubsection{Surface tension of ethanol and water mixtures}

Figure 5.6 shows the surface tensions of the ethanol-water binary liquid with ethanol weight ratio $f_{e}$ from 0 to 1 at $20^{\circ} \mathrm{C}$, at $50{ }^{\circ} \mathrm{C}$, and at boiling temperature (which depends on $f_{e}$ ). For each of the three curves, the surface tension $\sigma$ of the binary liquid decreases with the ethanol ratio $f_{e}$. It is also seen that $\sigma$ changes rapidly for small ethanol fractions and more slowly for larger ethanol fractions. The surface tensions for $f_{e}=67.5 \%$ and $f_{e}=80 \%$ are shown in Table 5.1.

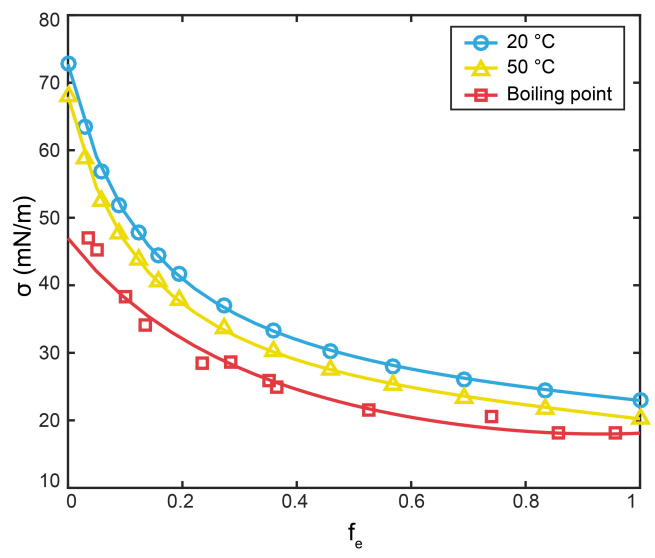

Figure 5.6: Surface tension of ethanol-water binary mixtures as a function of fe at $20^{\circ} \mathrm{C}$, at $50{ }^{\circ} \mathrm{C}$, and at the respective boiling temperature of the mixture (which depends on $f_{e}$ ). 
Table 5.1: Surface tension of binary liquids with ethanol ratio $f_{e}=67.5 \%$ and $f_{e}=80 \%$.

\begin{tabular}{lcc}
\hline Temperature & $\sigma\left(f_{e}=67.5 \%\right), \mathrm{mN} / \mathrm{m}$ & $\sigma\left(f_{e}=80 \%\right), \mathrm{mN} / \mathrm{m}$ \\
\hline $20{ }^{\circ} \mathrm{C}$ & 26.46 & 24.94 \\
$50{ }^{\circ} \mathrm{C}$ & 23.8 & 22.31 \\
Boiling point & 19.46 & 18.39 \\
\hline
\end{tabular}

\subsubsection{Robustness and reproducibility of the bubble shrinkage measurements}
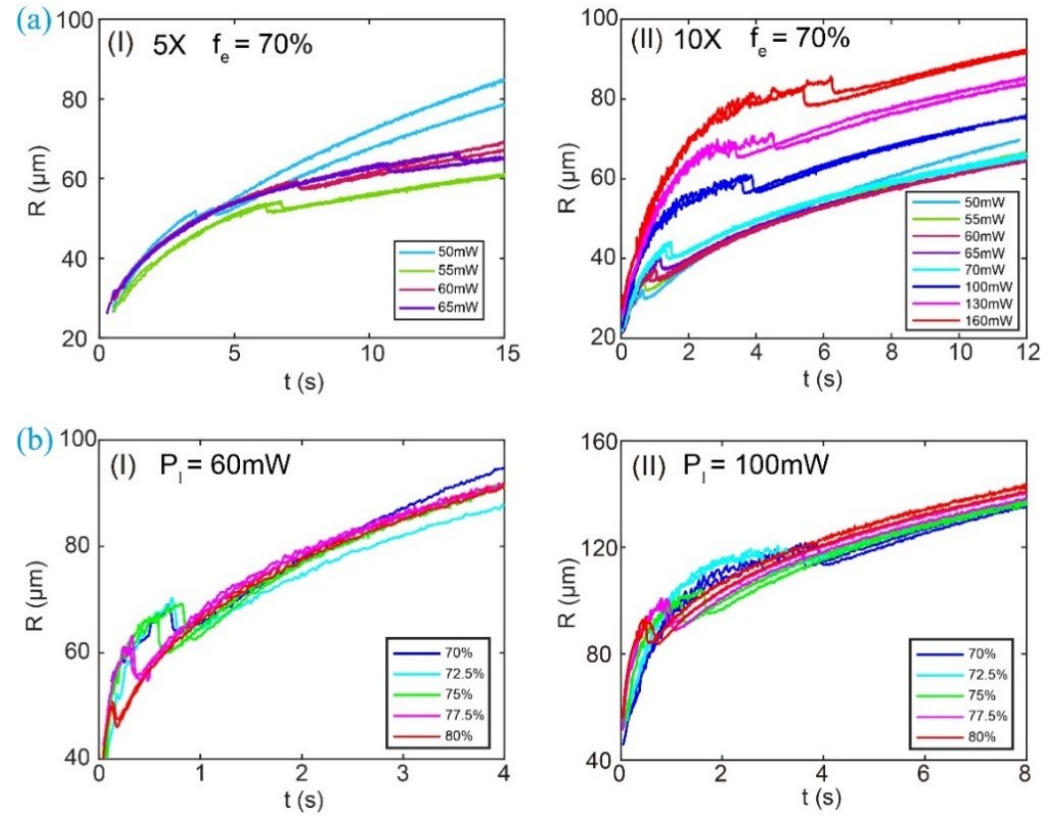

Figure 5.7: Repeated experimental results of the bubble shrinkage dynamics. (a) The bubble shrinkage dynamics for different laser powers using the $5 \times$ objective (I) and the $10 \times$ objective (II) at an ethanol concentration of $f_{e}=$ $70 \%$. (b) Bubble shrinkage dynamics for various ethanol concentrations in the regime of $67.5 \% \lesssim f_{e} \lesssim 80 \%$ at laser powers of $60 \mathrm{~mW}$ and $100 \mathrm{~mW}$, respectively.

Figures 5.7 (a) and (b) show repeated experimental results with the $5 \times$ (Fig. 5.7 (a) (I)) and $10 \times$ (Fig. 5.7 (a) (II)) objectives at an ethanol concentration 
$f_{e}=70 \%$ and at different ethanol concentrations for fixed laser powers of 60 $\mathrm{mW}$ (Fig. 5.7(b) (I)) and $100 \mathrm{~mW}$ (Fig. 5.7(b) (II)), respectively. Clearly, the dynamics of the bubble shrinkage at different laser powers and ethanol concentrations is robust and highly reproducible (see also Figs. 5.3(b) and 5.4 (c) in the paper).

\subsubsection{Dynamics of three-phase contact line and bubble radius}

The dynamics of the three-phase contact line (triple-line) during bubble growth is shown in Fig. 5.8. For both the $5 \times$ and $10 \times$ magnification objective of laser focusing, the triple-line first slightly increases with the increasing bubble radius. The triple-lines jump at about $50 \mu \mathrm{m}$ and $30 \mu \mathrm{m}$ for the $5 \times$ and $10 \times$ objectives, respectively, as shown in Fig. 5.8. Thus, right after the jump, the radii of bubbles are slightly smaller than before. After that, the bubbles enter the steadily growing phase and the triple-line gradually moves outwards along with bubble growth.
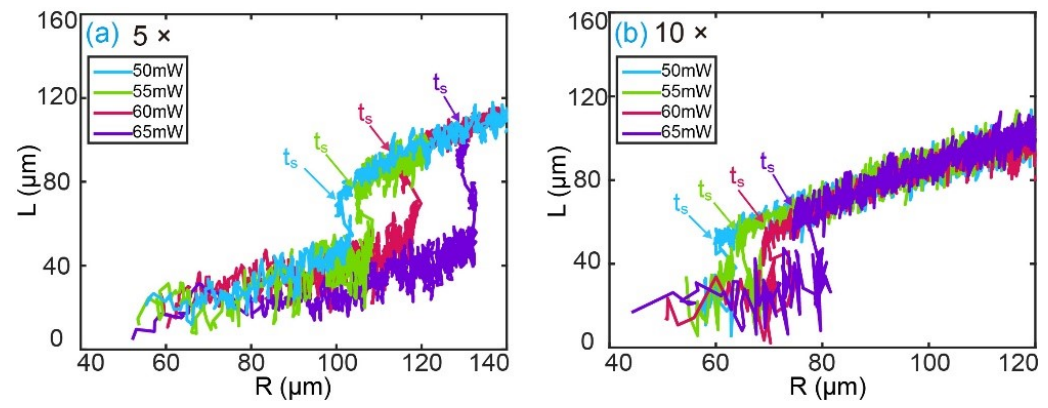

Figure 5.8: The footprint diameter $L$ of the bubbles versus bubble radius $R$ with different laser power values under $5 \times$ objective (a) and $10 \times$ objective (b), respectively.

\subsubsection{Comparison of the bubble shrinkage dynamics on a sub- strate with a gold layer and gold nanoparticle decorated substrate}

To confirm that the observed bubble shrinkage is not sample specific, we repeated the experiments on a substrate with a smooth gold layer immersed in a binary liquid with ethanol fraction $f_{e}=70 \%$. As shown in Figs. 5.9.(a) and (b), the contact angle of the water droplet on the bare gold substrate 
is $57.6^{\circ}$, which is much larger than the contact angle of water droplet on the gold nanoparticle decorated fused silica substrate. We also measured the roughness of the two surfaces through an atomic force microscope. Atomic force microscope (AFM) height images for the two samples are shown in Figs. 5.9.(a) (II) and 5.9(b) (II). The measured roughness values are $34.2 \mathrm{~nm}$ and $1.4 \mathrm{~nm}$ for the nanoparticles decorated substrate and the one with the gold layer, respectively.

The comparison of the bubble growth dynamics on the gold layer substrate and gold nanoparticle decorated substrate is shown in Fig. 5.9(c). Despite the large difference in surface roughness, the same sudden decrease in the bubble radius is observed. This means that the sudden bubble shrinkage process is a robust result and not sample-specific.
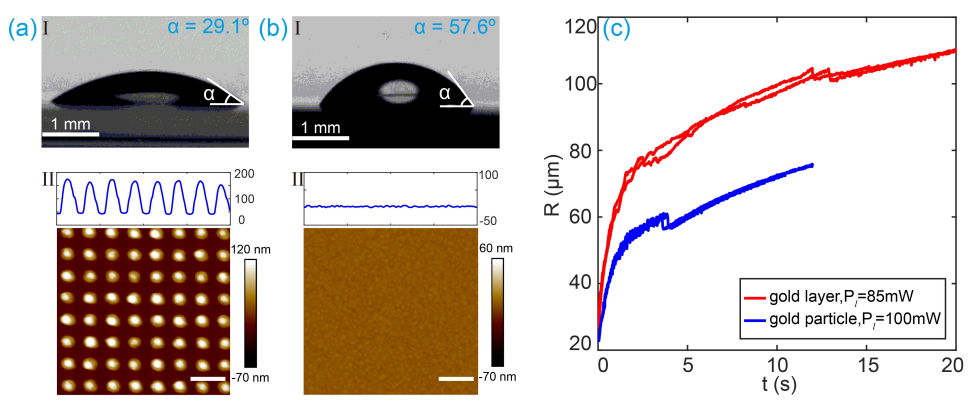

Figure 5.9: Contact angle of a water droplet and roughness measurement (via AFM height image measurement) for substrates with gold nanoparticle array (a) and a gold layer (b). (Scale bars in AFM height images: $200 \mathrm{~nm}$ ) The static contact angles of the water droplets on the two substrates are $29.1^{\circ}$ and $57.6^{\circ}$, respectively. (c) The bubble growth dynamics on the gold nanoparticle decorated substrate at $P_{\ell}=100 \mathrm{~mW}$ and on the gold layer covered substrate at $P_{\ell}=85 \mathrm{~mW}$ in a binary liquid with $f_{e}=70 \%$. 


\section{Chapter 6}

\section{Solvent exchange in a Hele-Shaw cell: universality of surface nanodroplet nucleation 1}

Solvent exchange (also called solvent shifting or Ouzo effect) is a generally used bottom-up process to mass-produce nanoscale droplets. In this process, a good solvent for some oil is displaced by a poor one, leading to oil nanodroplet nucleation and subsequent growth. Here we perform this process on a hydrophobic substrate so that sessile droplets - so-called surface nanodroplets - develop, following the work of Zhang et al. [Zhang, X.; Lu, Z.; Tan, H.; Bao, L.; He, Y.; Sun, C.; Lohse, D. Proc. Natl. Acad. Sci. U. S. A. 2015, 122, 9253-9257]. In contrast to what was done in that paper, we chose a very well controlled Hele-Shaw geometry with negligible gravitational effects, injecting the poor solvent in the center of the Hele-Shaw cell, and characterize the emerging nanodroplets as function of radial distance and flow rates. We find that the mean droplet volume per area $\langle V o l\rangle_{\text {area }}$ strongly depends on the local Peclet number $P e$ and follows an universal scaling law $\langle V o l\rangle_{\text {area }} \sim P e^{3 / 4}$. Moreover, the probability distribution function of the droplet volume strongly depends on the local $\mathrm{Pe}$ as well, regardless of the flow rates and radial distance, giving strong support to the theoretical model of the solvent exchange

\footnotetext{
${ }^{1}$ Published as: Binglin Zeng, Yuliang Wang, Xuehua Zhang, and Detlef Lohse, Solvent exchange in a Hele-Shaw cell: universality of surface nanodroplet nucleation, J. Phys. Chem. C 123, 5571-5577 (2019).
} 
process developed in Zhang et al's work. 


\subsection{Introduction}

The solvent shifting process - also called solvent exchange - is a simple and generic approach for mass-producing droplets or bubbles at solid-liquid interfaces. The droplet or bubble are only several tens to hundreds of nanometers in height, or a few femtoliters in volume [156,163 167]. In this process, a good solvent is replaced by a poor solvent, leading to nanodroplets or nanobubble nucleation and subsequent growth on the substrate. The approach has several potential applications, such that it can be used for liquid-liquid microextraction, diagnosis, drug production, extraordinary focusing, micromanufacture and among many others [38, 39, 41, 168 177]. When applied to oil dissolved in a good solvent, the solvent exchange process has the capability of massproducing surface nanodroplets of oil on substrates in one step [43, 49, 178 181].

In ref. [43] the solvent exchange process was performed in a linear channel, with the flow rate $Q$ and the channel height $h$ as control parameters. The nanodroplet generation for seven different flow rates and three different channel heights between $h=0.33 \mathrm{~mm}$ and $h=2.21 \mathrm{~mm}$ was analysed. The main result was that the experimentally found mean droplet volume was consistent with the theoretical results derived in the same paper, namely that the areaaveraged volume of the droplet $\langle V o l\rangle_{\text {area }} \sim h^{3} \mathrm{Pe}^{3 / 4}$, where area-averaged volume $\langle V o l\rangle_{\text {area }}$ is defined by the total volume of droplets over a unit surface area, $P e=Q /(w D)$ is the Peclet number, defined by the flow rate, the width of the channel $w=A / h$ (where $A$ is the rectangular channel cross section), and the mass diffusivity $D$ of the oil, see Fig. $2 \mathrm{G}$ of that paper.

However, for the larger channel heights analyzed in that paper, major convective effects sets in, due to the density difference between the two solvents, leading to considerable inhomogeneities in droplet sizes. Indeed, the role of gravity in the solvent exchange process in such thick channels could later be confirmed in ref. [48].

The aim of this present paper therefore is to go to a different geometry, namely to a Hele-Shaw cell: a channel formed by two closely spaced parallel glass plates. The employed Hele-Shaw cell possesses a much smaller cell height of $h=100 \mu m$, implying an Archimedes number $A r=g h^{3} \Delta \rho /\left(\nu^{2} \rho\right)$ (see ref. [48]) of $A r=0.61$, where $g=9.81 \mathrm{~m} / \mathrm{s}^{2}$ is the gravitational acceleration, $\nu=10^{-6} \mathrm{~m}^{2} / \mathrm{s}$ is the kinematic viscosity, $\rho=1000 \mathrm{~kg} / \mathrm{m}^{3}$ is the density of the fluid for injection (deionized water, in this case), and $\Delta \rho=62 \mathrm{~kg} / \mathrm{m}^{3}$ is the density difference between a solution to be repelled from the Hele-Shaw cell (water-ethanol solution with the ethanol concentration of $30 \mathrm{vol} \%$, in 

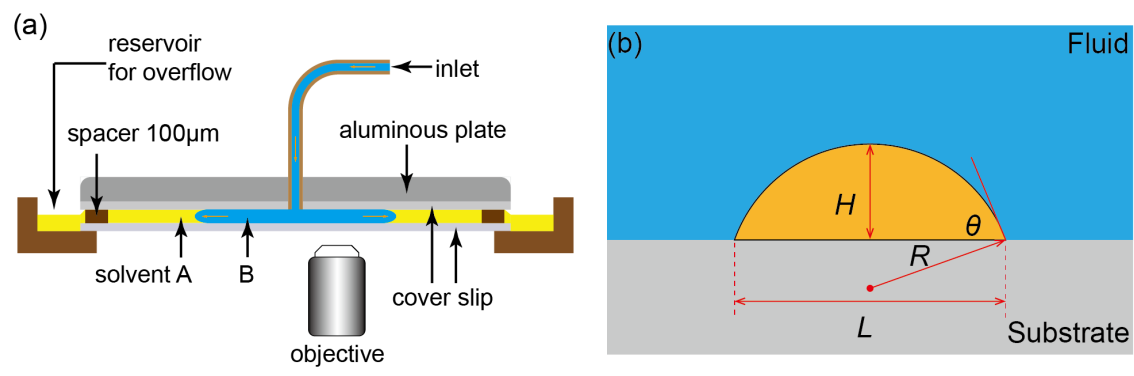

Figure 6.1: (a) Schematic drawings of the employed Hele-Shaw cell. The cell consists of a bottom cover slip window, a top cover slip attached to an aluminous plate, a spacer of $100 \mu \mathrm{m}$ in height, an inlet for liquid and a reservoir for overflow. During experiment, the liquid injected through the inlet will flow outwards in radial direction and is observed from the bottom. (b) Schematic diagram of a nanodroplet, where $R, \theta, H$, and $L$ are the radius of curvature, the contact angle, the height, and the footprint diameter of the droplet.

this case) and the fluid for injection. For $A r<1$, gravitational effects can be neglected. The Hele-Shaw geometry has the additional advantage that the flow rate now depends on the radial distance $r$ from the point of flow injection, allowing for continuous variation of the local flow velocity, which again leads to the continuous tuning of the Peclet number, which is again the non-dimensionalized flow rate, here given by

$$
P e=\frac{Q}{2 \pi r D}
$$

As a result, the correlation of nanodroplet formation with Peclet number can be easily investigated. The main question which arises is: Does the relationship $\langle V o l\rangle_{\text {area }} \sim h^{3} \mathrm{Pe}^{3 / 4}$ also locally hold in the Hele-Shaw geometry and is thus universal?

Figure 6.1 shows a sketch of the geometry and the employed notations for the nanodroplets. With this setup, we want to test the fluid dynamical theory of solvent exchange (which can straightforwardly be generalized to the present circular geometry) developed in reference [43].

The paper is organized as follows: Section 2 gives the details on the employed method and how the data were collected. Section 3 shows the results, followed by a discussion and the conclusions (section 4 ). 


\subsection{Experiment}

\subsubsection{Sample preparation}

A circular glass cover slip (GOLO, China) with a diameter of $50 \mathrm{~mm}$ was used as the substrate for droplet nucleation and bottom window for observation in the Hele-Shaw cell. The substrate was hydrophobilized by PVDFHFP (poly(vinylidene fluoride-co-hexafluoropropylene), $\mathrm{Mw}=400000$, SigmaAldrich, USA). To do so, the cover slip was first cleaned in a sonication bath of piranha solution $\left(70 \% \mathrm{H}_{2} \mathrm{SO}_{4}-30 \% \mathrm{H}_{2} \mathrm{O}_{2}\right.$ solution) for $30 \mathrm{~min}$, followed by the sonication bath of acetone and ethanol for $30 \mathrm{~min}$, then in deionized water 3 times for $5 \mathrm{~min}$. After that, the sample was dried by nitrogen gas. The dried cover slip was then immersed in a $3 \mu \mathrm{L}$ PVDF-HFP in a petri dish. The petri dish remained in an oven at $150{ }^{\circ} \mathrm{C}$ for $12 \mathrm{~h}$. Eventually, a hydrophobic glass substrate coated with PVDF-HFP was obtained. The measured static contact angle of water on the obtained PVDF-HFP hydrophobic surface is $110^{\circ}$.

\subsubsection{Formation of nanodroplets through solvent exchange in the Hele-Shaw geometry}

Nanodroplets of trans-anethole (4-Propenylanisole, trans-1-Methoxy-4-(1-propenyl) benzene, Solarbio, USA) were produced on the obtained hydrophobic substrate in a Hele-Shaw cell through solvent exchange. As shown in Fig. 6.1. (a), the top cover slip and the bottom cover slip window form a disk-shaped channel with a height $h=100 \mu \mathrm{m}$ and a diameter $d=50 \mathrm{~mm}$. Unlike the liquid cell applied in ref. [43], the inlet of the Hele-Shaw cell is in the center of the cell. The size and area density of the droplets are influenced by the solution composition for the solvent exchange [49, 182]. In our experiments, two solutions, solvent A and solvent B, were prepared for solvent exchange. Solvent $\mathrm{A}$ is an aqueous ethanol solution with the ethanol (analytically pure, $99.8 \%$, Aladdin, China) concentration of 30 vol\%. The solution was saturated with trans-anethole, which was labeled by a fluorescence dye perylene (Klamar-reagent, China). Solvent A serves as good solvent for trans-anethole with saturation concentration of $0.55 \mathrm{wt} \%$. Solvent B is deionized (DI) water, which serves as poor solvent for the trans-anethole. Before the experiments, solvent B was saturated with trans-anethole as well. Such solution composition produced desirable size and density of droplets, suitable for optical images and data analysis. During the solvent exchange process, $200 \mu \mathrm{L}$ solution A was displaced by $2 \mathrm{~mL}$ solution B. The injection of solution B was performed 
by a syringe pump at three different flow rates of $Q_{1}=1000, Q_{2}=500$, and $Q_{3}=300 \mu \mathrm{L} / \mathrm{min}$.

\subsubsection{Characterization of nanodroplets}

After the tran-anethole droplets had nucleated on the hydrophobic glass substrate, they were characterized by a reflecting fluorescence microscope (IX71, Olympus, Japan). Two objectives $(4 \times$ and $20 \times$ ) were used in the imaging of the nucleated droplets. The images taken with the $20 \times$ objective were used for morphological analysis, while the ones taken with the $4 \times$ objective were used to determine the radial distance $r$ for each selected area in the optical images taken with the $20 \times$ objective. All the optical images $(20 \times)$ were analyzed using a home designed image segmentation algorithm for the optimized extraction of the droplet footprint diameters. For details of the algorithm, readers are referred to our previous publications [62, 65, 67, 183].

For each of the three flow rates, the nucleated nanodroplets with different radial distance $r$ were captured. The number of the captured nanodroplets are 18332, 14403, and 19990 for flow rates of $1000 \mu \mathrm{L} / \mathrm{min}, 500 \mu \mathrm{L} / \mathrm{min}$, and $300 \mu \mathrm{L} / \mathrm{min}$, respectively. For each $Q$, the captured droplets were binned in concentric circular bands with a band width $\Delta r=100 \mu \mathrm{m}$.

To extract volumes of the nucleated nanodroplets, a tapping mode atomic force microscopy (TM-AFM) (Resolve, Bruker, USA) was also applied to get high-resolution three dimensional (3D) images of the nucleated droplets, after the growth of the oil droplets was already finished. The nucleated nanodroplets remained in solvent B and were imaged in the liquid mode of TM-AFM. For the duration of the operational time, we did not observed any temporal evolution in the droplet lateral size. The obtained AFM images were analyzed with a home-designed Matlab program to extract the height, width and contact angle of the droplets, as reported in our previous work [184]. After that, the volumes of the nanodroplet can be obtained.

Since the number of the captured nanodroplets is huge, it is impractical to measure the volumes of all individual nanodroplets with AFM. Therefore, in this study, only a few nanodroplets with different footprint diameters were imaged with TM-AFM, in order to establish the dependence of the nanodroplet height $H$ and contact angle on its footprint diameter. The height of droplets $H$ was measured for droplets with different footprint diameter $L$, as shown in Fig. 6.3(b). From the $H(L)$ relationship, we obtained the $\theta(L)$ dependence, as shown in the dotted blue curve, which provides the basis for estimation of the contact angle for droplets with different $L$ determined from the optical 
images. This dependence was further used to obtain the volume for each captured nanodroplet in the optical images from the footprint diameter.

\subsubsection{Fluid dynamics theory of solvent exchange}

The scaling law between the final area-averaged volume of the droplet $\langle\mathrm{Vol}\rangle_{\text {area }}$ and the Peclet number $P e$ was established in ref. [43]. When gravitational effect in solvent exchange process can be ignored (i.e., for small Archimedes number), the final area-averaged droplet volume scales as:

$$
\langle V o l\rangle_{\text {area }} \sim h^{3}\left(\frac{C_{\text {sat }}}{\rho_{\text {oil }}}\right)^{3 / 2}\left(\frac{C_{\infty}}{C_{\text {sat }}}-1\right) P e^{3 / 4}
$$

where $C_{s a t}$ and $C_{\infty}$ are the saturation concentrations of oil in the poor solvent and the actual oil concentration, respectively, and $\rho_{\text {oil }}$ is the oil density. This fluid dynamics theory of solvent exchange will be further experimentally validated in this paper.

\subsection{Results and Discussion}

After solvent exchange, nucleated nanodroplets were attached to the bottom cover slip window. Figure 6.2(a) shows an fluorescence image obtained by aligning different images taken with the $4 \times$ objective at the flow rate of $Q=$ $1000 \mu \mathrm{L} / \mathrm{min}$. Three examples of images taken with a higher magnification objective of $20 \times$ are shown in Fig. 6.2(b) for the three selected areas in Fig. 6.2(a). From the images, one can clearly see that the droplet size decreases with increasing distance $r$ from the center. Figure 6.2(c) shows an enlarged image for an area selected by the dotted green box in Fig. 6.2(a). One can see that the droplets in the area are lined up along the flow direction (as guided by a dotted orange arrow) and exhibits nonuniform size distributions. This will be discussed later in this section.

Figure 6.3(a) shows a representative AFM image of the trans-anethole droplets scanned in solvent B on the bottom substrate. From the AFM images, the height and the contact angle for the nanodroplets were extracted, as shown in Fig. 6.3(b). One can see that the droplet height $H$ roughly linearly increases from about $0.2 \mu \mathrm{m}$ to $1.3 \mu \mathrm{m}$ as $L$ increases from about $1.5 \mu \mathrm{m}$ to $11 \mu \mathrm{m}$. The contact angle $\theta$ basically remains constant at $27^{\circ}$ after $L$ is larger than $3 \mu \mathrm{m}$. The aspect ratios of the nucleated droplets are consistent with 


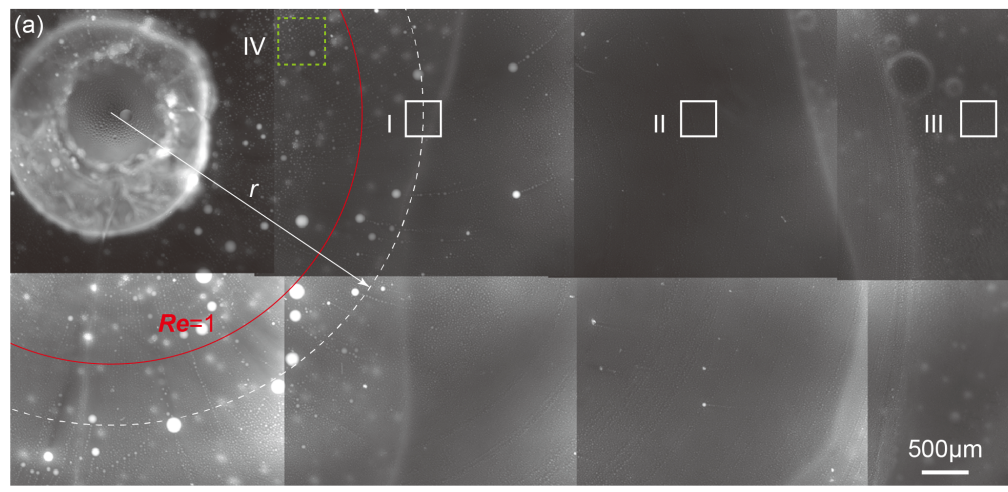

(b)
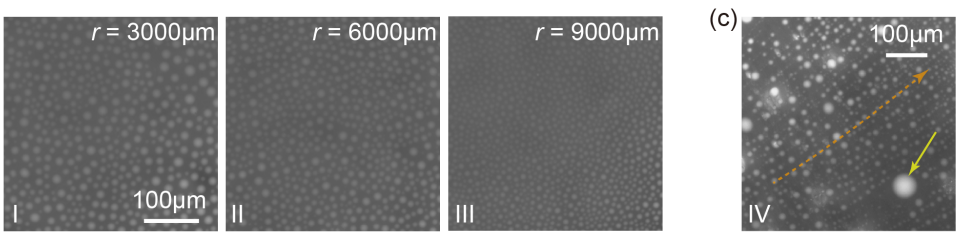

Figure 6.2: Morphological characterization of the nucleated nanodroplets in the Hele-Shaw geometry: (a) An optical microscope image obtained by aligning different images taken at different radial distance $r(4 \times$ objective) for $Q=$ $1000 \mu \mathrm{L} / \mathrm{min}$. (b) Three example images taken with the $20 \times$ objectives at the three selected positions in white boxes of I, II, and III in (a). (c) An enlarged image for an area selected by a dotted green box IV in (a).

the results reported in ref. [43, 185] for the same combination of solvents and solutes.

With the developed dependence between $H, \theta$, and $L$, the droplet volumes $V o l$ for individual captured droplets in the optical microscope images were obtained. After that, the mean droplet volume per area $\langle V o l\rangle$ area was further calculated, as shown in Fig. 6.4(a). For all the three flow rates, $\langle\text { Vol }\rangle_{\text {area }}$ decreases with increasing $r$. Moreover, for the same $r,\langle V o l\rangle_{\text {area }}$ increases with increasing flow rates. Most importantly, regardless of the flow rates, all the three curves are well superposed on each other in the plot of $\langle\mathrm{Vol}\rangle_{\text {area }}$ versus Peclet number $P e$, as shown in Fig. 6.4(b), especially for lower $P e$ value (corresponding to larger distance $r$ from the inlet).

The double logarithmic plots of $\langle V o l\rangle_{a r e a}$ versus the radial distance $r$ and $v s$ the Peclet number $P e$ are shown in Figs. 6.4(c),(d). It is clear that $\langle\mathrm{Vol}\rangle_{\text {area }}$ shows a $-3 / 4$ and $3 / 4$ power law dependence on $r$ and $P e$, respectively. Most 
(a)

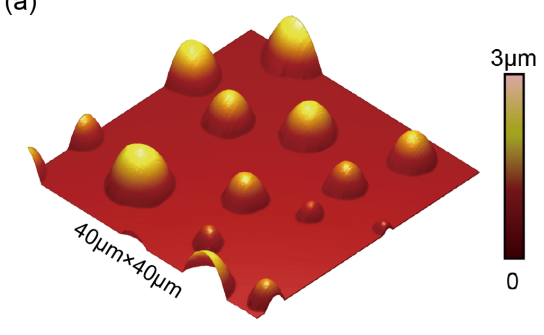

(b)

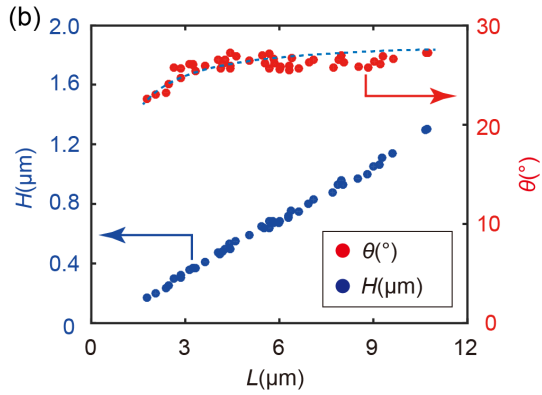

Figure 6.3: (a) A TM-AFM image of the nucleated droplets, providing their high-resolution 3D morphological characterization. (b) The height $H$ and the contact angle $\theta$ as functions of the footprint diameter $L$ of the droplets. The dependence were further used to obtain $H$ and $\theta$ for nanodroplets in the optical images.

importantly, three curves in Fig. 6.4 (d) collapse on one universal curve. These results are consistent with the theoretical prediction (Eq. 6.2) and provide strong support to the model.

In Figs. 6.4(c), (d), for smaller $r$ or larger $P e$, the data points for $Q=1000$ $\mu \mathrm{L} / \mathrm{min}$ start to deviate from the $-3 / 4$ or $3 / 4$ power law scaling lines. The deviation is likely due to the higher Reynolds number corresponding to smaller $r$. In the Hele-Shaw cell, the Reynolds number $R e$ at the radial distance $r$ is given by

$$
R e=\frac{Q}{2 \pi r \nu}
$$

where $\nu$ is the kinematic viscosity of the solution (here $\nu=10^{-6} \mathrm{~m}^{2} / \mathrm{s}$ for water). In Fig. 6.4.(c), the red, green, and blue vertical dashed lines correspond to $R e=1$ for the flow rates of $1000 \mu \mathrm{L} / \mathrm{min}, 500 \mu \mathrm{L} / \mathrm{min}$, and $300 \mu \mathrm{L} / \mathrm{min}$, respectively. In Fig. 6.4(d), a dashed vertical line was also drown at the position of $R e=1$. One can see that the deviations mainly occur in the area where the Reynolds number $R e>1$. Indeed, at higher Reynolds numbers, the flow becomes dominated by inertia effects and the laminar theory of ref [43 would require extensions. Namely, vortical flow structures may occur, leading to a less organized flow pattern. This may lead to advection of nucleated droplets.

For that case $R e>1$, droplets indeed can move outwards along the radial direction of the Hele-Shaw cell. This can be seen from Fig. 6.2(c). It is an enlarged image for the area highlighted by the dotted green box in the region 

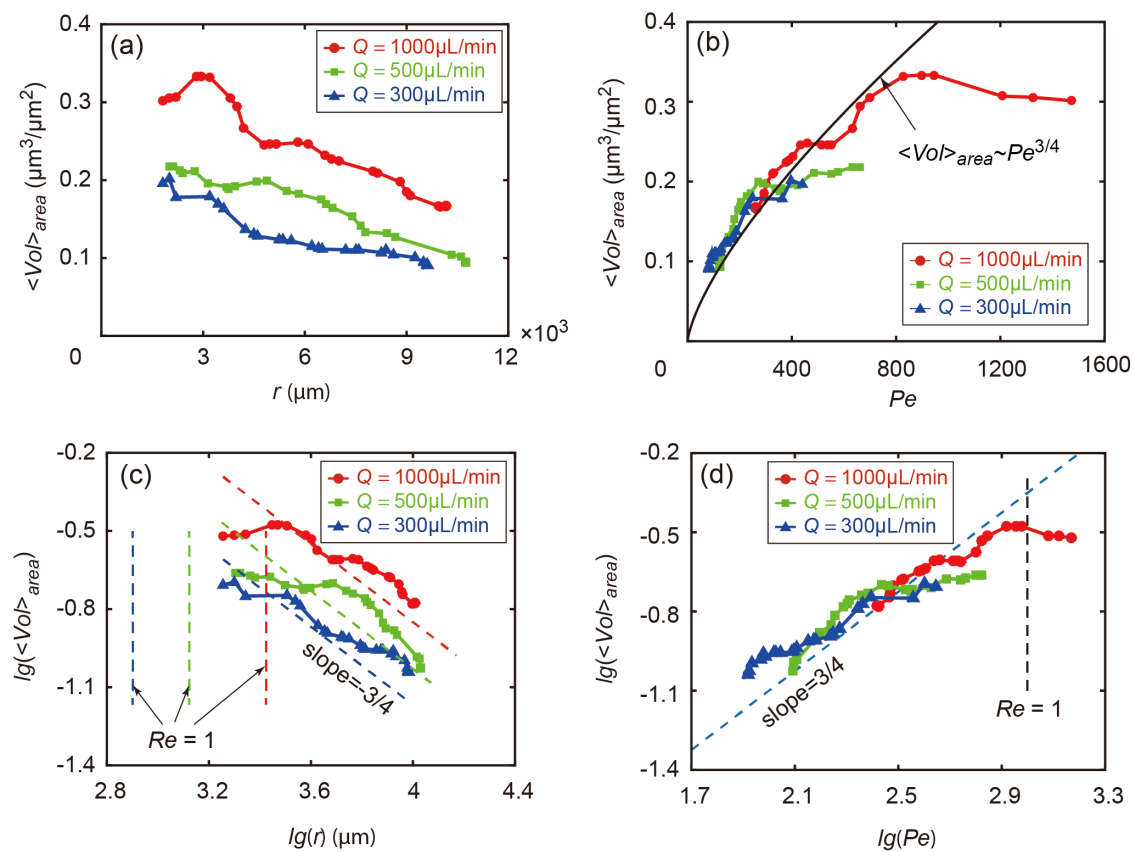

Figure 6.4: (a, c) Linear and double-logarithmic scale plots of the mean volume per area $\langle\mathrm{Vol}\rangle_{\text {area }}$ of the nucleated nanodroplets as a function of radial distance $r$ from the flow inlet for the three different flow rates $Q$ of 1000 $\mu \mathrm{L} / \mathrm{min}, 500 \mu \mathrm{L} / \mathrm{min}, 300 \mu \mathrm{L} / \mathrm{min}$ (top to bottom). (b, d) Linear and doublelogarithmic scale plots of $\langle V o l\rangle_{\text {area }}$ as a function of the dimensionless form of flow velocity - Peclet number $P e$ for different $Q$. All three curves collapse on a curve with a slope of $3 / 4$ (shown as straight line) in the log-log plot, indicating an universal scaling law of $\langle V o l\rangle_{\text {area }} \sim P e^{3 / 4}$.

corresponding to $R e>1$, which is defined by a red circle in Fig. 6.2 (a). In the image, one can see that the droplets are mostly lined up along the radial direction, which is clearly different from that shown in Fig. 6.2(b). The advected droplets can merge with other surface droplets on their paths, leading to an increased size of droplets, as pointed at by the arrow in Fig. 6.2(c). Meanwhile, the strong outward motion of droplets unavoidably leads to a reduced average volume $\langle V o l\rangle_{\text {area }}$ close to the center flow inlet. As a result, in the region with $R e>1,\left\langle V_{o l}\right\rangle_{\text {area }}$ is smaller than what is predicted by the model Eq. (6.2) of reference [43] and the experimental results deviate from the power law line, as shown in Figs. 6.4 (c),(d). 

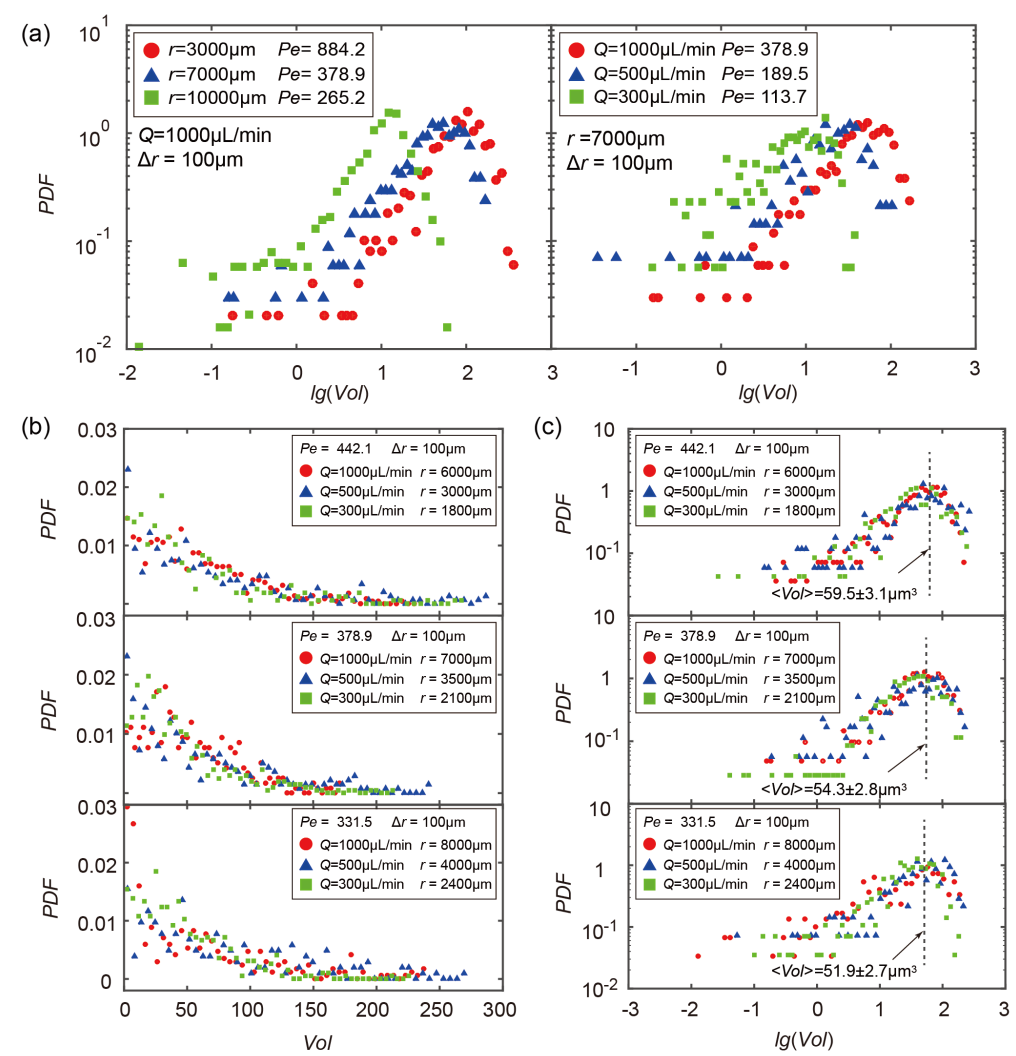

Figure 6.5: Droplet volume PDFs for various Peclet numbers Pe. (a) Left: PDF of the droplet volume for three different distances $r$ from the point of flow injection at the same flow rate $Q$ of $1000 \mu \mathrm{L} / \mathrm{min}$. Right: PDFs of the droplet volume at the same radial distance $r$ for three different flow rate $Q$. For both cases, the PDFs shift rightwards with increasing $P e$. (b, c) Comparison of the PDFs of droplet volume in linear (b) and double logarithmic (c) plots for droplets nucleated in the areas with the same local $P e$ but different combinations of $Q$ and $r$. One can conclude that the PDFs of the droplet volume strongly depend on the local $P e$, regardless of $Q$ and $r$.

Compared to the result reported in ref. [43] (Fig. 2G, therein), the result shown in Fig. 6.2(d) provide a higher consistency among the experiments with different flow rates. This is attributed to the smaller cell height of $h$ $=100 \mu \mathrm{m}$, which eliminates gravitational effects (i.e., $A r<1$ ). The results 
thus give strong support to our previously developed fluid dynamics model of solvent exchange, namely, that the volume of the nucleated droplets strongly depends on the dimensionless flow velocity, perfectly following the relationship $\langle\text { Vol }\rangle_{\text {area }} \sim h^{3} \mathrm{Pe}^{3 / 4}$.

After having shown the universality of the $3 / 4$ power law dependence between $\langle V o l\rangle_{\text {area }}$ and $P e$, one also wonders on the universality of the probability distribution function (PDF) of the droplet volumes, i.e., on the dependence of the PDFs on $P e$. To answer this question, the distribution of the droplet volume was calculated. Figure 6.5 (a) shows the PDF of the droplet volume at different $r$ for the flow rate $Q=1000 \mu \mathrm{L} / \mathrm{min}$ (left figure) and at the same radial distance $r=7000 \mu \mathrm{m}$ for different flow rates (right figure). In the left figure, the PDF of droplet volumes shifts rightwards with decreasing $r$. Similarly, the PDF shifts rightwards with increasing $Q$ in the right figure. Both figures of course reflect that the mean droplet volume increases with $P e$.

Since $P e$ is continuously varying in the Hele-Shaw cell, this gives us the freedom to select a particular $P e$ value for fixed flow rate. This allows us to compare the distribution of the droplet volume with the same $P e$ value, but different $Q$. For the three different flow rates, three different local $P e$ values of $P e=442.1,378.9$, and 331.5 were selected. This corresponds to nine areas in total, three for each of flow rate. The linear and double-logarithmic plots of the PDF of the droplet volumes for the three selected $P e$ values are shown in Figs. 6.5 (b) and (c), respectively. Remarkably, it shows that the PDFs of the droplet volume have an universal dependence on the Peclet number, regardless of the radial distances $r$ and flow rates $Q$.

\subsection{Conclusions}

In summary, we experimentally investigated the formation of surface nanodroplets by solvent exchange under a well controlled flow conditions. Compared to the rectangular cross section channels used in one of our previous study [43], a Hele-Shaw cell with a cell height of $100 \mu \mathrm{m}$ was employed. In the new setup, gravitational effects can be negligible. Moreover, the Hele Shaw setup easily allows the continuous tuning of the dimensionless flow velocity - namely the Peclet number Pe. By combining a fluorescence optical microscope and an AFM, the height, contact angle, footprint diameter, and volume of surface nanodroplets were extracted for a huge amount of nanodroplets under three different flow rates. The results reveal the underlying mechanism governing the droplet nucleation through solvent exchange. They show that 
not only the mean droplet volume, but also the PDF of droplet volumes universally depends on the local $P e$ number. Although the size of the nucleated droplets changes with radial distance $r$ and flow rate $Q$, the mean droplet volume per area $\langle V o l\rangle_{\text {area }}$ shows a universal 3/4 power law dependence on $P e$. This is in a good agreement with the model developed in our previous work [43]. Moreover, further investigation shows that the PDFs of the droplet volume also follow an universal dependence on the local $P e$, regardless of the radial distance $r$ and the employed flow rate $Q$. The revealed dependencies provide an important guideline for the control of the flow conditions in the mass-production of surface nanodroplets, which is very relevant for various applications, such as in diagnostics, liquid-liquid microextraction, drug production, or food-processing. 


\section{Chapter 7}

\section{Formation of polystyrene microlenses via transient droplets from the ouzo effect for enhanced optical imaging 1}

Lens-shaped microstructures on solid surfaces are important for a variety of applications, such as enhanced water harvesting, super-resolution imaging, and antireflection. Here the formation of polymeric microlenses with tunable contact angles based on an Ouzo effect is reported. In this process, water is added into a binary toluene/ethanol solution in contact with a polystyrene (PS) thin film. The dilution by water leads to spontaneous formation of toluene microdroplets due to the reduced solubility of toluene in the ternary liquid mixture (i.e., the Ouzo effect). PS in the thin film is dissolved into the toluene droplets. However, the droplets containing PS and toluene are not stable against dissolution, and eventually toluene dissolved into the surrounding ternary mixture. PS in the droplets is left on the substrate, forming microlenses on the supporting glass substrate. The size and density of PS lenses are influenced by PS film thickness and toluene concentration in the surrounding liquid. The contact angle of PS microlens could be varied through a thermal reshaping method. As demonstration for potential applications, the results show that

\footnotetext{
${ }^{1}$ Published as: Yuliang Wang, ${ }^{*}$ Binglin Zeng, Yong Zhao, Shuai Li, and Xuehua Zhang, ${ }^{*}$ Formation of polystyrene microlenses via transient droplets from the ouzo effect for enhanced optical imaging, J. Phys. Chem. C 123, 14327-14337 (2019). Experimental work is done by Zeng.
} 
the as-prepared microlenses can improve the spatial resolution of a standard upright optical microscope. 


\subsection{Introduction}

Lens-shaped microstructures on solid surfaces have numerous applications, such as surface antireflective coatings [170], bioimaging 186, data storage [187], optical lithography [188], ultraprecision positioning [189, 190], optothermal conversion [40] and surface-enhanced Raman scattering [191]. Remarkably, recently it has been demonstrated that microlenses are able to overcome the diffraction limit and hence improve the resolution of optical imaging systems $[192$ 194]. Microlenses can be fabricated either individually or in batches. Individual lenses can be fabricated through crystal growth [192], thermal reflow [195] and suspension polymerization [193]. To increase the fabrication efficiency, methods are developed to produce microlenses in batches, such as dewetting of polymer films [196 200], inkjet printing [188, 201], surface wrinkling [202], laser swelling [203] and photopolymerization of monomer precursor droplets [42, 43, 170, 204]. In the surface wrinkling and laser swelling methods, the polymeric films are deformed (wrinkling and swelling) to generate microlenses [202,203]. It is difficult to guarantee a regular spherical cap shape. In the inkjet printing method, the solvent droplets with dissolved microlens materials are first transferred onto sample surfaces. Microlenses are formed after the solvent is evaporated. However, the evaporation of liquid leads to an irregular shape of the fabricated microlenses [188]. To improve the sphericity of the fabricated microlenses, photopolymerization can be directly used to convert the monomer precursor droplets into solid microlenses by a process which is termed as the Ouzo effect [205, 206].

Contact angle is an important parameter in the preparation of microlenses. In previous work, the wettability of the substrates or the surrounding medium could be varied to adjust the contact angles of microlenses fabricated by polymerization of precursor surface nanodroplets [207]. In these studies, the droplets were formed by the solvent exchange, a process that relies on the Ouzo effect. The contact angles of the obtained nano/microlenses were usually from $9^{\circ}$ to $90^{\circ}$ due to the low contact angle of stable precursor droplets on the surface. By a complementary method based on the polymer film dewetting, the microlens size was controlled by polyer film thickness, and the contact angle could be tuned through controlling the reaction time [199]. It is also reported that the polymeric lenses will spread out after annealing them above their glass transition temperature [196, 198]. Inspired by the annealing method in the dewetting-based microlens fabrication method, contact angles of microlenses are supposed to be continuously tuned by control temperature. 
This conjecture will be later verified in this work.

In this study, we propose a novel process for producing microlenses with well-controlled contact angles. The process is based on the Ouzo effect where toluene microdroplets spontaneously form in a ternary mixture of ethanolwater-toluene. The surface toluene droplets dissolve a PS film,resulting in the formation of toluene-PS droplets. The transient toluene droplets redissolved into the surrounding liquid, and the toluene-PS droplets were converted into PS lenses. The mechanism of lens fabrication will be explored, together with the approaches of systematically tuning the size, density, as well as contact angles of the microlenses. Compared to the polymerization of precursor surface nanodroplets, our method can fabricate microlenses with much higher contact angles from $50^{\circ}$ to $125^{\circ}$. As a result, a larger magnification factor can be obtained with the microlenses which have larger contact angles for enhanced optical imaging [193]. Moreover, both size and density of microlens can be tuned by adjusting polystyrene thickness and toluene concentration, offering controllability of microlenses with respect to their sizes and densities. We further demonstrated that the microlenses with the well-defined sphericalcap-like shape can be used to achieve enhanced imaging performance in an optical microscope.

\subsection{Experimental section}

\subsubsection{Preparation of PS films}

Glass slides of $1 \mathrm{~cm} \times 1 \mathrm{~cm}$ were taken as substrates. They were first cleaned in sonication bath of acetone and ethanol for $30 \mathrm{~min}$, followed by the $5 \mathrm{~min}$ sonication bath of deionized water three times. To get PS films with different thicknesses, PS particles (molecular weight 350000, Sigma-Aldrich) were dissolved into toluene with different concentrations of $0.25 \%, 0.5 \%, 1 \%, 2 \%, 3 \%$, and $5 \%$ (weight). After that, PS/toluene solution was spin-coated onto glass slides at speeds of 1500, 2000, 2500, 3000, 3000, and $3000 \mathrm{rpm}$, respectively. After that, the PS films were put into an oven (DZF, LICHEN, China) at 40 ${ }^{\circ} \mathrm{C}$ for $4 \mathrm{~h}$ to remove the remaining toluene.

To get the thickness of the PS films, atomic force microscopy (AFM) tips were first used to scratch PS films. The scratched PS films were then scanned using a tapping mode AFM. The PS film thickness was obtained through crosssectional profiles of the AFM height images containing the scratches. The measured PS film thickness is 18.0, 38.0, 77.0, 102.0, 179.0, and $234.0 \mathrm{~nm}$ for 
PS concentration of $0.25 \%, 0.5 \%, 1 \%, 2 \%, 3 \%$, and $5 \%$, respectively.

\subsubsection{Fabrication of PS Lenses}

PS films were first immersed in $200 \mu \mathrm{L}$ toluene/ethanol mixture solution in a Petri dish with a diameter of $2 \mathrm{~cm}$, and then $40 \mu \mathrm{L}$ deionized water was added into the mixture solution. After the PS lenses were formed, the glass substrates were then removed from the toluene/ethanol/water solution. After that, the substrates were rinsed with water and then sonicated in a water bath for $15 \mathrm{~min}$ to remove the weakly attached polymer particles. As shown in the solubility phase diagram (Fig. 7.1) of ethanol, toluene, and water, which was redrawn from Monica B. and Carlos M. (2003) [208], the initial component ratios in this study were located in the one phase region to ensure that the generated toluene droplets will gradually dissolve into the surrounding medium.

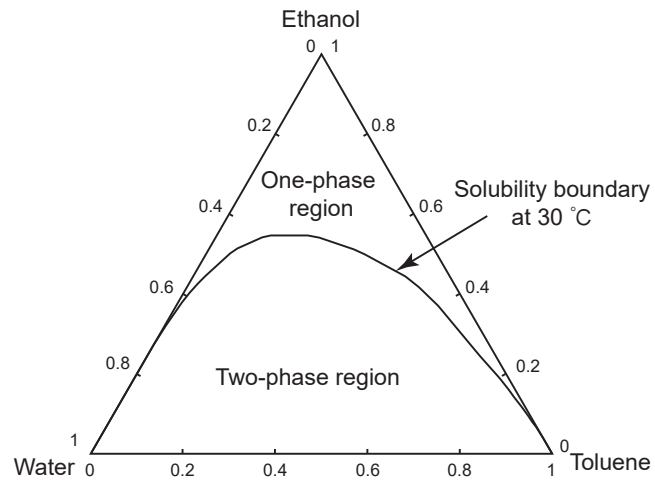

Figure 7.1: Solubility phase diagram of ethanol, toluene, and water, and the mutual miscibility curve at $30{ }^{\circ} \mathrm{C}$ (redrawn from Monica B. and Carlos M. (2003) [208]).

\subsubsection{Morphological Characterization of Microlenses}

An inverted optical microscope (IX 73, Olympus, Japan), an atomic force microscope (AFM, Resolve, Bruker, USA), and a scanning electronic microscope (SEM, proX, Phenom, Netherlands) were used to characterize microlenses. The optical microscope images are used to study the dynamic process of microlens formation, associated with a high-speed camera (EoSens 3CL, 
Mikrotron, Germany). For the optical images, a home-designed image segmentation program was applied for image processing [65-67]. From the program, the size and density of microlenses can be automatically extracted. Tapping mode AFM was used to characterize the microlenses in a three dimensional manner. From AFM images, the contact angle and volume of microlenses can be obtained. The side views SEM images were also used to compare the contact angle measurement results from AFM images.
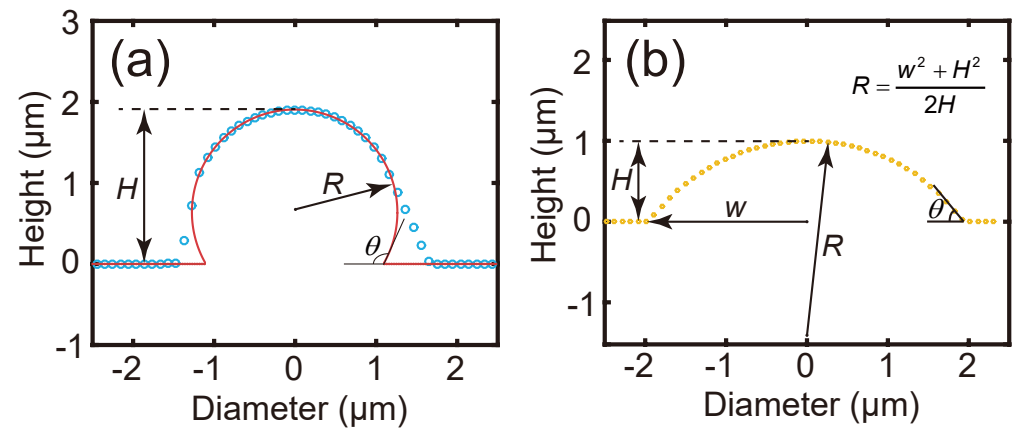

Figure 7.2: Cross-sectional profile of the lens from the AFM image. (a) When the lens contact angle is more than $90^{\circ}$, the data points above the center of the lens are used to get radius of curvature $\mathrm{R}$ and contact angle. (b) When the lens contact angle is less than $90^{\circ}$, the contact angle and radius of curvature of the lens can be directly obtained by fitting the entire cross-sectional profile as a circular arc.

Since the originally fabricated microlenses have contact angles over $90^{\circ}$, the region of microlenses close to the contact line with sample substrate cannot be scanned by AFM tips (Fig. 7.2(a)) [198]. Therefore, the portion of microlens profile above its center is fitted as circular shape. From the fitting result, radius of curvature $R$, the height $H$, and radius of contact line $w$ can be obtained. The contact angle $\theta$ and volume $V$ can then be given as

$$
\cos \theta=\frac{R-H}{R}
$$

and

$$
V=\frac{\pi}{3}(3 R-H) \times H^{2} .
$$

When the lens contact angle is less than $90^{\circ}$, the height $H$ and radius of contact line $\mathrm{w}$ can be directly obtained through the cross-sectional profiles, 
as shown in Fig. 7.2(b). The radius of curvature of microlenses can then be given as

$$
R=\frac{w^{2}+H^{2}}{2 H}
$$

\subsection{Results and discussion}

\subsubsection{Process of PS microlens formation}

The experimental procedure for the PS microlens fabrication is shown in Fig. 7.3. Figures 7.3(a)-(d) depict the schematic diagram of the lens fabrication process. The corresponding optical images are shown in Figs. 7.3(e)-(h). A smooth PS film on a glass substrate was first prepared using spin-coating method (Figs. 7.3(a) and (e)). The PS film was then immersed into a toluene/ethanol solution. After a drop of water was added into the solution, toluene droplets were then nucleated on the sample surface immediately (Figs. 7.3(b) and (f)). The nucleated toluene dissolved the PS film, leading to the formation of toluene/PS droplets (Figs. 7.3(c) and (g)). Meanwhile, toluene, ethanol, and water were gradually completely mixed in the bulk solution. Toluene in the toluene-PS droplets dissolved into bulk solution again, with the PS microlenses left on the glass substrate (Figs. 7.3(d) and (h)).

We found that droplets on the surface originated from three different sources. The first one is the direct nucleation of toluene droplets on the sample surface, as shown in Fig. 7.4(a). In this case, toluene droplets nucleated in liquid and attached on the PS film. The PS film was then dissolved by the droplets, and interfacial toluene/PS droplets were generated. After toluene was dissolved into solution, PS lenses were formed at the same positions where toluene/PS droplets were located. This kind of lenses is referred to as toluene droplet generated lenses. In the second case, PS film was ruptured, but no obvious toluene droplets were observed (Fig. 7.4(b)). PS film first became a network structure. Branches were then coalesced to form droplets. It is believed that a toluene film nucleated on the PS film induced the rupture of the PS film. This process is different from the dewetting-caused lens formation, as reported somewhere else [196].

In the dewetting-caused lens formation, the instability of polymer films is induced by high temperature or a specific solvent. After that, the films were turned into network structures and microlenses were eventually generated, which are referred to as toluene film generated lenses. However, in this study, 
(a)
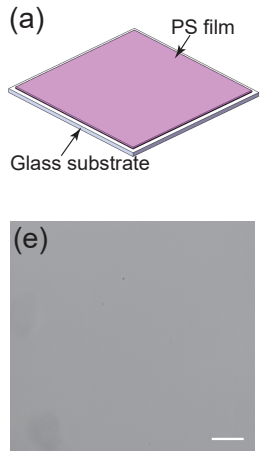

(b)
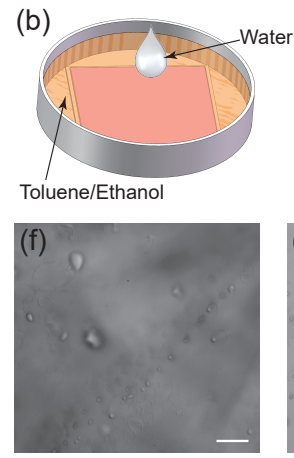

(c)

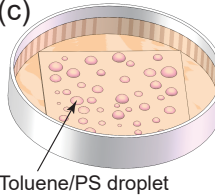

(g)

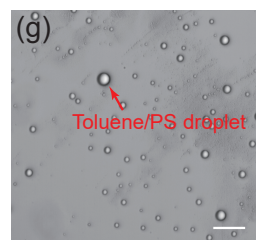

(d)

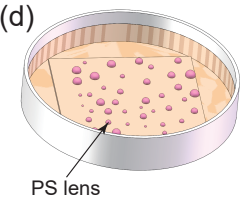

(h)

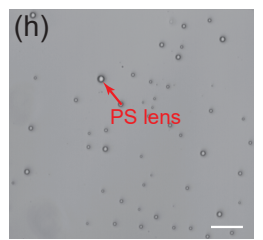

Figure 7.3: Illustration (a-d) and corresponding optical images (e-h) along with the formation of microlenses using the proposed transient toluene droplet approach. A PS film on a glass substrate $(\mathrm{a}, \mathrm{e})$ was first immersed into toluene/ethanol solution. A drop of water was then added into the solution $(b, f)$. Toluene/PS droplets were first generated onto glass surface $(c, g)$. After toluene dissolved back into the surrounding binary solution again, PS lenses were generated at the toluene/PS droplets position (d,h). (Scale bars: $50 \mu \mathrm{m}$.)

the droplets formed through the rupture of PS films are actually the mixture of toluene and PS. This can be seen from the fourth and fifth images in Fig. 7.4(b). After the droplet were formed, they gradually shrank because of the dissolution of toluene back to the bulk solution.

The third case is the nucleation of pure toluene droplets on the glass substrate (Fig. 7.4(c)). After PS film was dissolved on the surface, toluene droplets continued to nucleate on the glass surface. As a result, pure toluene droplets were generated. With time they dissolved again into surrounding solution and no lenses left.

\subsubsection{Morphology of PS microlenses.}

SEM and AFM scanning was used to characterize the obtained PS lenses. For a lens shown in Fig. 7.5(a), its SEM and AFM images are shown in Figs. 7.5(b) and (c), respectively. From the side view SEM image, a contact angle of $100^{\circ}$ was obtained. The measured contact angle is exact the same as the value measured from the cross-sectional profile of the AFM image (Fig. 7.5(d)). Since in SEM scanning only the lenses near the edge of sample substrates can be used for morphological characterization, AFM images were mainly applied for morphological characterization of the microlenses. 
(a) A toluene droplet induced PS lens

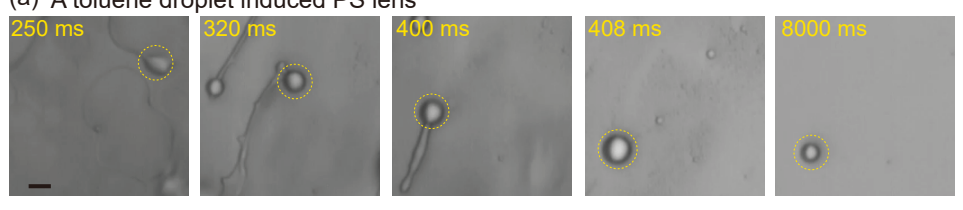

(b) Toluene film induced PS lens
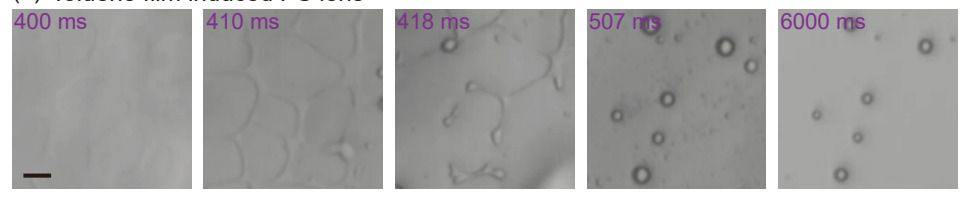

(c) Pure toluene droplet on glass substrate
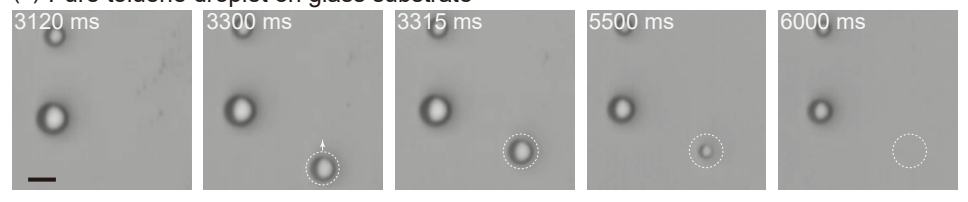

Figure 7.4: Three types of droplet nucleation on a sample surface after a drop of water was added into toluene/ethanol solution. (a) A nucleated toluene droplet attached PS film, dissolved the film, and formed a toluene/PS droplet, followed by the formation of a PS lens. (b) Toluene/PS droplets formation through PS film rupturing induced by a toluene film. The droplets were also eventually converted into PS lenses after the toluene was redissolved into the bulk solution. (c) A pure toluene droplet attached on the bare glass substrate and eventually disappeared again after the toluene was redissolved into the bulk solution. No lens was observed. The beginning of time in all the sequential images is the moment when the water was added (scale bars: $10 \mu \mathrm{m}$.)

Experimental results show that the size of PS lenses is directly correlated with that of microdroplets, as shown in Fig. 7.6(a). In the figure, the red areas correspond to that of microdroplets. The yellow and blue areas correspond to that of microlenses fabricated by toluene droplets and toluene films, respectively. The size correlation between PS lenses and PS/toluene droplets is shown in Fig. 7.6.(b). One can see that the radii of lenses linearly increase with that of droplets. The prefactors of the two least-squared fitted lines are 0.78 and 0.71 for the toluene film and toluene droplets generated lenses, respectively. The universal linear dependence between lenses and droplets radius indicates that the concentration of PS in toluene droplets is close to a constant value, regardless the size of droplets. 

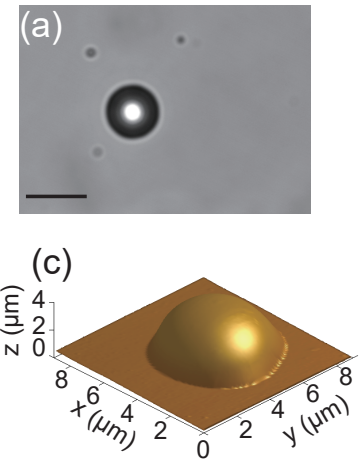
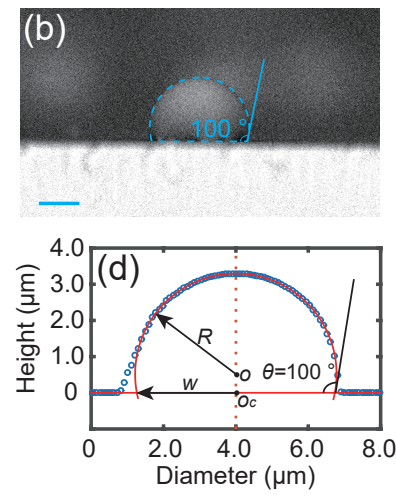

Figure 7.5: Morphological characterization of microlenses: (a) Optical image of a PS lens on a glass substrate (scale bar $5 \mu \mathrm{m}$ ). (b) Side view SEM image of the PS lens, indicating a contact angle of $100^{\circ}$ and spherical-cap-like shape of the lens (scale bar $2 \mu \mathrm{m}$ ). (c) Tapping mode AFM image of the lens. (d) Cross-sectional profile of the lens from the AFM image. By fitting the curve as a circle, a contact angle of $100^{\circ}$ was obtained, which is consistent with the result obtained with the SEM image.

\subsubsection{Mechanism of PS microlens formation.}

To verify the constant PS concentration in toluene/PS droplets, experiments were conducted with different toluene concentrations in toluene/ethanol solution and different thicknesses of PS films, as shown in Figs. 7.6(c) and (d), respectively. The results show that the linear dependence between the size of the microlenses and that of microdroplets is independent of toluene concentration in toluene/ethanol solutions and PS film thicknesses. The prefactors of the linear fitting curves are 0.77 and 0.75 for toluene concentration of $5 \%$ and $8 \%$, and 0.78 and 0.77 for PS film thickness of 38.0 and $77.0 \mathrm{~nm}$, respectively. This implies that the concentration of PS in toluene/PS droplets is a constant value, regardless of the toluene concentration and PS film thickness. Moreover, it implies that the size of microlenses can be tuned by adjusting the size of microdroplets.

According to the above analysis, one can see that the process of microlens formation consists of three key steps, as illustrated in Fig. 7.7. The first step is the precipitation of toluene droplets from toluene/ethanol solution. In the initial binary mixture solution, toluene is uniformly mixed with ethanol, and the PS film remains intact (Fig. 7.7(a)). After water is added, it first 

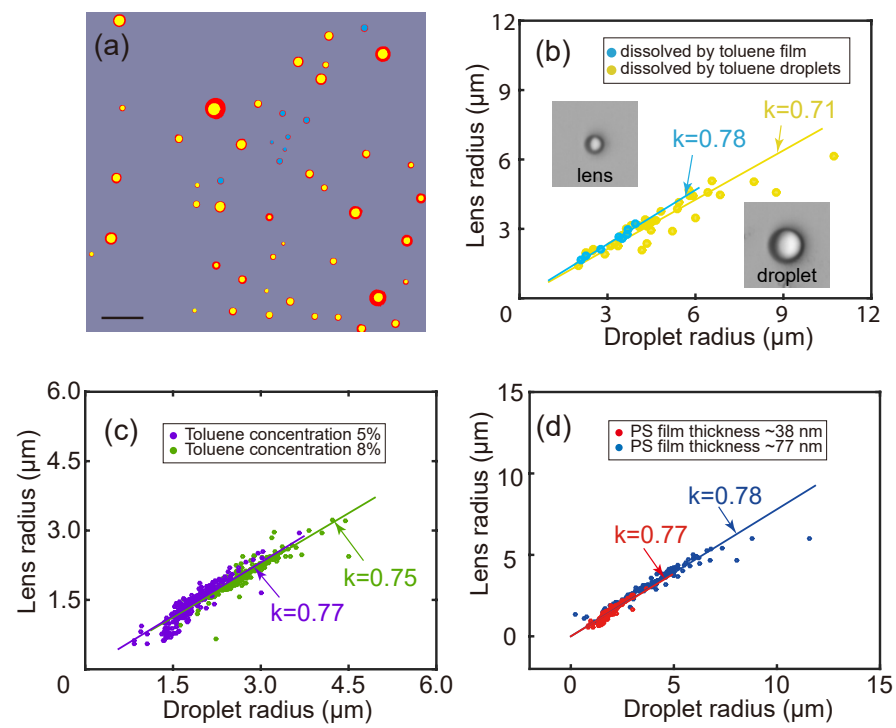

Figure 7.6: Correlation of droplet and lens size. (a) Overlapped binary image of segmented droplets and lenses (scale bar $50 \mu \mathrm{m}$ ). Red areas correspond to initially nucleated microdroplets, and the yellow and blue areas correspond to microlenses generated by direct-deposited microdroplets and toluene film, respectively. (b) Size dependence of microlenses and microdroplets for microlenses generated by the two approaches. It shows a close linear dependence of 0.78 and 0.71. (c) Size dependence of microlenses generated in solutions with different toluene concentration. The obtained linear dependences are 0.75 and 0.78 for toluene concentration of $5 \%$ and $8 \%$, respectively. (d) Size dependence between microlenses and microdroplets for PS films with different thickness. The corresponding values are 0.78 and 0.77 for PS films of 77 and $38 \mathrm{~nm}$, respectively. The universal dependence between the radii of toluene/PS binary droplets and micro PS lenses implies that the PS concentration in toluene/PS droplets remain constant for all binary droplets.

forms a ternary mixture. Since the water is not well mixed initially in the ternary solution, this causes local oversaturation of toluene due to the Ouzo effect [206, 209]. As a result, toluene is precipitated and toluene droplets are nucleated (Fig. 7.7(b)).

In the second step, the precipitated toluene droplets or film attaches on the sample surface and directly dissolves the PS film (Fig. 7.7(c)). This leads to the formation of toluene/PS binary droplets on the substrate (Fig. 7.7(d)). 

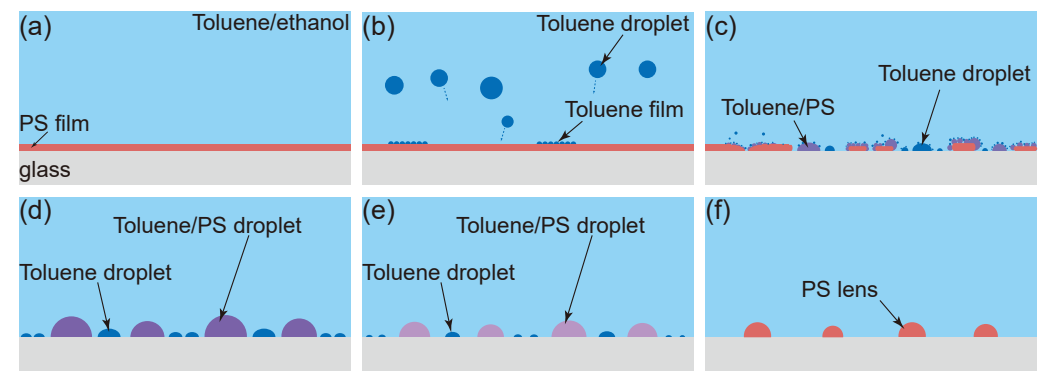

Figure 7.7: Schematic diagram of the process of the microlens formation. The PS film remains intact in the initial toluene/ethanol binary solution (a). The addition of water will cause local oversaturation of toluene and hence the nucleation of toluene droplets (b). The deposited toluene droplets on sample surface will dissolve PS film (c) and result in the formation of PS/toluene droplets on the sample surface (d). After that, the toluene gradually dissolves into the bulk solution with shrinking PS/toluene droplets (e), and eventually PS lenses are left on the sample surface (f).

The third step is the redissolution of toluene into the toluene/ethanol/water ternary solution. After the water is well mixed in the ternary solution, toluene will be redissolved. As a result, the toluene/PS binary droplets gradually shrink with time (Fig. 7.7(e)). Eventually, the PS lenses are formed on the substrate (Fig. 7.7(f)). For some toluene droplets which attach the sample substrate after the PS film is dissolved, they become pure toluene droplets. Eventually they completely disappear.

What is the reason for the constant PS concentration in the toluene/PS droplets, regardless of toluene concentration in the toluene/ethanol binary solution and PS film thickness? We believe that the PS is saturated in the toluene/PS droplets. Right after toluene is deposited on the sample surface, the dissolution of PS film will occur. At the beginning of PS dissolution, there is not enough toluene to dissolve all PS film on the sample surface. As a result, in the toluene/PS droplets, PS remains saturated. With time, more and more toluene deposits on the sample surface and PS are gradually total dissolved. Additionally, it is reported that in the Ouzo effect, the nucleated droplets are covered by a water thin film [20]. The water film acts as a shield and prevents coalescence of the following nucleated toluene droplets. As a result, the PS concentration in the saturated toluene/PS remains saturated.

In the above proposed method, the toluene/PS microdroplets were formed 
by slow adsorption of dissolved toluene into the PS film, and shortly after, the fast nucleation and growth of droplets for oversaturated toluene that leads to dissolution of PS films into the droplets. The slowly adsorbed toluene may lead to the instability of the PS film. The subsequently formed toluene droplets take in PS and form binary toluene-PS droplets. From the experiments, we found that two factors are important for the final formation of microlenses. One is the dissolution of the PS films at solid-liquid interfaces. The other is the high concentration of PS in toluene/PS microdroplets. To verify this, two other experiments were applied. In the first one, ethanol was directly added to the toluene/PS solution. The experimental results show that the dissolved polystyrene precipitated right away and no surface PS microlenses were observed. That is because the addition of ethanol in the toluene/PS solution dramatically decreases the solubility of PS in the solution. No toluene/PS droplets were nucleated at the solid-liquid interfaces.

In the second experiment, a PS-coated glass substrate was first immersed into a toluene/ethanol solution. Then a larger amount of water was added to the mixture solution, and stable toluene/PS droplets were generated on the glass substrate. After that ethanol was added into the mixture solution to remove the toluene in PS/toluene droplets, and PS microlenses can be obtained after toluene was extracted. However, the obtained microlenses had very rugged surfaces, possibly due to the fact that the large amount of water led to excessive toluene droplet nucleation. The PS concentration in the obtained toluene/PS droplets was much lower than that of the saturated value. The lower concentration of PS makes it difficult to generate compact microlenses with smooth surface due to the pinning of the droplets while toluene was redissolved into the solution. The process is similar to the process of a drying polymer solution [210] and evaporation of picoliter droplets with colloidal particles 211].

\subsubsection{Tuning of size and contact angle of PS lenses}

Figure 7.8 demonstrates the different size distribution of lenses produced by changing PS film thicknesses under the same toluene concentration in the toluene/ethanol binary solution. When the PS film thickness increases from 18.0 to $234.0 \mathrm{~nm}$ (Figs. 7.8(a)-(f)), the radius of PS lens linearly increases. The averaged radius of lenses increases from 1.0 to $7.6 \mu \mathrm{m}$, as shown in Fig. 7.8(g). Moreover, the density of microlenses $N$ (unit: $\mathrm{mm}^{-2}$ ) rapidly decreases from about $6 \times 103 \mathrm{~mm}^{-2}$ to about $140 \mathrm{~mm}^{-2}$ with increasing PS film thickness $h$, following the $N \propto h^{-2}$ relationship, as indicated by the solid fitting curve 
in Fig. 7.8 (h).
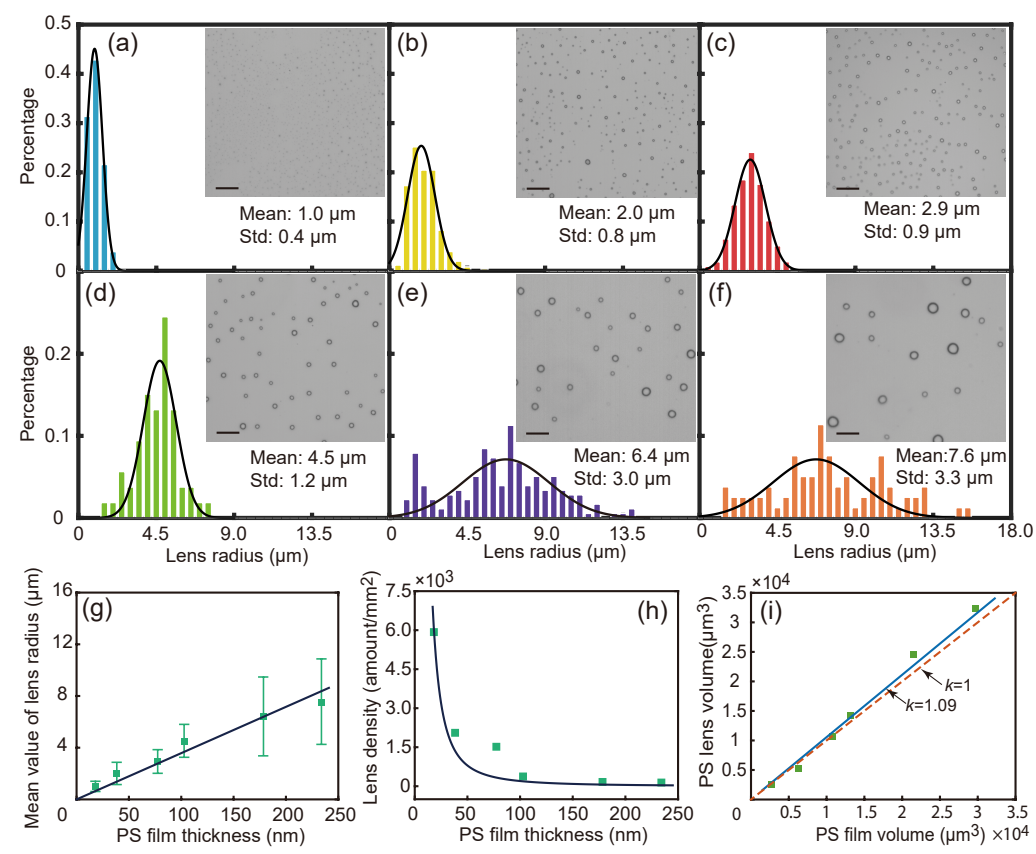

Figure 7.8: Tuning of microlens size and density by changing PS film thicknesses. (a-f) Size distribution of the PS lenses obtained on samples with PS film thickness of 18.0 (a), 38.0 (b), 77.0 (c), 102.0 (d), 179.0 (e), and 234.0 $\mathrm{nm}(\mathrm{f})$. The insets are optical microscope images of the lenses (scale bars: $50 \mu \mathrm{m})$. (g) Correlation between lens radius and PS film thickness. The lens radius increases with PS film thickness. (h) Correlation between lens density and PS film thickness. The lens density $N$ decreases with PS film thickness $h$, following a relationship of $N \propto h^{-2}$. (i) Volume correlation between the PS lenses and PS films. The total volume of PS lenses is exactly the same as that of the PS films within a certain area.

The -2 power law dependence of the microlens density on PS film thickness is due to mass conservation of PS molecules during microlens formation. Since PS lenses are spherical-capshaped, the volume of a microlens can be given as

$$
V_{\text {lens }}=\frac{\pi}{3} R^{3}(2+\cos \theta)(1-\cos \theta)^{2},
$$

where $R$ is the radius of curvature of microlenses and $\theta$ is the contact angle of 
lens. The total volume of all lenses in an area can be approximately given as

$$
\sum V_{\text {lens }} \approx N \times S \times \bar{V}_{\text {lens }}
$$

where $S$ is the area of sample surface and $\bar{V}_{\text {lens }}$ is the average volume of microlenses.

The volume of PS film $V_{\text {film }}$ before microlens formation is given as

$$
V_{\text {film }}=S \times h .
$$

Since the mass is conserved, $\Sigma V_{l e n s}$ and $V_{f i l m}$ are supposed to be equal to each other. By combining Eqs. 7.4-7.6 and $\Sigma V_{\text {lens }}=V_{\text {film }}$, the density of microlenses on the sample surface can be given as

$$
N=\frac{3 h}{\pi R^{3}(2+\cos \theta)(1-\cos \theta)^{2}} .
$$

From the result in Fig. 7.8(g), since the radius $R$ has a linear dependence with PS film thickness, namely, $R \propto h$, the lens density $N$ and PS film thickness $\mathrm{h}$ obey the following relationship:

$$
N \propto h^{-2}
$$

which is consistent with the experimental result shown in Fig. 7.8(h). Since all PS films are converted into lenses, the total volume of PS molecules is supposed to be conserved during the fabrication process, which is experimentally verified. Within the imaging areas shown in Figs. 7.8(a)-(f), we obtained a linear dependence of 1.09 between the volume of PS lenses and that of PS films, as shown in Fig. 7.8(i). This indicates that the total volume of the obtained PS lenses is approximately the same as that of the continuous PS films.

From above analysis, one can see that the size of the generated microlenses increases with the polystyrene film thickness. We relate the increased microdroplet size with increasing PS film thickness to dewetting of PS film induced by the initially adsorbed toluene before toluene is oversaturated and forms droplets. The absorption of toluene by PS leads to the rupture of PS film into domains. The thicker the PS film is, the larger the mass is in a domain. The eventual dissolution of toluene from the droplets results in larger lenses. Meanewhile as revealed by Seemann et al. [212], a PS film with a larger initial thickness increases the characteristic wavelength of the rupturing films. As a result, both toluene/PS droplets size and the distance between adjacent 
droplets increase [198]. As revealed in our experiment, the PS concentration in toluene/PS droplets remains constant (Fig. 7.6). The nucleated toluene/PS droplets on sample surfaces with higher PS thickness can absorb more toluene until PS reaches its saturation concentration level in the toluene/PS droplets. Accordingly, the size of the eventually obtained microlenses increases with PS film thickness.
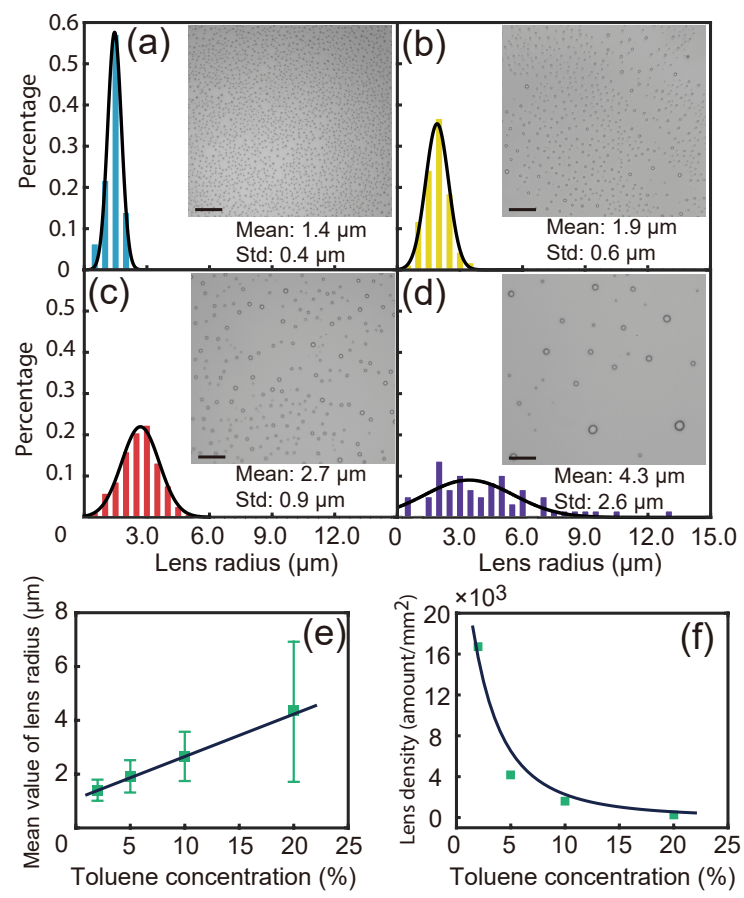

Figure 7.9: Tuning of PS lens size and density by changing toluene concentration with the same PS film thickness of $77.0 \mathrm{~nm}$. (a-d) Size distribution of PS lenses with toluene concentrations of $2 \%$ (a), $5 \%$ (b), $10 \%$ (c), and $20 \%$ (d) (scale bars $5 \mu \mathrm{m}$ ). The radius of PS lenses increases with toluene concentration. The insets are optical microscope images of the PS lenses. (e) Correlation of the mean value of microlens radius with toluene concentration. The size linearly increases with toluene concentration. (f) Correlation of microlens density with toluene concentration. The density decreases with toluene concentration

Besides PS film thickness, toluene concentration can also be used to tune the size and density of microlenses, as shown in Figs. 7.9(a)-(d). For PS films 
with the same thickness of $77.0 \mathrm{~nm}$, the averaged microlens radius increases from 1.4 to $4.3 \mu \mathrm{m}$ when the toluene concentration increased from $2 \%$ to $20 \%$ (Fig. 7.9(e)). Meanwhile, the density of microlenses decreases from about $16 \times 103$ to $240 \mathrm{~mm}^{-2}$. From the results, we can see that the averaged microlens radius roughly has a linear dependence with the toluene concentration. Such dependence is attributed to two coupled effects: the amount of adsorbed toluene by PS film and the size of formed toluene droplets for different toluene concentrations. In the Ouzo effect, the mean size of generated oil droplet is smaller and the size polydispersity is narrower at a lower oil concentration due to the nucleation and growth mechanism established by Katzl et al [20]. In brief, the concentration of toluene limits the number of nucleation sites and the subsequent diffusive growth of toluene droplets. Taking above two facts into account, one can expect that smaller toluene droplets will lead to smaller surface PS/toluene droplets and eventually form lenses with smaller size and reduced polydispersity.
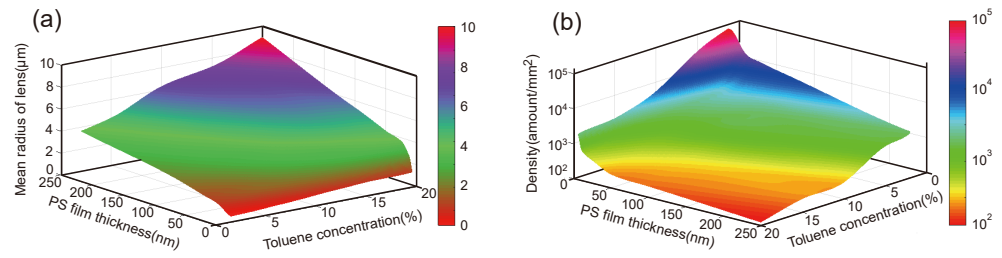

Figure 7.10: (a) Averaged radius of microlenses as a function of PS film thickness and toluene concentration. The averaged radius increases with the PS film thickness and toluene concentration. (b) Lens density as a function of PS film thickness and toluene concentration. The lens density decreases with PS film thickness and toluene concentration.

From above two experiments, one can see that both PS thickness and toluene concentration can be used to tune density and size of microlenses. Here we investigated how the PS lens size and density change when controlling the PS film thickness and toluene concentration at the same time. The investigation is important since it can lead to the controlled fabrication of PS lenses. The averaged radius and density of lenses as the functions of PS film thickness and toluene concentration are presented in Fig. 7.10. Clearly, the averaged radius of the microlenses monotonically increases with increasing toluene concentration and PS film thickness (Fig. 7.10(a)). Regarding density, the results show that it rapidly decreases with increasing PS thickness and toluene concentration, as shown in Fig. 7.10(b). These correlations may 
be used to predict the size and density of the fabricated polymeric microlenses.

The generated microlenses were heated with controlled temperature to tune their contact angle. When polymers are heated above their glass transition temperature, a reversible transition from their hard and glassy state into a viscous or rubbery state will happen 213]. The glass transition is accompanied by the change of contact angle [196]. Here the contact angle of the PS lenses was tuned through keeping the microlenses in a controllable temperature higher than the glass transition temperature.
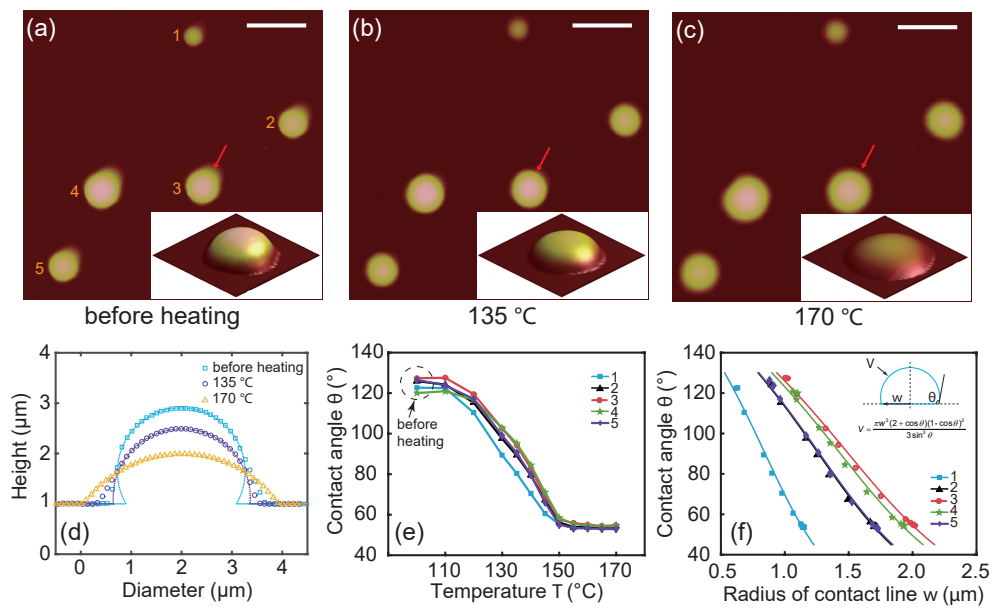

Figure 7.11: Tuning of contact angle through thermal reshaping method. (a-c) Tapping mode AFM image of microlenses on a glass substrate before thermal reshaping, at 135 and $170{ }^{\circ} \mathrm{C}$ for $30 \mathrm{~min}$, respectively (scale bars: $5 \mu \mathrm{m}$ ). (d) Cross-sectional profiles of a microlens marked by arrows in (a-c). The dotted curves are least-squared fitting results by taking the cross sections as spherical caps. (e) The contact angle of microlenses as a function of heating temperature. Contact angle first decreases with the temperature and remains constant after $150{ }^{\circ} \mathrm{C}$. (f) Contact angle as a function of the radius of microlens foot print. The solid curves are obtained by fitting the data points using the inset equation of lens volumes.

Microlenses were characterized by using a tapping mode AFM to measure their contact angles. Figures 7.11(a)-(c) show the raw AFM image of obtained microlenses before and after heating at 135 and $170{ }^{\circ} \mathrm{C}$, respectively. The insets in Figs. 7.11(a)-(c) show the comparison of the 3D images of a lens marked by an arrow. The cross-sectional profiles of the selected microlens 
with fitted circular arcs [183] are shown in Fig. 7.11(d). Clearly, the contact angle decreases with increase in the reshaping temperature.

To tune contact angles of microlenses continuously, 11 different temperatures from 100 to $170{ }^{\circ} \mathrm{C}$ were applied. The contact angle as a function of applied temperature is shown in Fig. 7.11(e). The result shows that the contact angle decreases from about $125^{\circ}$ to about $50^{\circ}$ when the temperature increases from 110 to $150{ }^{\circ} \mathrm{C}$. During the process, we also found that the total volume of PS molecules is conserved. The contact angle as a function of the radius $w$ of the contact line is shown in Fig. 7.11(f). The measured data points were then fitted with the inset equation of lens volume assuming the constant volume is conserved during tuning. For each lens, the measured values of contact angle can be well fitted with the equation. This implies that the $\theta$ and $w$ follows exactly the relationship determined by the equation $V=\frac{\pi}{3}(3 R-H) \times H^{2}$ under constant volume assumption.

\subsubsection{Enhancement of optical imaging with PS microlenses.}

The schematic of the imaging enhancement is shown in Fig. 7.12(a). The microlens sample was placed between an objective lens (NA/0.55) of 50× magnification and a DVD disk in an upright optical microscope (BX51, Olympus, Japan). The imaging resolution can then be enhanced through the fabricated lenses.

The DVD disk was first scanned by the tapping mode AFM. The obtained pitch distance is about $340 \mathrm{~nm}$, as shown in Fig. 7.12(b). After that, the sample was imaged under the optical microscope at wavelength of incident illumination $\lambda=405 \mathrm{~nm}$. Figure 7.12 (c) shows an optical image of the DVD disk without microlenses. In the image, four track pitch gives about $2.9 \mu \mathrm{m}$, which is very close to the value measured by the AFM. Additionally, in the optical image, one can barely see the groove structures of the DVD disk due to the low imaging resolution.

The DVD disk was further imaged with the fabricated microlenses of 15 $\mu \mathrm{m}$ in diameter with the contact angle about $50^{\circ}$ and $30 \mu \mathrm{m}$ in diameter with the contact angle about $115^{\circ}$, as shown in Figs. 7.12(d) and (e), respectively. The insets in the two figures are the enlarged images of areas with the same length scale as Fig. 7.12(c). Both cases provide improved imaging quality with well-recognized grooved structures, compared with the image obtained without microlenses. Moreover, the distance of four track pitches increases to $4.0 \mu \mathrm{m}$ in Fig. 7.12(d) and to $5.2 \mu \mathrm{m}$ in Fig. 7.12(e). This corresponds to an extra magnification of 1.4 and 1.8 for the two microlenses, respectively. 

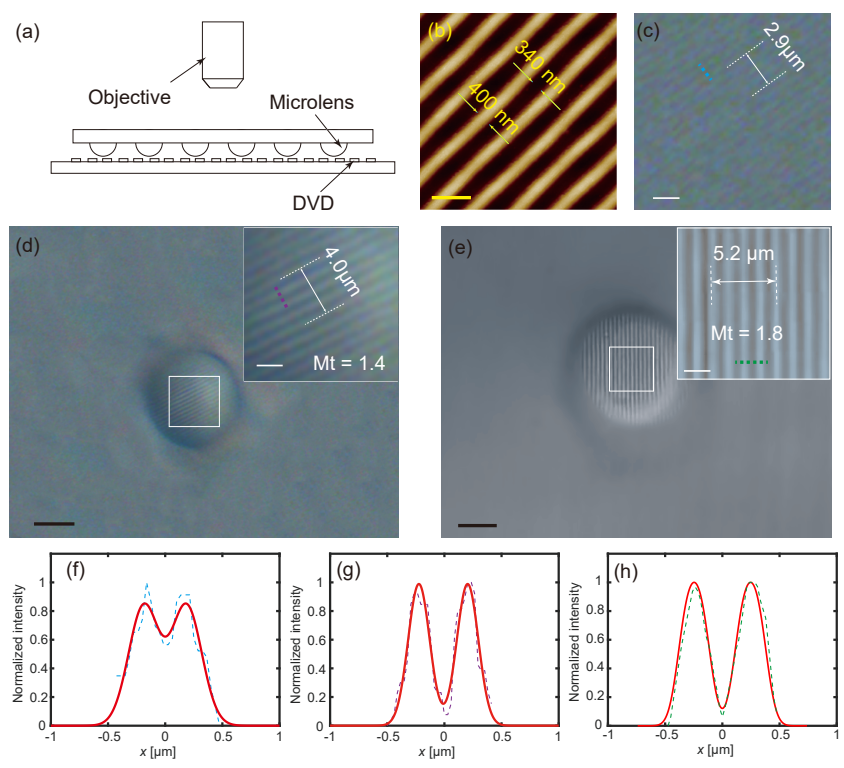

Figure 7.12: Optical imaging enhancement with the fabricated microlenses. (a) Schematic diagram of experimental setup for imaging enhancement using microlenses in an upright optical microscope. (b) A tapping mode AFM image of microgroove structures on a DVD sample surface (scale bar $1 \mu \mathrm{m}$ ). (c) Optical microscope images of the DVD sample surface without microlenses (scale bar $2 \mu \mathrm{m}$ ). (d) and (e) Optical microscope images of the DVD sample using spheres with contact angles of $50^{\circ}$ (d) and $115^{\circ}$ (e) (scale bars: black $10 \mu \mathrm{m}$, white $2 \mu \mathrm{m})$. The insets in the two figures are enlarged images of the selected areas. (f,g,h) Measured (dashed line) and calibrated (solid curve) convolution results of cross-sectional profiles in $(\mathrm{c}, \mathrm{d}, \mathrm{e})$. The groove structures on the DVD sample can barely be observed without microlenses. After microlenses were applied, optical images with enhanced resolution was obtained. Extra magnifications of 1.4 and 1.8 were obtained for microlenses with diameters of 15 and $30 \mu \mathrm{m}$, respectively.

Moreover, the imaging resolutions for images shown in Figs. 7.12(c)-(e) were evaluated. Because the image generated by a microscope is the convolution of a point-spread function (PSF) and the object intensity distribution function, the resolution of a microscope with microlenses can be evaluated through corresponding PSFs of imaging systems [214,215]. As what was done in somewhere else [214], here the rectangular-shaped cross-sectional profiles of 
the DVD tracks were taken as the references to extract corresponding Gaussian PSFs for different imaging systems. According to Houston's criterion [215], the full width at half-maximum (fwhm) of the PSF was taken as the imaging resolution. Figures 7.12 (f)-(h) show three cross sectional profiles obtained from Figs. 7.12 (c)-(e) and the fitting results. Imaging resolutions of $1 / 0.9 \lambda, 1 / 1.7 \lambda$ and $1 / 1.9 \lambda$ are obtained for the microscope without microlenses and with two microlenses with different contact angles of $50^{\circ}$ and $115^{\circ}$, respectively. It clearly shows that the fabricated PS lenses improve imaging resolution. Additionally, microlens with the higher contact angle provides a larger magnification compared with the microlense with the lower contact angle, given the fact that the size of microlenses does not influece magnification of optical imaging [194,207]. This is consistent with a recently reported result that the increasing contact angle favors optical imaging and reaches the optimized performance at around $125^{\circ}$ [193].

\subsection{Conclusions}

In summary, we demonstrated a simple approach to fabricate PS microlenses using transient droplets in a ternary solution. By addition of water into a binary toluene/ethanol solution, toluene droplets spontaneously formed on a solid coated with PS films immersed in the solution. The nucleated toluene droplets dissolve PS film and form PS/toluene mixture droplets. As the toluene in the mixture droplets was extracted into the surrounding ternary solution, PS microlenses were created on the supporting glass substrate. By adjusting PS film thickness and toluene concentration in the toluene/ethanol binary solutions, both size and density of the microlenses can be well controlled. Moreover, contact angle of microlenses was continuously tuned in the range of $50^{\circ}-125^{\circ}$ by curing microlens-decorated surfaces at different temperature. The obtained microlenses can increase the spatial resolution of an optical microscope by 1.4 - and 1.8 -folds with two microlenses with the contact angle of $50^{\circ}$ and $115^{\circ}$. The proposed process is easy to be conducted and exhibits good controllability of surface microlenses, which is of significance for microlensrelated applications. 


\section{Chapter 8}

\section{Sequential evaporation induced formation of polymeric surface microdents via ouzo effect 1}

Microdents fabricated on solid substrates have numerous applications, such as containers for cell growth, reactors for chemical reactions, and nucleation sites for growing photonic crystals. In this study, a new method is developed to fabricate microdents on polystyrene (PS) film deposited on a solid surface. The process relied on Ouzo effect in a ternary solution consisting of three solvents exhibiting different volatilities. In the ternary solution, precursor water droplets are first nucleated when a good solvent of water evaporated. The nucleated water droplets then act as templates for microdent formation during PS film deposition. After water droplets eventually evaporates, microdents are generated on the PS film. The detailed process of microdent formation is experimentally revealed. By controlling the nucleation of water droplets and PS concentration, the lateral size and the depth of microdents are systematically tuned. Moreover, it is demonstrated that the microdent decorated films obtained with the proposed method can be used to fabricate lubricating surfaces, which exhibit a low contact angle hysteresis of $2^{\circ}$.

\footnotetext{
${ }^{1}$ Published as: Yuliang Wang, ${ }^{*}$ Binglin Zeng, Xiaolai Li, and Xuehua Zhang, ${ }^{*}$ Sequential evaporation induced formation of polymeric surface microdents via ouzo effect, Adv. Mater. Interfaces 6, 1900002 (2019). Experimental work is done by Zeng.
} 


\subsection{Introduction}

Microdents on solid surfaces have been applied in numerous applications. They can serve as containers for cell growth, [216, 217] microreactors for chemical reactions [218], platforms for droplet manipulation [185], nucleation sites for photonic crystals [219, 220] and micro/nanobubbles [179, 180, 184, 221]. Moreover, surface microdents can potentially act as scaffolds for surface lubricants in the fabrication of lubricating surfaces [222], which exhibit low contact angle hysteresis and can be used in many applications, such as selfcleaning [223], anti-icing [224] and enhanced condensation [225].

Several approaches have been developed to fabricate microdents on solid polymer surfaces, such as soft lithography method [226], nonsolvent sessile droplets action [227], solvent droplet etching method, [228 230] and templating methods [217, 231 235]. In the soft lithography method [226], microlenses and a positive photoresist substrate are used to get microdent arrays. In the solvent droplet etching method, a single [228, 230] or a group of solvent droplets [229] on top of micropillars are transferred to polymer films. The corresponding spots on the polymer films will be etched and microdents can then be generated. In order to obtain smaller microdents, smaller size of solvent droplets can be generated through solvent exchange method [171, 206]. In the templating methods, colloidal particles [217, 231, 235, 236] or water droplets [232 234] (breath figure method) are taken as templates. The templates are first placed on a thin film of solvent with dissolved polymer. After the solvent evaporates, microdents are generated on the spots where the templates locate. Compared with the lithography and solvent droplet etching methods, templating methods are easy to be conducted and are more suitable to fabricate microdents in batches.

Recently, "Ouzo effect" was applied to fabricate micro/nanostructures, such as micro/nanodroplets [237], micro/nanoparticles [238], and micro/nanolenses [170,206]. "Ouzo effect" is referred to the nucleation of tiny oil droplets after water is added into a Greek drink of Ouzo [20, 156, 239]. Since the solubility of oil in ethanol-water solution decreases with increase in the water concentration, the oil is oversaturated, spontaneously forming microdroplets after more water is added in the oil-ethanol-water ternary solutions. Instead of adding water into ethanol-oil mixture, evaporation of ethanol in an Ouzo drop can also trigger Ouzo effect [19]. The Ouzo effect provides an efficient approach to produce microdroplets that in turn serve as templates for the formation of micro/nanostructures [170,237, 238]. Compared with the existed methods, mi- 
cro/nanostructure fabrication via Ouzo effect can be conducted without any special setups and complex operations.

Here we propose a new templating method to fabricate surface microdents via "Ouzo effect" in a ternary solution. By designing the solution of polystyrene (PS) in the ternary solvents with different volatilities, water droplets can form at solid-liquid interfaces, followed by the formation of a PS film. The nucleated water droplets act as templates for the fabrication of microdents on the PS film. In this study, the mechanism of microdent fabrication will be revealed experimentally, together with the demonstration of the control of the lateral size and depth of microdents. Moreover, we will demonstrate that the obtained microdents can be used to fabricate lubricating surfaces, on which the surface lubricant-silicone oil-impregnates the microdents and is stabilized by capillary force arising from the microdents.

\subsection{Experimental section}

\subsubsection{Sample preparation}

The prewashed coverslips (Menzel Glaser, VWR, UK) of $2 \mathrm{~cm} \times 2 \mathrm{~cm}$ were taken as substrates for surface microdent fabrication. Before experiments, they were first cleaned with nitrogen gas flow. Strict cleaning procedure was unnecessary because that acetone and toluene both had very low interfacial energy and low contact angles on glass substrates in air. The low interfacial tension of the solvents ensured that the process does not require strict cleaning procedure to obtain low contact angles of the liquid on the underlying substrate. A contact angle meter (OCA 15Pro, Dataphysics, Germany) was employed to measure the wettability of the coverslips. Figures 8.1(a)-(c) are the optical images used for the measurement of static contact angles of water, toluene, and acetone on the coverslips, respectively. The values were $28.2^{\circ}$, $7.2^{\circ}$, and $5.3^{\circ}$ for water, toluene, and acetone, respectively.

\subsubsection{Preparation of the ternary solution and fabrication of microdents}

The ternary solution of toluene-acetone-water with PS was prepared by mixing a PS/toluene solution and a water/acetone solution. PS particles (molecular weight 350000, Sigma-Aldrich) without any active groups were dissolved into toluene (analytical reagent, Aladdin, China) at different concentrations of $1.2 \%, 1.6 \%$, and $2.0 \%$ (weight) for the preparation of PS/toluene solution. For 
(a)

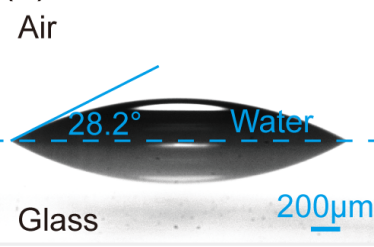

(b)

Air (c)

Air

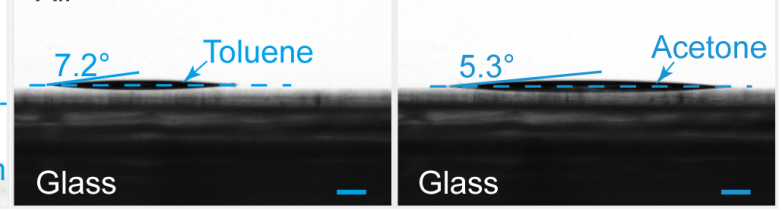

Figure 8.1: Static contact angles of (a) water, (b) toluene, and (c) acetone on the coverslips.

water-acetone solution, deionized (DI) water and acetone (analytical reagent, Aladdin, China) were mixed with water concentration of $8 \%, 10 \%, 12 \%$, and $14 \%$ (weight).

The two prepared solutions were then mixed with a ratio of 3:1 (toluene-PS to acetone-water). The mixing of the two solutions caused reduced solubility of PS. As a result, part of PS precipitated and the toluene/acetone/water ternary solution with PS was obtained. The ternary phase diagram of the acetone, toluene, and water was redrawn from Walton and Jenkins (1923) [240] and is shown in Fig. 8.2. For fabrication of microdents, ternary liquid of $200 \mu \mathrm{L}$ was dropped onto the prepared glass substrates in a petri dish. After microdents were fabricated, the microdent-decorated samples were put into an oven (DZF, LICHEN, China) at $40^{\circ} \mathrm{C}$ for $4 \mathrm{~h}$ to remove the remaining solvent.

\subsubsection{Morphological characterization of microdents}

An inverted optical microscope (IX73, Olympus, Japan), an AFM (Resolve, Bruker, USA), and an infrared imaging microscope (iN10MX, Nicolet, America) were used to characterize microdents. The optical microscope was used to capture the dynamic process of microdent formation, associated with a high-speed camera (EoSens 3CL, Mikrotron, Germany). A home designed program was applied to achieve the automated segmentation of the obtained images [66, 67, 183]. From the program, the size of microdents can be automatically extracted. The tapping mode AFM was used to characterize the microdents in a 3D manner. From AFM images, the depth of microdents can be obtained. The infrared imaging microscope was employed to detect the components at microdent bottom. 


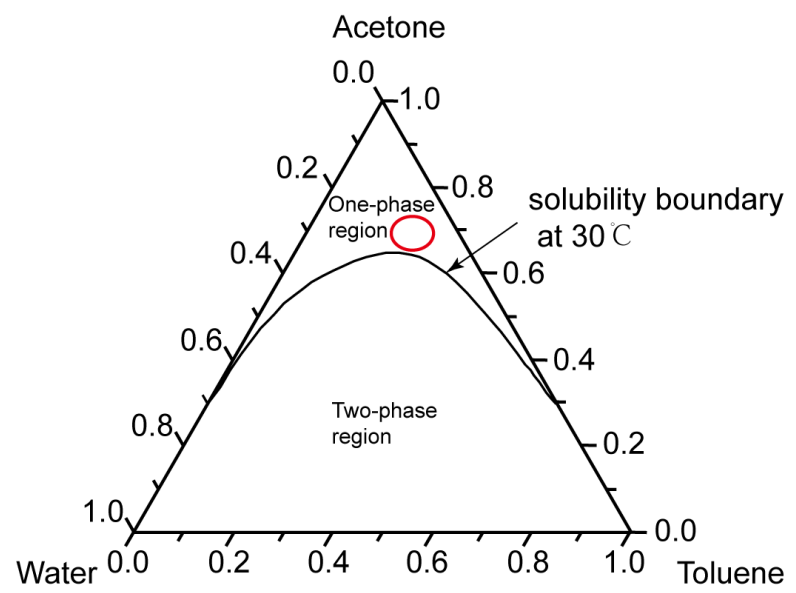

Figure 8.2: Partial phase diagram of acetone, toluene, and water, and the mutual miscibility curves at $30^{\circ} \mathrm{C}$, redrawn from Walton and Jenkins (1923). [240] The red circle indicates the region where the concentrations of different solvents were selected.

\subsubsection{Contact angle hysteresis of water droplets on the lubri- cating surface}

To evaluate the mobility of water droplets on the fabricated lubricating surface, a $5 \mu \mathrm{L}$ water droplet was dropped onto the surface. The tilting angle of the sample surface gradually increased until the water droplet slipped downwards along the surface. The dynamic contact angles were measured with the contact angle meter. For comparison, one also tried to measure the dynamic contact angles of the water droplets on a smooth PS film and a microdent-decorated PS film without silicon oil film. However, water droplets were pinned on both surfaces even when the tilting angle reached $90^{\circ}$.

\subsection{Results and discussion}

\subsubsection{Fabrication of microdents}

Figure 8.3 shows the experimental procedure for the microdent fabrication on the glass substrate and schematic diagrams of the experimental process. The obtained optical images of the sample surface are shown in Figs. 8.3(f)(j). A glass substrate was put in a petri dish and the ternary solution of 
toluene/water/acetone with precipitated PS particles was deposited into the petri dish (Figs. 8.3(a),(f)). Then the turbid liquid was spread out on the glass surface (Figs. 8.3(b),(g)). In the ternary solution, acetone has the highest evaporation rate, followed by toluene and water (see Table 8.1 in the Appendix) [241]. As a result, acetone evaporated first in the deposited ternary solution, Ouzo effect took place, and surface water droplets started to nucleate. The increase of the toluene concentration in the ternary solution leads to dissolution of PS particles again (Figs. 8.3(c),(h)). After acetone evaporated, toluene started to evaporate. This led to the precipitation of PS in the form of a thin film on the glass substrate. Since water droplets nucleated on the substrate, the PS film mainly covered the areas without water droplets. After toluene entirely evaporated, water droplets were exposed to the air (Figs. 8.3(d),(i)). Because water droplet nucleation occurred before PS film generation, eventually microdents were formed after water droplets completely evaporated (Figs. 8.3(e),(j)). For the detailed process of the design of the ternary solution, readers are referred to Section 8.5.1 in the Appendix.

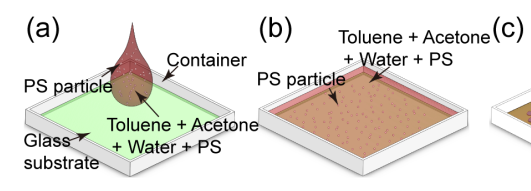

(c)

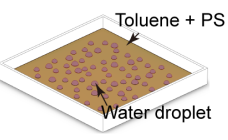

(d)

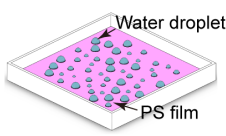

(e)

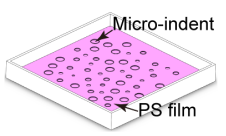

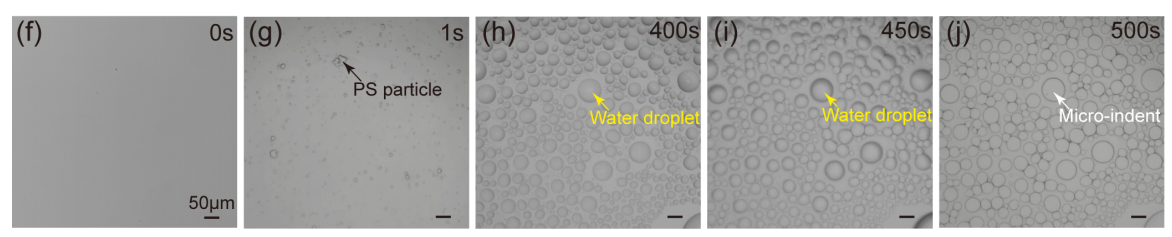

Figure 8.3: (a-e) Schematic diagram and (f-j) corresponding optical images of the experimental procedure designed for microdent fabrication by taking water droplets as templates. (a, f) A drop of mixed toluene/PS and acetone/water solutions was deposited onto a glass substrate. (b, g) PS particles were precipitated due to the reduced solubility in the ternary solution. (c, h) Acetone rapidly volatilized, leading to the nucleation of water droplets at the solid substrate. (d, i) After toluene was completely volatilized, a PS film was formed and water droplets were exposed to the air. (e, j) Eventually water was evaporated and microdents were formed at the spots where water droplets located.

In the process of microdent formation, understanding the temporal evolu- 
tion of water droplets is key to reveal the mechanism of microdent formation. Figure 8.4 depicts the morphological evolution of the nucleated water droplets. Basically two factors contributed to the growth of water droplets. One was the separation of water from the ternary solution due to Ouzo effect. For an existing droplet, the continuous separation of water from the solution led to an increase in the size of the water droplet. For example, the size of droplets $\mathrm{I}^{\prime}$ and $\mathrm{II}^{\prime}$ in Fig. 8.4(b) are slightly increased compared to the droplets I and II in Fig. 8.4(a). This is due to the separation of water from the ternary solution. The other factor was the coalescence of neighboring water droplets. For example, droplets I' and II' and several other droplets nearby in Fig. 8.4(b) coalesced to form droplet III in Fig. 8.4(c).
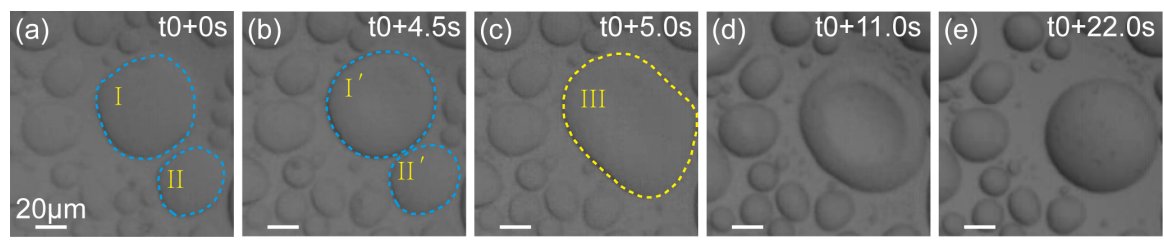

Figure 8.4: Evolution of water droplets in liquid film. (a) Water droplets were generated on the surface, (b) growing with time, (c) coalesced with neighboring ones, (d) partially exposed to air, and (e) eventually exposed to air.

With the evaporation of solution, the water droplets were partially exposed to air (Fig. 8.4(d)). Eventually, all toluene was volatilized and all water droplets were directly exposed to air (Fig. 8.4(e)). Compared to droplets shown in Figs. 8.4(a)-(d), the droplets in Fig. 8.4(e) became more spherical. We speculate that this is due to the change of surface wettability, from hydrophilic to hydrophobic. It is highly possible that there is a thin PS film at the bottom surface of microdents.

Water droplets started to evaporate after exposed to air. The sequential images of a water droplet and the shrinking process in a microdent are presented in Fig. 8.5(a). Initially, the three phase contact line was pinned at the side wall of the microdent, as illustrated in Fig. 8.5(b) (the first). With the evaporation of water, the droplet gradually shrank with decreased height (Fig. 8.5(b) [the second]). When the surface of the water droplet touched the bottom of the microdent (Fig. 8.5(b) [the third]), water droplet became a water ring and was still pinned at the side wall of the microdent (Fig. 8.5(b) [the fourth]). Subsequently, the water ring further shrank (Fig. 8.5(b) [the fifth]) and eventually disappeared (Fig. 8.5(b) [the sixth]). This result shows 
(a)
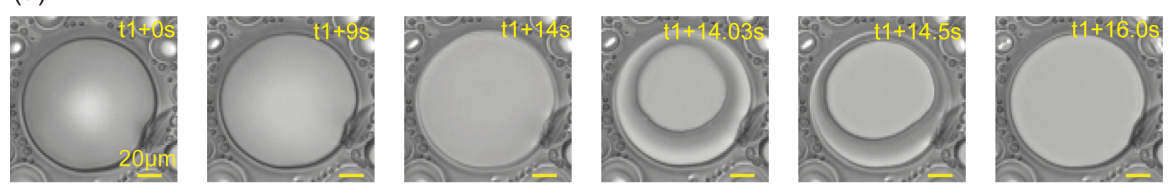

(b)

Initially water droplet
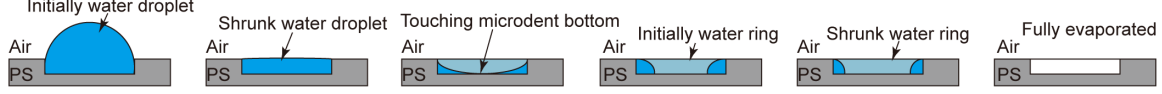

Figure 8.5: Evaporation of a water droplet inside a microdent after fully exposed to air. (a) The sequential optical microscope images and (b) corresponding schematic diagrams of the water droplet in the microdent. The microdent was first completely filled by the water droplet (first column) and gradually shrank with time because of evaporation (second column) until the droplet touched the bottom of the microdent (third column). After that, the water droplet became a ring (fourth and fifth column) and eventually disappeared (sixth column).

a pinning of the droplet to the microdents, which accords with the constant contact radius dissolution mode revealed in our previous work [185].

How PS evolves is also important for understanding the mechanism that governs the microdent formation. The initially deposited liquid contained PS precipitates because of the low solubility of PS in the ternary solution. With evaporation of acetone, water droplets form and separate out from the solution, leading to an increase in the toluene concentration in the solution. As a result, the initial PS precipitates redissolved into the solution. Figure 8.6 shows the dissolution process of the initially precipitated PS particles. In Fig. 8.6. (a), a PS particle is marked by a black arrow. As acetone evaporated, toluene concentration increased and the PS particle redissolved, shown by the reduced size (Fig. 8.6(b)). Eventually, the PS particle disappeared and dissolved completely (Fig. 8.6(c)).

\subsubsection{Characterization of microdents}

An atomic force microscopy (AFM) was used to characterize the obtained microdents. A 2D and corresponding 3D tapping mode AFM height images of microdents are shown in Figs. 8.7(a) and (b), respectively. The AFM images reveal that the bigger microdents have flat bottoms while the smaller ones have round bottoms. This can also be seen from the enlarged 3D images and 


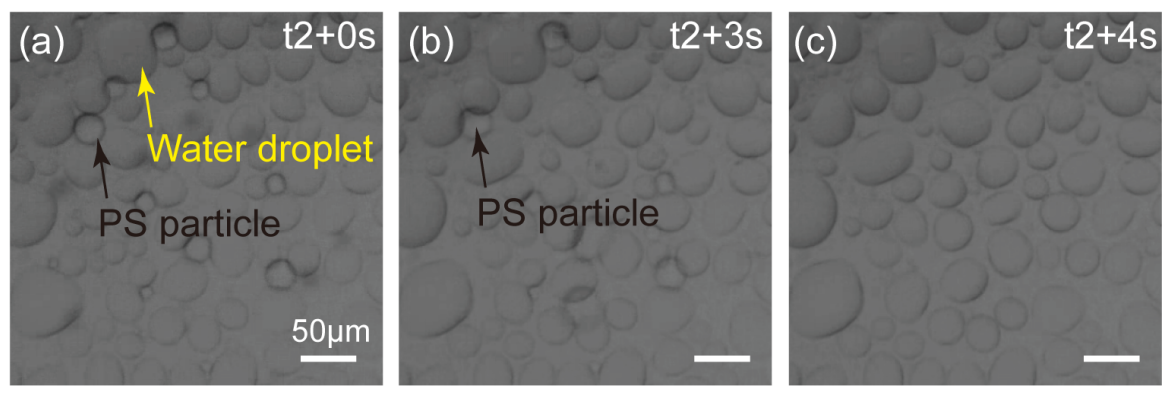

Figure 8.6: Dissolution of PS particles into solution during the process of water droplet nucleation. (a) PS particles were first precipitated in the ternary solution. (b) PS particles started to dissolve into the solution. (c) PS particles were totally dissolved into the liquid.

corresponding cross-section profiles of the two selected microdents, as shown in Figs. 8.7(c) and (d). Correlation between the depth and the diameter for the two different types of microdents is given in Fig. 8.7(e). For the flat bottom microdents, the depth slightly increases with the diameter. Theoretically, an area with larger microdents has reduced coverage for the deposited PS, leading to an increased thickness of the deposited PS film. Additionally, the microdents with round bottoms have much lower depth than that of flat bottom ones.

A thin film of PS at microdent bottom is verified by two tests as following. The first one is AFM lithography experiment. The bottom surface of a microdent was scratched with an AFM tip. Figure 8.8(a) shows the AFM height image of a microdent before the scratching. The corresponding AFM height image for the area with the scratch and its cross-section profile is shown in Fig. 8.8(b). The result clearly shows that there was indeed a thin film at the microdent bottom. Furthermore, the material at microdent bottom can be chemically identified to be PS from infrared absorption spectrum measurements. Figure 8.8(c) is an optical image of the sample used for infrared absorption spectrum measurement. In the area, two points were selected. One was at the bottom of a microdent (marked by a blue cross), and the other was in an area outside microdents (marked by a red cross). The measurement results are shown in Fig. 8.8(d). From the result, one can see that both curves exhibit similar detected peaks. The methylene and benzene ring in PS were detected according to the corresponding absorption peaks, such as 2926, 2853 $\mathrm{cm}^{-1}$ for methylene, and 3000-3100 $\mathrm{cm}^{-1}, 1450-1600 \mathrm{~cm}^{-1}, 770-730 \mathrm{~cm}^{-1}$ for 

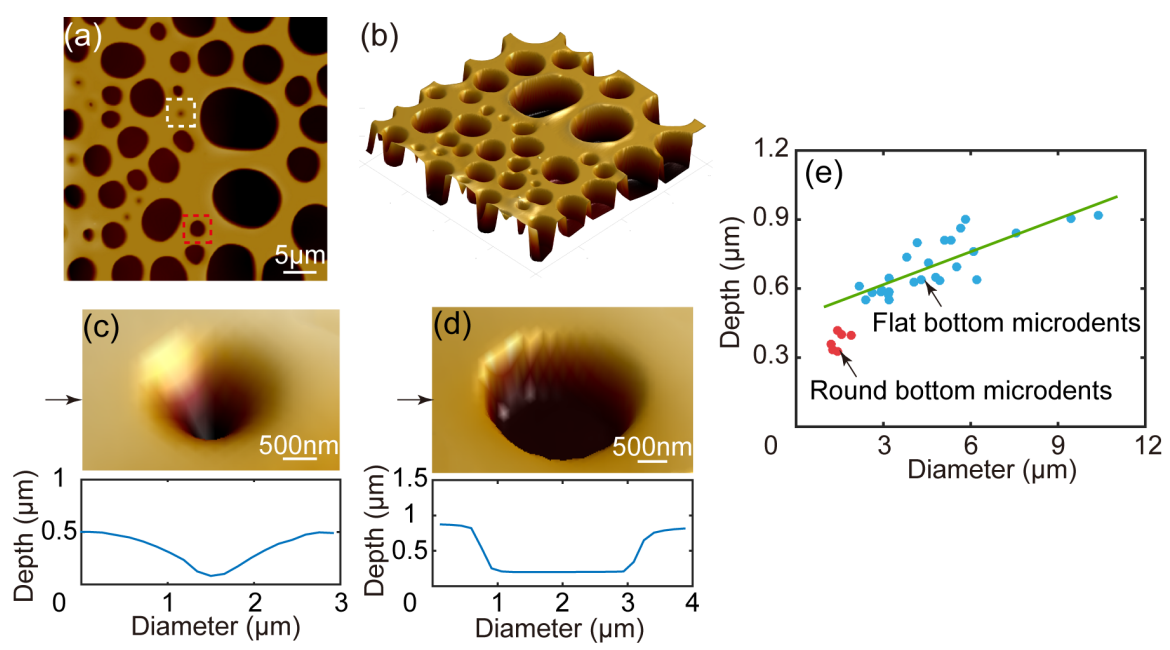

Figure 8.7: Morphological characterization of microdents using an AFM. (a,b) The 2D and 3D tapping mode AFM images of microdents on a PS film. (c,d) 3D AFM images and corresponding cross-section profiles of a small microdent with a round bottom and a larger microdent with a flat bottom, respectively. (e) Depth as a function of the diameter of microdents. Blue and red dots correspond to microdents with flat and round bottoms, respectively.

benzene ring. The result clearly indicates that the bottom of the microdent was covered by a PS film.

The mechanism of the thin PS film formed at microdent bottoms is revealed. In the beginning, water droplets gradually appear in the toluene/PS environment with the evaporation of acetone. Since toluene has a smaller surface energy than water [241], it can easily spread out on a glass substrate. Therefore, water droplets do not directly contact the glass substrate [242]. There is a thin film of toluene-PS solution in between water droplets and the glass substrate. Eventually, the evaporation of toluene results in oversaturation and hence the precipitation of PS in the form of thin film at the glass substrate. The formation of hydrophobic PS film increases contact angle of surface water droplets and the water droplets are reshaped into spherical cap-liked shape [243].

What determines the lateral size of microdents? Experimental results show that the lateral size of microdents is directly correlated with that of water droplets, as shown in Fig. 8.9(a). In the figure, the white areas correspond to that of water droplets. The blue contours correspond to the boundaries 

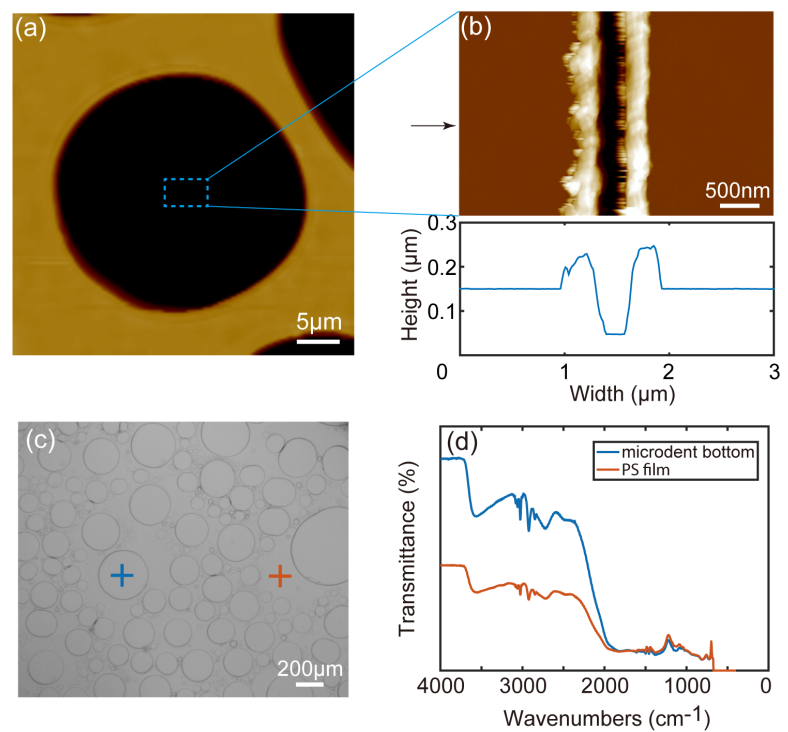

Figure 8.8: (a) An AFM height image of a microdent before scratching. For the selected area, higher load was applied to scratch the bottom surface of the microdent. (b) The AFM height image of the selected area after scratching with an AFM cantilever. It is clear that the bottom surface was covered by a thin film of about $100 \mathrm{~nm}$ in thickness. (c) An optical image of the sample used for infrared absorption spectrum measurement. The measurement locations are marked by a blue and a red cross for a microdent bottom and the PS film outside microdents. (d) Infrared absorption spectrum measured at the microdent bottom and outside the microdents. Both measurements indicate that the microdent bottoms are actually covered by a thin PS film.

of microdents. The size correlation between microdents and water droplets is shown in Fig. 8.9(b). One can see that the radii of indents linearly increase with that of water droplets. The prefactor of the least-squared fitted line is 1.07, which implies that the lateral size of microdents are almost identical to that of water droplets.

\subsubsection{Tuning of microdent lateral size by water concentration}

Based on the principle of microdent formation, one can see that the lateral size of microdents is directly correlated to the footprint diameter of the water droplets, while the depth of microdents are related to the thickness of PS 

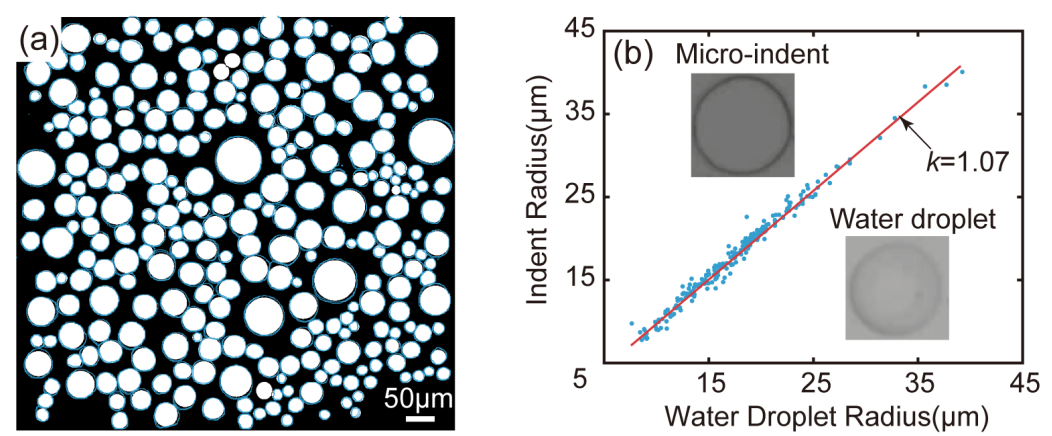

Figure 8.9: Size correlation of water droplets and microdents. (a) The binary image of segmented water droplets and microdents. White areas and blue contours correspond to the nucleated water droplets and microdents, respectively. (b) Microdent radius as a function of water droplet radius, indicating the identical size of the microdents and water droplets.

films. This provides an approach to tune the size of the microdents. Since the ternary solution of toluene/water/acetone was prepared by mixing wateracetone solution and PS-toluene solution in proportion, the nucleation of water droplets can be controlled by adjusting water concentration in water-acetone solution [156,170] and the PS film thickness can be tuned by PS concentration in PS-toluene solution.

Figures 8.10 (a)-(d) shows the optical images of microdents fabricated with water concentrations of $8,10,12$, and $14 \mathrm{wt} \%$, respectively, with the same PS concentration of $1 \mathrm{wt} \%$. Here an area-orientated statistical analysis of the microdents was implemented to better characterize the coverage of microdents on the glass substrates at different water concentrations. The area proportion $\mathrm{S}$ of a microdent is defined as:

$$
P=\frac{A}{\sum_{i=1}^{n} A_{i}} \times 100 \%
$$

where $A$ is the bottom area of a microdent and $\sum_{i=1}^{n} A_{i}$ is the sum of the areas for all microdents in the area. Assuming there are $k$ microdents around a certain lateral size on the sample surface, the sum of the area proportions $S$ for these microdents can be given as:

$$
S=\sum_{i=0}^{k} P_{i} \times 100 \% .
$$



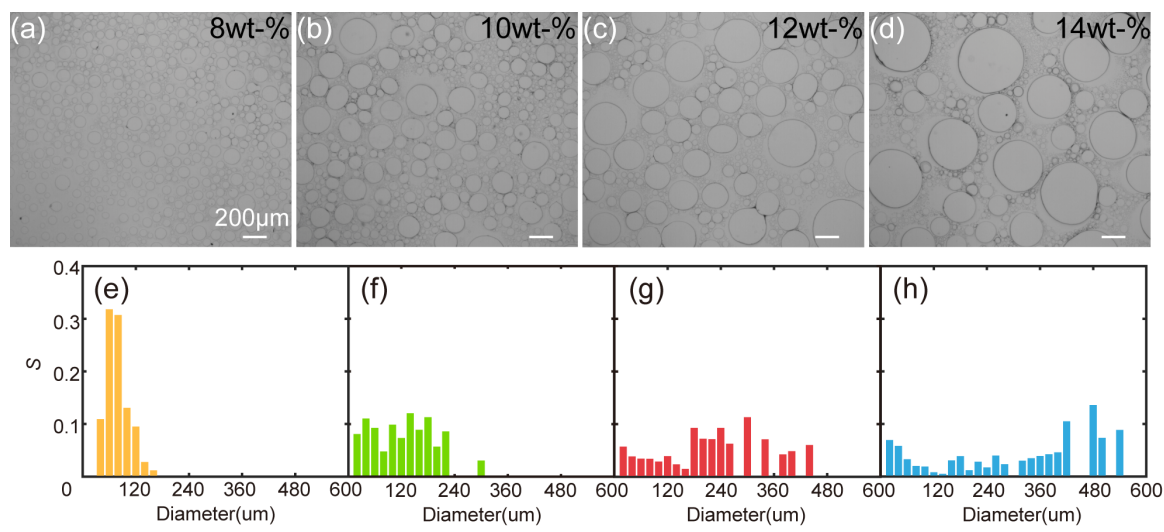

Figure 8.10: Tuning of microdent size by changing water concentration. (ad) Optical images of the microdents. (e-h) Distribution of the sum of area proportion $S$ (Equation (2)) of the microdents at different lateral sizes. The water concentration was (a,e) $8 \mathrm{wt} \%,(\mathrm{~b}, \mathrm{f}) 10 \mathrm{wt} \%,(\mathrm{c}, \mathrm{g}) 12 \mathrm{wt} \%$, and $(\mathrm{d}, \mathrm{h})$ $14 \mathrm{wt} \%$.

For the obtained microdents, the sum of the area proportions $S$ as a function of microdent lateral sizes are shown in Figs. 8.10(e)-(h). When the water concentration increases from 8 to $14 \mathrm{wt} \%$, the range of microdent size increased from $0-200 \mu \mathrm{m}$ to $0-600 \mu \mathrm{m}$.
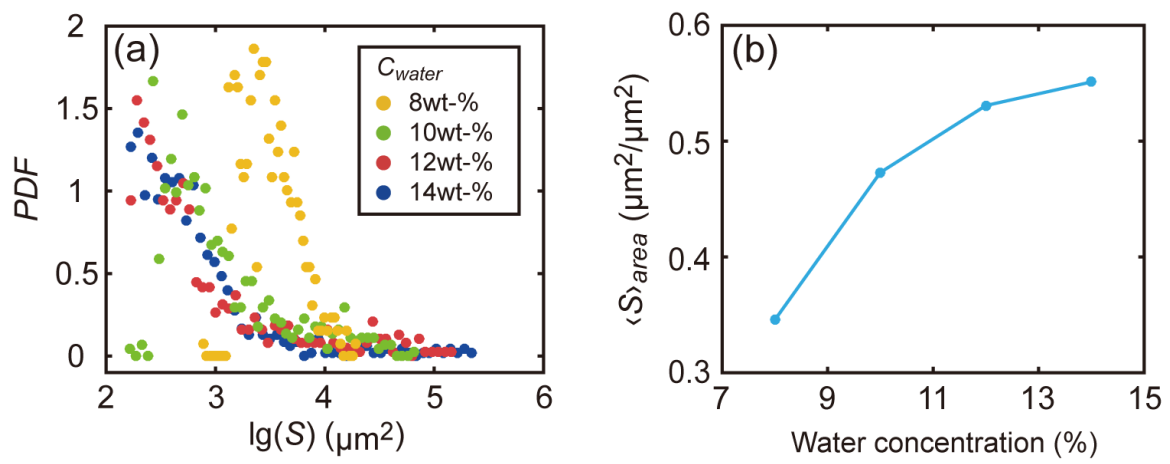

Figure 8.11: Statistical analysis of size distribution of the produced microdents. (a) PDF of microdent area at different water concentrations. (b) The averaged area per unit area $\langle S\rangle_{\text {area }}$ for the produced microdents with different water concentration values. 
Figure 8.10 shows that the size of microdents at water concentration of $8 \%$ is obviously smaller than those at $10-14 \%$. The difference of size distribution of microdents in between the lower and higher water concentrations can also be seen from the probability density function (PDF) analysis, as shown in Fig. 8.11(a). The PDF of microdent area indicates the probability of microdent areas at specifc values. The result shows that the distribution of the microdent area became wider at three higher water concentrations than the situation of $8 \%$. Moreover, three cases with higher water concentrations exhibit similar PDF curves, in contrast to the situation of $8 \%$. The average value of microdent area per unit area $\langle S\rangle_{\text {area }}$ at different water concentration is plotted in Fig. 8.11(b), which shows a rapid increase of the indent size with increasing water concentration.

The dependence of the microdent size on water concentration is attributed to the correlation between the size of the water droplets from the Ouzo effect and the initial water concentration. Our recent work has shown that the initial solvent ratio determines the oversaturation and hence the size of the formed droplets under constant flow conditions by solvent exchange [181], an analogy to the evaporation-driven Ouzo effect. It was found that the droplet size does not follow a simple linear relationship with the initial solvent ratio, but complicated by the exact solubility phase diagram for specific ternary combination. This may contribute to the much smaller droplets formed at $8 \%$ water. Furthermore, it is known that there is a plateau in the surface coverage of the droplets due to droplet coalescence [179], same to our situation here as the water droplets were produced on the surface. The much smaller droplets in $8 \%$ water may also be due to the reduced probability in droplet coalescence as the initial surface coverage of the droplets is smaller.

\subsubsection{Tuning of microdent depth by PS concentration}

Besides the lateral size, the depth of microdents can also be tuned by adjusting PS concentration in toluene-PS solutions. Figures 8.12(a)-(c) shows the AFM height images of microdents fabricated with different PS concentrations of $1.2 \%, 1.6 \%$, and $2.0 \%$, respectively, with the water concentration in wateracetone solution at $10 \%$. Since the depth of microdents is size dependent, three microdents with similar size are selected from Figs. 8.12(a)-(c) to compare the microdent depth obtained at different PS concentrations. Their cross-section profiles are given in Fig. 8.12(d). From the figure, one can see that the depth of microdent increases with increasing PS concentration. Figure 8.12(e) shows the correlation of the depth with the diameter of microdents from Figs. 
8.12 (a)-(c). It is obvious that higher PS concentration led to larger depth of microdents. Therefore, different from the traditional breath figure method, through which the microdent depth is usually difficult to control, microdent depth can be tuned well by this proposed method.
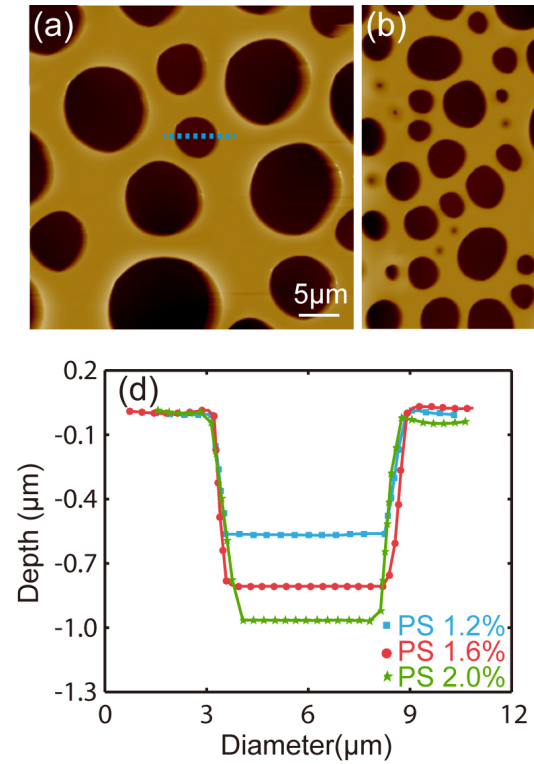
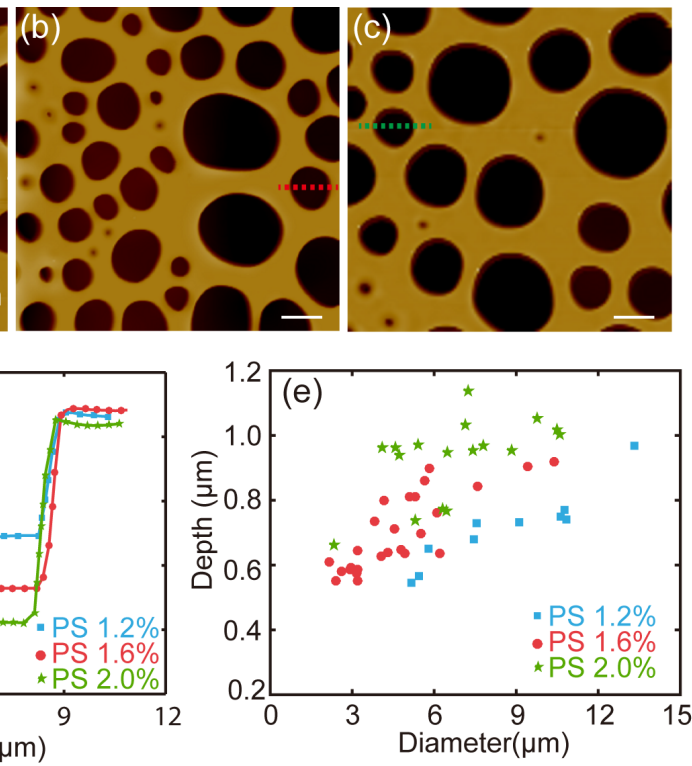

Figure 8.12: Tuning of microdent depth by adjusting PS concentration. (a-c) Tapping mode AFM images of microdents with PS concentration of 1.2, 1.6, and $2.0 \mathrm{wt} \%$, respectively. (d) Comparison of cross-section profiles of microdents with similar size and marked by three dashed lines in (a-c). (e) Depth versus lateral size of microdents obtained with different PS concentrations. The depth of microdents increase with increasing PS concentration.

\subsubsection{Lubricating surface fabrication with microdent decorated PS films}

The as-prepared surfaces decorated with microdents may find many potential applications. Here we demonstrate the application of microdent-decorated PS films in fabricating lubricant-infused surfaces by simply coating a surface lubricant of silicone oil on the obtained PS surfaces. Silicone oil impregnates microdents and is stabilized by capillary force arising from microdents to obtain lubricating surfaces, as illustrated by Fig. 8.13(a). For comparison, 5 
$\mu \mathrm{L}$ water droplets were first dropped onto a smooth PS film and a microdentdecorated PS film. For both cases, when the tilt angle was 90 were still pinned on the two surfaces, as shown in Figs. 8.13(b) and (c). On the fabricated lubricating surface, the spreading coefficient $S_{o w(a)}$ of silicon oil on water surface is $6 \mathrm{mN} \mathrm{m}^{-1}$, which is larger than zero. As a result, silicon oil cloaks over the water droplet [222]. Figure 8.13(d) shows the optical image of a sliding water droplet on the tilted lubricating surface with a tilt angle of $11^{\circ}$. The obtained advancing and receding contact angles are $76^{\circ}$ and $74^{\circ}$, respectively, which gives a low contact angle hysteresis of $2^{\circ}$. The results show that the lubricating surfaces fabricated with the microdent-decorated films can significantly increase the mobility of water droplets.
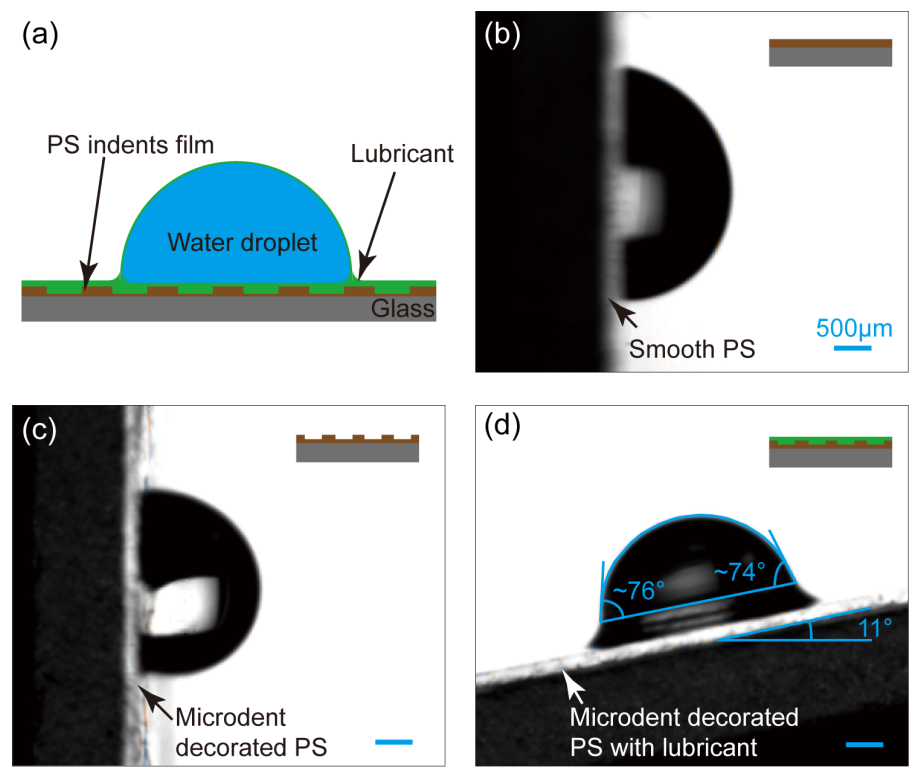

Figure 8.13: (a) Schematic diagram of a water droplet placed on a microdent surface impregnated with silicone oil. (b,c) Optical images of water droplets pinned on a (b) smooth PS film and a (c) microdent-decorated PS film. (d) An optical image of a sliding water droplet on a lubricating surface obtained by impregnating silicone oil on a microdent-decorated surface, exhibiting a $2^{\circ}$ contact angle hysteresis. 


\subsection{Conclusions}

In summary, we have developed a new approach to fabricate microdents on PS film surfaces via Ouzo effect. A ternary solution of acetone-toluene-water with dissolved PS was designed to achieve sequential evaporation of acetone (good solvent for water) and toluene (poor solvent for water). This leads to the spontaneous formation of water droplets that can act as templates for microdent formation during the precipitation of PS flm from the solution. The footprint diameters of the microdents correspond to those of water droplets. By adjusting water concentration and PS concentration in the ternary solution, the size and density and the depth of microdents can be systematically tuned. The proposed method provides an efficient and simple way to fabricate surface microdents with controlled lateral size and depth, which is important to the related applications. As demonstration, a lubricating surface was fabricated by coating a thin film of silicon oil on the microdent-decorated film that exhibits much improved mobility of a water drop with a low contact angle hysteresis.

\subsection{Appendix}

\subsubsection{Design of the ternary solution}

The principle of the proposed method for microdent fabrication is illustrated in Fig. 8.14. To start with, the mixture of PS in Ouzo solution is first deposited on the substrate (Fig. 8.14(a)). Water droplets are then nucleated (Fig. 8.14(b)). After that, PS film starts to precipitate. The nucleated water droplets act as templates. The precipitated PS only deposits on surface areas that are not covered by water droplets (Fig. 8.14(c)). After water droplets are eventually evaporated, microdents are obtained on the substrate (Fig. 8.14(d)).

From the above analysis, one can see that the key to form surface microdents is to design the ternary aqueous solution. Assuming the ternary aqueous solution consists of solvent A, B, and water. Solvent A is mutually soluble with water. To ensure that water droplets nucleate first, solvent A needs to be have the highest evaporation rate among the three solvents. Moreover, water is insoluble in solvent B. As a result, when solvent A is volatilized, water will nucleate first. Additionally, it is better that water has a higher density than solvent B to make sure water droplets will be deposited onto the substrate other than floating in liquid. 


\section{(a)}
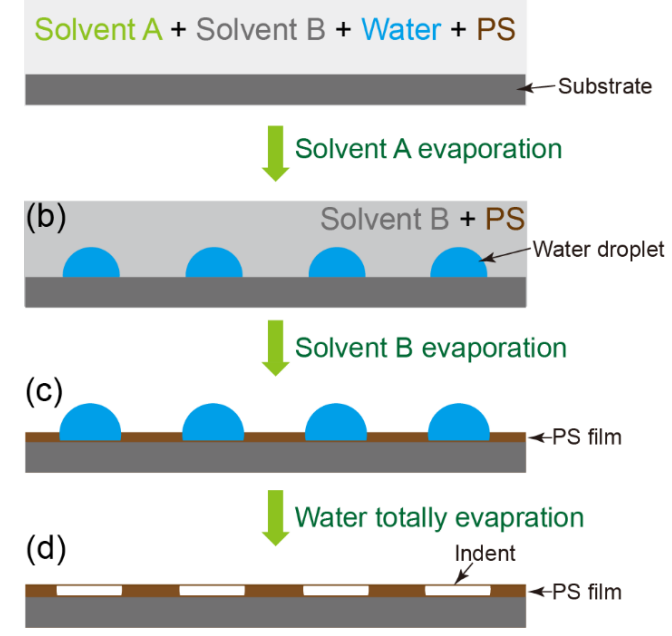

Figure 8.14: Schematic diagram of microdent fabrication via Ouzo effect. (a) Ternary aqueous solution preparation. The ouzo ternary mixture consists of solvent $\mathrm{A}, \mathrm{B}$ and water. The evaporation rate is $\mathrm{A}>\mathrm{B}>$ water. (b) Water droplet nucleation at the solid-liquid interface. (c) Precipitation of PS film with water droplets as templates. (d) Microdents are formed after water droplets fully evaporates.

After water droplets nucleated, PS needs to be deposited on the sample surface. In the designed ternary solution, PS is soluble in solvent B that has higher evaporation rate than water. Therefore, solvent B will start to volatilize after solvent A is volatilized. Accordingly, PS begins to precipitate on the sample surface. The nucleated water droplets can then be taken as templates for microdent formation. As a result, a PS film with generated microdents will be obtained after water droplets are eventually evaporated.

Based on the above conditions, acetone and toluene were selected as solvent $\mathrm{A}$ and solvent $\mathrm{B}$, respectively. Among the three solvents, PS and water are soluble in toluene and acetone, respectively. The physical properties of acetone, toluene, and water are presented in Table 8.1 [241]. From the table, one can see that acetone has the highest evaporation rate, followed by toluene. Water has the lowest evaporation rate. This guarantees sequential nucleation of water droplets and precipitation of PS film. In addition, the density of toluene is lower than that of water, which assures that water droplets can be deposited onto the substrate. 
Table 8.1: Physical properties of water, toluene, and acetone [241].

\begin{tabular}{lccc}
\hline & Water & Toluene & Acetone \\
\hline Evaporation rate $(\mathrm{BuAc}=1.0)$ & 0.36 & 2.0 & 5.6 \\
Relative density & 1 & 0.87 & 0.79 \\
\hline
\end{tabular}

\subsubsection{Microdents decorated surfaces fabricated by different polymers}

To verify the robustness of the proposed method, we applied three other materials to fabricate surface microdents. Figure 8.15 shows the optical images of microdents decorated surfaces generated by low density polyethylene (LDPE), poly(methyl methacrylate) (PMMA), and paraffin. The results indicate that the method is robust and applicable to other polymers.
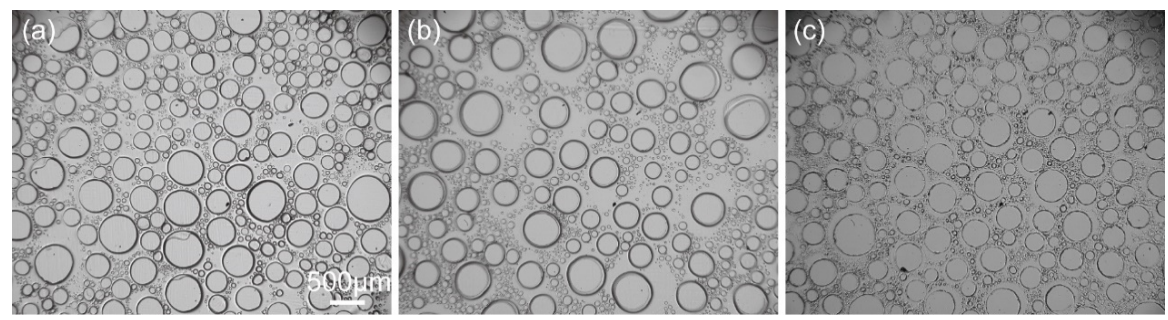

Figure 8.15: Optical images of microdents decorated surfaces fabricated by LDPE (a), PMMA (b) and paraffin (c). 


\section{Chapter 9}

\section{Conclusions and outlook}

In this thesis, we experimentally and theoretically studied plasmonic bubbles, and droplets in multicomponent liquids, focusing on two parts: (I) the nucleation and growth dynamics of the plasmonic bubbles under different ambient pressures and in binary liquids; (II) the nucleation of microdroplets through Ouzo effect, and their applications in the fabrication of surface microstructures.

\section{Part I: Plasmonic bubbles}

In Chapter 2, we studied the nucleation of the initial giant plasmonic bubble in water under different ambient pressures. In the experiment, the ambient pressure was tuned from 1 bar to 9 bar, leading to the change of water boiling point from 100 to $175{ }^{\circ} \mathrm{C}$. The phase transition during initial giant bubble nucleation was revealed. It is found that the delay time of bubble nucleation after turning on the laser is governed by the absolute gas concentration in water, not the ambient pressure. In addition, we demonstrated that the increasing boiling point suppresses the vaporization of the water, resulting in the decreasing of light-vapour conversion efficiency.

In Chapter 3, we investigated the nucleation of initial giant plasmonic bubbles in six different binary liquids. This study revealed the critical liquid parameters for the maximum volume and the delay time $\tau_{d}$ of the initial giant bubbles. We found that at a given energy, the maximum volume of the giant bubble depends on the energy of the liquid required for vaporization $E_{l-v}$.

In Chapter 4, we experimentally and theoretically studied the bouncing dynamics of plasmonic bubbles in binary liquids. The bouncing behavior 
was revealed originating from the competition between thermal and solutal Marangoni forces acting on the nucleating plasmonic bubble. In addition, the dependence of the bouncing frequency on the control parameters was studied. The bouncing frequency increases monotonously with increasing ethanol concentration, while non-monotonously depends the on bubble radius. A theoretical model was proposed to qualitatively describe this dependencies. The theoretical bouncing frequencies agrees well with the experimental observations.

In Chapter 5, we considered the long term behaviour of the plasmonic bubbles in ethanol-water binary liquids. In this study, we found a wiggling bubble followed by a suddenly shrinkage. We experimentally revealed that the bubble was pinned or partially pinned on the laser spot during the wiggling phase in the wiggling stage. When the liquid around the laser spot completely evaporates, the rapid expansion of the three-phase contact line and the sudden shrinkage take place.

\section{Part II: Droplets from the Ouzo effect}

In Chapter 6, we experimentally investigated the formation of surface nanodroplets by the Ouzo effect under well-controlled flow conditions. In this study, a Hele-Shaw cell with a cell height of $100 \mu \mathrm{m}$ was employed, so that the gravitational effects can be negligible. By combining a fluorescence optical microscope and an AFM, we extracted the volume of the produced nanodroplets under different flow rates. The results reveal the underlying mechanism governing the droplet nucleation through the solvent exchange. The mean droplet volume and the probability density function of droplet volumes universally depend on the local $P e$ number.

In Chapter 7, we proposed a simple approach to transfer microdroplets into PS microlenses in a ternary solution system. By adding water into a toluene-ethanol binary liquid, toluene droplets spontaneously nucleate due to the Ouzo effect. The nucleated toluene droplets dissolve PS film coated on the substrate and form PS/toluene mixture droplets. As the toluene in the mixture droplets gradually dissolves into the surrounding solution, PS microlenses were generated on the glass substrate. The size and density of the microlenses were controlled by adjusting PS film thickness and toluene concentration in the toluene/ethanol binary solutions. Moreover, we tuned the contact angle of microlenses from $50^{\circ}$ to $125^{\circ}$ by curing microlens-decorated substrate at different temperature. Finally, it is demonstrated that the obtained microlenses 
can improve the imaging resolution of an optical microscope.

In Chapter 8, we developed an efficient and straightforward method to obtain microdents on PS films through the Ouzo effect. In this method, we designed a ternary solution of acetone-toluene-water with dissolved PS to achieve sequential evaporation of acetone (good solvent for water) and toluene (poor solvent for water). In this system, the acetone first evaporates, leading to droplet nucleation on the substrate. Subsequently, toluene evaporates, and the dissolved PS precipitates from the solution. At the same time, water droplets on the surface act as templates during the precipitation of PS. Finally, after the water evaporates, PS microdents were obtained on the surface. We systematically tuned the size, density and depth of microdents by adjusting water concentration and PS concentration in the ternary solution. As a demonstration, a lubricating surface was fabricated by coating a thin film of silicone oil on the microdent decorated film. It exhibits much-improved mobility of a water drop with a low contact angle hysteresis.

\section{Future directions}

This thesis has addressed several topics related to the plasmonic bubbles and droplets from the Ouzo effect. Many issues are yet to be explored and need further study. Here we list some directions which might be of interest in the future. For plasmonic bubbles, (i) How to experimentally measure the nucleation temperature of the initial giant plasmonic bubble; (ii) What is the mechanism of the oscillating bubble (life phase 2); (iii) A comprehensive numerical model for the bouncing behaviour of the plasmonic bubble. (iv) How to improve light-vapour conversion efficiency or lower the energy threshold of bubble nucleation. (v) Investigating the bubble/droplet motion under more complicated environment. For the droplets from the Ouzo effect: (i) A numerical model to simulate the droplet formation process from Ouzo effect ; (ii) How to trigger the "Ouzo effect" locally by applying additional field, such as temperature field induced by plasmonic effect; (iii) How to improve the controllability of the droplet formation from the Ouzo effect. And many more others. 


\section{References}

[1] E. X. Berry, "Cloud droplet growth by collection", J. Atmos. Sci. 24, 688-701 (1967).

[2] S. Thorpe and P. Humphries, "Bubbles and breaking waves", Nature 283, 463-465 (1980).

[3] Y. Liu and Y. Zhang, "Bubble growth in rhyolitic melt", Earth Planet. Sci. Lett. 181, 251-264 (2000).

[4] J. J. Lyons, M. M. Haney, D. Fee, A. G. Wech, and C. F. Waythomas, "Infrasound from giant bubbles during explosive submarine eruptions", Nat. Geosci. 12, 952-958 (2019).

[5] A. Srivastava, K. Kikuchi, and T. Ishikawa, "The bubble-induced population dynamics of fermenting yeasts", J. R. Soc., Interface 17, 20200735 (2020).

[6] P. Fu, L. Hou, Z. Ren, Z. Zhang, X. Mao, and Y. Yu, "A droplet/wall impact model and simulation of a bipropellant rocket engine", Aerosp. Sci. Technol. 88, 32-39 (2019).

[7] S. Subpiramaniyam, "Outdoor disinfectant sprays for the prevention of COVID-19: Are they safe for the environment?", Sci. Total Environ. 759, 144289 (2021).

[8] C. Williams, "Ink-jet printers go beyond paper", Phys. World 19, 24-29 (2006).

[9] G. Barker, B. Jefferson, and S. Judd, "The control of bubble size in carbonated beverages", Chem. Eng. Sci. 57, 565-573 (2002). 
[10] P. Day, A. Manz, and Y. Zhang, Microdroplet technology: principles and emerging applications in biology and chemistry (Springer Science \& Business Media) (2012).

[11] K. L. Chong, C. S. Ng, N. Hori, R. Yang, R. Verzicco, and D. Lohse, "Extended Lifetime of Respiratory Droplets in a Turbulent Vapor Puff and Its Implications on Airborne Disease Transmission", Phys. Rev. Lett. 126, 034502 (2021).

[12] X. Liang, H. Tan, Z. Fu, and S. Y. Chou, "Air bubble formation and dissolution in dispensing nanoimprint lithography", Nanotechnol. 18, 025303 (2007).

[13] R. Patella and J. Reboud, "A new approach to evaluate the cavitation erosion power", J. Fluids Eng. 120, 335-344 (1998).

[14] M. E. Cates and E. Tjhung, "Theories of binary fluid mixtures: from phase-separation kinetics to active emulsions", J. Fluid Mech. 836, P1 (2018).

[15] Y. T. Maeda, A. Buguin, and A. Libchaber, "Thermal separation: interplay between the soret effect and entropic force gradient", Phys. Rev. Lett. 107, 038301 (2011).

[16] L. Scriven and C. Sternling, "The Marangoni effects", Nature 187, 186188 (1960).

[17] J. R. Christy, Y. Hamamoto, and K. Sefiane, "Flow transition within an evaporating binary mixture sessile drop", Phys. Rev. Lett. 106, 205701 (2011).

[18] N. J. Cira, A. Benusiglio, and M. Prakash, "Vapour-mediated sensing and motility in two-component droplets", Nature 519, 446-450 (2015).

[19] H. Tan, C. Diddens, P. Lv, J. G. M. Kuerten, X. Zhang, and D. Lohse, "Evaporation-triggered microdroplet nucleation and the four life phases of an evaporating ouzo drop", Proc. Nat. Acad. Sci. 113, 8642-8647 (2016).

[20] S. Vitale and J. Katz, "Liquid droplet dispersions formed by homogeneous liquid-liquid nucleation: "The ouzo effect"”, Langmuir 19, 41054110 (2003). 
[21] J. R. Adleman, D. A. Boyd, D. G. Goodwin, and D. Psaltis, "Heterogenous catalysis mediated by plasmon heating", Nano Letters 9, 4417-4423 (2009).

[22] G. Baffou and R. Quidant, "Nanoplasmonics for chemistry", Chem. Soc. Rev. 43, 3898-3907 (2014).

[23] O. Neumann, C. Feronti, A. D. Neumann, A. Dong, K. Schell, B. Lu, E. Kim, M. Quinn, S. Thompson, N. Grady, P. Nordlander, M. Oden, and N. J. Halas, "Compact solar autoclave based on steam generation using broadband light-harvesting nanoparticles", Proc. Natl. Acad. Sci. U. S. A. 110, 11677-11681 (2013).

[24] S. V. Boriskina, H. Ghasemi, and G. Chen, "Plasmonic materials for energy: From physics to applications", Mater. Today 16, 375-386 (2013).

[25] H. Ghasemi, G. Ni, A. M. Marconnet, J. Loomis, S. Yerci, N. Miljkovic, and G. Chen, "Solar steam generation by heat localization", Nat. Commun. 5, 1-7 (2014).

[26] X. Wang, Y. He, X. Liu, G. Cheng, and J. Zhu, "Solar steam generation through bio-inspired interface heating of broadband-absorbing plasmonic membranes", Appl. Energy 195, 414-425 (2017).

[27] J. Shao, M. Xuan, L. Dai, T. Si, J. Li, and Q. He, "Near-infraredactivated nanocalorifiers in microcapsules: Vapor bubble generation for In Vivo enhanced cancer therapy", Angew. Chem., Int. Ed. 54, 12782 12787 (2015).

[28] J. Lombard, T. Biben, and S. Merabia, "Nanobubbles around plasmonic nanoparticles: Thermodynamic analysis", Phys. Rev. E 91, 043007 (2015).

[29] Y. Ren, Q. Chen, H. Qi, L. Ruan, and J. Dai, "Phase transition induced by localized surface plasmon resonance of nanoparticle assemblies", Int. J. Heat Mass Transfer 127, 244-252 (2018).

[30] Y. Wang, M. E. Zaytsev, G. Lajoinie, H. L. The, J. C. T. Eijkel, A. van den Berg, B. M. Weckhuysen, X. Zhang, H. J. W. Zandvliet, and D. Lohse, "Giant and explosive plasmonic bubbles by delayed nucleation", Proc. Natl. Acad. Sci. U. S. A. 115, 7676-7681 (2018). 
[31] M. E. Zaytsev, G. Lajoinie, Y. Wang, D. Lohse, H. J. W. Zandvliet, and X. Zhang, "Plasmonic Bubbles in n-Alkanes", J. Phys. Chem. C 122, 28375-28381 (2018).

[32] X. Li, Y. Wang, M. E. Zaytsev, G. Lajoinie, H. Le The, J. G. Bomer, J. C. Eijkel, H. J. Zandvliet, X. Zhang, and D. Lohse, "Plasmonic bubble nucleation and growth in water: effect of dissolved air", J. Phys. Chem. C 123, 23586-23593 (2019).

[33] M. E. Zaytsev, Y. Wang, Y. Zhang, G. Lajoinie, X. Zhang, A. Prosperetti, H. J. Zandvliet, and D. Lohse, "Gas-vapor interplay in plasmonic bubble shrinkage", J. Phys. Chem. C 124, 5861-5869 (2020).

[34] E. Lukianova-Hleb, Y. Hu, L. Latterini, L. Tarpani, S. Lee, R. A. Drezek, J. H. Hafner, and D. O. Lapotko, "Plasmonic nanobubbles as transient vapor nanobubbles generated around plasmonic nanoparticles", ACS Nano 4, 2109-2123 (2010).

[35] D. Wang, P. Cheng, and X. Quan, "Photothermal nanobubble nucleation on a plasmonic nanoparticle: A 3D lattice Boltzmann simulation", Int. J. Heat Mass Transfer 140, 786-797 (2019).

[36] Y. Zhang and A. Prosperetti, "Dynamics, heat and mass transfer of a plasmonic bubble on a solid surface", Int. J. Heat Mass Transfer 167 (2021).

[37] A. Mendez-Vilas, A. B. Jodar-Reyes, and M. L. Gonzalez-Martin, "Ultrasmall Liquid Droplets on Solid Surfaces: Production, Imaging, and Relevance for Current Wetting Research", Small 5, 1366-1390 (2009).

[38] D. T. Chiu and R. M. Lorenz, "Chemistry and Biology in Femtoliter and Picoliter Volume Droplets", Acc. Chem. Res. 42, 649-658 (2009).

[39] J. Shemesh, T. Ben Arye, J. Avesar, J. H. Kang, A. Fine, M. Super, A. Meller, D. E. Ingber, and S. Levenberg, "Stationary nanoliter droplet array with a substrate of choice for single adherent/nonadherent cell incubation and analysis", Proc. Natl. Acad. Sci. U. S. A. 111, 1129311298 (2014).

[40] B. Dyett, Q. Zhang, Q. Xu, X. Wang, and X. Zhang, "Extraordinary focusing effect of surface nanolenses in total internal reflection mode", ACS Cent. Sci. 4, 1511-1519 (2018). 
[41] R. U. Meckenstock, N. F. Von, C. Stumpp, T. Lueders, A. M. Himmelberg, N. Hertkorn, P. Schmitt-Kopplin, M. Harir, R. Hosein, and S. Haque, "Oil biodegradation. water droplets in oil are microhabitats for microbial life", Science 345, 673-676 (2014).

[42] H. Yang, S. Peng, X. Hao, T. A. Smith, G. G. Qiao, and X. H. Zhang, "Surfactant-mediated formation of polymeric microlenses from interfacial microdroplets", Soft Matter 10, 957 (2014).

[43] X. Zhang, Z. Lu, H. Tan, L. Bao, Y. He, C. Sun, and D. Lohse, "Formation of surface nanodroplets under controlled flow conditions", Proc. Natl. Acad. Sci. U. S. A. 112, 9253-9257 (2015).

[44] J. Aubry, F. Ganachaud, J.-P. C. Addad, and B. Cabane, "Nanoprecipitation of Polymethylmethacrylate by Solvent Shifting: 1. Boundaries", Langmuir 25, 1970-1979 (2009).

[45] E. Scholten, E. van der Linden, and H. This, "The life of an aniseflavored alcoholic beverage: Does its stability cloud or confirm theory?", Langmuir 24, 1701-1706 (2008).

[46] X. Zhang and W. Ducker, "Formation of interfacial nanodroplets through changes in solvent quality", Langmuir 23, 12478-12480 (2007).

[47] L. Bao, A. R. Rezk, L. Y. Yeo, and X. Zhang, "Highly ordered arrays of femtoliter surface droplets", Small 11, 4850-4855 (2015).

[48] H. Yu, Z. Lu, D. Lohse, and X. Zhang, "Gravitational effect on the formation of surface nanodroplets", Langmuir 31, 12628-12634 (2015).

[49] Z. Lu, S. Peng, and X. Zhang, "Influence of solution composition on the formation of surface nanodroplets by solvent exchange", Langmuir 32, 1700-1706 (2016).

[50] M. T. Carlson, A. J. Green, and H. H. Richardson, "Superheating water by cw excitation of gold nanodots", Nano Lett. 12, 1534-1537 (2012).

[51] Z. Fang, Y. R. Zhen, O. Neumann, A. Polman, F. J. Garcia de Abajo, P. Nordlander, and N. J. Halas, "Evolution of light-induced vapor generation at a liquid-immersed metallic nanoparticle", Nano Lett. 13, 1736$1742(2013)$. 
[52] S. Baral, A. J. Green, M. Y. Livshits, A. O. Govorov, and H. H. Richardson, "Comparison of vapor formation of water at the solid/water interface to colloidal solutions using optically excited gold nanostructures", ACS NANO 8, 1439-1448 (2014).

[53] X. Liu, L. Bao, M. Dipalo, F. De Angelis, and X. Zhang, "Formation and dissolution of microbubbles on highly-ordered plasmonic nanopillar arrays", Sci. Rep. 5, 18515 (2015).

[54] D. Lapotko, "Plasmonic nanoparticle-generated photothermal bubbles and their biomedical applications", Nanomedicine 4, 813-845 (2009).

[55] Q. Fan, W. Hu, and A. T. Ohta, "Laser-induced microbubble poration of localized single cells", Lab Chip 14, 1572-1578 (2014).

[56] L. Lin, X. Peng, Z. Mao, W. Li, M. N. Yogeesh, B. B. Rajeeva, E. P. Perillo, A. K. Dunn, D. Akinwande, and Y. Zheng, "Bubble-pen lithography", Nano Lett. 16, 701-708 (2016).

[57] Y. Xie and C. Zhao, "An optothermally generated surface bubble and its applications", Nanoscale 9, 6622-6631 (2017).

[58] F. Tantussi, G. C. Messina, R. Capozza, M. Dipalo, L. Lovato, and F. De Angelis, "Long-range capture and delivery of water-dispersed nano-objects by microbubbles generated on 3D plasmonic surfaces", ACS Nano 12, 4116-4122 (2018).

[59] H. Jin, G. Lin, L. Bai, A. Zeiny, and D. Wen, "Steam generation in a nanoparticle-based solar receiver", Nano Energy 28, 397-406 (2016).

[60] L. Zhou, S. Zhuang, C. He, Y. Tan, Z. Wang, and J. Zhu, "Self-assembled spectrum selective plasmonic absorbers with tunable bandwidth for solar energy conversion", Nano Energy 32, 195-200 (2017).

[61] L. Zhou, Y. Tan, J. Wang, W. Xu, Y. Yuan, W. Cai, S. Zhu, and J. Zhu, "3D self-assembly of aluminium nanoparticles for plasmon-enhanced solar desalination", Nat. Photonics 10, 393-398 (2016).

[62] Y. Wang, M. E. Zaytsev, H. L. The, J. C. T. Eijkel, H. J. W. Zandvliet, X. Zhang, and D. Lohse, "Vapor and gas-bubble growth dynamics around laser-irradiated, water-immersed plasmonic nanoparticles", ACS Nano 11, 2045-2051 (2017). 
[63] X. Li, Y. Wang, B. Zeng, M. Detert, A. Prosperetti, H. J. W. Zandvliet, and D. Lohse, "Plasmonic Microbubble Dynamics in Binary Liquids", J. Phys. Chem. Lett. 11, 8631-8637 (2020).

[64] H. L. The, E. Berenschot, R. M. Tiggelaar, N. R. Tas, A. van den Berg, and J. C. T. Eijkel, "Shrinkage control of photoresist for large-area fabrication of sub-30 nm periodic nanocolumns", Adv. Mater. Technol. 2, 1600238 (2017).

[65] Y. Wang, T. Lu, X. Li, and H. Wang, "Automated image segmentationassisted flattening of atomic force microscopy images", Beilstein J. Nanotechnol. 9, 975-985 (2018).

[66] Y. Wang, T. Lu, X. Li, S. Ren, and S. Bi, "Robust nanobubble and nanodroplet segmentation in atomic force microscope images using the spherical Hough transform", Beilstein J. Nanotechnol. 8, 2572-2582 (2017).

[67] Y. Wang, Z. Zhang, H. Wang, and S. Bi, "Segmentation of the clustered cells with optimized boundary detection in negative phase contrast images", PLoS One 10 (2015).

[68] V. P. Skripov and P. A. Pavlov, "Explosive boiling of liquids and fluctuation nucleus formation", High Temp. 8, 833-839 (1970).

[69] M. Blander and J. L. Katz, "Bubble nucleation in liquids", AIChE J. 21, 833-848 (1975).

[70] S. E. Puchinskis and P. V. Skripov, "The attainable superheat: From simple to polymeric liquids", Int. J. Thermophys. 22, 1755-1768 (2001).

[71] J. Lombard, T. Biben, and S. Merabia, "Kinetics of nanobubble heneration around overheated nanoparticles", Phys. Rev. Lett. 112, 105701 (2014).

[72] G. V. Hartland, L. V. Besteiro, P. Johns, and A. O. Govorov, "What' s so hot about electrons in metal nanoparticles?", ACS Energy Lett. 2, 1641-1653 (2017).

[73] E. in Chief: David R. Lide, Handbook of Chemistry and Physics, 86th edition (Taylor and Francis) (2005). 
[74] N. Xu, X. Hu, W. Xu, X. Li, L. Zhou, S. Zhu, and J. Zhu, "Mushrooms as efficient solar steam-generation devices", Adv. Mater. 29, 1606762 (2017).

[75] L. Zhou, X. Li, G. W. Ni, S. Zhu, and J. Zhu, "The revival of thermal utilization from the Sun: interfacial solar vapor generation", Natl. Sci. Rev. 6, 562-578 (2019).

[76] X. Wang, X. Li, G. Liu, J. Li, X. Hu, N. Xu, W. Zhao, B. Zhu, and $\mathrm{J}$. Zhu, "An interfacial solar heating assisted liquid sorbent atmospheric water generator", Angew. Chem., Int. Ed. 58, 12054-12058 (2019).

[77] G. Baffou, J. Polleux, H. Rigneault, and S. Monneret, "Super-heating and micro-bubble generation around plasmonic nanoparticles under $\mathrm{cw}$ illumination", J. Phys. Chem. C 118, 4890-4898 (2014).

[78] X. Liu, L. Bao, M. Dipalo, F. De Angelis, and X. Zhang, "Formation and dissolution of microbubbles on highly-ordered plasmonic nanopillar arrays", Sci. Rep. 5, 18515 (2015).

[79] L. Hirsch, R. Stafford, J. Bankson, S. Sershen, B. Rivera, R. Price, J. Hazle, N. Halas, and J. West, "Nanoshell-mediated Near-Infrared Thermal Therapy of Tumors under Magnetic Resonance Guidance", Proc. Natl. Acad. Sci. U. S. A. 100, 13549-13554 (2003).

[80] E. Y. Lukianova-Hleb, X. Ren, J. A. Zasadzinski, X. Wu, and D. O. Lapotko, "Plasmonic nanobubbles enhance efficacy and selectivity of chemotherapy against drug-resistant cancer cells", Adv. Mater. 24, 3831-3837 (2012).

[81] G. Baffou and R. Quidant, "Thermo-plasmonics: Using metallic nanostructures as nano-sources of heat", Laser Photonics Rev. 7, 171-187 (2013).

[82] H.-L. Liu, C.-H. Fan, C.-Y. Ting, and C.-K. Yeh, "Combining Microbubbles and Ultrasound for Drug Delivery to Brain Tumors: Current Progress and Overview", Theranostics 4, 432-444 (2014).

[83] L. Minai, M. Hamra, and D. Yelin, "Plasmonic targeting of cancer cells in a three-dimensional natural hydrogel", Nanoscale 10, 17807-17813 (2018). 
[84] X. Zhang, Y. Wang, S. Watanabe, M. H. Uddin, and D. Li, "Evaporation-induced flattening and self-assembly of chemically converted graphene on a solid surface", Soft Matter 7, 8745-8748 (2011).

[85] P. Christopher, H. Xin, and S. Linic, "Visible-light-enhanced catalytic oxidation reactions on plasmonic silver nanostructures", Nat. Chem. 3, 467 (2011).

[86] Battino, Rubin and Rettich, Timothy R and Tominaga, Toshihiro, "The solubility of nitrogen and air in liquids", J. Phys. Chem. Ref. Data 13, 563-600 (1984).

[87] Kretschmer, Carl B and Nowakowska, Janina and Wiebe, Richard, "Solubility of oxygen and nitrogen in organic solvents from -25 to $50{ }^{\circ} \mathrm{C}$ ", Ind. Eng. Chem. 38, 506-509 (1946).

[88] Tokunaga, Junji, "Solubilities of oxygen, nitrogen, and carbon dioxide in aqueous alcohol solutions", J. Chem. Eng. Data 20, 41-46 (1975).

[89] Tsuji, $\mathrm{K}$ and Ichikawa, $\mathrm{K}$ and Yamamoto, $\mathrm{H}$ and Tokunaga, J, "Solubilities of oxygen and nitrogen in acetone-water mixed solvent", Kagaku Kogaku Ronbunshu 13, 825-830 (1987).

[90] "National institute of standards and technology. nist chemistry webbook, nist standard reference database number 69 . https://webbook.nist.gov/chemistry (accessed apr 8, 2019)", .

[91] V. P. Skripov and J. L. Katz, "Metastable liquids", Phys. Today 28, 57 (1975).

[92] Y. Mori, K. Hijikata, and T. Nagatani, "Effect of dissolved gas on bubble nucleation", Int. J. Heat Mass Transfer 19, 1153-1159 (1976).

[93] "Dortmund data bank. ddbs. http://www.ddbst.com/en/eed/vle/vleindex.php (accessed apr 1, 2021)", .

[94] G. Vazquez, E. Alvarez, and J. M. Navaza, "Surface tension of alcohol + water from 20 to $50{ }^{\circ}$ c", J. Chem. Eng. Data 40, 611-614 (1995).

[95] D. Lohse, "Bubble puzzles: From fundamentals to applications", Phys. Rev. Fluids 3, 110504 (2018). 
[96] A. Bergeon, D. Henry, H. Benhadid, and L. S. Tuckerman, "Marangoni convection in binary mixtures with soret effect", J. Fluid Mech. 375, 143-177 (1998).

[97] J. Liu and G. Ahlers, "Spiral-defect chaos in rayleigh-bénard convection with small prandtl numbers", Phys. Rev. Lett. 77, 3126 (1996).

[98] D. Dedovets, C. Monteux, and S. Deville, "Five-dimensional imaging of freezing emulsions with solute effects", Science 360, 303-306 (2018).

[99] S. Deville, Freezing colloids: observations, principles, control, and use: applications in materials science, life science, earth science, food science, and engineering (Springer, Berlin) (2017).

[100] C. Diddens, H. Tan, P. Lv, M. Versluis, J. G. M. Kuerten, X. Zhang, and D. Lohse, "Evaporating pure, binary and ternary droplets: thermal effects and axial symmetry breaking", J. Fluid Mech. 823, 470-497 (2017).

[101] C. Diddens, J. Kuerten, C. van der Geld, and H. Wijshoff, "Modeling the evaporation of sessile multi-component droplets", J. Colloid Inferf. Sci. 487, 426-436 (2017).

[102] H. Kim and H. A. Stone, "Direct measurement of selective evaporation of binary mixture droplets by dissolving materials", J. Fluid Mech. 850, 769-783 (2018).

[103] Y. Li, P. Lv, C. Diddens, H. Tan, H. Wijshoff, M. Versluis, and D. Lohse, "Evaporation-triggered segregation of sessile binary droplets", Phys. Rev. Lett. 120, 224501 (2018).

[104] A. Edwards, P. Atkinson, C. Cheung, H. Liang, D. Fairhurst, and F. Ouali, "Density-driven flows in evaporating binary liquid droplets", Phys. Rev. Lett. 121, 184501 (2018).

[105] Y. Li, C. Diddens, P. Lv, H. Wijshoff, M. Versluis, and D. Lohse, "Gravitational effect in evaporating binary microdroplets", Phys. Rev. Lett. 122, 114501 (2019).

[106] S. Chu and A. Prosperetti, "History effects on the gas exchange between a bubble and a liquid", Phys. Rev. Fluids 1, 064202 (2016). 
[107] E. Dietrich, M. Rump, P. Lv, E. S. Kooij, H. J. W. Zandvliet, and D. Lohse, "Segregation in dissolving binary-component sessile droplets", J. Fluid Mech. 812, 349-369 (2017).

[108] S. Maheshwari, M. Van Der Hoef, A. Prosperetti, and D. Lohse, "Molecular dynamics study of multicomponent droplet dissolution in a sparingly miscible liquid", J. Fluid Mech. 833, 54-69 (2017).

[109] J. M. E. Escobar, J. Nieland, A. van Houselt, X. Zhang, and D. Lohse, "Marangoni puffs: dramatically enhanced dissolution of droplets with an entrapped bubble", Soft Matter 16, 4520-4527 (2020).

[110] V. K. Dhir, "Boiling heat transfer", Annu. Rev. Fluid Mech. 30, 365-401 (1998).

[111] A. Bashkatov, S. S. Hossain, X. Yang, G. Mutschke, and K. Eckert, "Oscillating hydrogen bubbles at pt microelectrodes", Phys. Rev. Lett. 123, 214503 (2019).

[112] X. Yang, D. Baczyzmalski, C. Cierpka, G. Mutschke, and K. Eckert, "Marangoni convection at electrogenerated hydrogen bubbles", Phys. Chem. Chem. Phys. 20, 11542-11548 (2018).

[113] M. Manjare, B. Yang, and Y.-P. Zhao, "Bubble driven quasioscillatory translational motion of catalytic micromotors", Phys. Rev. Lett. 109, 128305 (2012).

[114] P. Lv, H. Le The, J. Eijkel, A. Van den Berg, X. Zhang, and D. Lohse, "Growth and detachment of oxygen bubbles induced by gold-catalyzed decomposition of hydrogen peroxide", J. Phys. Chem. C 121, 20769 20776 (2017).

[115] E. Lauga and T. R. Powers, "The hydrodynamics of swimming microorganisms", Reports on Progress in Physics 72, 096601 (2009).

[116] C. C. Maass, C. Krüger, S. Herminghaus, and C. Bahr, "Swimming droplets", Annu. Rev. Cond. Matter Phys. 7, 171-193 (2016).

[117] Z. Izri, M. N. Van Der Linden, S. Michelin, and O. Dauchot, "Selfpropulsion of pure water droplets by spontaneous Marangoni-stressdriven motion", Phys. Rev. Lett. 113, 248302 (2014). 
[118] S. Michelin and E. Lauga, "Phoretic self-propulsion at finite Péclet numbers", J. Fluid Mech. 747, 572-604 (2014).

[119] J. L. Moran and J. D. Posner, "Phoretic self-propulsion", Annu. Rev. Fluid Mech. 49, 511-540 (2017).

[120] R. Golestanian, T. B. Liverpool, and A. Ajdari, "Propulsion of a molecular machine by asymmetric distribution of reaction products", Phys. Rev. Lett. 94, 220801 (2005).

[121] A. Mani and K. M. Wang, "Electroconvection near electrochemical interfaces: Experiments, modeling, and computation", Annu. Rev. Fluid Mech. 52, 509-529 (2020).

[122] V. G. Levich, Physicochemical hydrodynamics (Prentice Hall, Englewood Cliffs) (1962).

[123] A. De Wit, "Chemo-hydrodynamic patterns and instabilities", Annu. Rev. Fluid Mech. 52, 531-555 (2020).

[124] D. Lohse and X. Zhang, "Physicochemical hydrodynamics of droplets out of equilibrium", Nature Rev. Phys. 2, 426-443 (2020).

[125] Y. Li, C. Diddens, A. Prosperetti, K. L. Chong, X. Zhang, and D. Lohse, "Bouncing oil droplet in a stratified liquid and its sudden death", Phys. Rev. Lett. 122, 154502 (2019).

[126] K. Schwarzenberger, S. Aland, H. Domnick, S. Odenbach, and K. Eckert, "Relaxation oscillations of solutal marangoni convection at curved interfaces", Colloids Surf., A 481, 633-643 (2015).

[127] M. Detert, B. Zeng, Y. Wang, H. Le The, H. J. Zandvliet, and D. Lohse, "Plasmonic bubble nucleation in binary liquids", J. Phys. Chem. C 124, 2591-2597 (2020).

[128] S. Y. Emelianov, P.-C. Li, and M. O'Donnell, "Photoacoustics for molecular imaging and therapy", Phys. Today 62, 34-39 (2009).

[129] Y. Liu and S. J. Dillon, "In situ observation of electrolytic $h_{2}$ evolution adjacent to gold cathodes", Chem. Comm. 50, 1761-1763 (2014).

[130] A. Prosperetti, "Vapor bubbles", Annu. Rev. Fluid Mech. 49, 221-248 (2017). 
[131] J. Magnaudet and D. Legendre, "The viscous drag force on a spherical bubble with a time-dependent radius", Phys. Fluids 10, 550-554 (1998).

[132] J. Magnaudet and I. Eames, "The motion of high-Reynolds number bubbles in inhomogeneous flows", Annu. Rev. Fluid Mech. 32, 659-708 (2000).

[133] J. M. Rensen, D. Bosman, J. Magnaudet, C. D. Ohl, A. Prosperetti, R. Tögel, M. Versluis, and D. Lohse, "Spiraling bubbles: How acoustic and hydrodynamic forces compete", Phys. Rev. Lett. 86, 4819-4822 (2001).

[134] R. Toegel, S. Luther, and D. Lohse, "Viscosity destablizes sonoluminescing bubbles", Phys. Rev. Lett. 96, 114301 (2006).

[135] Y. Li, C. Diddens, A. Prosperetti, and D. Lohse, "From levitating to bouncing: Marangoni instability of a drop in a stratified liquid", Phys. Rev. Lett. 126, 124502 (2021).

[136] H. Schlichting, Boundary layer theory, 7th edition (McGraw Hill, New York) (1979).

[137] K. Kurihara, M. Nakamichi, and K. Kojima, "Isobaric vapor-liquid equilibria for methanol+ ethanol+ water and the three constituent binary systems", J. Chem. Eng. Data 38, 446-449 (1993).

[138] I. S. Khattab, F. Bandarkar, M. A. A. Fakhree, and A. Jouyban, "Density, viscosity, and surface tension of water+ ethanol mixtures from 293 to 323k", Korean J. Chem. Eng. 29, 812-817 (2012).

[139] C. Zhao, Y. Xie, Z. Mao, Y. Zhao, J. Rufo, S. Yang, F. Guo, J. D. Mai, and T. J. Huang, "Theory and experiment on particle trapping and manipulation via optothermally generated bubbles", Lab Chip 14, 384-391 (2014).

[140] Y. Xie and C. Zhao, "An optothermally generated surface bubble and its applications", Nanoscale 9, 6622-6631 (2017).

[141] B. P. Dyett, M. Li, H. Zhao, and X. Zhang, "Plasmonic nanobubbles in "armored" surface nanodroplets", J. Phys. Chem. C 123, 29866-29874 (2019). 
[142] J. Lombard, T. Biben, and S. Merabia, "Ballistic heat transport in laser generated nano-bubbles", Nanoscale 8, 14870-14876 (2016).

[143] S. Maheshwari, M. van der Hoef, A. Prosperetti, and D. Lohse, "Dynamics of formation of a vapor nanobubble around a heated nanoparticle", J. Phys. Chem. C 122, 20571-20580 (2018).

[144] Y. Li, C. Diddens, A. Prosperetti, K. L. Chong, X. Zhang, and D. Lohse, "Bouncing oil droplet in a sratified liquid and its sudden death", Phys. Rev. Lett. 122, 154502 (2019).

[145] W. L. Tong, M. K. Tan, J. K. Chin, K. S. Ong, and Y. M. Hung, "Coupled effects of hydrophobic layer and vibration on thermal efficiency of two-phase closed thermosyphons", RSC Adv. 5, 10332-10340 (2015).

[146] H. Oguz and A. Prosperetti, "Dynamics of bubble-growth and detachment from a needle", J. Fluid Mech. 257, 111-145 (1993).

[147] P. Lv, H. Le The, J. Eijkel, A. Van den Berg, X. Zhang, and D. Lohse, "Growth and detachment of oxygen bubbles induced by gold-catalyzed decomposition of hydrogen peroxide", J. Phys. Chem. C 121, 2076920776 (2017).

[148] M. A. Kalbassi and M. W. Biddulph, "Surface tension of mixtures at their boiling points", J. Chem. Eng. Data 33, 473-476 (1988).

[149] Y. Yonemoto, S. Suzuki, S. Uenomachi, and T. Kunugi, "Sliding behaviour of water-ethanol mixture droplets on inclined low-surface-energy solid", Int. J. Heat Mass Transfer 120, 1315-1324 (2018).

[150] C. Liu, E. Bonaccurso, and H.-J. Butt, "Evaporation of sessile water/ethanol drops in a controlled environment", Phys. Chem. Chem. Phys. 10, 7150-7157 (2008).

[151] A. K. H. Cheng, D. M. Soolaman, and H.-Z. Yu, "Evaporation of microdroplets of ethanol-water mixtures on gold surfaces modified with self-assembled monolayers", J. Phys. Chem. B 110, 11267-11271 (2006).

[152] C. H. Ooi, E. Bormashenko, A. V. Nguyen, G. M. Evans, D. V. Dao, and N.-T. Nguyen, "Evaporation of ethanol-water binary mixture sessile liquid marbles", Langmuir 32, 6097-6104 (2016). 
[153] K. Sefiane, L. Tadrist, and M. Douglas, "Experimental study of evaporating water-ethanol mixture sessile drop: influence of concentration", Int. J. Heat Mass Transf. 46, 4527-4534 (2003).

[154] Z. Wang, X.-F. Peng, A. S. Mujumdar, A. Su, and D.-J. Lee, "Evaporation of ethanol-water mixture drop on horizontal substrate", Dry. Technol. 26, 806-810 (2008).

[155] Y. Yonemoto and T. Kunugi, "Experimental and theoretical investigation of contact-angle variation for water-ethanol mixture droplets on a low-surface-energy solid", Int. J. Heat Mass Transf. 96, 614-626 (2016).

[156] D. Lohse and X. Zhang, "Surface nanobubbles and nanodroplets", Rev. Mod. Phys. 87, 981-1035 (2015).

[157] M. E. R. Shanahan, "Simple theory of stick-slip wetting hysteresis", Langmuir 11, 1041-1043 (1995).

[158] J. M. Stauber, S. K. Wilson, B. R. Duffy, and K. Sefiane, "On the lifetimes of evaporating droplets", J. Fluid Mech. 744, R2 (2014).

[159] J. M. E. Escobar, E. Dietrich, S. Arscott, H. J. W. Zandvliet, X. Zhang, and D. Lohse, "Zipping-depinning: dissolution of droplets on micropatterned concentric rings", Langmuir 34, 5396-5402 (2018).

[160] J. M. E. Escobar, D. Garcia-Gonzalez, I. Devic, X. Zhang, and D. Lohse, "Morphology of evaporating sessile microdroplets on lyophilic elliptical patches", Langmuir 35, 2099-2105 (2019).

[161] F. Barr-David and B. F. Dodge, "Vapor-liquid equilibrium at high pressures. the systems ethanol-water and 2-propanol-water.", J. Chem. Eng. Data 4, 107-121 (1959).

[162] B. E. Wyslouzil and J. Woelk, "Overview: Homogeneous nucleation from the vapor phase-The experimental science", J. Chem. Phys. 145, 26-31 (2016).

[163] X. H. Zhang and W. Ducker, "Interfacial oil droplets", Langmuir 24, 110-115 (2008).

[164] Q. Xiao, Y. Liu, Z. Guo, Z. Liu, D. Lohse, and X. Zhang, "Solvent exchange leading to nanobubble nucleation: a molecular dynamics study", Langmuir 33, 8090-8096 (2017). 
[165] C. Xu, S. Peng, G. G. Qiao, V. Gutowski, D. Lohse, and X. Zhang, "Nanobubble formation on a warmer substrate.", Soft Matter 10, 78577864 (2014).

[166] H. Xu and X. Zhang, "Formation, characterization and stability of oil nanodroplets on immersed substrates", Adv. Colloid Interface Sci. 224, $17-32(2015)$.

[167] H. Zhang, S. Chen, Z. Guo, Y. Liu, F. Bresme, and X. Zhang, "Contact line pinning effects influence determination of the line tension of droplets adsorbed on substrates", J. Phys. Chem. C 122, 17184-17189 (2018).

[168] C. A. Strulson, R. C. Molden, C. D. Keating, and P. C. Bevilacqua, "RNA catalysis through compartmentalization", Nat. Chem. 4, 941-946 (2012).

[169] Y. Chen, M. Elshobaki, R. Gebhardt, S. Bergeson, M. Noack, J.-M. Park, A. C. Hillier, K.-M. Ho, R. Biswas, and S. Chaudhary, "Reducing optical losses in organic solar cells using microlens arrays: theoretical and experimental investigation of microlens dimensions", Phys. Chem. Chem. Phys. 17, 3723-3730 (2015).

[170] S. Peng, C. Xu, T. C. Hughes, and X. Zhang, "From nanodroplets by the ouzo effect to interfacial nanolenses", Langmuir 30, 12270-12277 (2014).

[171] X. Zhang, X. Wei, and W. Ducker, "Formation of nanodents by deposition of nanodroplets at the polymer-liquid interface", Langmuir $\mathbf{2 6}$, 4776-4781 (2010).

[172] S. Darwich, K. Mougin, L. Vidal, E. Gnecco, and H. Haidara, "Nanobubble and nanodroplet template growth of particle nanorings versus nanoholes in drying nanofluids and polymer films", Nanoscale 3, 12111217 (2011).

[173] A. Ma, J. Xu, X. Zhang, B. Zhang, D. Wang, and H. Xu, "Interfacial nanodroplets guided construction of hierarchical au, au-pt, and au-pd particles as excellent catalysts", Sci. Rep. 4, 4849 (2014).

[174] M. Duocastella, C. Florian, P. Serra, and A. Diaspro, "Sub-wavelength laser nanopatterning using droplet lenses", Sci. Rep. 5, 16199 (2015). 
[175] R. Zhang, W. Liao, Y. Sun, J. Y. Y. Heng, and Z. Yang, "Investigating the role of glass and quartz substrates on the formation of interfacial droplets", J. Phys. Chem. C 123, 1151-1159 (2019).

[176] S.-W. Hung and J. Shiomi, "Dynamic wetting of nanodroplets on smooth and patterned graphene-coated surface", J. Phys. Chem. C 122, 84238429 (2018).

[177] S. Gao, Q. Liao, W. Liu, and Z. Liu, "Self-removal of multiple and multisize coalescing nanodroplets on nanostructured surfaces", J. Phys. Chem. C 122, 20521-20526 (2018).

[178] X. Zhang, H. Lhuissier, C. Sun, and D. Lohse, "Surface nanobubbles nucleate microdroplets", Phys. Rev. Lett. 112, 144503 (2014).

[179] S. Peng, V. Spandan, R. Verzicco, D. Lohse, and X. H. Zhang, "Growth dynamics of microbubbles on microcavity arrays by solvent exchange", J. Colloidal \& Interface Sci. 532, 103-111 (2018).

[180] S. Peng, T. L. Mega, and X. Zhang, "Collective effects in microbubble growth by solvent exchange", Langmuir 32, 11265-11272 (2016).

[181] Z. Lu, H. Xu, H. Zeng, and X. Zhang, "Solvent effects on the formation of surface nanodroplets by solvent exchange", Langmuir 31, 12120-12125 (2015).

[182] M. Li, L. Bao, H. Yu, and X. Zhang, "Formation of multicomponent surface nanodroplets by solvent exchange", J. Phys. Chem. C 122, 86478654 (2018).

[183] Y. Wang, H. Wang, S. Bi, and B. Guo, "Automatic morphological characterization of nanobubbles with a novel image segmentation method and its application in the study of nanobubble coalescence", Beilstein J. Nanotechnol. 6, 952-963 (2015).

[184] Y. Wang, X. Li, S. Ren, A. H. Tedros, L. Yang, and D. Lohse, "Entrapment of interfacial nanobubbles on nano-structured surfaces", Soft Matter 13, 5381-5388 (2017).

[185] X. H. Zhang, J. Wang, L. Bao, R. C. A. v. d. V. E. Dietrich, S. Peng, J. Friend, H. J. W. Zandvliet, L. Yeo, and D. Lohse, "Mixed mode of dissolving immersed nanodroplets at a solid-water interface", Soft Matter 11, 1889 (2015). 
[186] Y. M. Song, Y. Xie, V. Malyarchuk, J. Xiao, I. Jung, K.-J. Choi, Z. Liu, H. Park, C. Lu, R.-H. Kim, R. Li, K. B. Crozier, Y. Huang, and J. A. Rogers, "Digital cameras with designs inspired by the arthropod eye", Nature 497, 95-99 (2013).

[187] Goto, K and Kim, YJ and Kirigaya, T and Masuda, Y, "Near-field evanescent wave enhancement with nanometer-sized metal grating and microlens array in parallel optical recording head", Jpn. J. Appl. Phys. 43, 5814-5818 (2004).

[188] H.-R. Jiang, N. Yoshinaga, and M. Sano, "Active motion of a janus particle by self-thermophoresis in a defocused laser beam", Phys. Rev. Lett. 105, 268302 (2010).

[189] Y. Wang, X. Li, S. Bi, X. Zhu, and J. Liu, "3D micro-particle image modeling and its application in measurement resolution investigation for visual sensing based axial localization in an optical microscope", Meas. Sci. Technol. 28 (2017).

[190] Y. Wang, H. Wang, and S. Bi, "Real time drift measurement for colloidal probe atomic force microscope: a visual sensing approach", AIP Adv. 4 (2014).

[191] H. Zhang, L. Xu, Y. Xu, G. Huang, X. Zhao, Y. Lai, and T. Shi, "Enhanced Self-Organized Dewetting of Ultrathin Polymer Blend Film for Large-Area Fabrication of SERS Substrate", Sci. Rep. 6 (2016).

[192] J. Y. Lee, B. H. Hong, W. Y. Kim, S. K. Min, Y. Kim, M. V. Jouravlev, R. Bose, K. S. Kim, I.-C. Hwang, L. J. Kaufman, C. W. Wong, P. Kim, and K. S. Kim, "Near-field focusing and magnification through selfassembled nanoscale spherical lenses", Nature 460, 498-501 (2009).

[193] W. Fan, B. Yan, Z. Wang, and L. Wu, "Three-dimensional all-dielectric metamaterial solid immersion lens for subwavelength imaging at visible frequencies", Sci. Adv. 2, e1600901 (2016).

[194] H. Zhu, M. Chen, S. Zhou, and L. Wu, "Synthesis of high refractive index and shape controllable colloidal polymer microspheres for superresolution imaging", Macromolecules 50, 660-665 (2017).

[195] A. Vlad, I. Huynen, and S. Melinte, "Wavelength-scale lens microscopy via thermal reshaping of colloidal particles", Nanotechnology 23, 285708 (2012). 
[196] A. Verma and A. Sharma, "Enhanced self-organized dewetting of ultrathin polymer films under water-organic solutions: fabrication of submicrometer spherical lens arrays", Adv. Mater. 22, 5306-5309 (2010).

[197] A. Verma, S. Selchar, P. Sachan, P. D. S. Reddy, and A. Sharma, "Control of morphologies and length scales in intensified dewetting of electron beam modified polymer thin films under a liquid solvent mixture", Macromolecules 48, 3318-3326 (2015).

[198] A. Verma and A. Sharma, "Self-organized nano-lens arrays by intensified dewetting of electron beam modified polymer thin-films", Soft Matter 7, 11119-11124 (2011).

[199] A. Verma and A. Sharma, "Submicrometer pattern fabrication by intensification of instability in ultrathin polymer films under a water-solvent mix", Macromolecules 44, 4928-4935 (2011).

[200] R. Mukherjee, D. Bandyopadhyay, and A. Sharma, "Control of morphology in pattern directed dewetting of thin polymer films", Soft Matter 4, 2086-2097 (2008).

[201] X. Lin, A. Hosseini, X. Dou, H. Subbaraman, and R. T. Chen, "Low-cost board-to-board optical interconnects using molded polymer waveguide with 45 degree mirrors and inkjet-printed micro-lenses as proximity vertical coupler", Opt. Express 21, 60-69 (2013).

[202] E. P. Chan and A. J. Crosby, "Fabricating microlens arrays by surface wrinkling", Adv. Mater. 18, 3238-3242 (2006).

[203] J. Shao, Y. Ding, H. Zhai, B. Hu, X. Li, and H. Tian, "Fabrication of large curvature microlens array using confined laser swelling method", Opt. Lett. 38, 3044-3046 (2013).

[204] X. H. Zhang, J. M. Ren, H. J. Yang, Y. H. He, J. F. Tan, and G. G. Qiao, "From transient nanodroplets to permanent nanolenses", Soft Matter 8, 4314-4317 (2012).

[205] Y. Wang, B. Zeng, X. Li, and X. Zhang, "Sequential evaporation-induced formation of polymeric surface microdents via Ouzo effect", Adv. Mater. Interfaces 6, 1900002 (2019). 
[206] B. Zeng, Y. Wang, X. Zhang, and D. Lohse, "Solvent exchange in a hele-shaw cell: Universality of surface nanodroplet nucleation", J. Phys. Chem. C 123, 5571-5577 (2019).

[207] D. Kang, C. Pang, S. M. Kim, H. S. Cho, H. S. Um, Y. W. Choi, and K. Y. Suh, "Shape-controllable microlens arrays via direct transfer of photocurable polymer droplets", Adv. Mater. 24, 1709-1715 (2012).

[208] M. B. G. de Doz, C. M. Bonatti, and H. N. Sólimo, "Liquid-liquid equilibria of ternary and quaternary systems with two hydrocarbons, an alcohol, and water at $303.15 \mathrm{k}$ : Systems containing 2,2,4-trimethylpentane, toluene, methanol, and water, or 2,2,4-trimethylpentane, toluene, ethanol, and water", Fluid Phase Equilib. 205, 53-67 (2003).

[209] E. Lepeltier, C. Bourgaux, and P. Couvreur, "Nanoprecipitation and the "Ouzo effect": Application to drug delivery devices", Adv. Drug Delivery Rev. 71, 86-97 (2014).

[210] L. Pauchard and C. Allain, "Stable and unstable surface evolution during the drying of a polymer solution drop", Phys. Rev. E 68, 052801 (2003).

[211] J. Park and J. Moon, "Control of colloidal particle deposit patterns within picoliter droplets ejected by ink-jet printing", Langmuir 22, 35063513 (2006).

[212] R. Seemann, S. Herminghaus, and K. Jacobs, "Dewetting patterns and molecular forces: A reconciliation", Phys. Rev. Lett. 86, 5534-5537 (2001).

[213] D. S. Fryer, R. D. Peters, E. J. Kim, J. E. Tomaszewski, J. J. De Pablo, P. F. Nealey, C. C. White, and W.-l. Wu, "Dependence of the glass transition temperature of polymer films on interfacial energy and thickness", Macromolecules 34, 5627-5634 (2001).

[214] F. Wang, L. Liu, H. Yu, Y. Wen, P. Yu, Z. Liu, Y. Wang, and W. J. $\mathrm{Li}$, "Scanning superlens microscopy for non-invasive large field-of-view visible light nanoscale imaging", Nat. Commun. 7, 13748 (2016).

[215] A. Darafsheh, N. I. Limberopoulos, J. S. Derov, D. E. Walker, Jr., and V. N. Astratov, "Advantages of microsphere-assisted super-resolution imaging technique over solid immersion lens and confocal microscopies", Appl. Phys. Lett. 104, 061117 (2014). 
[216] D. Beattie, K. Wong, C. Williams, L. Poole-Warren, T. Davis, C. BarnerKowollik, and M. Stenzel, "Honeycomb-structured porous films from polypyrrole-containing block copolymers prepared via RAFT polymerization as a scaffold for cell growth", Biomacromolecules 7, 1072-1082 (2006).

[217] C. Liu, J. Liu, D. Gao, M. Ding, and J.-M. Lin, "Fabrication of microwell arrays based on two-dimensional ordered polystyrene microspheres for high-throughput single-cell analysis", Anal. Chem. 82, 9418-9424 (2010).

[218] F. Chen, S. Mao, H. Zeng, S. Xue, J. Yang, H. Nakajima, J.-M. Lin, and K. Uchiyama, "Inkjet nanoinjection for high-thoughput chemiluminescence immunoassay on multicapillary glass plate", Anal. Chem. 85, 7413-7418 (2013).

[219] Y. Vasquez, M. Kolle, L. Mishchenko, B. D. Hatton, and J. Aizenberg, "Three-phase co-assembly: In situ incorporation of nanoparticles into tunable, highly ordered, porous silica films", ACS Photonics 1, 53-60 (2014).

[220] C. I. Aguirre, E. Reguera, and A. Stein, "Tunable colors in opals and inverse opal photonic crystals", Adv. Funct. Mater. 20, 2565-2578 (2010).

[221] X. Li, Y. Wang, B. Zeng, Y. Li, H. Tan, H. J. Zandvliet, X. Zhang, and D. Lohse, "Entrapment and dissolution of microbubbles inside microwells", Langmuir 34, 10659-10667 (2018).

[222] J. D. Smith, R. Dhiman, S. Anand, E. Reza-Garduno, R. E. Cohen, G. H. McKinley, and K. K. Varanasi, "Droplet mobility on lubricantimpregnated surfaces", Soft Matter 9, 1772-1780 (2013).

[223] A. Lafuma and D. Quere, "Slippery pre-suffused surfaces", Europhys. Lett. 96 (2011).

[224] P. Kim, T.-S. Wong, J. Alvarenga, M. J. Kreder, W. E. AdornoMartinez, and J. Aizenberg, "Liquid-infused nanostructured surfaces with extreme anti-ice and anti-frost performance", ACS Nano 6, 65696577 (2012).

[225] S. Anand, A. T. Paxson, R. Dhiman, J. D. Smith, and K. K. Varanasi, "Enhanced condensation on lubricant-impregnated nanotextured surfaces", ACS Nano 6, 10122-10129 (2012). 
[226] J.-W. Jang, Z. Zheng, O.-S. Lee, W. Shim, G. Zheng, G. C. Schatz, and C. A. Mirkin, "Arrays of nanoscale lenses for subwavelength optical lithography", Nano Lett. 10, 4399-4404 (2010).

[227] R. Pericet-Camara, E. Bonaccurso, and K. Graf, "Microstructuring of polystyrene surfaces with nonsolvent sessile droplets", ChemPhysChem 9, 1738-1746 (2008).

[228] G. Li, K. Graf, E. Bonaccurso, D. S. Golovko, A. Best, and H.-J. Butt, "Evaporation structures of solvent drops evaporating from polymer surfaces: Influence of molar mass", Macromol. Chem. Phys. 208, 2134-2144 (2007).

[229] F. Khan, R. Zhang, A. Unciti-Broceta, J. J. Diaz-Mochon, and M. Bradley, "Flexible fabrication of microarrays of microwells", Adv. Mater. 19, 3524-3528 (2007).

[230] G. Li and K. Graf, "Microstructures formation by deposition of toluene drops on polystyrene surface", Phys. Chem. Chem. Phys. 11, 7137-7144 (2009).

[231] J. Zhang, Y. Li, X. Zhang, and B. Yang, "Colloidal self-assembly meets nanofabrication: From two-dimensional colloidal crystals to nanostructure arrays", Adv. Mater. 22, 4249-4269 (2010).

[232] A. S. de Leon, A. del Campo, C. Labrugere, M. Fernandez-Garcia, A. Munoz-Bonilla, and J. Rodriguez-Hernandez, "Control of the chemistry outside the pores in honeycomb patterned films", Polym. Chem. 4, 4024-4032 (2013).

[233] A. S. de Leon, A. del Campo, M. Fernandez-Garcia, J. RodriguezHernandez, and A. Munoz-Bonilla, "Hierarchically structured multifunctional porous interfaces through water templated self-assembly of ternary systems", Langmuir 28, 9778-9787 (2012).

[234] P. A. Gurr, Z. Zhang, X. Hao, T. C. Hughes, and G. G. Qiao, "Highly ordered honeycomb film formation of linear polymers by the breath figure technique", Aust. J. Chem. 69, 1130-1139 (2016).

[235] P. Jiang, "Large-scale fabrication of periodic nanostructured materials by using hexagonal non-close-packed colloidal crystals as templates", Langmuir 22, 3955-3958 (2006). 
[236] M. Abdelsalam, P. Bartlett, J. Baumberg, and S. Coyle, "Preparation of arrays of isolated spherical cavities by self-assembly of polystyrene spheres on self-assembled pre-patterned macroporous films", Adv. Mater. 16, 90-93 (2004).

[237] Z. Lu, M. H. K. Schaarsberg, X. Zhu, L. Y. Yeo, D. Lohse, and X. Zhang, "Universal nanodroplet branches from confining the Ouzo effect", Proc. Natl. Acad. Sci. U. S. A. 114, 10332-10337 (2017).

[238] E. Aschenbrenner, K. Bley, K. Koynov, M. Makowski, M. Kappl, K. Landfester, and C. K. Weiss, "Using the polymeric Ouzo effect for the preparation of polysaccharide-based nanoparticles", Langmuir 29, 8845-8855 (2013).

[239] H. Tan, C. Diddens, M. Versluis, H.-J. Butt, D. Lohse, and X. Zhang, "Self-wrapping of an ouzo drop induced by evaporation on a superamphiphobic surface", Soft Matter 13, 2749-2759 (2017).

[240] J. H. Walton and J. D. Jenkins, "A study of the ternary system, tolueneacetone-water", J. Am. Chem. Soc. 45, 2555-2559 (1923).

[241] I. Smallwood, Handbook of organic solvent properties (ButterworthHeinemann) (2012).

[242] K. Liu, M. Cao, A. Fujishima, and L. Jiang, "Bio-inspired titanium dioxide materials with special wettability and their applications", Chem. Rev. 114, 10044-10094 (2014).

[243] S. A. Kulinich and M. Farzaneh, "Effect of contact angle hysteresis on water droplet evaporation from super-hydrophobic surfaces", Appl. Surf. Sci. 255, 4056-4060 (2009). 


\section{Summary}

There are plenty of natural and industrial processes involving bubbles and droplets. It is essential to understand the nucleation mechanism and dynamics of the bubbles/droplets for achieving controllable production of bubbles/droplets in relevant industrial applications. This thesis studies two parts: (I) Plasmonic bubbles in binary liquids. We experimentally and theoretically studied the nucleation and growth dynamics of the plasmonic bubbles in binary liquids and under different ambient pressures. (II) Droplets from the Ouzo effect. We investigated the droplet nucleation through the Ouzo effect in a well-controlled Hele-Shaw cell, and proposed feasible methods to produce microlenses and microdents based on the Ouzo effect.

In part I, we first studied the nucleation (short term) of the initial giant plasmonic bubble in water (Chapter 2). In this work, the ambient pressure was varied from 1 bar to 9 bar by a pressure chamber, and the evolution of the initial giant bubbles was captured by high-speed cameras. We revealed that the delay time between the start of laser heating and the bubble nucleation, and bubble nucleation temperature are governed by the absolute gas concentration in the water. Furthermore, it is demonstrated that the light vapour conversion efficiency strongly decreases with increasing ambient pressures, due to the increased boiling point of water with increasing ambient pressures.

We further studied the nucleation (short term, Chapter 3), bouncing behaviour (mid term, Chapter 4) and shrinkage event (long term, Chapter 5) of the plasmonic bubbles in binary liquids. In Chapter 3, we focused on the initial giant plasmonic bubble nucleation for six binary liquids and measured the delay time $\tau_{d}$ of bubble nucleation after turning on the laser and the maximum size of the bubbles. The experimental results showed that the delay time $\tau_{d}$ depends on the absolute amount of dissolved gas in the liquid. Moreover, we revealed that at a given dumped energy prior to the formation of the bubble, the energy of the liquid required for vaporization governs the maximum size of the giant bubble. In Chapter 4 , we found a periodically bouncing behaviour of 
a bubble within tens of milliseconds after the initial giant bubble nucleation. We experimentally and theoretically studied this bouncing behaviour and revealed that it originates from the competition between thermal and solutal Marangoni forces acting on the bubble. A theoretical model was proposed to qualitatively describe this process. The theoretical bouncing frequencies agrees well with the experimental observations. In Chapter 5, we investigated the long term behaviour of the plasmonic bubbles in binary liquids. The underlying mechanism of a sudden shrinkage during bubble growth with an ethanol ratio $70 \%-80 \%$ was revealed. The sudden shrinkage is caused by a depinning of the three-phase contact line from the laser spot region.

In part II, we experimentally investigated the formation of surface droplets through the Ouzo effect (Chapter 6). We have applied the droplets in the mass production of microlenses (Chapter 7) and microdents (Chapter 8) based on the Ouzo effect. In Chapter 6, a Hele-Shaw cell was used to study the droplet formation through the Ouzo effect. We observed that the obtained droplets are more homogeneous, and the mean droplet volume per area $\langle V o l\rangle_{\text {area }}$ depends on the local $P e$ number, following the universal scaling law $\langle V o l\rangle_{\text {area }} \sim P e^{3 / 4}$. Furthermore, we found that the droplet volume's probability distribution function is governed by local $\mathrm{Pe}$ as well. In Chapter 7, we took advantage of the controllable formation of micro/nanodroplets from the Ouzo effect and designed a simple method to obtain microlenses. In this method, we added water to the toluene-ethanol binary liquids to trigger the Ouzo effect. The toluene droplets separated from the ethanol and dissolved the PS film to generate toluene-PS binary droplets on the substrate. Eventually, the toluene in toluene-PS droplets dissolved into the bulk again, and we obtained PS microlenses on the substrate. In Chapter 8, we applied the droplets obtained using the Ouzo effect for the fabrication of micro indents. In this study, we placed an acetone-toluene-water ternary liquid with dissolved PS on a glass substrate. The Ouzo effect was induced after acetone was evaporated, resulting in the nucleation of water droplets, which act as the template. After that, the toluene evaporated, and the PS precipitated around the water droplets. Finally, we obtained PS micro indents after the water droplets evaporated. 


\section{Summary (Dutch)}

Er zijn tal van natuurlijke en industriële processen waarin bellen en druppels een belangrijke rol spelen. Het is essentieel om het nucleatiemechanisme en de dynamica van de bellen/druppels te begrijpen om een controleerbare productie van bellen/druppels in relevante industriële toepassingen mogelijk te maken. Dit proefschrift bestaat uit twee delen: (I) Plasmonische bellen in binaire vloeistoffen. We bestudeerden zowel experimenteel als theoretisch de nucleatie en groei van de plasmonische bellen in binaire vloeistoffen bij verschillende omgevingsdrukken. (II) Druppels die optreden in het Ouzo-effect. We onderzochten de druppelkernen gebruikmakend van het Ouzo-effect in een goed gecontroleerde Hele-Shaw cel. We hebben methoden voorgesteld voor de productie van microlenzen en microdeeltjes op basis van het Ouzo-effect.

In deel I bestudeerden we eerst de nucleatie (op korte termijn) van de initiële plasmonische reuzenbel in water (hoofdstuk 2). In dit werk werd de omgevingsdruk gevarieerd van 1 bar tot 9 bar. De evolutie van de initiële reuzenbellen werd vastgelegd door hogesnelheidscamera's. We toonden aan dat de vertragingstijd tussen het begin van de laserverwarming en de kiemvorming van de bel alsmede de kiemvormingstemperatuur van de bel bepaald worden door de absolute gasconcentratie in het water. Verder is aangetoond dat de lichtdampconversie-efficiëntie sterk afneemt met toenemende omgevingsdruk, als gevolg van het verhoogde kookpunt van water met toenemende omgevingsdruk.

We bestudeerden verder de nucleatie (korte termijn, hoofdstuk 3), het stuitergedrag (middellange termijn, hoofdstuk 4 ) en de krimp (lange termijn, hoofdstuk 5) van plasmonische bellen in binaire vloeistoffen. In hoofdstuk 3 richtten we ons op de initiële plasmonische kiemvorming van de bel voor zes binaire vloeistoffen. We hebben de vertragingstijd $\tau_{d}$ van de kiemvorming van de bel na het inschakelen van de laser en de maximale grootte van de bellen bepaald. De experimentele resultaten toonden aan dat de vertragingstijd $\tau_{d}$ 
afhankelijk is van de absolute hoeveelheid opgelost gas in de vloeistof. Bovendien toonden we aan dat bij een gegeven afgestane hoeveelheid energie voorafgaand aan de vorming van de bel, de energie die nodig is voor de verdamping van de vloeistof de maximale grootte van de reuzenbel bepaald. In hoofdstuk 4 vonden we een periodiek stuiterend gedrag van een bel binnen tientallen milliseconden na de initiële vorming van een reuzenbel. We bestudeerden dit stuitergedrag experimenteel en theoretisch en ontdekten dat het voortkomt uit de competitie tussen thermische en solutaire Marangoni krachten die op de bel werken. Een theoretisch model werd voorgesteld om dit proces kwalitatief te beschrijven. De theoretische stuiterfrequenties komen goed overeen met de experimentele waarnemingen. In hoofdstuk 5 hebben we het lange termijn gedrag van plasmonische bellen in binaire vloeistoffen onderzocht. Het onderliggende mechanisme van een plotselinge krimp tijdens de groei van bellen bij een ethanolverhouding van 70-80\% werd ontrafeld. Het plotselinge krimpen wordt veroorzaakt door een depinning van de driefasige contactlijn van de laserspot.

In deel II hebben we experimenteel de vorming van oppervlaktedruppels door het Ouzo effect onderzocht (hoofdstuk 6). We hebben de druppels toegepast in de massaproductie van microlenzen (hoofdstuk 7) en microdeukjes (hoofdstuk 8) op basis van het Ouzo-effect. In hoofdstuk 6 werd een HeleShaw cel gebruikt om de druppelvorming door het Ouzo-effect te bestuderen. We stelden vast dat de verkregen druppels meer homogeen zijn, en dat het gemiddelde druppelvolume per oppervlakte $\langle V o l\rangle_{\text {area }}$ afhangt van het lokale $P e$ getal, volgens de universele schalingswet $\langle V o l\rangle_{\text {area }} \sim P e^{3 / 4}$. Bovendien vonden we dat de kansverdelingsfunctie van het druppelvolume ook bepaald wordt door het lokale $P e$ getal. In hoofdstuk 7 hebben we gebruik gemaakt van de controleerbare vorming van micro/nanodruppels die optreden in het Ouzo-effect en hebben we een eenvoudige methode ontwikkeld om microlenzen te realiseren. In deze methode voegden we water toe aan een tolueen-ethanol binaire vloeistof om het Ouzo-effect op te wekken. De tolueen druppels scheidden van de ethanol en losten de polystyreen (PS) film op waarna vervolgens tolueen-PS binaire druppels op het substraat te ontstonden. Uiteindelijk loste de tolueen in de tolueen-PS druppels weer op in de bulk, en verkregen we PS microlenzen op het substraat. In hoofdstuk 8 hebben we de druppels verkregen met behulp van het Ouzo effect toegepast voor de fabricage van microdeukjes. In deze studie plaatsten we een aceton-tolueen-water ternaire vloeistof met opgelost PS op een glazen substraat. Het Ouzo-effect werd geïnduceerd nadat aceton was verdampt, wat resulteerde in de nucleatie van waterdruppels, die 
fungeren als sjabloon. Daarna verdampte de tolueen, en het PS sloeg neer rond de waterdruppels. Tenslotte verkregen we PS microdeukjes nadat de waterdruppels verdampt waren. 


\section{Acknowledgements}

Three years have passed since I started my journey to the Physics of Fluid group and in January 2019. I would like to show my great gratitude to many people for your interaction and supports.

Dear Detlef, thank you for accepting me to be a joint Ph.D. student in PoF. I still remembered that my first talk with you was on my droplet project in a restaurant in Beijing in 2018. I was very nervous at that time because it was my first time to present my work in front of a knowledgeable scientist, while your passion and concentration guide me into scientific problems soon and lead me in an relaxed atmosphere. During my study at PoF, you guided me into the field of fluid physics, provided massive supports in my project, encouraged me to overcome difficulties in experiments, and taught me how to be a scientific researcher. I truly admired your enthusiasm, passion and sharp sense of science, precise writing and concise presentation skills, diligence, humor towards people, and your caring for students. I really appreciate being your student, and will always follow the example of you on the road ahead!

Dear Harold, thank you for your continuous support during my $\mathrm{PhD}$ study. Our first meet was in Nov. 2018 during your visit to Beijing. I am impressed with your kindness and enthusiasm and enjoyed the conversation with you very much. I felt very excited with the coming journey to Netherlands. After my arrival, it is lucky to have you as my supervisor. Whenever I looked for help, you are always available. You not only consider whether my project going smoothly or need support, but also remind me to live a happy and healthy life. I really like the nice environment in Netherlands and friendly people here. During my stay in UT, I learned a lot from your numerous suggestions and improved a lot. I am grateful for your help in the arrangements for my $\mathrm{PhD}$ study. I will never forget this beautiful memory in the Netherlands!

Dear Yuliang, I appreciate that you accept me as your Ph.D. student in 2016. At that time, I was hesitating to continue to study Ph.D. or enter the industrial community directly after master graduation. Now, five years passed, 
I would say that joining your group for Ph.D. study is an absolute correct decision. During the past years, I learned so much from you and improved a lot. In the beginning I was unfamiliar with fundamental research because I mainly worked on engineering for master study. You not only taught me to do experiment personally, but are always patient to guide and encourage me during our discussion. I am an introvert and unconfident person. You provide me many training opportunities, like organizing group meetings, attending AFM operation training, and promoting me to study abroad. I truly appreciate all your support and help.

Dear Xuehua, thank you for your valuable supports of my research. Our first conversion was in 2018 by Wechat. At that time, I was struggling with the work of the microlenses. Your comments and encouragements promoted me to overcome the difficulties. Finally, we made it. At the end of 2019, it was our first face-to-face meeting when you visited PoF in the Netherlands. I admired your knowledge of droplets \& bubbles, full of passion and kindness. I appreciated the fruitful interaction with you.

Dear Andrea, many thanks for your fruitful discussion in Jan. 2020. I really admire your enthusiasm for physics.

Dear Corinna, thank you for your helpful discussion in Feb. 2020. Your sweet works on active droplets driven by the Marangoni effect inspire me a lot.

Dear Joanita, thanks a lot for your support. You can always resolve problems efficiently whenever I asking for help such as applying for visa and residence permit, finding houses, arrange meetings, conferences and defences, etc. Without your help I could not well concentrate on my research as now.

Dear Dennis, thank you for your dedication to managing all labs and all your friendless. Dear Bas and Thomas, thanks for ordering materials and arranging a computer for me. Dear Martin and Gert-Wim, thanks a lot for your supports on the experimental setup which helps me work more efficiently.

\section{Main collaborators:}

Mikhail, thanks a lot for teaching me to using the setup of plasmonic bubble. You are always very kind and generous when I ask for help.

Marvin, you are always full of passion and ideas, thanks a lot for your discussions and comments in our group meeting. I also appreciate the corporation and playing sports with you. Wish you a good luck in your future.

Hai, thank you for providing us very nice and robust samples.

Luoqin, I am very grateful to you for your numerous help. I heard a lot 
about your outstanding ability in theory of physic before my coming to PoF, but I was still surprised when I had opportunities to discuss with you. You have very sharp physical insight and knows everything on my work quickly though you just learned it from my brief description. I truly appreciate your helpful suggestions on my bouncing and dissolution projects.

Steven, thanks you for your huge support in our joint bouncing bubble project. You are a good teacher of me. You are always patient to answer my theoretical questions and explain everything clearly to me. Sometimes you keep discussing with me on my project till 1-2 am in the midnight. I learned a lot about theoretical modelling from you, and improve physical insight from your fruitful discussions. I am very grateful on all your great efforts and help.

Yanshen, many thanks to you for your continuous support in my work and life. You are like a brother to me. Whenever I have questions or difficulties, you always tried your best to help me to solve them. You also taught me to use Contact Angle Meter and many devices in biolab, and provide me any reagents/materials whenever I need. I like discussing scientific problems with you, and admire your outstanding intelligence, and critical/precise scientific spirit very much. Your diligence and concentration in work always inspire me to work harder and think deeper.

Christian, I appreciate your support in the numerical work. You are knowledgeable in physics and chemistry and full of experience in numerics. I benefit a lot from every communication with you.

I would like to thanks my lab mates.

Ricardo, Srinath and Lijun, I feel lucky to have such kind and responsible labmates like you. Thanks a lot for your interaction, and good luck with your future. Srinath, I also really enjoy the time to play badminton with you.

My special thanks to:

Shuai, thanks a lot for the helpful discussions on cavitation bubble. I learned a lot from the several nice conversations.

Haoran, thank you for the fruitful discussions on the surface energy. Also thanks for your friendiness to me. I really enjoy the spicy duck necks in your home.

Sarah, thanks a lot for the helpful discussions on PIV analysis. I also appreciate your hard working and kindness.

Guiquan, I really enjoy the conversion with you in the office. Your optimism and enthusiasm create a happy atmosphere around us. 
Jiaming, thank you for answering my questions in looking for jobs. I am grateful to all your encouragement and support.

Shuang, thank you for your accompany and support the first time when I study at PoF. It is a beautiful memory to study and play with you.

Nayoung, I enjoy the nice time with you. You are very friendly and considerate to us. Thank you for your nice Korea foods.

Pallav, thanks for your helping in labs and many others. I also admire your gentleness and dedication.

Yaxing, thank you for your helping around in labs and many others. You are always very considerate and friendly to everyone of us. I also appreciate your optimistic and positive spirit.

Yogesh, thank you for teaching me how to use the pulse laser. You are always very polite to me, and I also appreciate your hard-working.

Qi, I really enjoy the time when I played poker and Werewolf with you. You are a God of Gamblers and performer. I also respect your dedicated spirit and hard-working.

Yibo, thank you for explaining your simulation work on droplet instability, and I learned how to run numerics from you. You are always enthusiastic for life, and ready to help others. I am glad that we work together on Qi's Movie. You were a good director, and we finally made a hilarious video.

Rui, thank you very much for your careful response whenever I was asking you theoretical problems. You are a very professional cameraman, and I really enjoy every pictures you took for us.

Ellis, thanks a lot for the delicious Malaysian foods for us. I also admire your perfessional badminton skill.

Yoan, I really enjoy the nice conversations with you. Thanks for introducing me interesting websites about historical story.

Çayan, thanks a lot for your kindness to us. I admire your smiling and positive attitude towards life.

Peter (Confucius) and Timothy, thank you for your interactions. I feel very interesting in communicating with you in Cantonese, and thank you for tolerating my broken Cantonese.

Hanif, Saeed, Morgan and Jessica, I enjoy the time we playing badminton together.

Nathan, Yelle, Minkush, Vanshu, Diana, Vatsal, Hadi, Nakul, Ryo, Srinidhi, Jessica, Dawid and Youssef, you are always very friendly and kind to me. I enjoyed the nice conversations with you.

I would extend my great thanks to all PoFers, PINers: Tim, Ambre, 
Pierre, Charu, Ariane, Tuong, Chris, Utkarsh, CS, Pablo, Udo, Mathieu, Martin, Simon, Luuk, Benjamin, Stefan, Martin, Daniel, Michiel, Robert, Majorie, Naoki, Wojtek, Jochem, Jeffrey, Charlotte, Edgar, Bernardo, Giulia, Prashanth, Ali, Maaike, Farzan, Carola, Anja, Lennart, Zhiguo, ..... Forgive me if I foget someone due to my bad memory. Thank you for creating a nice working environment.

Finally, I owe my thanks to my parents, my brother, sisters and Xiaolai. 爸爸妈妈, 感谢你们的养育之恩和一直以为对我不求回报的支持。

岳父岳母, 感谢你们能够培养出如此优秀的女儿, 以及你们一直以来对我 的包容。

哥哥, 感谢你一直以来对爸爸妈妈的陪伴, 以及对我无限的支持。 大姐二姐以及姐夫，感谢你们一直以来对我和晓来的照顾和支持。

晓来, 今年是我们在一起的第十一年, 非常庆幸我们能够在人生最美好的 时候相遇, 相知到相爱。我们一起经历风雨, 一起成长。感谢有你的每一个日 夜, 愿执子之手, 与子偕老! 


\section{Scientific output}

\section{Publications}

- 2021:

Binglin Zeng, Kai Leong Chong, Yuliang Wang, Christian Diddens, Xiaolai Lia, Marvin Detert, Harold J. W. Zandvliet, and Detlef Lohse Periodic bouncing of a plasmonic bubble in a binary liquid by competing solutal and thermal Marangoni forces

Proc. Natl. Acad. Sci. U. S. A. 118, e2103215118 (2021) - see Chapter 4

Binglin Zeng, Christian Diddens, Yuliang Wang, Harold J. W. Zandvliet, and Detlef Lohse

Droplet dissolution driven by Marangoni flow

In preparation.

- 2020:

Binglin Zeng, Yuliang Wang, Mikhail E. Zaytsev, Chenliang Xia, Harold J. W. Zandvliet, and Detlef Lohse

Giant plasmonic bubbles nucleation under different ambient pressures

Phys. Rev. E 102, 063109 (2020) - see Chapter 2

Marvin Detert, Binglin Zeng, Yuliang Wang, Hai Le The, Harold J. W. Zandvliet, and Detlef Lohse

Plasmonic bubble nucleation in binary liquids

J. Phys. Chem. C 124, 2591-2597 (2020) - see Chapter 3

Xiaolai Li, Yuliang Wang, Binglin Zeng, Marvin Detert, Andrea Prosperetti, Harold J. W. Zandvliet, and Detlef Lohse Plasmonic microbubble dynamics in binary liquids J. Phys. Chem. Lett. 11, 8631-8637 (2020) - see Chapter 5 
- 2019:

Binglin Zeng, Yuliang Wang, Xuehua Zhang, and Detlef Lohse Solvent exchange in a Hele-Shaw cell: universality of surface nanodroplet nucleation

J. Phys. Chem. C 123, 5571-5577 (2019) - see Chapter 6

Yuliang Wang, Binglin Zeng, Yong Zhao, Shuai Li, and Xuehua Zhang Formation of polystyrene microlenses via transient droplets from the ouzo effect for enhanced optical imaging

J. Phys. Chem. C 123, 14327-14337 (2019) - see Chapter 7]

Yuliang Wang, Binglin Zeng, Xiaolai Li, and Xuehua Zhang

Sequential evaporation induced formation of polymeric surface microdents via ouzo effect

Adv. Mater. Interfaces 6, 1900002 (2019) - see Chapter 8

Dayong Li, Binglin Zeng, Yuliang Wang

Probing the "Gas Tunnel" between Neighboring Nanobubbles

Langmuir 35, 15029-15037 (2019)

\section{- 2018:}

Yuliang Wang, Binglin Zeng, Hadush Tedros Alem, Zaicheng Zhang, Elisabeth Charlaix, and Abdelhamid Maali

Viscocapillary response of gas bubbles probed by thermal noise atomic force measurement

Langmuir, 34, 1371-1375, (2018) 